\title{
Effects of Load and Gradient on Musculoskeletal Loading During Dynamic Two-Wheeled Cart Pushing and Pulling
}

\author{
Yun Sun \\ West Virginia University
}

Follow this and additional works at: https://researchrepository.wvu.edu/etd

\section{Recommended Citation}

Sun, Yun, "Effects of Load and Gradient on Musculoskeletal Loading During Dynamic Two-Wheeled Cart Pushing and Pulling" (2011). Graduate Theses, Dissertations, and Problem Reports. 4801.

https://researchrepository.wvu.edu/etd/4801

This Dissertation is protected by copyright and/or related rights. It has been brought to you by the The Research Repository @ WVU with permission from the rights-holder(s). You are free to use this Dissertation in any way that is permitted by the copyright and related rights legislation that applies to your use. For other uses you must obtain permission from the rights-holder(s) directly, unless additional rights are indicated by a Creative Commons license in the record and/ or on the work itself. This Dissertation has been accepted for inclusion in WVU Graduate Theses, Dissertations, and Problem Reports collection by an authorized administrator of The Research Repository @ WVU.

For more information, please contact researchrepository@mail.wvu.edu. 


\title{
Effects of Load and Gradient on Musculoskeletal Loading During Dynamic Two-Wheeled Cart Pushing and Pulling
}

\author{
Yun Sun \\ Dissertation submitted to the \\ College of Engineering and Mineral Resources \\ at West Virginia University \\ in partial fulfillment of the requirements \\ for the degree of \\ Doctor of Philosophy \\ in \\ Occupational Safety and Health
}

Ashish D. Nimbarte, Ph.D., Chair

Majid Jaraiedi, Ph.D.

Michael J. Klishis, Ph.D.

Avinash Unnikrishnan, Ph.D.

Hongwei Hsiao, Ph.D.

Department of Industrial and Management Systems Engineering

Morgantown, West Virginia

2011

Keywords: Dynamic Pushing and Pulling; Musculoskeletal Loading;

Biomechanical Modeling; Inclined Walkway; Direction of Force Application 


\title{
ABSTRACT \\ Effects of Load and Gradient on Musculoskeletal Loading During Dynamic Two-Wheeled Cart Pushing and Pulling
}

\begin{abstract}
Yun Sun
Pushing and pulling of carts are found to be associated with low back pain and shoulder complaints. It is currently unclear as to how the dynamic pushing and pulling tasks performed under different task conditions (e.g. weight, walkway gradient, direction of force application) affect the musculoskeletal loading of low back and shoulder joints. Therefore, the objective in this study was to evaluate dynamic pushing and pulling tasks to quantify their effects on the 3D musculoskeletal loading of low back and shoulder joints. Twelve male participants performed cart pushing pulling tasks along a $5^{\circ}$ and $10^{\circ}$ ramp by carrying loads of 20,30 , and $40 \mathrm{~kg}$, respectively. An eight-camera optical motion analysis system configured with two force plates was used for kinematic and kinetic data collection. The experimental data was modeled using Anybody musculoskeletal modeling system. A biomechanical model consisting of a two wheeled cart and a full-body human model (34 bones and over 750 muscle fascicles) was formulated in the AnyBody modeling system. The model was validated by comparing the predicted reaction forces on the cart wheels with the actual forces recorded experimentally using ground reaction force platforms. A high correlation between the actual and predicted data (average $=0.919$ ) was observed. The results of this study show that as the cart weight increased, so did the low back and shoulder joint loads. A walkway gradient of $10^{\circ}$ on average produced larger low back and shoulder joint forces than a gradient of $5^{\circ}$. Uphill pushing caused higher mechanical load on the low back and shoulder joints than the downhill pulling. The magnitude of peak lateral shear forces at the L5S1 joint was negligible $(<150 \mathrm{~N})$. The largest peak compression force observed in this study $(3222 \mathrm{~N})$ was below the recommended NIOSH limit of $3400 \mathrm{~N}$. The peak anterior-posterior shear forces during uphill pushing at $30 \mathrm{~kg}$ and $40 \mathrm{~kg}$ weight conditions on a gradient of $10^{\circ}$ were $553 \mathrm{~N}$ and $806 \mathrm{~N}$, respectively. These values exceed the tolerance limit of $500 \mathrm{~N}$, suggesting that the anterior-posterior shear loading at the L5S1 joint should be considered when assessing the risk of musculoskeletal disorders due to pushing and pulling activities.
\end{abstract}

For the shoulder joints, the peak forces at the sternoclavicular joint were small $(<220 \mathrm{~N})$ in all situations. At the glenohumeral joint, the peak distraction, compression and anterior-posterior shear forces were $1357 \mathrm{~N}, 858 \mathrm{~N}$, and $618 \mathrm{~N}$, respectively, during uphill pushing at $40 \mathrm{~kg}$ weight condition on a gradient of $10^{\circ}$. Under the same condition, the corresponding peak forces acting at the acromioclavicular joint were $910 \mathrm{~N}, 1078 \mathrm{~N}$, and $727 \mathrm{~N}$. In the existing literature, the focus has always been on the glenohumeral joint and loading of the sternoclavicular and acromioclavicular joints was not evaluated. The results of this research show that the peak forces acted at the acromioclavicular joint are comparable to those at the glenohumeral joint, further suggesting that the loading on the acromioclavicular joint should be considered, in addition to the glenohumeral joint, while evaluating risk of musculoskeletal disorders due to pushing and pulling activities. 


\section{ACKNOWLEDGEMENTS}

I would like to express my deepest gratitude to my advisor, Dr. Ashish Nimbarte, for his continuous encouragement, guidance, and support during this research. I am deeply indebted to him for his invaluable help in my difficult time.

I would also like to thank the members of my committee, Dr. Majid Jaraiedi, Dr. Michael Klishis, Dr. Avinash Unnikrishnan, and Dr. Hongwei Hsiao for their invaluable comments, suggestions, and insights.

I also wish to acknowledge my doctoral program coordinator, Dr. Gary Winn, who has helped me a lot during my long journey in the program of occupational safety and health. I would also like to thank the department chair, Dr. Wafik Iskander, for his generous financial support for my teaching assistantship.

I would also like to thank my colleagues in the Ergonomics Laboratory, in particular Christopher Moore, for his assistance in the experimental setup part of this study. Many thanks to all the people I have known at West Virginia University, whose friendship I will always treasure.

Finally, I want to express my gratitude to my beloved parents, elder brother, sister-in-law and two lovely nieces, for their patience, support, and understanding during this difficult period of time. 


\section{TABLE OF CONTENTS}

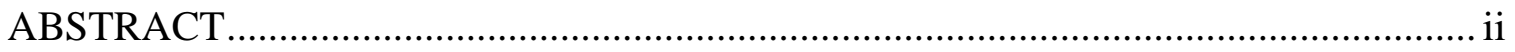

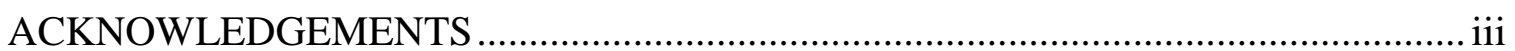

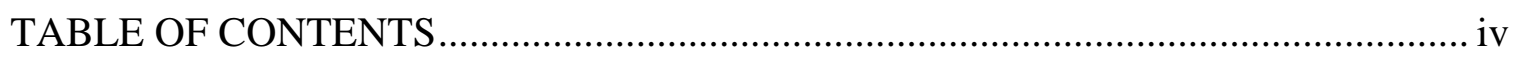

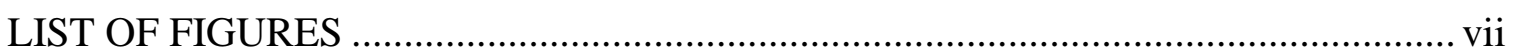

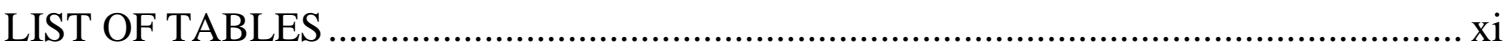

CHAPTER 1: INTRODUCTION ........................................................................ 1

CHAPTER 2: LITERATURE REVIEW …............................................................. 5

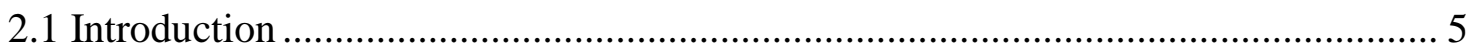

2.2 Work-Related Pushing and Pulling Exertions...................................................... 5

2.3 Musculoskeletal Disorders: Pushing and Pulling .............................................. 6

2.4 Static and Dynamic Exertions ....................................................................... 8

2.5 Factors Affecting Pushing and Pulling......................................................... 10

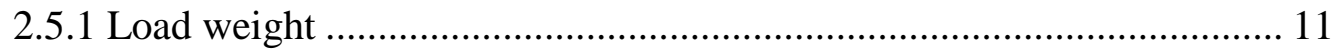

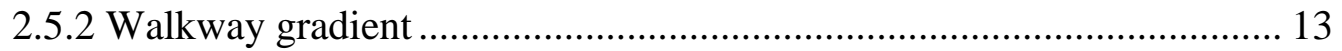

2.5.3 Direction of force application ......................................................... 15

2.6 Musculoskeletal Models for Pushing and Pulling ............................................. 16

2.6.1 Single equivalent muscle models .................................................. 16

2.6.2 EMG-assisted multiple muscle system models ................................. 20

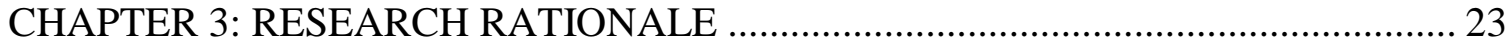

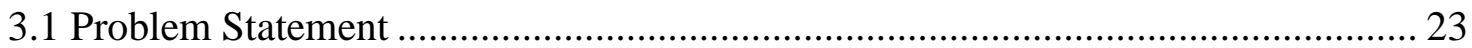

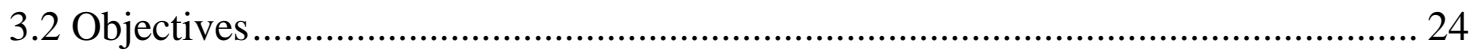

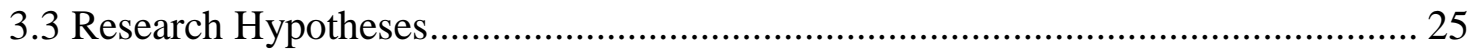

3.3.1 Hypothesis 1: Impact of load weight .................................................. 25

3.3.2 Hypothesis 2: Impact of walkway gradient ..................................... 25

3.3.3 Hypothesis 3: Impact of direction of force application along the ramp . 26

3.3.4 Hypothesis 4: Low back loading in anterior-posterior direction ........... 26

3.3.5 Hypothesis 5: Shoulder loading at acromioclavicular joint ................... 26

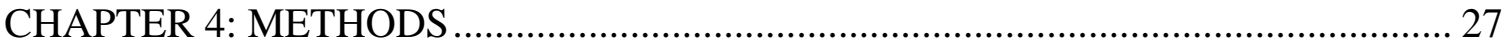

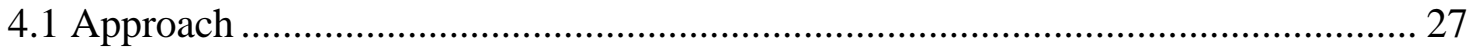

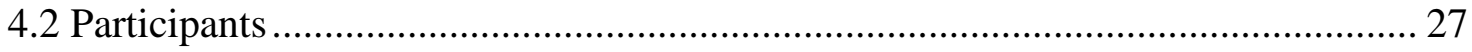




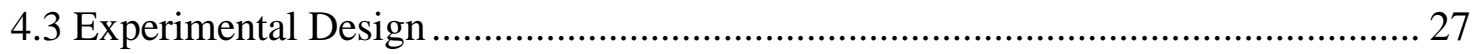

4.3.1 Independent variables ....................................................................... 28

4.3.2 Dependent variables......................................................................... 28

4.3.3 Statistical models .............................................................................. 29

4.3.4 Power analysis and sample size determination ....................................... 33

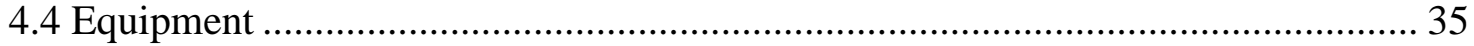

4.4.1 Hand truck and loads ....................................................................... 35

4.4.2 Wooden walkway, scissor lift table and bottle jacks .............................. 36

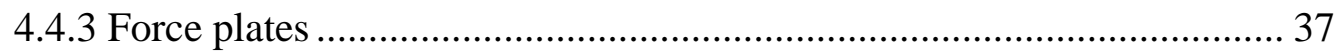

4.4.4 Optical motion capture system ……………………………………...... 39

4.4.5 AnyBody modeling system..................................................................... 42

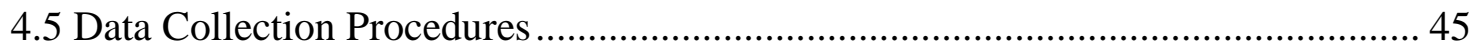

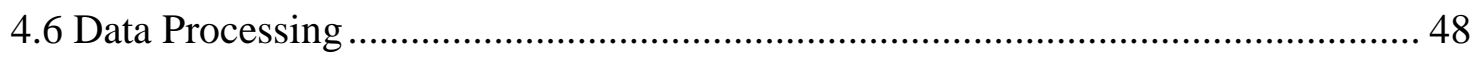

4.6.1 Kinematic data: Vicon Nexus software ……………............................. 48

4.6.2 Musculoskeletal model simulation ........................................................ 51

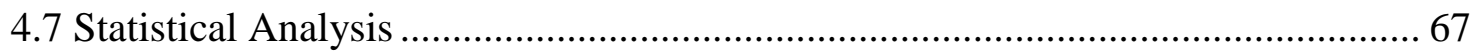

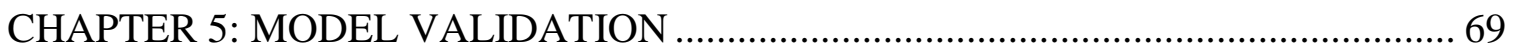

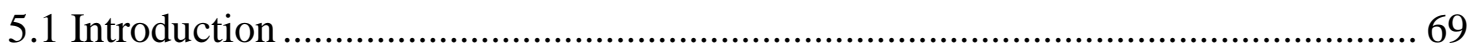

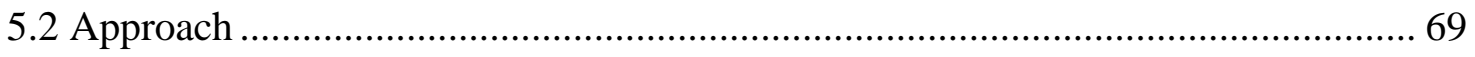

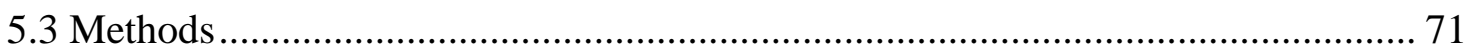

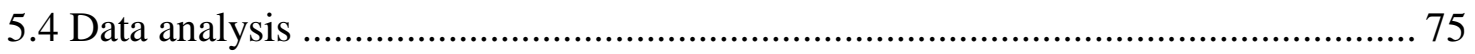

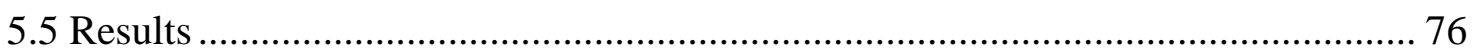

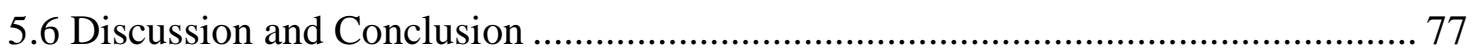

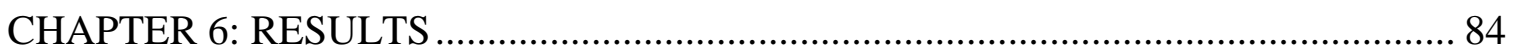

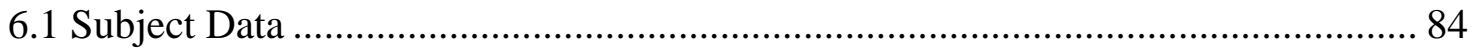

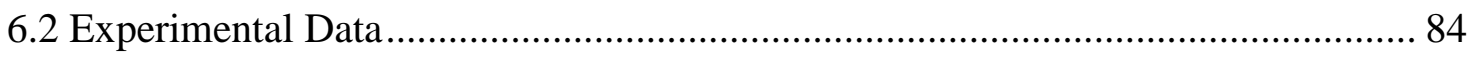

6.2.1 Participants' ground reaction force (GRF) data ..................................... 84

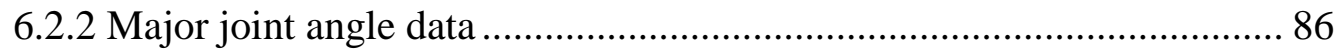

6.3 Model 1: Cart Pushing along a Flat Walkway ........................................................... 86

6.3.1 Low back joint loading at L5S1 ........................................................... 86

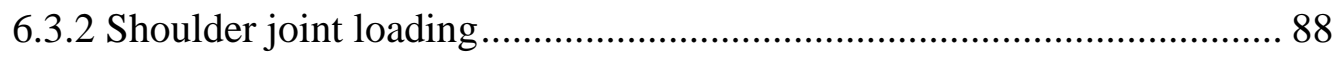

6.4 Model 2: Cart Pushing and Pulling along Ramps .................................................... 91

6.4.1 Low back joint loading at L5S1 ............................................................ 91 
6.4.2 Shoulder joint loading at sternoclavicular joint 99

6.4.3 Shoulder joint loading at acromioclavicular joint. 107

6.4.4 Shoulder joint loading at glenohumeral joint. 114

CHAPTER 7: DISCUSSION 121

7.1 Low Back Loading 121

7.1.1 Effect of load weight. 121

7.1.2 Effect of walkway gradient 122

7.1.3 Effect of direction of force application ........................................ 123

7.1.4 Risk of low back injury .............................................................. 124

7.2 Shoulder Loading 125

7.2.1 Effects of load, gradient, and direction of force application............... 125

7.2.2 Comparison among shoulder complex joints.................................. 126

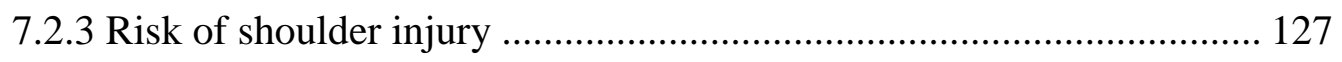

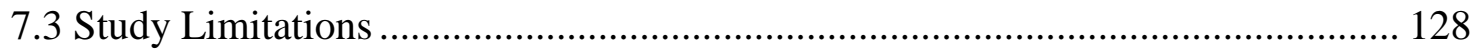

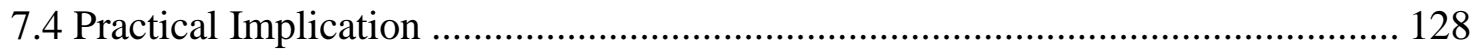

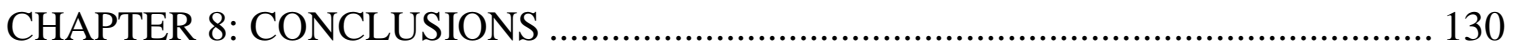

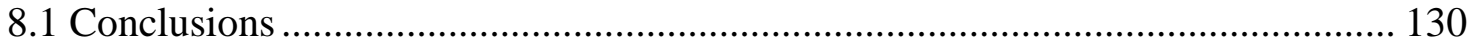

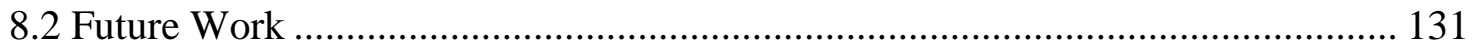

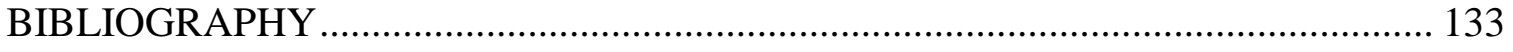

APPENDIX A: PHYSICAL ACTIVITY READINESS QUESTIONNAIRE ............... 146

APPENDIX B: CONSENT AND INFORMATION FORM ...................................... 148

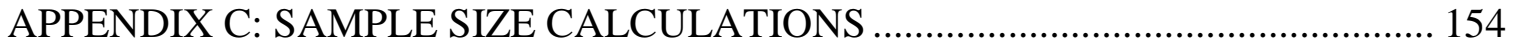

APPENDIX D: ANOVA TABLES FOR MODEL VALIDATION ........................... 156

APPENDIX E: PARTICIPANTS' ANTHROPOMETRIC DATA............................. 158

APPENDIX F: RAW GRF DATA OF RIGHT AND LEFT FOOT …........................ 160

APPENDIX G: RAW DATA OF MAJOR JOINT ANGLES ................................... 167

APPENDIX H: RAW DATA OF LOW BACK AND SHOULDER JOINT LOADING

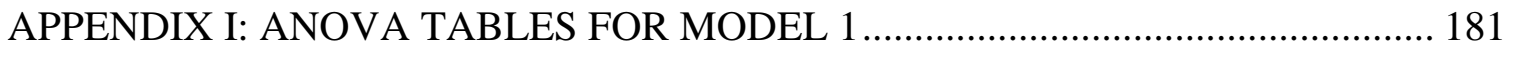

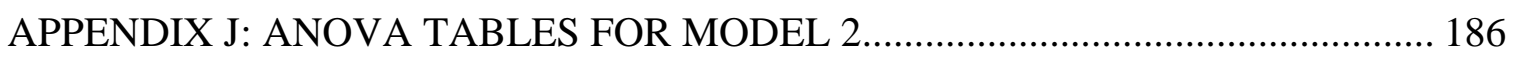

APPENDIX K: ALL-PAIRWISE COMPARISON TEST TABLES FOR MODEL 2 .. 192

APPENDIX L: REGRESSION ANALYSIS FOR PROBLEMATIC LOADING......... 199 


\section{LIST OF FIGURES}

Figure 2.1: Pushing and pulling of two-wheeled waste container (Laursen \& Schibye, 2002) 10

Figure 2.2: Typical trolley handling on aircraft (Jäger, et al., 2007) ............................. 10

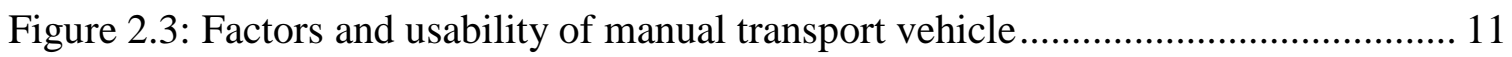

Figure 2.4: The screenshot of 3DSSPP window after the posture was entered (University

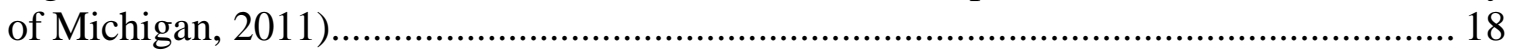

Figure 2.5: An example of the EMG-assisted model (Marras \& Granata, 1997b) .......... 21

Figure 4.1: Equipment used for data collection, processing and analysis ....................... 35

Figure 4.2: Hand truck used in this study attached with four reflective markers ............ 36

Figure 4.3: Wooden walkway: (a) flat; (b) $5^{\circ} \mathrm{ramp}$; (c) $10^{\circ} \mathrm{ramp}$............................... 38

Figure 4.4: (a) Scissor lift table; (b) hydraulic bottle jacks to minimize the GRF data

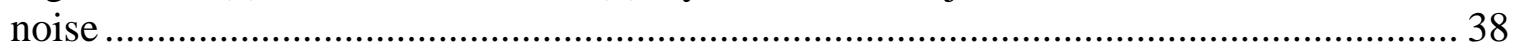

Figure 4.5: Two force plates embedded in wooden platform ..................................... 39

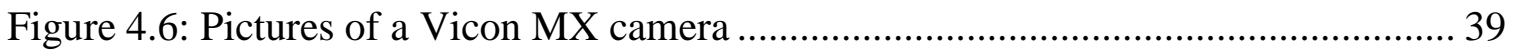

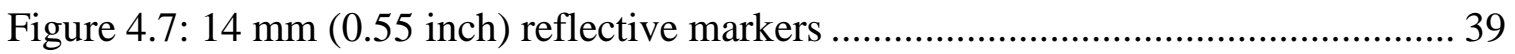

Figure 4.8: Marker locations used in the Plug-in Gait (PIG) marker set ........................ 41

Figure 4.9: Inverse dynamic analytical process implemented by AnyBody modeling

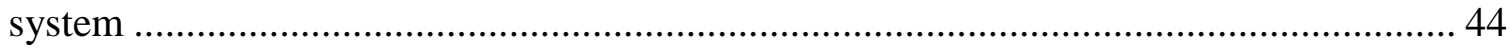

Figure 4.10: (a) Static calibration object - "L frame"; (b) $240 \mathrm{~mm}$ camera calibration wand 46

Figure 4.11: Participant performing cart pushing/pulling tasks along (a) a flat walkway, (b) a ramped walkway while going uphill, and (c) a ramped walkway while going downhill 48

Figure 4.12: The human model performing cart pushing/pulling tasks created by Vicon Nexus software:(a) pushing along the flat walkway; (b) uphill pushing along a ramp; (c) downhill pulling along a ramp.... 50

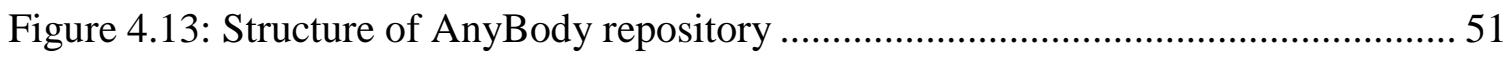

Figure 4.14: Structure of the musculoskeletal model and details of the kinematic and

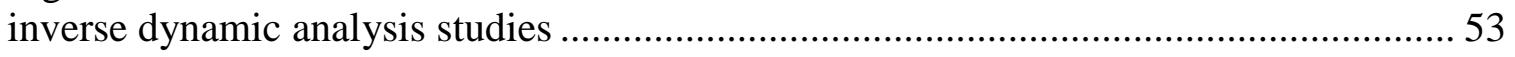

Figure 4.15: Model statement for defining the four markers attached on the cart........... 55

Figure 4.16: Model statement for parameter optimization control ............................... 55

Figure 4.17: Model statements for controlling file name, start and ending time............. 56

Figure 4.18: Model statement for inputting individual anthropometric measurements.... 57 
Figure 4.19: Model statement for initial position and posture for participant

Figure 4.20: Model statement for definition and visualization of the hand cart used in the study

Figure 4.21: Cart model and hand/cart interface visualized in AnyBody modeling system

Figure 4.22: Model statement for the hand/cart interface formulation

Figure 4.23: Model statement for auto detection of force plate touched by the foot steps64

Figure 4.24: Model statement for automatic saving the motion data of the trunk..... 65

Figure 4.25: Model statement for automatic saving the cart motion data

Figure 4.26: Model statement for running the optimized trunk motion data in inverse dynamic analysis step .....

Figure 4.27: Model statement for running the optimized cart motion data in inverse dynamic analysis step 66

Figure 4.28: The biomechanical model used for quantifying the musculoskeletal loading created by the AnyBody modeling system: (a) pushing along the flat walkway; (b) uphill pushing along a ramp; (c) downhill pulling along a ramp....

Figure 5.1: (a) Cart wheel is moving over the force plates; (b) the participant is stepping on the force plates 71

Figure 5.2: Kinematics data processed by the Vicon Nexus software, corresponding to the experimental trial shown in Figure 5.1(a)....

Figure 5.3: The originally stored Analog data of cart wheels and human body shown by C3Deditor in Windows ....

Figure 5.4: The modified Analog data after zeroing the cart wheel forces shown by C3Deditor in Windows ..... 73

Figure 5.5: Model statement of computing cart wheels' vertical reaction forces..... 74

Figure 5.6: Picture of the musculoskeletal model used for computing cart wheels' vertical reaction forces

Figure 5.7: (a) - (c) The pattern of the actual and computed wheel reaction forces during pushing tasks performed at 20,30 , and $40 \mathrm{~kg}$ weight conditions on a flat walkway and the corresponding absolute error for averaged across the participants ..... 79

Figure 5.8: (d) - (f) The pattern of the actual and computed wheel reaction forces during uphill pushing tasks performed at 20,30 , and $40 \mathrm{~kg}$ weight conditions on a gradient of $5^{\circ}$ and the corresponding absolute error for averaged across the participants .... 80

Figure 5.9: (g) - (i) The pattern of the actual and computed wheel reaction forces during downhill pulling tasks performed at 20,30 , and $40 \mathrm{~kg}$ weight conditions on a gradient of $5^{\circ}$ and the corresponding absolute error for averaged across the participants. 81 
Figure 5.10: (j) - (1) The pattern of the actual and computed wheel reaction forces during uphill pushing tasks performed at 20,30, and $40 \mathrm{~kg}$ weight conditions on a gradient of $10^{\circ}$ and the corresponding absolute error for averaged across the participants..... 82

Figure 5.11: (m) - (o) The pattern of the actual and computed wheel reaction forces during downhill pulling tasks performed at 20,30 , and $40 \mathrm{~kg}$ weight conditions on a gradient of $10^{\circ}$ and the corresponding absolute error for averaged across the participants

Figure 6.1: Peak reaction forces acting at the L5S1 joint in three anatomical directions as a function of load weight .88

Figure 6.2: Peak reaction forces acting at the sternoclavicular (SC) joint in three anatomical directions as a function of load weight. 89

Figure 6.3: Peak reaction forces acting at the acromioclavicular (AC) joint in the three anatomical direction as a function of load weight 90

Figure 6.4: Peak reaction forces acting at the glenohumeral $(\mathrm{GH})$ joint in three anatomical directions as a function of load weight

Figure 6.5: Main effect plots for the loading of the L5S1 joint in three directions ((a) L5S1-ML; (b) L5S1-PD; (c) L5S1-AP) by the walkway gradient, load weight, and direction of force application

Figure 6.6: Two-way interaction plots for the loading of the L5S1 joint in three directions: (a) L5S1-ML; (b) L5S1-PD; (c) L5S1-AP... 93

Figure 6.7: Three-way interaction plots for the loading of the L5S1 joint unstacked by the walkway gradient (degree) factor 95

Figure 6.8: Peak force acting at the L5S1 joint in the medio-lateral direction during uphill pushing and downhill pulling 96

Figure 6.9: Peak force acting at the L5S1 joint in the compression (proximal-distal) direction during uphill pushing and downhill pulling....

Figure 6.10: Peak force acting at the L5S1 joint in the anterior-posterior direction during uphill pushing and downhill pulling 98

Figure 6.11: Main effect plots for the loading of the sternoclavicular (SC) joint in three directions ((a) RSC-ML; (b) RSC-IS; (c) RSC-AP) by the walkway gradient, load weight, and direction of force application

Figure 6.12: Two-way interaction plots for the loading of the sternoclavicular (SC) joint in three directions: (a) RSC-ML; (b) RSC-PD; (c) RSC-AP 101

Figure 6.13: Three-way interaction plots for the loading of the sternoclavicular (SC) joint unstacked by the walkway gradient (degree) factor 103

Figure 6.14: Peak force acting at the sternoclavicular (SC) joint in the medio-lateral direction during uphill pushing and downhill pulling. 104

Figure 6.15: Peak force acting at the sternoclavicular (SC) joint in the inferior-superior direction during uphill pushing and downhill pulling..... 105 
Figure 6.16: Peak force acting at the sternoclavicular (SC) joint in the anterior-posterior direction during uphill pushing and downhill pulling. 106

Figure 6.17: Main effect plots for the loading of the acromioclavicular (AC) joint in three directions ((a) RAC-ML; (b) RAC-IS; (c) RAC-AP) by the walkway gradient, load weight, and direction of force application .....

Figure 6.18: Two-way interaction plots for the loading of the acromioclavicular (AC) joint in three directions: (a) RAC-ML; (b) RAC-IS; (c) RAC-AP 108

Figure 6.19: Three-way interaction plots for the loading of the acromioclavicular (AC) joint unstacked by the walkway gradient (degree) factor ..... 110

Figure 6.20: Peak force acting at the acromioclavicualr (AC) joint in the medio-lateral direction during uphill pushing and downhill pulling.... 111

Figure 6.21: Peak force acting at the acromioclavicualr (AC) joint in the inferior-superior direction during uphill pushing and downhill pulling..... 112

Figure 6.22: Peak force acting at the acromioclavicualr (AC) joint in the anteriorposterior direction during uphill pushing and downhill pulling

Figure 6.23: Main effect plots for the loading of the glenohumeral $(\mathrm{GH})$ joint in three directions ((a) RGH-DIS; (b) RGH-IS; (c) RGH-AP) by the walkway gradient, load weight, and direction of force application 114

Figure 6.24: Two-way interaction plots for the loading of the glenohumeral $(\mathrm{GH})$ joint in three directions: (a) RGH-DIS; (b) RGH-IS; (c) RGH-AP 115

Figure 6.25: Three-way interaction plots for the loading of the glenohumeral (GH) joint unstacked by the walkway gradient (degree) factor

Figure 6.26: Peak force acting at the glenohumeral $(\mathrm{GH})$ joint in the distraction direction during uphill pushing and downhill pulling.... 118

Figure 6.27: Peak force acting at the glenohumeral $(\mathrm{GH})$ joint in the inferior-superior direction during uphill pushing and downhill pulling.....

Figure 6.28: Peak force acting at the glenohumeral $(\mathrm{GH})$ joint in the anterior-posterior direction during uphill pushing and downhill pulling. 120 


\section{LIST OF TABLES}

Table 4.1: Dependent variables used for studying shoulder joint loading ...................... 30

Table 4.2: Power values for different number of subjects for Model 1 ........................... 34

Table 4.3: Power values for different number of subjects for Model 2 .......................... 34

Table 4.4: Anatomical locations of Plug-in Gait marker set ....................................... 42

Table 4.5: Required subject anthropometric measurements ...................................... 47

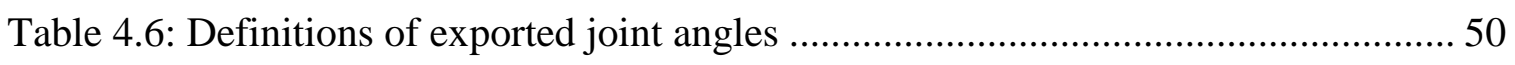

Table 5.1: Accuracy of the computed left cart wheel's vertical forces as represented by the correlation coefficient $r$ and the root mean square error (RMSE), and the peak forces

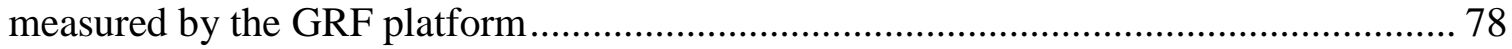

Table 6.1: Participants' demographic and anthropometric data .................................. 84

Table 6.2: Mean (SD) of the peak ground reaction force (GRF) across all experimental

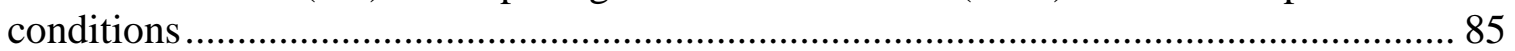

Table 6.3: The minimum, maximum and mean of peak joint angles (in degrees) for trunk, right upper and lower limbs across all experimental conditions ......................... 87

Table 6.4: Mean (SD) of the peak forces acting at the L5S1 joint during pushing task

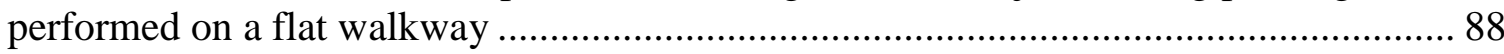

Table 6.5: Mean (SD) of the peak forces acting at the sternoclavicular (SC) joint during

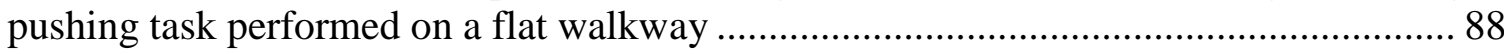

Table 6.6: Mean (SD) of the peak forces acting at the acromioclavicular (AC) joint

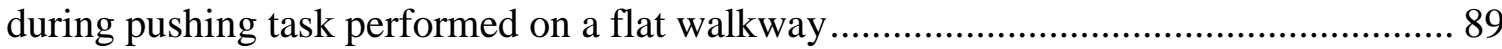

Table 6.7: Mean (SD) of the peak forces acting at the glenohumeral $(\mathrm{GH})$ joint during pushing task performed on a flat walkway 


\section{CHAPTER 1: INTRODUCTION}

Work-related musculoskeletal disorders (MSD) are among the most costly health problems facing society today (National Research Council and Institute of Medicine, 2001). In the United States, MSD impose a substantial economic burden in terms of worker compensation costs, lost wages, and decreased productivity. Conservative cost estimates vary, but a reasonable number is between $\$ 45$ and $\$ 54$ billion annually (Dunning, et al., 2010). According to the Bureau of Labor Statistics (2010), MSD among the occupational groups such as laborers and material movers, heavy and tractor-trailer truck drivers, and delivery service truck drivers, whose routine work demands manual material handling $(\mathrm{MMH})$ tasks, constitute $12 \%$ of all MSD cases (144,910 out of $1,238,490$ cases) requiring days away from work. The body parts that are most affected by $\mathrm{MMH}$ exertions include neck, upper extremities, and low back. Although neck and shoulder MSD are not as prominent as low back, these disorders contribute significantly to morbidity among various occupational groups and also put a substantial burden in terms of health and economics (Nonnenmann, et al., 2008; Hess, et al., 2010; Holmström, Lindell, \& Moritz, 1992; Grooten, et al., 2004; Cassou, et al., 2002).

Manual material handling (MMH) tasks commonly performed at the workplaces primarily are comprised of activities such as lifting/lowering, pushing/pulling, and carrying loads (Snook, 1978; Smith, Woldstad, \& Patterson, 2009). Traditionally, most of the exertions at workplaces that were linked with the physical risk factors such as high force and repetitive exertions were primarily during the lifting and lowering tasks. However, the workplace and the nature of the work are changing rapidly. Recently, most manufacturing is done offshore. With the introduction of e-commerce, a vast increase in distribution center jobs has also occurred where order picking with the use of cart pushing/pulling is becoming a common task for many workers. Collectively, these trends indicate that the nature of physical exposure is rapidly evolving to a highly repetitive environment where the repetition may involve different vectors of force application (Marras, et al., 2009). It has been estimated that nearly half of MMH tasks common at workplaces demand pushing and pulling exertions (Lee \& Granata, 2006). In addition to 
manufacturing workers, other occupations that demand pushing and pulling types of exertions on a daily basis include health care (Waters, Nelson, \& Proctor, 2007; Seto, et al., 2006; Das \& Wimpee, 2002), fire fighting (Gledhill \& Jamnik, 1992), flight and rail catering (Glitsch, et al., 2007; Schaub, et al., 2007; Jäger, et al., 2007), postal distribution (Hoozemans, et al., 2007; Hoozemans, et al., 2004; van der Beek, et al., 2000), refuse collection (Schibye, Søgaard, et al., 2001; Frings-Dresen, Kemper, Stassen, Markslag, et al., 1995; Frings-Dresen, Kemper, Stassen, Crolla, et al., 1995), construction (Søgaard, et al., 2001) and garden raking (Kumar, 1995), retail, storage, and warehousing (Kumar, Narayan, \& Bacchus, 1995).

Pushing and pulling tasks are characterized by the force exertions with the direction of the resultant force being horizontal. In pushing, the force is directed away from the body, and in pulling, the force is directed toward the body. According to Chaffin (1987), the risk of health complaints induced by pushing and pulling can arise from two types of hazards. Firstly, the musculoskeletal system can become physically over-exerted. Secondly, pushing and pulling is accompanied by an increased risk of accidents due to slipping or tripping, which can cause injuries to the musculoskeletal system. Among the prior type, an association between the work-related pushing/pulling exertions and complaints of low back pain is reported in a number of studies (Hoozemans, et al., 1998; van der Beek, et al., 1993; Ozguler, et al., 2000; Klein, Jensen, \& Sanderson, 1984; Harber, et al., 1987; Garg \& Moore, 1992; Fuortes, et al., 1994; Frymoyer, et al., 1980; Damkot, et al., 1984). Pushing and pulling types of exertion are also implicated as a risk factor for the proliferation of MSD of the shoulder region and other upper extremities (Hoozemans, et al., 1998; van der Beek, et al., 1993; Smedley, et al., 2003; Hoozemans, et al., 2002a, 2002b). Given recent advances in research and the changing work environment, the National Institute for Occupational Safety and Health (NIOSH) addressed in its national occupational research agenda (NORA) that "...Efforts to quantitatively link epidemiological, biomechanical loading, soft tissue tolerance, and psychosocial factors studies should be pursued to establish a better understanding of the pathways of injury and resultant preventive strategies.” (Marras, et al., 2009) 
To gain insight into the causal relationship between low back and shoulder MSD and pushing/pulling exertions, work-related pushing and pulling exertions were evaluated in a number of experimental studies. Early pushing/pulling studies focused predominantly on evaluation of static exertions (Chaffin, Andres, \& Garg, 1983; Kroemer, 1974; Ayoub \& Mcdaniel, 1974). The recent studies that evaluated dynamic exertions primarily focused on investigating changes in the magnitude of hand forces corresponding to different pushing/pulling techniques (Todd, 2005; Resnick \& Chaffin, 1995; Bennett, et al., 2008) and the effect of pushing/pulling exertions on various physiological parameters, such as heart rate and oxygen uptake, etc. (van der Beek, et al., 2000; Frings-Dresen, Kemper, Stassen, Markslag, et al., 1995; Frings-Dresen, Kemper, Stassen, Crolla, et al., 1995; van der Beek \& Frings-Dresen, 1995). Only a few studies looked at the effect of dynamic pushing/pulling exertions on the musculoskeletal loading (Jäger, et al., 2007; Hoozemans, et al., 2004; Schibye, Søgaard, et al., 2001; Laursen \& Schibye, 2002). Within industrial settings, dynamic pushing and pulling exertions performed on flat or inclined walkways are highly prevalent. Dynamic movement imposes different demands on the musculoskeletal system and several biomechanical models and epidemiologic studies suggest that the dynamic work is associated with a higher risk of injury compared to static work (Allread, Marras, \& Burr, 2000; Marras, et al., 1993).

Currently, it is not clearly understood as to how the dynamic pushing/pulling exertions performed under different task conditions (e.g. weight, walkway gradient, direction of force application) affect the musculoskeletal loading of low back and shoulder joints. Quantification of three-dimensional (3D) loads experienced by certain joints (e.g., the spine and shoulder joints) is essential to better understand the pathways of injury and to develop adequate intervention strategies. Therefore, the purpose of the present study was to evaluate dynamic pushing and pulling tasks performed under different workplace conditions to quantify their effects on the musculoskeletal loading of major low back and shoulder joints. The tasks evaluated in this study were pushing a cart with loads of 20,30 , and $40 \mathrm{~kg}$ on a level walkway and while going uphill along $5^{\circ}$ and $10^{\circ}$ ramps. In addition, pulling tasks while going downhill were also evaluated. An eightcamera optical motion system configured with two ground reaction force plates was used 
for data collection. The experimental data were exported in the C3D format, which were then used for running a full-body musculoskeletal model to compute the biomechanical loading in terms of joint reaction forces in three anatomical directions acting at the L5S1 joint of low back and three shoulder joints (sternoclavicular, acromioclavicular and glenohumeral joints) in the shoulder complex. 


\section{CHAPTER 2: LITERATURE REVIEW}

\subsection{Introduction}

Manual material handling $(\mathrm{MMH})$ tasks consist of a wide range of activities including lifting, lowering, carrying, pushing, and pulling (Snook, 1978; Smith, et al., 2009) and have long been a focus for a diverse range of disciplines as a result of the large economic and human cost of injuries incurred by workers involved in MMH (Mital, Nicholson, \& Ayoub, 1997). Workplace pushing and pulling exertions involve the use of trolleys, pallet jacks, and other wheeled devices that allow for the movement of a larger quantity of goods (van der Beek, et al., 1999). Pushing/pulling tasks performed using a host of manual transport aids may seem relatively safe in terms of musculoskeletal loading, however the literature indicates that with pushing/pulling activities, the nature of risk is changed rather than eliminated (Resnick \& Chaffin, 1995). Work-related pushing/pulling activities are generally identified amongst the risk factors associated with musculoskeletal disorders such as low back pain, shoulder stiffness, and upper extremity complaints (Hoozemans, et al., 1998).

In this chapter, studies evaluating pushing and pulling tasks are reviewed. The studies are classified into five sections: (1) fundamental concepts of work-related pushing and pulling exertions; (2) relationship between work-related musculoskeletal disorders and pushing/pulling exertions; (3) static and dynamic pushing/pulling exertions; (4) factors affecting pushing/pulling tasks with a focus on the effect of load weight, walkway gradient, and direction of exerted forces; and (5) musculoskeletal models evaluating pushing/pulling exertions.

\subsection{Work-Related Pushing and Pulling Exertions}

In general, pushing and pulling could be defined as the exertion of a force using the hand where direction of the resultant force is horizontal (Hoozemans, et al., 1998). However, depending on the location of the hands, it is necessary to note that an exertion of force is not always directed horizontally, for instance, in pulling a cord to start a lawn 
mower engine, the force is directed vertically (Garg, Funke, \& Janisch, 1988). In the present study, "uphill pushing" and "downhill pulling" exertions along an inclined walkway with the direction of the resultant force being horizontal were considered, where force is directed away from the body during pushing and toward the body during pulling.

\subsection{Musculoskeletal Disorders: Pushing and Pulling}

Numerous studies have reported that the risk of health complaints induced by pushing and pulling can arise from two types of hazards (Hoozemans, et al., 1998). First,

the musculoskeletal system can become physically over-exerted due to excessive, too long, or too frequent force applications (Glitsch, et al., 2007). Second, pushing and pulling are accompanied by additional dangers from slipping and tripping when maneuvering hand-propelled transport vehicles, which can cause acute injuries to the musculoskeletal system (Glitsch, et al., 2007; Grieve, 1983). In the present study, the focus was on understanding how the musculoskeletal loading was altered with the changes in the load weight transferred and other workplace characteristics so that the pathomechanism of musculoskeletal injuries caused by the physical overexertion could be understood.

Many authors have suggested pushing and pulling as an occupational risk factor for low back pain (Hoozemans, et al., 1998; van der Beek, et al., 1993; Ozguler, et al., 2000; Klein, et al., 1984; Harber, et al., 1987; Garg \& Moore, 1992; Fuortes, et al., 1994; Frymoyer, et al., 1980; Damkot, et al., 1984; NIOSH, 1997, 1981). NIOSH (1997) reported that $52 \%$ of the injuries leading to the back pain were associated with pushing and pulling. Garg and Moore (1992) found that work-related pushing and pulling activities account for 9-18\% of all back strains and sprains. Harber et al. (1987) investigated the relationship between back pain and work factors among hospital nurses by using survey questionnaires. Carrying and pushing were combined in one factor and were significantly related to back pain. In another questionnaire study performed among Dutch workers, it was found that workers who frequently pushed or pulled objects at work reported low back pain more often than those who did not (Hoozemans, et al., 1998) . 
In addition to the low back MSD, several studies have also claimed that pushing and pulling exertions are associated with shoulder complaints (Hoozemans, et al., 1998; van der Beek, et al., 1993; Smedley, et al., 2003; Hoozemans, et al., 2002a, 2002b; Hughes, Silverstein, \& Evanoff, 1997). Van der Beek et al. (1993) investigated the prevalence of musculoskeletal complaints in lorry drivers and found significantly a higher number of neck, shoulder and upper and lower extremity complaints in the lorry drivers who regularly pushed or pulled wheeled cages during the working day compared to those who only performed driving tasks. In an another study by Smedley et al. (2003), patient handling tasks that demand reaching, pushing, and pulling were identified as the physical risk factors for neck and shoulder MSD. Hughes et al. (1997) identified pushing and pulling tasks as a risk factor for shoulder MSD among the aluminum smelter workers. A dose-response relationship between the work-related pushing/pulling exertions and complaints of shoulder pain was also reported by Hoozemans et al. (2002a, 2002b).

The common work-related pathologies associated with the shoulder complex include: shoulder tendinitis, rotator cuff pain, and nonspecific shoulder pain. Repetitive loading of shoulder joints has been identified as one of the causal factors for these shoulder complex pain syndromes (NIOSH, 1997). The shoulder mechanism provides the upper limb with an extensive range of movement exceeding that of any other joint mechanism. The shoulder complex consists of three joints: sternoclavicular (SC), acromioclavicular (AC), and glenohumeral (GH) joints and the scapulothoracic gliding mechanism.

The SC joint, the only synovial articulation between the upper limb and the axial skeleton, has three degrees of freedom of movement. Because the distal end of the clavicle is attached to the $\mathrm{AC}$ joint, all movements of the clavicle are accompanied by movements of the scapula. Motions at the SC joint consist of: elevation/depression, protraction/retraction, and rotation (Reese, Bandy, \& Yates, 2009). The AC joint is classified as a plane synovial joint and is formed by the articulation of the acromial end of the clavicle with the medial border of the acromion process of the scapula. The AC joint has been described as having three degrees of freedom of movement. These 
movements are rotation, winging, and tilting, which occur during scapular motions of rotation, abduction/adduction, and elevation/depression, respectively (Reese, et al., 2009). The GH joint is a synovial ball-and-socket joint between the humeral head and the glenoid fossa of the scapula. The shallowness of the glenoid fossa and the disproportionate size and lack of congruency of the articular surfaces make the joint inherently unstable (Culham \& Peat, 1993). This relative instability of the GH joint allows higher freedom of movement, permitting placement of the upper extremity in a wide variety of positions for function. The joint possesses three degrees of freedom, allowing the motions of flexion/extension, abduction/adduction, and medial/lateral rotation (Reese, et al., 2009). In addition to the three joints, a scapulothoracic and a subacromial joint are often included in anatomical descriptions of the shoulder complex. Together, these articulations provide the shoulder a wide range of mobility, together with elbow motion, allows positioning of the hand anywhere, which may cause overuse injuries for the shoulder complex.

\subsection{Static and Dynamic Exertions}

Pushing and pulling tasks common at workplaces may involve static and/or dynamic exertions, or a combination of both, with most dynamic efforts including a static component usually in the trunk and upper extremities (Garcin, et al., 1996). Static exertions most often involve supporting/controlling fixed objects or apparatus, resulting in the force exertion against a stationary target (Lee, et al., 1991). Conversely, dynamic exertions usually involve force exertion to transport loads, causing object displacement (Lee, et al., 1991).

Static pushing/pulling tasks have historically been at the core of scientific investigations that evaluated either maximum push/pull forces (Chaffin, et al., 1983; Kroemer, 1974; Ayoub \& Mcdaniel, 1974) or low back muscle forces (Lee, et al., 1989), while pushing/pulling tasks in industry are predominantly dynamic (Todd, 2005; Bennett, et al., 2008). The differences between static and dynamic activities are mainly in terms of posture and force generation. For the same level of effort, body postures vary between the two, impacting on low back loading and implying that the ceiling parameters derived 
statically may not be applicable to dynamic exertions. This was acknowledged by Resnick and Chaffin (1995) who stated that postures and forces change rapidly during dynamic pushing/pulling, and as such, optimal postures are not practicable for the duration of the task. Under low accelerations, peak pushing forces may approximate those of static efforts, suggesting that static estimations may be used to predict peak pushing forces at low accelerations, but when accelerations are high, dynamic standards will be necessary (Resnick \& Chaffin, 1995). Some authors have advocated that static standards are inappropriate for evaluating dynamic exertions, considering that dynamic push/pull strength, being significantly lower than static ones (Kumar, et al., 1995; Mital, Kopardekar, \& Motorwala, 1995).

Considering the prevalence of dynamic pushing/pulling exertions at workplaces (Lee, et al., 1991), a few studies have investigated dynamic pushing and pulling tasks, such as using two-wheeled waste containers (see Figure 2.1) (Schibye, Søgaard, et al., 2001; Laursen \& Schibye, 2002), collecting refuse in two-wheeled mini-containers or in large four-wheeled containers (Frings-Dresen, Kemper, Stassen, Markslag, et al., 1995; Frings-Dresen, Kemper, Stassen, Crolla, et al., 1995), wheeled cages used by postal workers (van der Beek, et al., 2000) or lorry drivers (van der Beek \& Frings-Dresen, 1995), four-wheeled aircraft trolleys (see Figure 2.2) (Glitsch, et al., 2007; Jäger, et al., 2007), or standard four-wheeled carts as used in postal distribution centers (Hoozemans, et al., 2007; Hoozemans, et al., 2004). The primary outcome variables evaluated in these studies include hand forces, physiological parameters such as heart rate and oxygen uptake, two-dimensional (2D) loading of shoulder and lumbar spine, and threedimensional (3D) loading of lumbar spine. Some of these studies will be revisited in the following sections. 

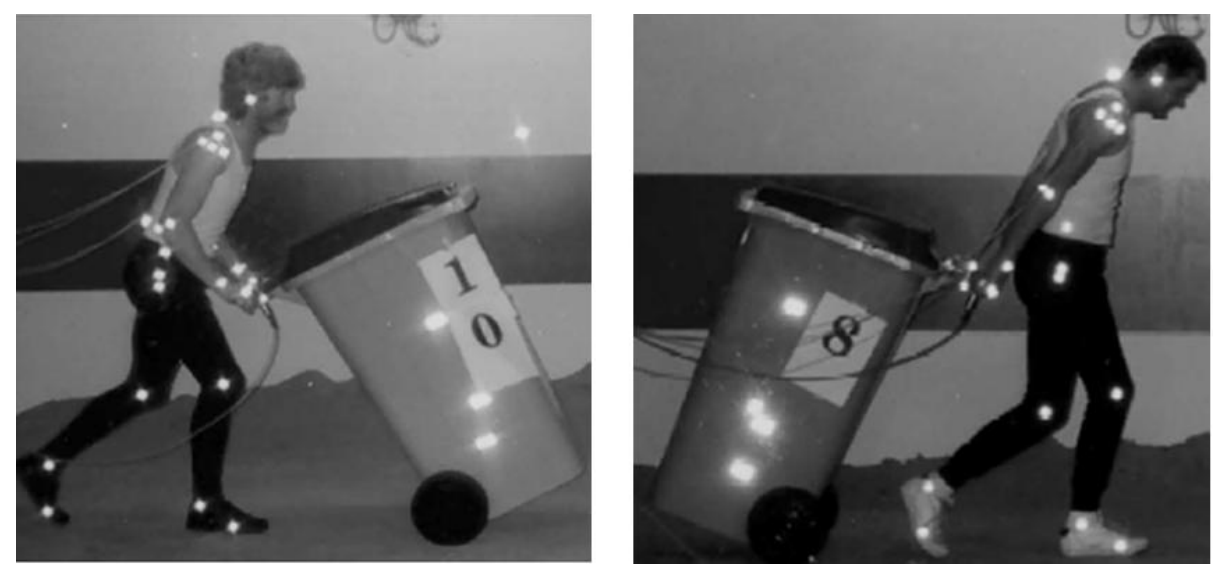

Figure 2.1: Pushing and pulling of two-wheeled waste container (Laursen \& Schibye, 2002)
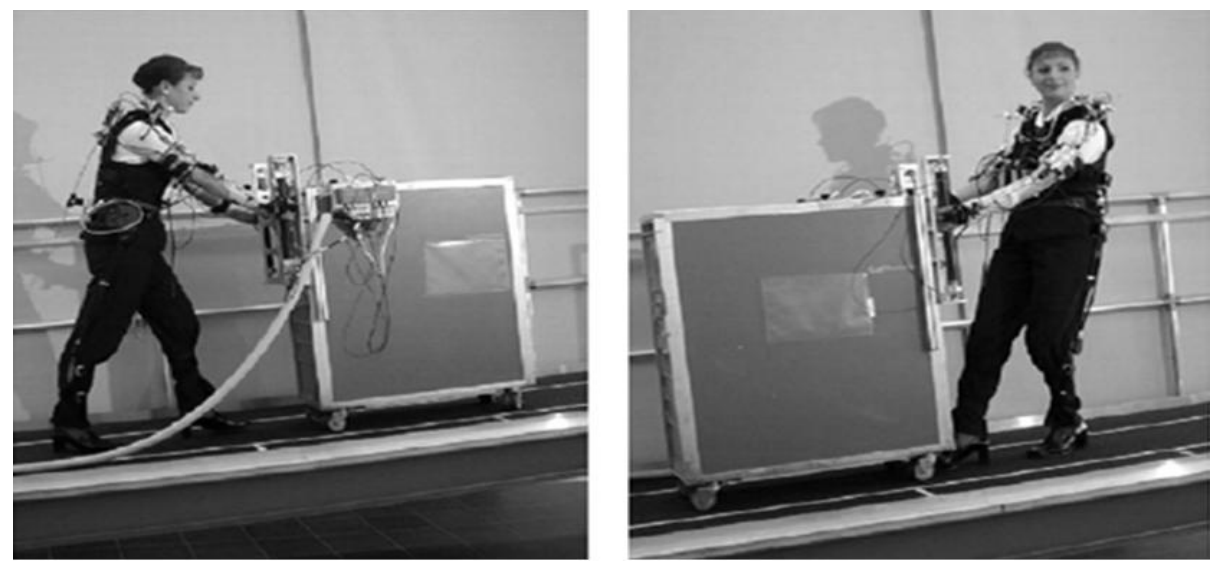

Figure 2.2: Typical trolley handling on aircraft (Jäger, et al., 2007)

\subsection{Factors Affecting Pushing and Pulling}

Pushing/pulling tasks performed using manual transport vehicles are affected by a number factors and a multifaceted interaction between them. These factors have been broadly categorized into design, environmental, task, and operator factors, as shown in Figure 2.3 (Jung, Haight, \& Freivalds, 2005). The present study was aimed to investigate the effect of load weight, walkway gradient, and direction of force exertion on the musculoskeletal loading during dynamic pushing/pulling tasks performed using a twowheeled hand truck. In terms of the factors most frequently considered important in a real industrial setting for pushing/pulling tasks, the effect of task factors (load weight and direction of force application) and environmental factors (walkway gradient) has not yet 
been well studied in the literature. The literature related to the three aforementioned factors will be reviewed in the following sections.

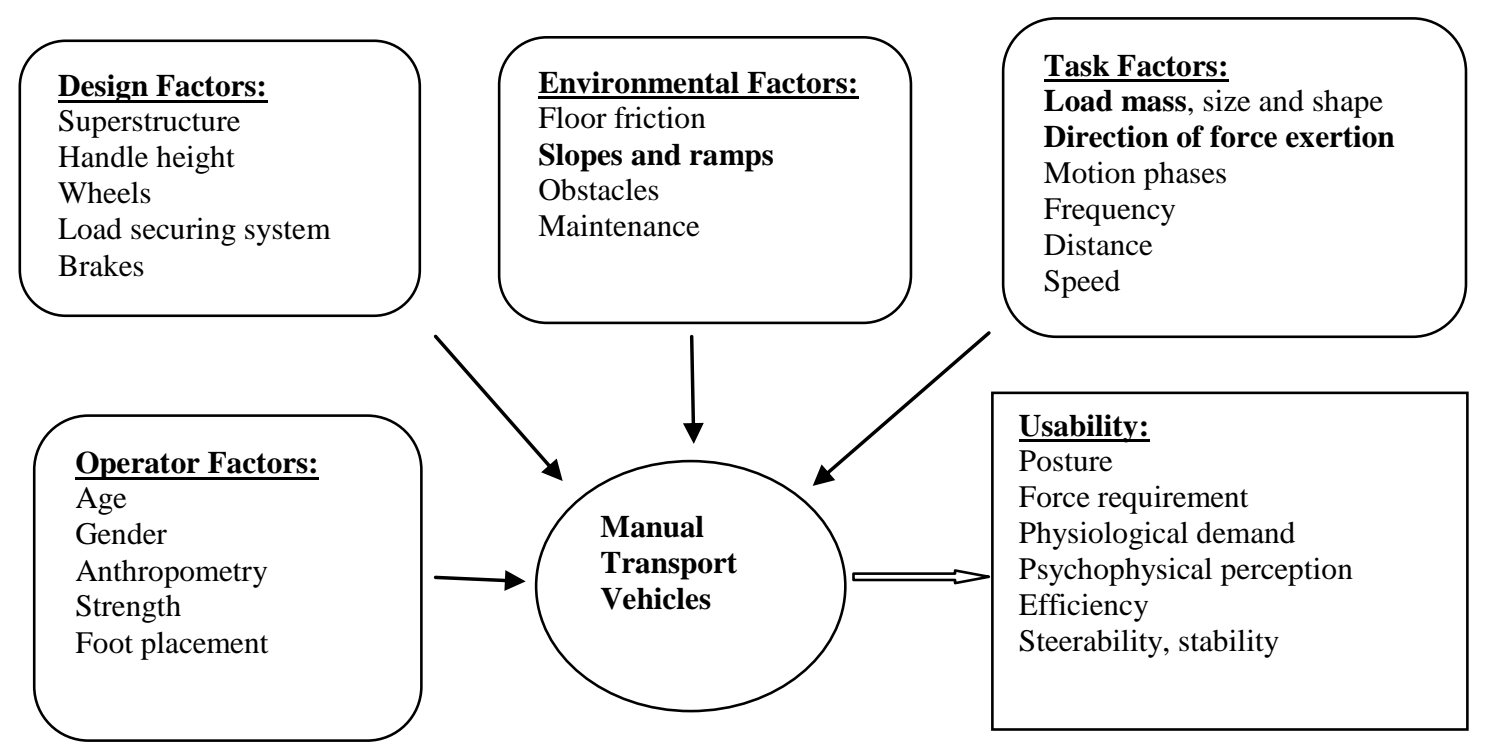

Figure 2.3: Factors and usability of manual transport vehicle

\subsubsection{Load weight}

Load weight is one of the principal factors to be considered in the (re)design of push/pull tasks, because it is known to greatly affect force output and the loading of the musculoskeletal system (Resnick \& Chaffin, 1995; Bennett, et al., 2008; Jung, et al., 2005). A survey of several industries by Mack et al. (1995) found a high variability in the load weight transferred using mechanical transfer aides by pushing/pulling actions. The load transferred per trip varied from $2 \mathrm{~kg}$ to $1500 \mathrm{~kg}$ on a hand pallet truck with distances varying from a few meters to hundreds of meters. Frings-Dresen et al. (1995) found that the weight of loads moved by refuse collectors, with a single individual, displaced average loads of $22 \mathrm{~kg}$ and $110 \mathrm{~kg}$ using mini and large containers, respectively. While a more recent study by St-Vincent et al. (2005) concluded that in a superstore, the total cumulative load moved using a pallet jack on a daily basis may be as high as $4400 \mathrm{~kg}$. Despite the variability of the masses being moved, many authors have argued that regardless of vehicle type, load weight should be minimized to prevent excessive bodily stress (van der Beek, et al., 2000; Resnick \& Chaffin, 1995), with the load's center of mass situated as low as possible (Kingma, et al., 2003). 
In the study of Schibye et al. (2001), two loads (25 and $50 \mathrm{~kg}$ of waste) were used for the experiments, representing a filled and a heavily overfilled container, respectively. The container was pushed in front of and pulled behind the body by seven refuse collectors. A biomechanical analysis was made by using the Watbak system (University of Waterloo, Waterloo, Canada). The biomechanical calculations were based on a quasistatic 2D linked segment model. The results showed that during pushing and pulling of the two-wheeled container, the compression force at L4/L5 in all situations was rather small (605-1445 N) and never exceeded the value of $3400 \mathrm{~N}$ proposed as an acceptable limit by NIOSH (Waters, et al., 1993). The compression was smaller during pushing than during pulling. The compression was not affected by an increase in the amount of waste in the container. The shear forces at L4/L5 were generally small with the highest average value $(200 \mathrm{~N})$ obtained during pushing of the container. The external shoulder torque at the shoulder joint during pushing and pulling varied from -35 to $38 \mathrm{Nm}$. Flexion torques were positive during pushing and extension torques negative during pulling. Torque at the shoulder was larger during pushing or pulling of $50 \mathrm{~kg}$ compared to $25 \mathrm{~kg}$.

In a study performed by Laursen and Schibye (2002), seven male waste collectors pushed and pulled a two-wheeled container on three different surfaces: flagstones, paving stones, and grass. The container was filled with 1, 2, or 4 newspaper bundles each weighing $12.5 \mathrm{~kg}$. The biomechanical calculations were performed by software based on a 2D static linked segment model of the human body (Norman \& McGill, 1993). The results showed that the lumbar spine compression force was below $1800 \mathrm{~N}$ and the shear force was below $200 \mathrm{~N}$ in all situations. The shoulder torque when pulling with one hand was up to $80 \mathrm{Nm}$. The container weight affected the magnitude of the push/pull forces and the load on the shoulders but not the load on the lumbar spine. The type of surface affected the magnitude of the push/pull forces during initial and sustained phases and affected the load on the shoulder in the sustained phase. However, it did not affect the compression in the lumbar spine.

With the use of a standard four-wheeled cart, Hoozemans et al. (2004) examined the effect of pushing and pulling three load weights, 85, 135, and $320 \mathrm{~kg}$ on the exerted forces and the mechanical load on the low back and shoulders. An upper body quasi- 
dynamic 3D linked segment model was used to perform the biomechanical calculations. Results indicated that load weight affected each of the dependent variables (exerted forces, net moments, and compression and shear forces) significantly, i.e., an increase in load weight resulted in a significant increase of all dependent variables. For pushing or pulling at hip height, the predicted maximum compression force increased with $5 \mathrm{~N}$ for every kilogram that was added to the weight of the cart. With respect to the $320 \mathrm{~kg}$ cart used in this study, at hip height the maximum compression force at the low back was predicted to be $1600 \mathrm{~N}$.

In the study of Bennett et al. (2008), in order to determine the impact of load on biomechanical responses during dynamic trolley pushing and pulling, participants pushed/pulled loads of 100,200 , and $300 \mathrm{~kg}$ on the level wooden walkway. When investigating load effect on hand forces, it was found that there existed a linear relationship between the two, with higher loads eliciting associated higher hand forces, particularly initial forces.

When investigating physical workload when handling trolleys on aircraft, Glitsch et al. (2007) used half-size and full-size trolleys in their study. The load mass of the trolleys ranged between $40 \mathrm{~kg}$ to $90 \mathrm{~kg}$, therefore two intermediate weight conditions of 45 and $65 \mathrm{~kg}$ were evaluated in their study. It was found that the maximum hand forces during the pushing and pulling of trolleys showed a strong dependence on the weight of the trolley. On horizontal floors, the hand forces were in the range of $70-150 \mathrm{~N}$, depending on trolley load. In the other part of the study (Jäger, et al., 2007) with the same laboratory set-up, $2500 \mathrm{~N}$ was chosen as the criterion for evaluating lumbar disc compression during trolley maneuvers. A spatial dynamic multi-segmental biomechanical model was used for quantifying the lumbar load during trolley handling. It illustrated that lumbar load clearly increased with increasing weight, indicating that the higher the load weight during pushing the trolleys, the higher the resultant lumbar-disc compression.

\subsubsection{Walkway gradient}

On one hand, a number of researchers have examined the biomechanics during normal gait on a level or inclined surface (Lay, Hass, \& Gregor, 2006; Li, Chang, Lin, et 
al., 2006; Li, Chang, Wei, et al., 2006; Prentice, et al., 2004; McIntosh, et al., 2006; Cham \& Redfern, 2004; S. Redfern \& DiPasquale, 1997; DiPasquale, 1997; Redfern, et al., 2001; Cham \& Redfern, 2002a, 2002b). These studies focused on the prevention of slip/fall accidents, whereas, studies evaluating pushing and pulling types of exertion along ramps are sparse, despite the fact that the presence of variable inclined ramps in industry complicates the situation during pushing and pulling because of the task demands required to overcome the downward effect of gravity and the inertia of the object. In the study of Glisch et al. (2007), the floor gradients were used $\left(0^{\circ}, 2^{\circ}, 5^{\circ}\right.$, and $8^{\circ}$ ), and it was found that the maximum hand forces during the pushing and pulling of trolleys showed a strong dependence on the gradient of the floor. Depending on the trolley load, the forces were $70-150 \mathrm{~N}$ on average on horizontal floors; while on an $8^{\circ}$ gradient, average maximum manual handling forces between 130 and $270 \mathrm{~N}$ were recorded, which in several cases proved to be excessively strenuous for women if the trolley was fully laden. Within the same experimental set-up, Jäger et al. (2007) found that during trolley pushing, lumbar load clearly increased with the increasing gradient. However, it was found that lumbar load did not increase with floor steepness while pulling the trolleys. For example, pulling an empty half-size trolley on a horizontal surface led on average to higher values of lumbar load than pulling it on an $8^{\circ}$ inclined floor.

Bennett et al. (2008) examined the effect of gradient $\left(0^{\circ}\right.$ and $\left.12^{\circ}\right)$ on the biomechanical responses in the form of hand forces during dynamic trolley pushing and pulling. Hand forces were found to be the highest during uphill initial and sustained phases and the downhill sustained phase. The authors suggested that sustained forces may be appropriate in determining the inherent potential risk of graded pushing; accordingly, for the sustained phase, there was no difference between uphill and downhill forces but level forces were significantly lower $(18.9 \pm 8.09 \mathrm{~N})$ than either of the other two conditions. The investigation of pushing along graded ramps indicated that for all motion phases, forces exhibited during uphill and/or downhill efforts were significantly greater than those elicited during level pushing. Specifically, uphill pushing was the most taxing during the initial phase, while downhill pushing imposed the greater stress on the musculoskeletal system during the ending phase; for the sustained phase, hand forces for 
both graded conditions were higher compared to level pushing. Hence, it is recommended that level pushing is preferable over graded pushing.

The aforementioned studies demonstrate that there is a relationship between gradient and the biomechanical load in terms of hand forces (Glitsch, et al., 2007; Bennett, et al., 2008) or lumbar-disc compression forces (Jäger, et al., 2007). Clearly, limited research is available regarding pushing and pulling along inclined walkways, despite a high prevalence of such tasks in a wide range of industrial settings.

\subsubsection{Direction of force application}

The direction of force application during the pushing/pulling was found to affect the musculoskeletal loading and has been identified as an important factor during the task assessment (van der Beek, et al., 1999; De Looze, et al., 2000). There can be four possible situations: (1) forward pushing with the vehicle in front of the operator (2) backward pulling with the vehicle in front of the operator, (3) forward pulling with the vehicle behind the operator and (4) forward pulling with the vehicle in front of the operator (on ramps). One-handed forward pulling and two-handed backward pulling are common as workers can use their body weight to assist in initial force production (Ayoub \& Mcdaniel, 1974). Li et al. (2008) proposed that the use of one or two hands during pulling is related to load, such that at low loads workers tend to pull forwards with one hand; however when the load exceeds this capacity, two handed pulling is commonly used or preferred.

Schibye et al. (2001) investigated the (1) forward pushing with the waste container in front of the body and (3) forward pulling with the container behind the body. The calculated compression forces at L4L5 were rather small in all situations (605-1445 $\mathrm{N})$. The reason that the stress on the lower back was rather small was that the forward trunk lean offsets the extension moment resulting from pushing and pulling forces. The compression force at L4L5 was smaller during pushing than during pulling. Forward pulling of carts resulted in twisted and laterally bent trunk postures (Frings-Dresen, Kemper, Stassen, Markslag, et al., 1995; Frings-Dresen, Kemper, Stassen, Crolla, et al., 1995; De Looze, et al., 1995) and required 9\% more force than forward pushing 
(Schibye, et al., 1997). It also caused greater spinal loading due to the shorter moment arm between the hand and the L4L5 disk for the extension moment that did not offset the flexion moment (Schibye, Sogaard, et al., 2001). However, forward pulling is more commonly used by operators because of better visibility, easier steering, and decreased muscle fatigue (Jung, et al., 2005).

Forward pushing was found to be less stressful than backward pulling (Jung, et al., 2005; De Looze, et al., 2000). Lee et al. (1991) indicated that backward pulling stressed the L5S1 two times higher than forward pushing. In the study of Jäger et al. (2007), forward pushing and backward pulling with the trolley in front of the body were utilized by the participants. Regardless of different surface inclinations and trolley loadings, no lumbar overload risk was found for pushing, whereas exceeding the recommended limit was frequently established for pulling the half size trolleys.

\subsection{Musculoskeletal Models for Pushing and Pulling}

The purpose of the present study was to evaluate the 3D musculoskeletal loading of major body joints and segments during dynamic pushing and pulling task. In the following section, biomechanical methods used for quantifying musculoskeletal loading during the pushing and pulling tasks are reviewed.

Direct measurement of mechanical load on the musculoskeletal system is very difficult. Biomechanical models are commonly used to predict mechanical stress on the musculoskeletal system (Hoozemans, et al., 1998). The models are relatively simple representations of the human body, because assumptions on the anatomical structures have to be made. With respect to pushing and pulling, risk factors for musculoskeletal disorders can be identified when biomechanical models predict an increase in mechanical stress. If the mechanical stress exceeds the load-bearing capacity of certain structures, the structures can get damaged and musculoskeletal disorders may develop (Kumar, 1990).

\subsubsection{Single equivalent muscle models}

Loads on the lumbar spine are mainly determined by muscle forces. Estimation of these loads is hampered by the fact that the muscle forces cannot be calculated, since the 
many muscles crossing the lumbar spine form a statically indeterminate system when submaximal net moments are produced (van Dieen \& de Looze, 1999). Biomechanically based spine models have been developed to help assess occupationally related manual material handling (MMH) tasks. One of the early static assessment models was developed by Chaffin at the University of Michigan. Both two-dimensional (2D) as well as three-dimensional (3D) static models (Chaffin and Muzaffer, 1991) have been developed to assess the risk of injury during MMH activities. In both models, the moments imposed upon the various joints of the body due to the object lifted are evaluated, assuming a static posture is representative of the instantaneous loading of the body. These models assume that a single equivalent muscle (internal force) supports the external moment about each joint. By considering the contribution of the externally applied load and the internally generated single muscle equivalent, spine compression acting on the lumbar discs is predicted. The $2 \mathrm{D}$ version of this computer model assumes that all lifts occur directly in front of the worker in the sagittal plane. Another important assumption of these models is that no significant motion occurs during the exertion because it is a static model. The 3D version of the computer model works in a similar manner; however, non-sagittal symmetric lifting assumptions are permitted. Figure 2.4 (University of Michigan, 2011) shows the output screen for this computer model where the lifting posture, lifting distances, strength predictions, and spine compression forces are shown. 


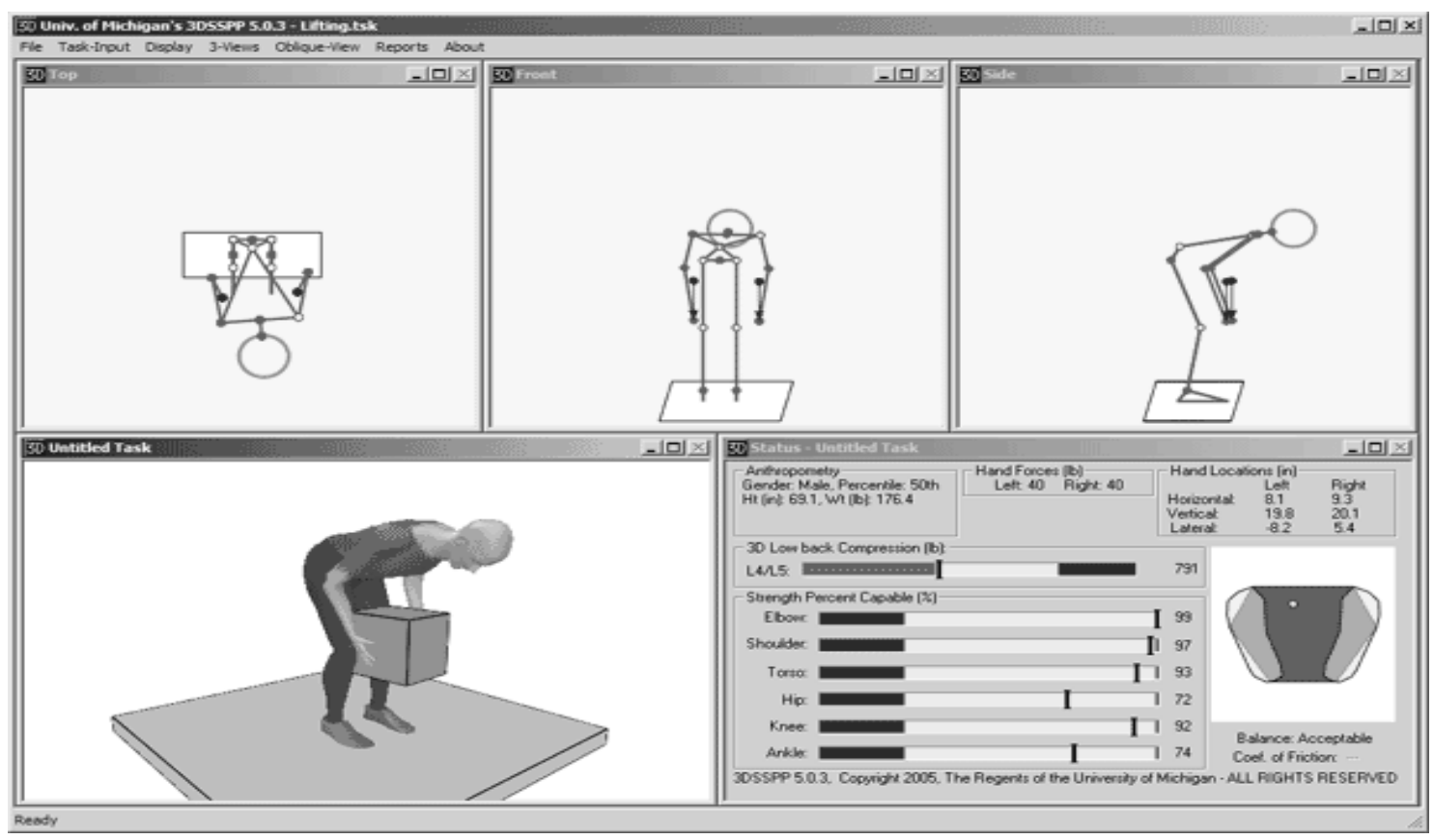

Figure 2.4: The screenshot of 3DSSPP window after the posture was entered (University of Michigan, 2011)

A limited number of studies have employed biomechanical models in an attempt to calculate forces on the lower lumbar spine or shoulder during pushing and pulling exertions (Knapik \& Marras, 2009). Resnick and Chaffin (1995) used such a single equivalent muscle model - a 2D dynamic biomechanical model developed by the University of Michigan to examine the pushing and pulling of carts weighing between 45-450 kg. The peak compression forces at the L5S1 spinal disc were computed using the sum of the dynamic external forces at the L5S1 disc and the pseudo-dynamic internal forces at the L5S1 disc based on the dynamic moment and static contractile forces on the erector spinae and rectus abdominal muscles. Calculated static compression forces at the L5S1 spinal disc were consistently above the NIOSH action limit of $3400 \mathrm{~N}$ (about 5000 N) for strong subjects when pushing carts over $225 \mathrm{~kg}$. Spine shear forces were not reported in the study. De Looze et al. (1995) employed a 2D dynamic model of the upper body with a single extensor muscle to evaluate pushing and pulling of three different refuse collecting methods. On the basis of movement analysis, force measurements and biomechanical modeling, spinal compression, and shear forces were estimated. The peak compression and shear force in tilting/pushing and pulling mini-containers ranged from 1657 to $2654 \mathrm{~N}$ and from 123 to $248 \mathrm{~N}$ respectively. Another study of refuse collectors 
employing a quasi-static 2D link segment model reported that the calculated compression forces at L4/L5 were rather small and ranged between 605-1445 $\mathrm{N}$ and the corresponding shear forces were below $202 \mathrm{~N}$ when transferring a load of $50 \mathrm{Kg}$ (Schibye, Søgaard, et al., 2001). In a similar type of study Laursen \& Schibye (2002) evaluated the effect of pushing and pulling a two-wheeled waste containers on different surfaces: flagstones, paving stones, and grass. It was reported that the lumbar spine compression force was below $1800 \mathrm{~N}$ and the shear force was below $200 \mathrm{~N}$ in all situations. The shoulder torque when pulling with one hand was up to $80 \mathrm{Nm}$. In a recent study of Jäger et al. (2007), a biomechanical model ("THE DORTMUNDER") was used quasi-statically for quantifying the mechanical load on the lumbar spine. However, this model has restrictions for use. For example, it was used quasi-statically so that inertial effects of body-mass acceleration on lumbar load were neglected.

In all the aforementioned studies, static/quasi-static 2D, single equivalent muscle models were employed. This type of biomechanical model would not be sensitive to the trunk muscle co-contraction expected during dynamic pushing and pulling activities (Lee, et al., 1989; Knapik \& Marras, 2009; Andres \& Chaffin, 1991). One of the significant simplifying assumptions inherent in these static models is that the coactivation of the trunk musculature during the dynamic $\mathrm{MMH}$ activities is negligible. The trunk is truly a multiple muscle system with many major muscle groups supporting and loading the spine. Studies have demonstrated that there is a significant coactivation occurring in many of the major trunk muscle groups during dynamic lifting activities. This coactivation is important because all the trunk muscles have the ability to load the spine. Coactivation of trunk muscles provides joint stiffening, stability and controlled movement; however, coactivation also substantially increases mechanical loads such as compression and shear forces on lumbar spine (Garg \& Kapellusch, 2009). Thus, assumptions regarding single equivalent muscles within the trunk can lead to erroneous conclusions about spine loading during a task. The study of Granata and Marras (1995a) reported that those biomechanical analyses that neglected muscle coactivity during dynamic lifting exertions might underestimate the spine compression force by as much as $45 \%$ and shear force by as much as $70 \%$. In an effort to more accurately estimate the 
loads on the lumbar spine, especially under complex, changing or dynamic exertions, multiple muscle systems models will be essential to assess the risk of injury.

\subsubsection{EMG-assisted multiple muscle system models}

In order to overcome such limitations as static or quasi-static mechanics, inaccurate prediction of muscle coactivity, neglecting the role of muscle co-contraction in quantifying spine loads, one of the few means to accurately account for the effect of the trunk muscle system coactivation upon spine loading is through the use of biologically assisted models. The most common of these models are dynamic and electromyography (EMG)-assisted models. These models take into account the individual recruitment patterns of the muscles during a specific lift for a specific individual. By directly monitoring muscle activity, the EMG-assisted model can determine individual muscle force and the subsequent spinal loading. These models have been developed and tested under bending and twisting dynamic motion conditions and have been validated in their ability to predict spine loads in three dimensions of loading during dynamic lifting activities (Marras \& Granata, 1997a, 1995; Granata \& Marras, 1995b; Marras \& Sommerich, 1991a, 1991b; Granata \& Marras, 1993).

The EMG-driven models "employ dynamic load in the hands, kinematic input, moment about the three axes of the low back normalized EMG, muscle-cross section area, a gain factor to represent muscle force per unit area and modulation factors describing EMG and force behavior as a function of muscle length and velocity to determine tensile load in each muscle" (Garg \& Kapellusch, 2009). Input data required by these EMG-driven models include time-domain EMG, exertion kinetics, and kinematics. Maximum exertion EMG levels and subject anthropometry were also employed to calibrate and format the dynamic data suitable for use in the model mechanics. The cross-sectional area of each muscle was computed from regression equations based on the subject's trunk depth and breadth. Trunk velocity was computed from dynamic measures of trunk flexion, twist, and lateral angles collected from a lumbar motion goniometer (lumbar motion monitor or LMM). Collected kinematic data were used in the model to: (1) describe the trunk motion as a function of time, (2) determine 
the muscle force and moment vector directions, and (3) modulate muscle EMG values to account for muscle length and velocity artifact. The time-domain myoelectric data collected from trunk muscles such as the right and left latissimus dorsi, erector spinae, rectus abdominus, internal abdominal obliques, and external abdominal obliques represent muscle activity and were used to calculate relative muscle force. An example of the EMG-assisted model is shown in Figure 2.5.

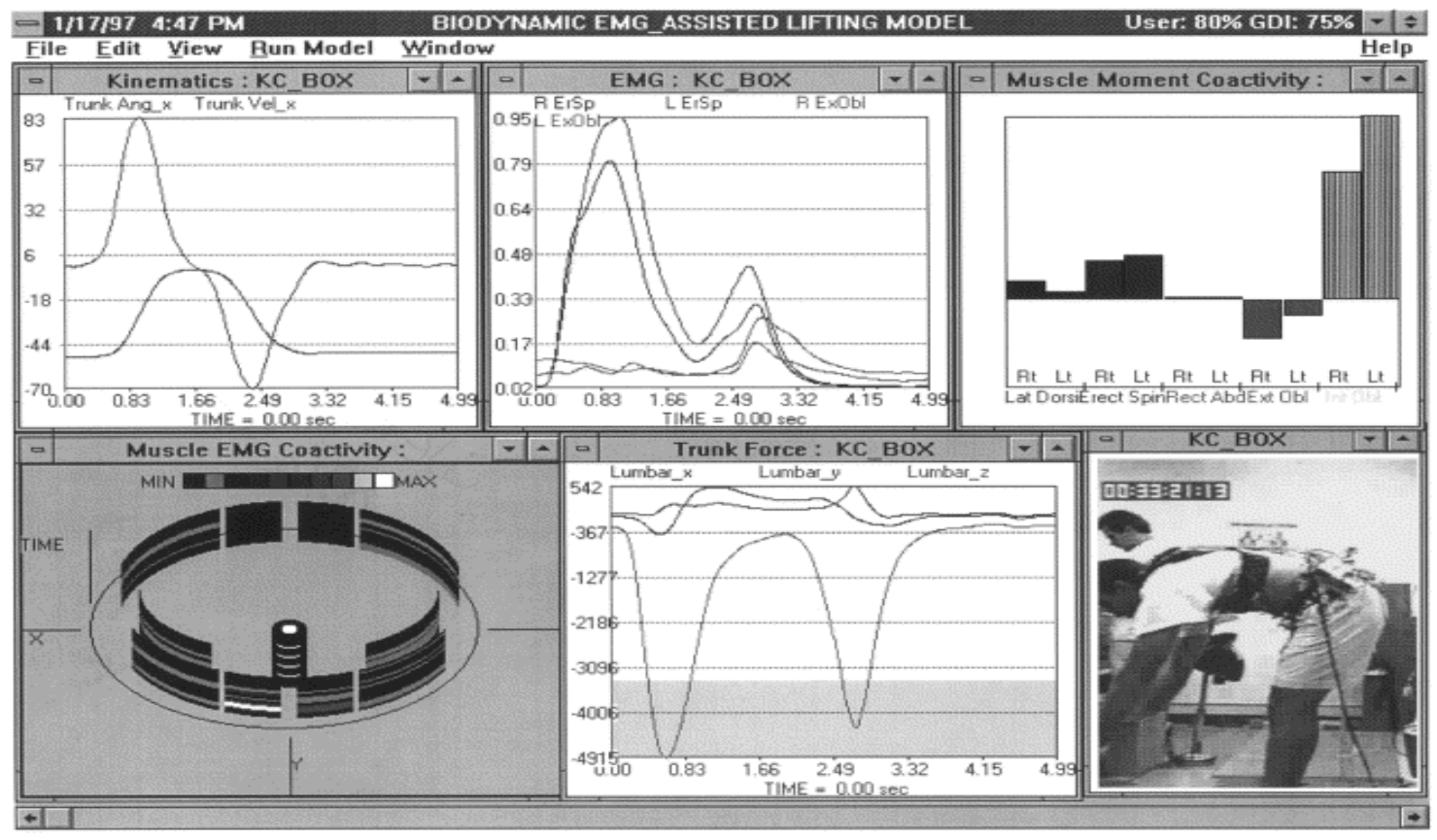

Figure 2.5: An example of the EMG-assisted model (Marras \& Granata, 1997b)

However, these EMG-driven biomechanical models have seldom been used to assess pushing and pulling because they have been optimized for back extension activities as opposed to the flexion and complex loading conditions expected during pushing and pulling (Knapik \& Marras, 2009). Knapik and Marras (2009) demonstrated that it is possible to assess risk associated with pushing and pulling activities by employing an EMG-assisted model to account for trunk muscle coactivation in assessing spine loading at the various lumbar spine levels. The model used kinematic information about the trunk along with EMG information from the trunk musculature to estimate spinal loads, as well as predict the moments imposed on the spine in 3D space. Another study of Hoozemans et al. (2004) used a different EMG-driven biomechanical model (van Dieen, 1997; van Dieen \& Kingma, 1999) to estimate compression and shear forces 
at the L5S1 intervertebral disc. However, this study focused primarily upon the portion of the exertion (e.g., initial effort vs. sustained exertion) that yielded the largest loads on the spine (Knapik \& Marras, 2009).

One limitation of such EMG-assisted models is that they require significant instrumentation on the worker, such as the Lumbar Motion Monitor (LMM) and several pairs of $\mathrm{Ag} / \mathrm{AgCl}$ surface electrodes attached to the involved trunk muscles. These instrumentations may restrict human movement. Another limitation of such models is that it is not a comprehensive full-body musculoskeletal model. Such EMG-driven models are limited to assessing the loading on the lumbar spine. The loading of various other major body joints and segments cannot be explored by these models. 


\section{CHAPTER 3: RESEARCH RATIONALE}

\subsection{Problem Statement}

With the advancement in the field of e-commerce in recent years, most manufacturing is done offshore, and there has been an increase in the number of distribution centers in the United States. At the distribution centers, workers primarily receive and transfer goods, and this has changed the way physical forces are being applied at the workplace. In the United States, nearly half of $\mathrm{MMH}$ activities common at workplaces demand pushing and pulling exertions (Hoozemans, et al., 2002b). In many other instances, pushing/pulling loads using assistive devices or interfaces such as trolleys, carts, trucks, wheelbarrows, etc., are the intervention strategies used to prevent lifting and carrying loads to reduce excessive loading of the lumbosacral (L5S1) joint, advocated as the weakest link in the vertebral column. Thus, pushing/pulling exertions are becoming increasingly predominant at various workplaces. Some epidemiological studies suggest that work-related pushing/pulling exertions are associated with musculoskeletal disorders of the low back (Hoozemans, et al., 1998; van der Beek, et al., 1993; Ozguler, et al., 2000; Klein, et al., 1984; Harber, et al., 1987; Garg \& Moore, 1992; Fuortes, et al., 1994; Frymoyer, et al., 1980; Damkot, et al., 1984; NIOSH, 1997, 1981), shoulder, and other upper extremities (Hoozemans, et al., 1998; van der Beek, et al., 1993; Smedley, et al., 2003; Hoozemans, et al., 2002a, 2002b; Hughes, et al., 1997). Although epidemiological studies provide important indications as to the causality of the association, experimental studies are essential to estimate the quantitative information required to understand the process of how exposure may lead to musculoskeletal disorders (Hoozemans, et al., 2004; Keyserling, 2000).

Our literature review of experimental studies that evaluate pushing/pulling exertions identified the following research gaps:

1) Early studies that evaluated musculoskeletal loading primarily focused on static exertions. Recent dynamic pushing/pulling exertions were evaluated to examine changes in the hand forces or physiological parameters, such as heart rate and oxygen uptake or 2D low back and shoulder loading. A variety of studies in this field have investigated hand force exertion with particular concern toward the three motion phases (initial, sustained, and ending phases). 
2) Past research studies have employed biomechanical models, such as single equivalent muscle models (Schibye, Søgaard, et al., 2001; Resnick \& Chaffin, 1995; Laursen \& Schibye, 2002; De Looze, et al., 1995) and EMG-assisted biomechanical models (Hoozemans, et al., 2004; Knapik \& Marras, 2009) to quantify the loading of the lumbar spine during pushing and pulling. The 3D loading of the various other major body joints and segments using a more comprehensive full-body musculoskeletal model has not been explored.

3) Load weight is the most frequently studied task parameter during pushing/pulling exertions due to the strong linear relationship between biomechanical loading and load weight (Glitsch, et al., 2007; Jäger, et al., 2007; Hoozemans, et al., 2004; Laursen \& Schibye, 2002). Limited research is available regarding pushing/pulling along ramps despite the prevalence of such tasks at workplaces.

4) Furthermore, past studies did not look at the changes in the biomechanical demands associated with uphill pushing and downhill pulling exertions along graded walkway.

The current study was aimed to quantify musculoskeletal loading during dynamic pushing and pulling exertions under varying task demands. The effect of three task parameters: load weight, walkway gradient, and direction of force exertion on the three-dimensional (3D) musculoskeletal loading of the lumbosacral (L5S1) joint of the low back and three major shoulder joints (sternoclavicular, acromioclavicular, and glenohumeral) were studied using a full-body biomechanical modeling technique.

\subsection{Objectives}

The purpose of this study, quantification of musculoskeletal loading associated with dynamic pushing and pulling exertions under varying task demands, would be achieved through the following objectives:

Objective 1: evaluation of the effect of load weight transferred during pushing/pulling exertions on the 3D loading of the low back and shoulder joints

This objective would be achieved by studying pushing and pulling tasks performed using a most commonly used two-wheeled cart under three weight conditions: 20,30 , and $40 \mathrm{~kg}$, in the 
laboratory setting. These weights represent the loads displaced with the cart during material handling activities that are common at various workplaces.

Objective 2: evaluation of the effect of walkway gradient during pushing/pulling exertions on the 3D loading of the low back and shoulder joints

This objective would be achieved by studying pushing/pulling along a flat, $5^{\circ}$, and $10^{\circ}$ ramped walkway, in the laboratory setting. The gradients represent the ramps that are normal at various workplaces.

Objective 3: evaluation of the effect of the direction of force exertion along ramps during pushing/pulling exertions on the 3D loading of the low back and shoulder joints

This objective would be achieved by studying pushing/pulling along the ramps, uphill and downhill, respectively. The cart was pushed or pulled in front of the human body.

\subsection{Research Hypotheses}

The role played by the load weight, walkway gradient, and direction of force exertion during dynamic cart pushing and pulling tasks were evaluated by testing five hypotheses. The hypothesis statements are as follows:

\subsubsection{Hypothesis 1: Impact of load weight}

The musculoskeletal loading of major low back and shoulder joints would increase, corresponding to the increase in the load weight displaced by the cart. The joint loading during displacing the load weight of 20,30, and $40 \mathrm{~kg}$ along the same walkway was used to test this hypothesis.

\subsubsection{Hypothesis 2: Impact of walkway gradient}

The musculoskeletal loading of major low back and shoulder joints would increase, corresponding to the increase in the gradients when displacing the cart along the walkway. The joint loading at the same weight condition, during cart pushing over flat $\left(0^{\circ}\right)$ and two ramped $\left(5^{\circ}\right.$ and $10^{\circ}$ ) walkways and during cart pulling over the two ramped $\left(5^{\circ}\right.$ and $\left.10^{\circ}\right)$ walkways was used to test this hypothesis. 


\subsubsection{Hypothesis 3: Impact of direction of force application along the ramp}

The musculoskeletal loading of major low back and shoulder joints would be higher during downhill pulling than during uphill pushing along the ramps. The joint loading during cart uphill pushing and downhill pulling tasks along the ramps of $5^{\circ}$ and $10^{\circ}$ while moving the same load was used to test this hypothesis.

\subsubsection{Hypothesis 4: Low back loading in anterior-posterior direction}

The low back loading at the L5S1 joint level in the anterior-posterior (AP) direction would be significantly affected by the pushing/pulling exertions evaluated in this study. . At the extreme task conditions (higher ramp, higher weight) the loading of the L5S1 joint in the anterior-posterior (AP) direction may exceed the recommended tolerance limit of this joint The L5S1 joint loading in AP direction during cart uphill pushing and downhill pulling tasks along the ramps of $5^{\circ}$ and $10^{\circ}$ while moving the same load was used to test this hypothesis.

\subsubsection{Hypothesis 5: Shoulder loading at acromioclavicular joint}

The musculoskeletal loading at the acromioclavicular joint would be significantly affected by the weight, gradient, and direction of force application during the pushing and pulling tasks. The overall loading of this joint would be comparable with the loading of the glenohumeral joint during the pushing/pulling exertions evaluated in this study. 


\section{CHAPTER 4: METHODS}

\subsection{Approach}

The effect of dynamic pushing/pulling tasks on musculoskeletal loading was examined by performing a laboratory-based study. Human participants performed cart pushing/pulling tasks on a flat and two inclined walkways under three weight conditions: 20,30 , and $40 \mathrm{~kg}$. Whole body 3-D joint kinematics and ground reaction force data were recorded using an eightcamera marker-based optical motion analysis system coupled with two ground reaction force

platforms. The experimental data were used for running a full-body musculoskeletal model to compute the biomechanical loading of the low back at the L5S1 level and three shoulder joints (sternoclavicular, acromioclavicular, and glenohumeral).

\subsection{Participants}

In order to control the confounding effect of the gender factor, only male participants were recruited in the study. In addition, men are more likely to be exposed to manual material handling tasks in real workplaces. Other pushing and pulling studies that used only male subjects include Bennett et al. (2008), De Looze et al. (2000), Hoozemans et al. (2004), Kuijer et al. (2002), Laursen and Schibye (2002), Lett and McGill (2006), and Schibye et al. (2001).

Twelve male participants with no history of musculoskeletal abnormalities participated in this study. The physical Activity Readiness Questionnaire (PAR-Q, British Columbia Ministry of Health) was used to screen participants for cardiac and other health problems (e.g., dizziness, chest pain, heart trouble) (Appendix A). Participants who answered yes to any of the questions on the PAR-Q were excluded. Prior to data collection, the experimental procedures and the demands of testing were explained to the participants, and their signatures were obtained on the informed consent form approved by the Institute Review Board at West Virginia University (Appendix B).

\subsection{Experimental Design}

This study consisted of two experimental sessions. In session one, the effect of load weight on biomechanical loading of low back and shoulder joints was evaluated. In session two, 
the effects of load weight, walkway gradient, and direction of force application on biomechanical loading of the low back and shoulder joints were evaluated. A $3 \times 2 \times 2$ full factorial experimental design was used. During both sessions, three trials of each experimental condition were recorded and the trial order was randomized.

\subsubsection{Independent variables}

In session one, participants performed cart pushing on a flat walkway. The load weight and the individual participants (treated as a block) were the independent variables. The load weight had three fixed levels: "light" load weight of $20 \mathrm{~kg}$, a "medium" load weight of $30 \mathrm{~kg}$, and a "heavy" load weight of $40 \mathrm{~kg}$.

In session two, participants performed uphill pushing or downhill pulling tasks at three weight conditions along two gradients of walkway. The independent variables were: load weight, walkway gradient, and direction of force application. The levels of load weight were the same as in session one. Walkway gradient had two fixed levels: $5^{\circ}$ and $10^{\circ}$, and direction of force application also had two fixed levels: uphill pushing or downhill pulling.

\subsubsection{Dependent variables}

Low Back Loading at the L5S1 joint: The lumbar spine is often a site of pain associated with forceful work activities. The L5S1 joint of the spine was chosen as the analysis of low back loading, because it is the lumbar joint that is expected to undergo the most stress (Kingma \& van Dieen, 2004). The peak reaction forces acting at this joint in three anatomical directions: compression (proximal-distal), anterior-posterior (AP) shear, and medio-lateral (ML) shear were treated as the dependent variables for low-back loading. The abbreviations used for these dependent variables are as follows:

L5S1MedioLateral Force - L5S1-ML

L5S1Proximal-Distal Force - L5S1-PD

L5S1Anterior-Posterior Force - L5S1-AP

Shoulder Joint Loading: The shoulder complex consists of three joints: (1) sternoclavicular joint (SC); (2) acromioclavicular joint (AC); and (3) glenohumeral joint (GH). A brief anatomical description of these joints is provided in the literature review chapter (see 
Section 2.3). The common work-related pathologies associated with the shoulder complex include: shoulder tendinitis, rotator cuff pain, and nonspecific shoulder pain. Repetitive loading of shoulder joints has been identified as one of the causal factors for these shoulder complex pain syndromes (NIOSH, 1997). Therefore, loading of the shoulder joint was assessed by using peak reaction forces acting in three anatomical directions at sternoclavicular joint, acromioclavicular, and glenohumeral joints. Table 4.1 lists the dependent variables used for studying shoulder joint loading.

\subsubsection{Statistical models}

The current study was divided into two sessions: (1) the load weight was evaluated for whether or not the increases of the weight transferred $(20,30$, and $40 \mathrm{~kg}$ ) by the cart increases participants' low-back and shoulder joint loading when they pushed the cart along a horizontal walkway; (2) the load weight, walkway gradient, and direction of force application were evaluated for whether or not the increases of the weight transferred $(20,30$, and $40 \mathrm{~kg}$ ), the gradient of the ramp $\left(5^{\circ}\right.$ and $\left.10^{\circ}\right)$, and whether the changes of direction of force exertion along the ramps (uphill pushing and downhill pulling) increased participants' low-back and shoulder joint loading when they pushed/pulled the cart along ramps. The two sessions were evaluated respectively, and two statistical models were built as below:

\section{Model 1 for session one along the flat walkway:}

$$
y F_{i l}=\mu+\alpha_{i}+\gamma_{l}+\epsilon_{i l} \quad\left\{\begin{array}{l}
i=1, \ldots, a \\
l=1, \ldots, n
\end{array}\right.
$$

Where,

$y F$ represents the low back and shoulder joints loading for pushing exertions along the flat walkway.

$\mu$ is the overall mean common to all observations.

$\alpha_{i}$ is the effect of load weight transferred by the cart pushed by the subjects, $i=1,2,3$.

$\gamma_{l}$ is the effect of subjects, $l=1,2, \ldots, 12$.

$\epsilon_{i l}$ is a random error term. 
Table 4.1: Dependent variables used for studying shoulder joint loading

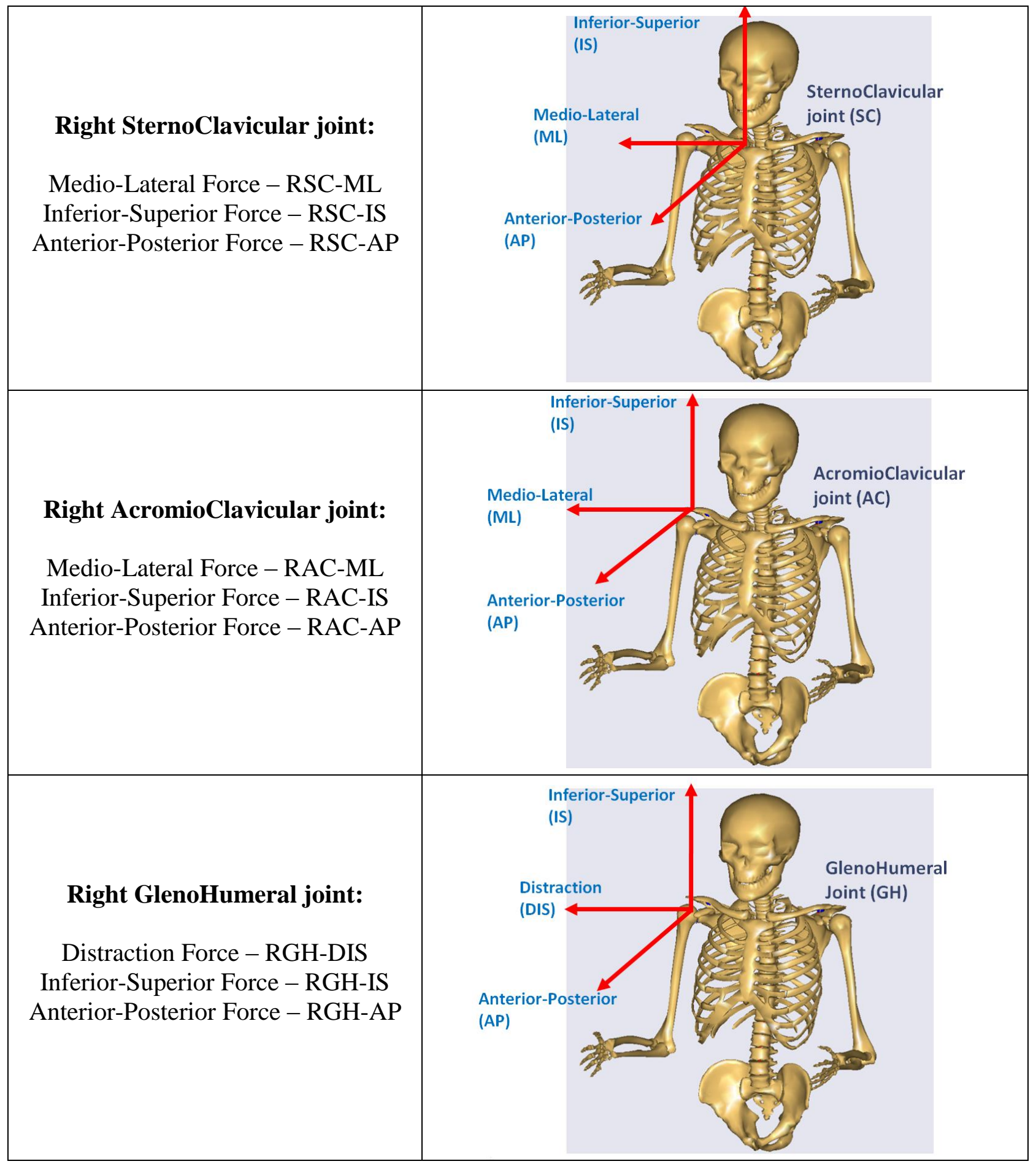




\section{Model 2 for session two along ramps:}

$y R_{i j k l}=\mu+\alpha_{i}+\beta_{j}+\tau_{k}+\gamma_{l}+(\alpha \beta)_{i j}+(\alpha \tau)_{i k}+(\beta \tau)_{j k}+(\alpha \beta \tau)_{i j k}+\epsilon_{i j k l}\left\{\begin{array}{l}i=1, \ldots, a \\ j=1, \ldots, b \\ k=1, \ldots, c \\ l=1, \ldots, n\end{array}\right.$

Where,

$y R$ represents the low back and shoulder joint loading during cart pushing and pulling exertion along the ramped walkways.

$\mu$ is the overall mean common to all observations.

$\alpha_{i}$ is the effect of weight transferred by the cart pushed by the subjects, $i=1,2,3$.

$\beta_{j}$ is the effect of walkway gradient, $j=1,2$.

$\tau_{k}$ is the effect of direction of force application along ramps, $k=1,2$.

$\gamma_{l}$ is the effect of subjects, $l=1,2, \ldots, 12$.

$(\alpha \beta)_{i j}$ is the interaction effect of weight transferred by the cart and walkway gradient.

$(\alpha \tau)_{i k}$ is the interaction effect of weight transferred by the cart and direction of force application along ramps.

$(\beta \tau)_{j k}$ is the interaction effect of walkway gradient and direction of force application along ramps.

$(\alpha \beta \tau)_{i j k}$ is the interaction effect of the three factors: weight transferred by the cart, walkway gradient, and direction of force application along ramps.

$\epsilon_{i j k l}$ is a random error term. 
In the first model, the load weight transferred $\left(\alpha_{i}\right)$ was treated as a fixed factor. It was assumed that this factor had no effect on the 3D loading of low back and shoulder joints during the cart pushing tasks along the flat walkway. That is:

$$
\sum_{i=1}^{a} \propto_{i}=0
$$

Subjects $\left(\gamma_{l}\right)$ were treated as a random factor, and it was assumed that it is NID $\left(0, \sigma_{\gamma}{ }^{2}\right)$ random variable. Random error $\epsilon_{i l}$ followed NID $\left(0, \sigma^{2}\right)$.

One table of analysis of variance (ANOVA) was provided for each response variable in this model (Appendix I). Therefore, the appropriate F tests were applied to test the means of the fixed factor, i.e., effects of the load weight transferred by the cart are equal, such as:

$H_{0}: \alpha_{1}=\alpha_{2}=\alpha_{3}=0$

$H_{1}$ : at least one $\alpha_{i} \neq 0$

In this model, the Type I error $\alpha=0.05$ and Power of the test (1- $\beta$ ), which equals 0.90 , were chosen for the hypothesis test and sample size determination in Section 4.3.4.

In the second model, the load weight transferred $\left(\alpha_{i}\right)$, walkway gradient $\left(\beta_{j}\right)$, and direction of force application along ramps $\left(\tau_{k}\right)$ were treated as fixed factors. It was assumed that each factor and the two-way or three-way interaction factors had no effect on the 3D loading of low back and shoulder joints during the uphill pushing or downhill pulling tasks along the ramps. That is:

$$
\begin{gathered}
\sum_{i=1}^{a} \alpha_{i}=0, \quad \sum_{j=1}^{b} \beta_{j}=0, \quad \sum_{k=1}^{c} \tau_{k}=0, \\
\sum_{i=1}^{a} \sum_{j=1}^{b}(\alpha \beta)_{i j}=0, \quad \sum_{i=1}^{a} \sum_{k=1}^{c}(\alpha \tau)_{i k}=0, \quad \sum_{j=1}^{b} \sum_{k=1}^{c}(\beta \tau)_{j k}=0,
\end{gathered}
$$

and

$$
\sum_{i=1}^{a} \sum_{j=1}^{b} \sum_{k=1}^{c}(\alpha \beta \tau)_{i j k}=0
$$

Subjects $\left(\gamma_{1}\right)$ were treated as a random factor, and it was assumed that it is $\operatorname{NID}\left(0, \sigma_{\gamma}{ }^{2}\right)$ random variable. Random error $\epsilon_{\mathrm{il}}$ and $\epsilon_{\mathrm{ijkl}}$ followed $\operatorname{NID}\left(0, \sigma^{2}\right)$. 
ANOVA tables were provided for each response variable in this model (Appendix $\mathrm{J}$ ). Therefore, the appropriate $\mathrm{F}$ tests were applied on testing the means of the fixed factor effects are equal, such as

$$
\begin{aligned}
& H_{0}: \alpha_{\mathrm{i}}=0, \beta_{\mathrm{j}}=0, \tau_{\mathrm{k}}=0 \text { and } \\
& \quad(\alpha \beta)_{\mathrm{ij}}=0,(\alpha \tau)_{\mathrm{ik}}=0,(\beta \tau)_{\mathrm{jk}}=0 \text {, and }(\alpha \beta \tau)_{\mathrm{ijk}}=0, \\
& H_{1} \text { : at least one } \alpha_{\mathrm{i}} \neq 0, \beta_{\mathrm{j}} \neq 0 \tau_{\mathrm{k}} \neq 0 \text { and } \\
& \quad \text { at least one }(\alpha \beta)_{\mathrm{ij}} \neq 0,(\alpha \tau)_{\mathrm{ik}} \neq 0,(\beta \tau)_{\mathrm{jk}} \neq 0 \text {, and }(\alpha \beta \tau)_{\mathrm{ijk}} \neq 0,
\end{aligned}
$$

In this model, the Type I error $\alpha=0.05$ and Power of the test (1- $\beta$ ), which equals 0.90 , were chosen for the hypothesis test and sample size determination in 4.3.4.

\subsubsection{Power analysis and sample size determination}

Operating characteristics curves (OC curves), a graph of $\beta$ (type II error probability) versus the true difference in means, play an important role in the choice of sample size in experimental design problems. Therefore, the OC curves were used to do a power analysis and to determine the number of subjects to be recruited in this research.

The random factor subject $\left(\gamma_{1}\right)$ was treated as a block, so determining the number of subjects was actually calculating the number of blocks. For both Model 1 and Model 2, the OC curves were used with the formula:

$$
\lambda=\sqrt{1+\frac{c \sigma_{\gamma}^{2}}{\sigma^{2}}}
$$

where,

$$
\sigma_{\gamma}^{2}=\mathrm{MSBL}, \sigma^{2}=\mathrm{MSE} .
$$

History data were collected from five subjects $(c=5)$ performing both sessions. MSBL and MSE were calculated. Appendix C shows more detailed calculations of the sample size required for both parts of the study to achieve the required power of at least 0.90 .

For the first model, by using OC curves, it was found that $\beta \approx 0.5$. Therefore, the power of the test was approximately $(1-\beta)=1-0.5=0.5$, which is less than the pre-selected power of at least 0.90 . Table 4.2 shows the power for different number of subjects for Model 1 (pushing along the flat walkway). Note that eight subjects give a $\beta$ risk of about 0.1 , or approximately a 
90\% chance of rejecting the null hypothesis, and we can conclude that the load weight transferred by the cart $(20,30$ and $40 \mathrm{~kg})$ had an effect on the low back and shoulder joints.

Table 4.2: Power values for different number of subjects for Model 1

\begin{tabular}{|c|c|c|c|}
\hline $\mathbf{c}$ & $\boldsymbol{\lambda}$ & $\boldsymbol{\beta}$ & Power $(\mathbf{1 - \beta})$ \\
\hline 5 & 2.05 & 0.5 & 0.5 \\
\hline 6 & 2.2 & 0.34 & 0.66 \\
\hline 7 & 2.34 & 0.2 & 0.8 \\
\hline 8 & 2.48 & 0.1 & 0.9 \\
\hline
\end{tabular}

Proceeding in a similar manner, for the second model, it was found that $\beta \approx 0.57$. Therefore, the power of the test was approximately $(1-\beta)=1-0.57=0.43$, which is also less than the pre-selected power of at least 0.90 . Table 4.3 shows the power for a different number of subjects for Model 2 (uphill pushing and downhill pulling along the ramp). Note that eight subjects give a $\beta$ risk of about 0.1 , or approximately a $90 \%$ chance of rejecting the null hypothesis, and we can conclude that the factors considered in this part of the study, such as load weight transferred by the cart $(20,30$ and $40 \mathrm{~kg})$, walkway gradient $\left(5^{\circ}\right.$ and $\left.10^{\circ}\right)$, and direction of force application along the ramps (uphill pushing and downhill pulling), had main effects on the low back and shoulder joints.

Table 4.3: Power values for different number of subjects for Model 2

\begin{tabular}{|c|c|c|c|}
\hline $\mathbf{c}$ & $\boldsymbol{\lambda}$ & $\boldsymbol{\beta}$ & Power (1- $\boldsymbol{\beta})$ \\
\hline 5 & 1.76 & 0.57 & 0.43 \\
\hline 6 & 1.87 & 0.4 & 0.6 \\
\hline 7 & 1.98 & 0.19 & 0.81 \\
\hline 8 & 2.08 & 0.1 & 0.9 \\
\hline
\end{tabular}

Therefore, eight subjects would be sufficient for both sessions of the current study to insure both power requests for the two models. However, we slightly increased the sample size to twelve subjects to guard against the possibility that the prior estimate of the standard deviation was too conservative. 


\subsection{Equipment}

In this study, the following pieces of equipment (Figure 4.1) were used for data collection, processing, and analysis: (1) a hand truck and three loads, (2) a wooden walkway, (3) a scissor lift table, (4) four bottle jacks, (5) two force plates, (6) an eight-camera optical motion capture system, and (7) a musculoskeletal modeling system.

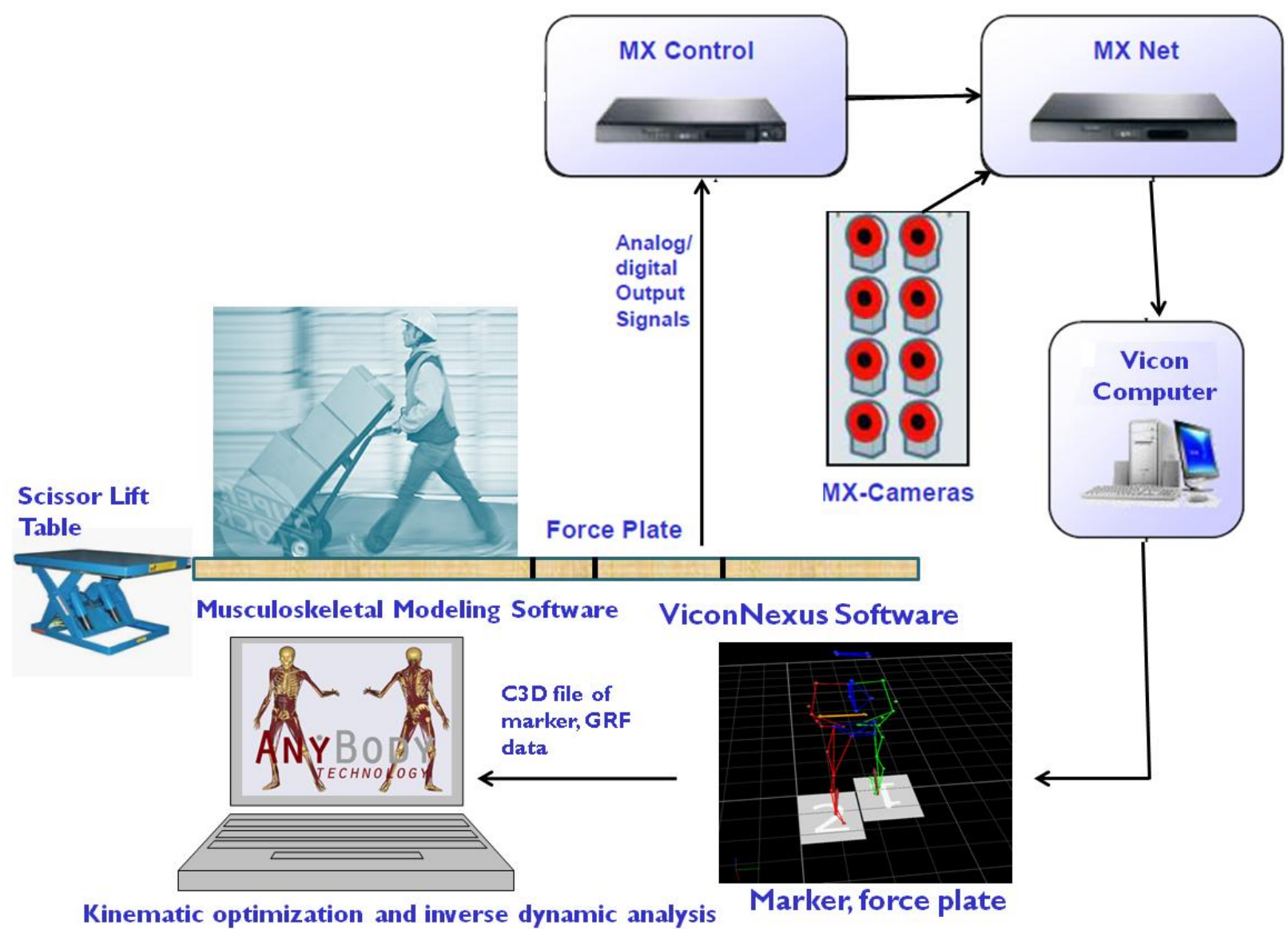

Figure 4.1: Equipment used for data collection, processing and analysis

\subsubsection{Hand truck and loads}

A two-wheeled Harper hand truck (CSLEDTK1935P, Harper Trucks, Inc., Wichita, KS, USA) (Figure 4.2) was used in this study. This is a standard, easily available and most commonly used hand truck in industrial settings (Keyserling, et al., 1999). This hand truck $(1.25 \mathrm{~m}$ height $\times 0.31 \mathrm{~m}$ depth $\times 0.33 \mathrm{~m}$ width) weighs $14.3 \mathrm{~kg}$ and has a capacity of $700 \mathrm{lbs}$. The height of the handle is $1.25 \mathrm{~m}$ above ground level with the cart in an upright position and about 
$0.85 \mathrm{~m}$ in a tilted position ready to be moved. Each wheel, made of rubber, is a 10-inch pneumatic tire. In the current study, the coefficient of friction for rubber wheels on a wooden walkway was estimated to be approximately 1.3 .

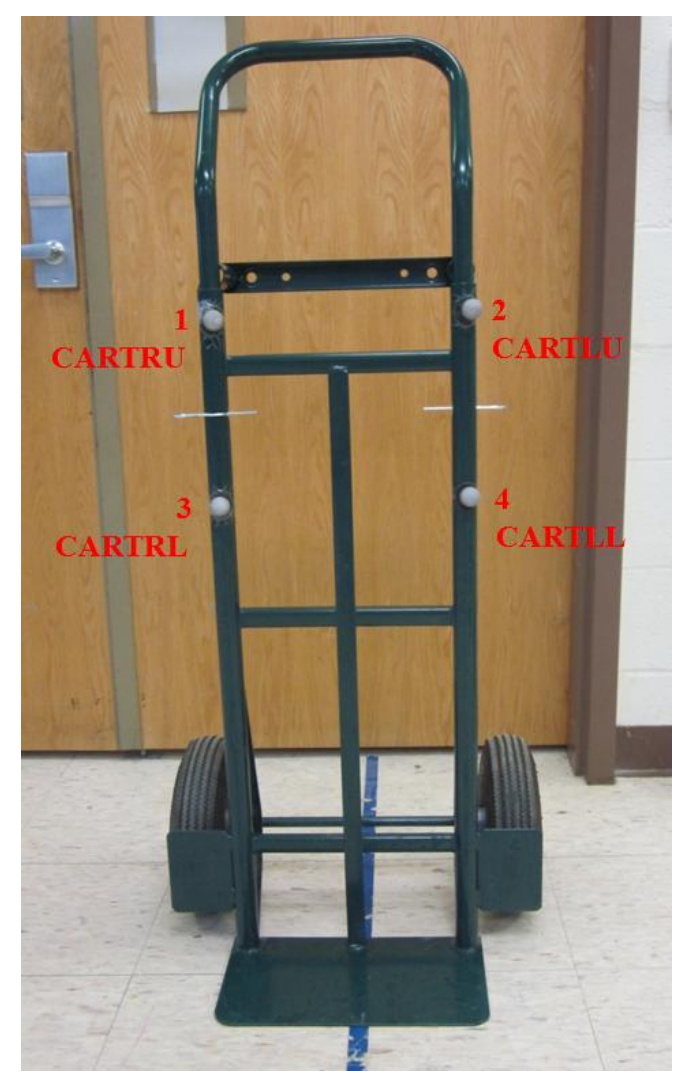

Figure 4.2: Hand truck used in this study attached with four reflective markers

\subsubsection{Wooden walkway, scissor lift table and bottle jacks}

A flat walkway (Figure 4.3 (a)) measuring 3 feet (wide) $\times 12$ feet (length) was created using a reinforced wooden frame with cut-outs to accommodate the force plates (Figure 4.5). A hydraulic scissor lift table (EHLT-4872-3-43, Vestil Manufacturing Corp., IN, USA) (Figure 4.4 (a)) was attached to one end of the wooden walkway using a hinge assembly. This table has a collapsed height of 7" and can be raised to a maximum height of 36." When completely collapsed, it flushes with the walkway providing a flat walking surface. To set up the $5^{\circ}$ (Figure 4.3 (b)) and $10^{\circ}$ (Figure 4.3 (c)) ramped walkways, the table height was raised to $12.6^{\prime \prime}$ and $25^{\prime \prime}$ using a hydraulic actuator. The capacity of the lift table was 3,000 lbs, which is sufficient to support the weight of the wooden walkway and the participant performing the dynamic cart 
pushing or pulling. In addition, the $48 "$ (width) $\times 72 "$ (length) top surface of the scissor lift table provided enough space for the participant to rest between trials.

While the ramp is in use, it is critical that it maintains both its strength and structural integrity. This is not only of paramount importance for safety, but also it is crucial so that the participant does not sense any anxiety-provoking motion in the ramp. If the participants are not comfortable walking on the ramp, they may be apprehensive and walk with an unnatural gait while pushing/pulling the cart. This may affect the quality of the ground reaction force (GRF) data. To prevent all these issues, the structure of the frame was designed to be stiff and rigid. Furthermore, four hydraulic bottle jacks (Torin Jacks, Inc., Ontario, CA) shown in Figure 4.4 (b) were used to support the walkway in the ramped configurations to improve its structural rigidity. The bottle jacks used in this study had a capacity of six tons and two of them were placed under each side of the inclined walkway close to the area where force plates were mounted.

\subsubsection{Force plates}

Two Kistler force plates (type 9286, Kistler Instrument Corp., Amherst, NY, USA) configured with the optical motion system were used to record the ground reaction force (GRF) (Figure 4.5) (Fischer, Kirchberg, \& Moessner, 2009; You, et al., 2001; Janz, et al., 2003; Desailly, et al., 2009). Each plate, made of aluminum, weighs about $18 \mathrm{~kg}$, measuring $60 \mathrm{~cm}$ (length) $\times 40 \mathrm{~cm}$ (width) $\times 3.5 \mathrm{~cm}$ (height). The force plate sensors use strain gauges to measure the three components of force in the $\mathrm{x}, \mathrm{y}$, and $\mathrm{z}$ and the corresponding moments around three orthogonal axes. The GRF data, collected at a frequency of $100 \mathrm{~Hz}$, were synchronized with the kinematic data recorded using the optical motion analysis system. The surface of the force plates was flushed with the walkway to accurately measure GRF as the participant makes contact with the force plate. Figure 4.5 shows the actual placement of the two force plates in the walkway. The two force plates were mounted in the walkway with an offset of $20 \mathrm{~cm}$ to allow acquisition of GRF of right and left foot steps during normal gait. In addition, this offset of $20 \mathrm{~cm}$ also allowed recognition of footsteps independent of force vectors exerted by the cart, which is essential for accurately running the biomechanical modeling analysis. 

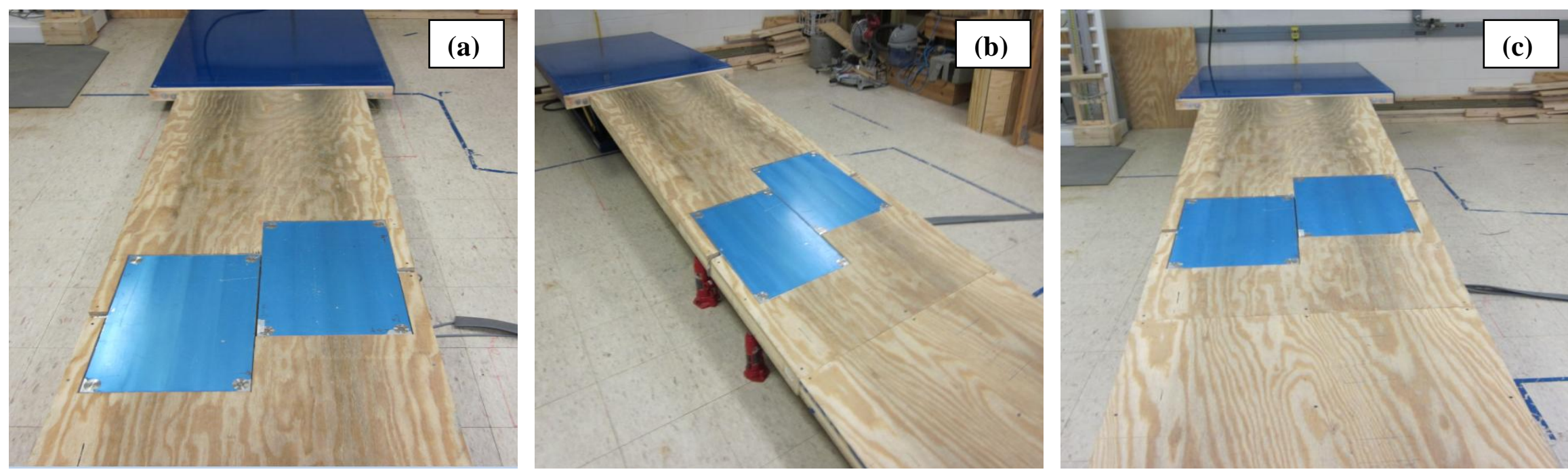

Figure 4.3: Wooden walkway: (a) flat; (b) $5^{\circ}$ ramp; (c) $10^{\circ}$ ramp
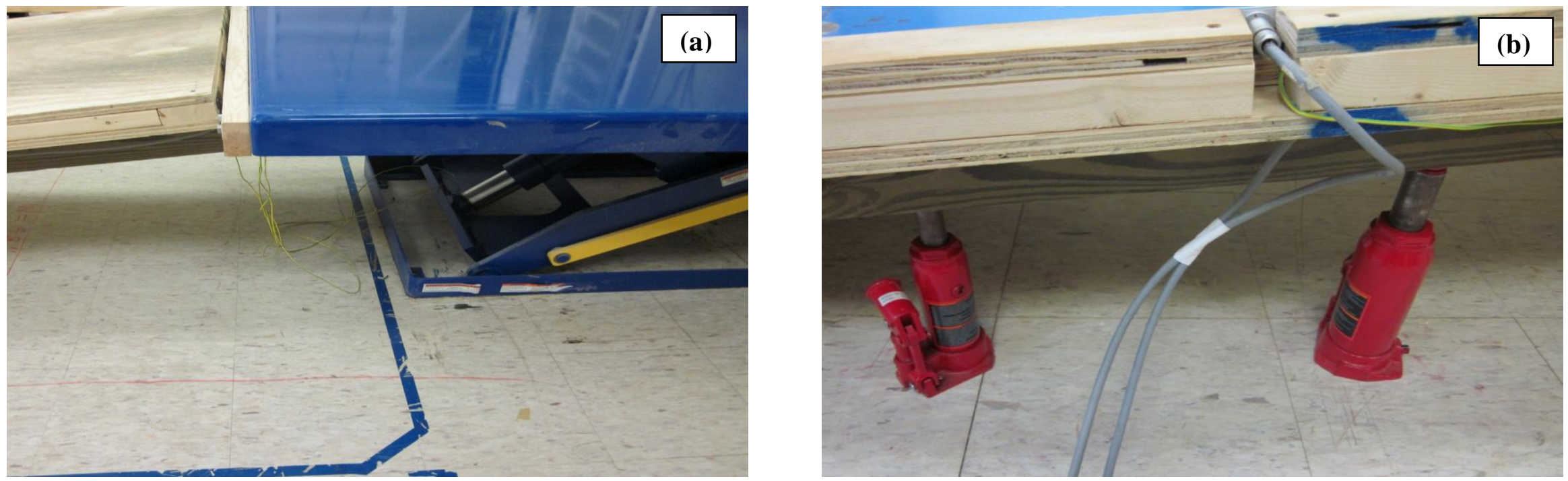

Figure 4.4: (a) Scissor lift table; (b) hydraulic bottle jacks to minimize the GRF data noise 


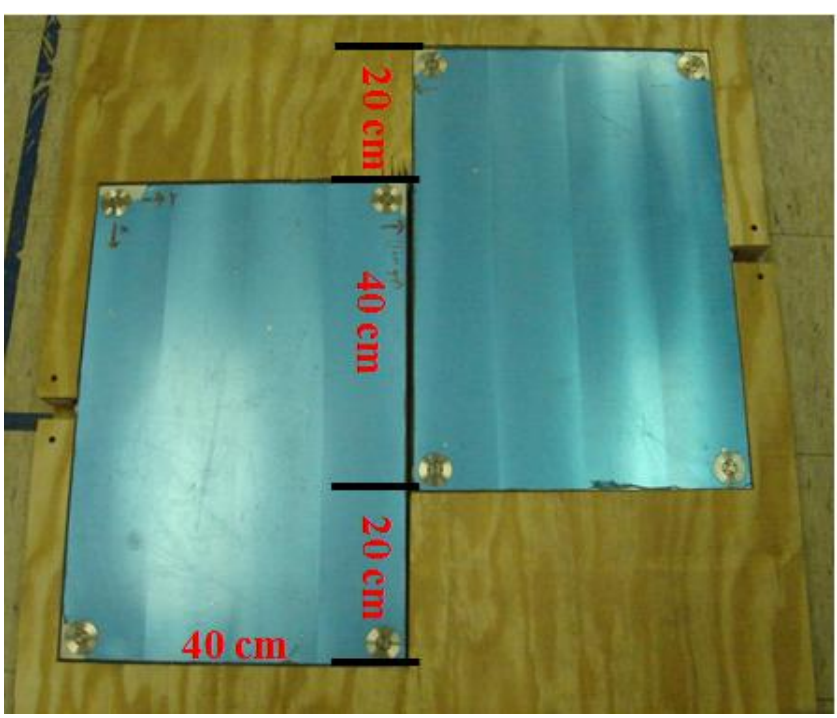

Figure 4.5: Two force plates embedded in wooden platform

\subsubsection{Optical motion capture system}
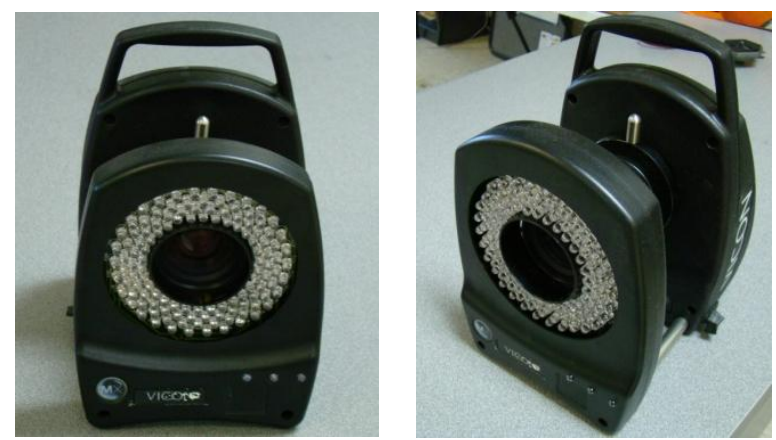

Figure 4.6: Pictures of a Vicon MX camera

This is an eight-camera optical motion analysis system (Vicon Oxford Metrics, UK). Figure 4.6 shows the front and side views of a Vicon MX camera. Each camera records 3D motion trajectories of the small reflective balls (markers) attached to the participants, at a frequency of $100 \mathrm{~Hz}$.

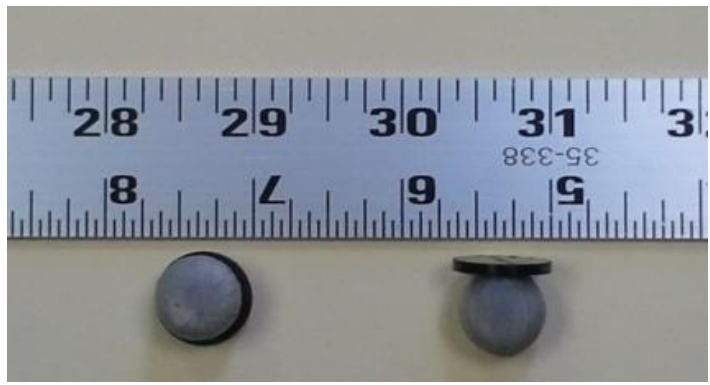

Figure 4.7: $14 \mathrm{~mm}(0.55 \mathrm{inch})$ reflective markers 
Light emitted from infrared sources mounted near the cameras is reflected off the markers and captured by the cameras. The markers are lightweight and interfere minimally with the subject's movements (Figure 4.7). The eight video cameras were placed around the walkway to create a capture volume. Each marker is simultaneously imaged by multiple cameras and the location of the marker in the 3D space ( $\mathrm{x}, \mathrm{y}, \mathrm{z}$ coordinates) is computed using triangulation by the Vicon Nexus software.

A Plug-in Gait (PIG) marker set was used for the kinematic data collection in this study. PIG is the Vicon implementation of the Conventional Gait Model which is widely used by the gait analysis community and has been validated in a number of studies (Andreoni, et al., 2009; Hwang \& Kim, 2009; Riley, et al., 2007; Kojima, Nakajima, \& Takada, 2008). PIG uses a defined marker set and a set of subject measurements to create joint kinematics and kinetics output. There are a total of 39 markers used in a PIG marker set. The marker locations used in the PIG marker set are shown in Figure 4.8. The 39 markers include 4 markers on the head, 5 markers on the torso, 7 markers on each arm, 4 markers on the pelvis, 4 markers on each leg, and 2 markers on each foot. The anatomical locations of these markers are listed in Table 4.4. In addition to the PIG marker set, four markers (diameter $=3.5 \mathrm{~cm}$ ) were placed on the cart in order to capture its motion relative to the participant during the pushing and pulling tasks (Figure 4.2). 


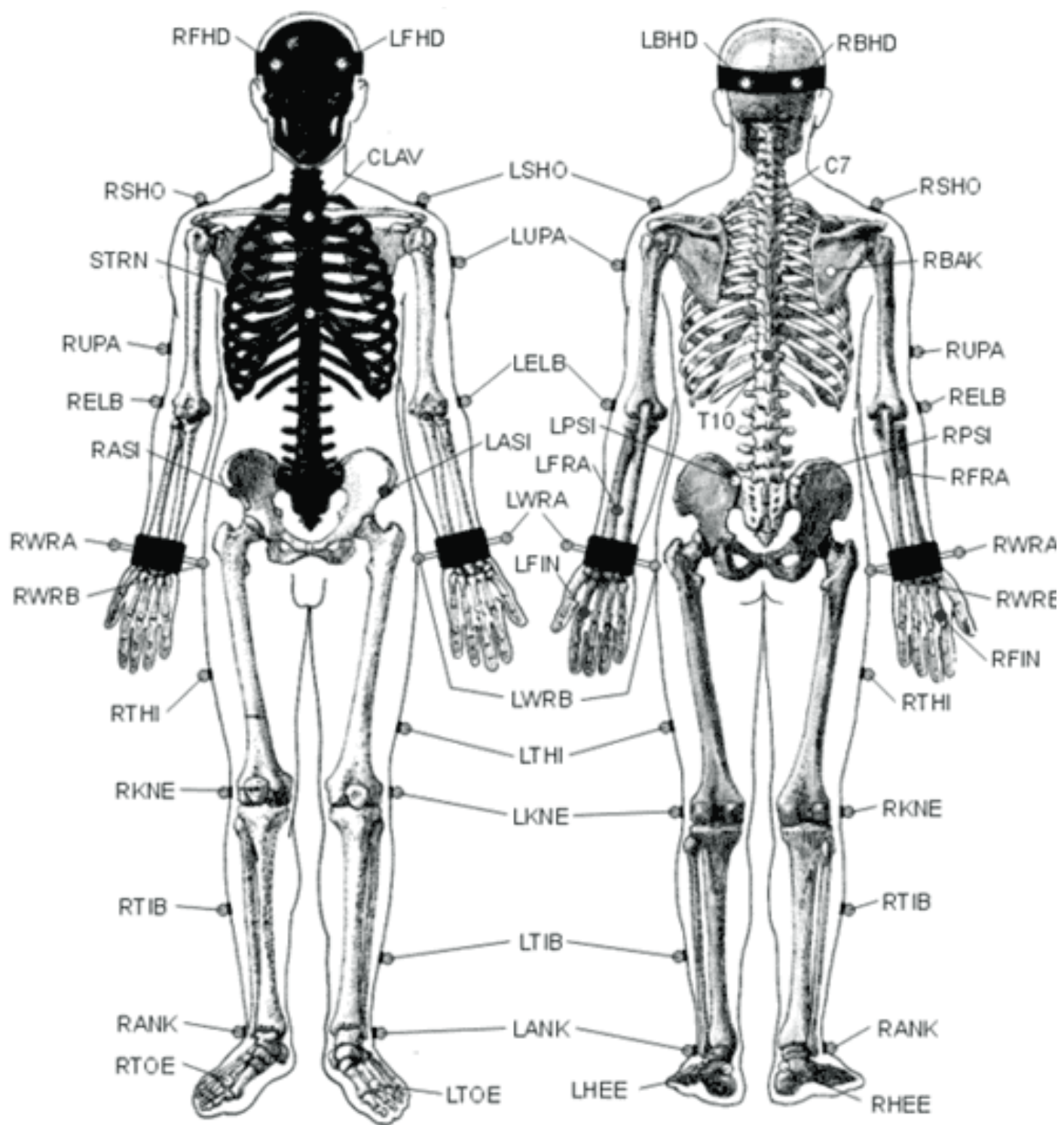

Figure 4.8: Marker locations used in the Plug-in Gait (PIG) marker set 
Table 4.4: Anatomical locations of Plug-in Gait marker set

\begin{tabular}{|c|c|}
\hline \multicolumn{2}{|r|}{ Upper Body Markers } \\
\hline Markers: & Location: \\
\hline LFHD / RFHD & Left and right side of the forehead \\
\hline LBHD / RBHD & Left and right side of the back of the head \\
\hline CLAV & Jugular notch at the proximal aspect of the sternum \\
\hline STRN & Xyphoid process at the distal aspect of the sternum \\
\hline $\mathrm{C} 7$ & Spinous process of the $7^{\text {th }}$ cervical vertebrae \\
\hline RBAK & Mid scapula \\
\hline $\mathrm{T} 10$ & Spinous process of the $10^{\text {th }}$ thoracic vertebrae \\
\hline LSHO / RSHO & Tip of the left and right acromion processes \\
\hline LUPA / RUPA & $\begin{array}{l}\text { Approximately } 1 / 3 \text { of the distance from the shoulder to the elbow, and } \\
\text { slightly to the posterior }\end{array}$ \\
\hline LELB / RELB & Lateral epicondyle of the humerus \\
\hline LFRM / RFRM & Midpoint of the front of the forearm, between the radius and ulna \\
\hline LWRA / RWRA & Medial aspect of the wrist (thumb side) \\
\hline LWRB / RWRB & Lateral aspect of the wrist (pinky side) \\
\hline LFIN/RFIN & Distal aspect of the third metacarpal on the back of the hand \\
\hline \multicolumn{2}{|r|}{ Lower Body Markers } \\
\hline Markers: & Location: \\
\hline LASI / RASI & Anterior superior iliac spine \\
\hline LPSI / RPSI & Lateral aspect of pelvis, at the most superior point of the Ilium \\
\hline LTHI / RTHI & $\begin{array}{l}\text { Approximately } 1 / 3 \text { of the distance from the greater trochanter to the } \\
\text { knee, and slightly to the posterior }\end{array}$ \\
\hline LKNE / RKNE & Lateral condyle of the knee \\
\hline LTIB / RTIB & $\begin{array}{l}\text { Approximately } 1 / 3 \text { of the distance from the knee to the ankle, and } \\
\text { slightly to the posterior }\end{array}$ \\
\hline LANK / RANK & Lateral malleolus of the tibia \\
\hline LHEE/RHEE & On the back of the heel \\
\hline LTOE / RTOE & Distal aspect of the third metatarsal on the top of the foot \\
\hline
\end{tabular}

\subsubsection{AnyBody modeling system}

To quantify the biomechanical loading of the low back and shoulder joints during the pushing/pulling tasks, musculoskeletal models were formulated using the AnyBody modeling system (AnyBody Technology, Aalborg, Denmark). AnyBody modeling system uses AnyScript 
modeling language to formulate detailed musculoskeletal models. The syntax of AnyScript is similar to $\mathrm{C}++$ programming language. It is an object-oriented programming language developed specifically for defining bones (or segments) with different geometries, different types of anatomical joints, muscles with real physiological properties, and an accurate line of actions. The joints in the AnyBody modeling system are driven by using different types of drivers. These drivers can be operated using mathematical functions or experimentally collected kinematic data, and the resulting joint loading is calculated using inverse dynamics analysis.

In biomechanics, especially in gait studies, inverse dynamics analysis is traditionally used to calculate net moments at the lower extremity joints by using experimentally recorded ground reaction forces. Inverse dynamic analysis involves computation of unknown forces by using known motion equations. Due to the redundancy of the muscle actuator configuration, the muscle recruitment problem is formulated as an optimization problem. The usual solution is to recruit muscles to minimize some criterion. The AnyBody modeling system employs a minimum muscle fatigue criterion (Rasmussen, Damsgaard, \& Voigt, 2001), leading to a numerically efficient algorithm for calculating the internal forces in the body. The inverse dynamics analytical process in the AnyBody system is illustrated in Figure 4.8 (Rasmussen, Damsgaard, \& Christensen, 2001). 


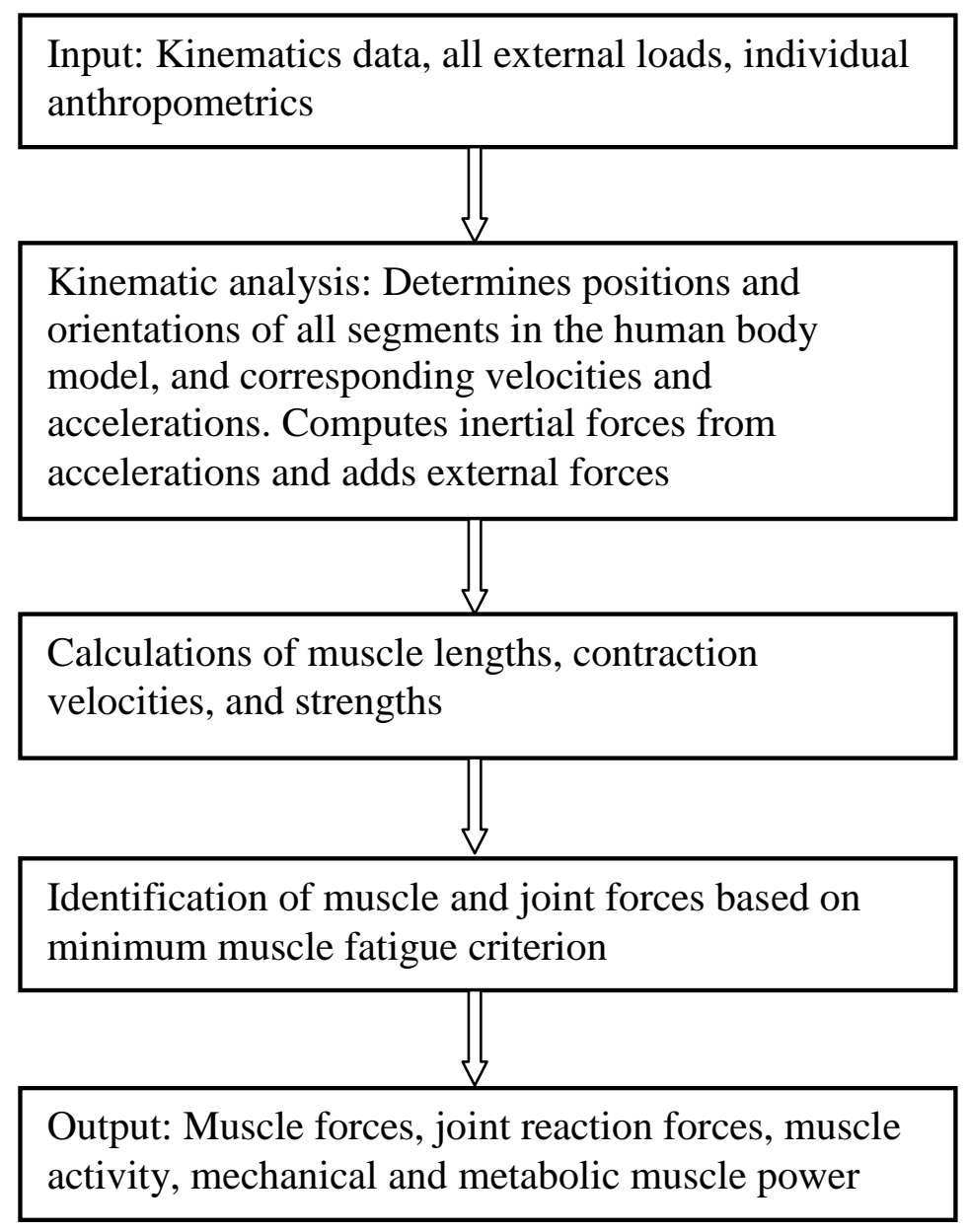

Figure 4.9: Inverse dynamic analytical process implemented by AnyBody modeling system

A public-domain repository of human body models is available which eliminates the need to build the human anatomy over again for each application. By using the model scaling utility, the model can be scaled to match individual anthropometry. The generic AnyBody model comprises of:

(i) an arm and shoulder complex model with 114 muscle fascicles on each side,

(ii) a lumbar spine model comprising the sacrum, all lumbar vertebrae, and a total of 158 muscles fascicles,

(iii) a rigid thoracic spine section,

(iv) a cervical spine model comprising 136 muscles fascicles,

(v) a pelvis and lower extremity model with a total of 326 muscles fascicles. 
Thus, the whole human body model consists of over 750 individual muscle fascicles, 33 joints, and 34 rigid body segments. AnyBody musculoskeletal models have been used previously to estimate musculoskeletal loading during manual material handling tasks (Wagner,

Rasmussen, \& Reed, 2007), shoulder joint forces while operating a wheelchair (Dubowsky, et al., 2008), index finger loading during computer keyboard tapping (Wu, et al., 2008), and lower limb joint movements during stilt walking of construction workers (Wu, Chiou, \& Pan, 2009). The AnyBody modeling system also allows the adding of physical entities in the environment of the human model to evaluate human interaction with different interfaces. Some previous studies have evaluated human interaction with bicycles, furniture, sports equipment, hand tools, etc. In the present study, a hand truck and loads were modeled in the environment file which was connected to the human model.

\subsection{Data Collection Procedures}

The data collection session for each participant consisted of the following steps:

(1) Before the arrival of participants, a set of calibration routines for the optical camera system and force platforms were performed.

(i) Camera calibration: This involved three steps. The first step, static camera calibration, required reading the location of the markers on the static calibration object - "L frame" (Figure 4.10 (a)) by all the cameras. During this step, it was essential that only the "L-frame" markers were visible to the cameras, and no other reflective objects were present in the capture volume. This "L frame" was placed at a specific corner of one of the two force platforms. The second step involved calibrating the capture volume. During this step, the "L-frame" object was removed from the capture volume and a $240 \mathrm{~mm}$ camera calibration wand with three reflective markers (Figure 4.10 (b)) was used. An experimenter slowly moved the wand in a threedimensional motion for about 1 to 2 minutes throughout the entire capture volume to be calibrated. The system then processed the calibration data and reported on the accuracy and precision of the calibrated volume. It is recommended to achieve accuracy (Mean Residual) of less than $1.000 \mathrm{~mm}$ and a static reproducibility of less than $1.000 \%$. The final step of camera calibration involved setting the origin of the capture volume. To set the origin, the "L-frame" 
was placed at the predetermined corner of one of the force plates, and through Vicon Nexus software, proper options were selected to set the origin of the capture volume.

(ii) Force platform calibration: The force plate setting in the Vicon Nexus software was checked for accuracy of the calibration matrix and gain factor. The six analog channels (Fx, Fy, $\mathrm{Fz}, \mathrm{Mx}, \mathrm{My}, \mathrm{Mz}$ ) of each force plates were then zeroed. The experimenter then walked across the plates to ensure that all the plates were feeding correct information to the analog system.
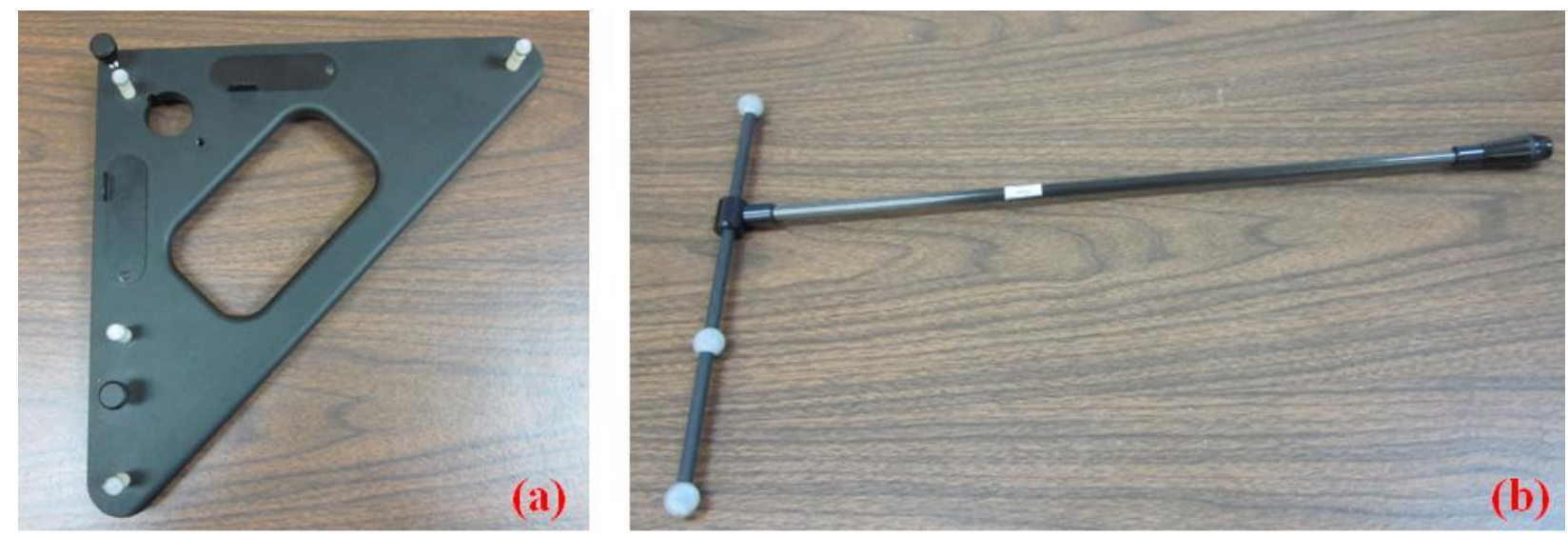

Figure 4.10: (a) Static calibration object - "L frame"; (b) $240 \mathrm{~mm}$ camera calibration wand

(2) Upon participants' arrival to the laboratory, each participant was shown the experimental set up (walkway, cart, force plates, optical motion capture system, and loads to be transferred with cart) and a brief explanation of the research objectives was provided. They were then asked to read and sign an informed consent form, in accordance with the West Virginia University’s IRB policy statement.

(3) Participants then changed to the test shirt and shorts and a set of demographic and anthropometric measurements were recorded. A list of anthropometric variables measured in this study is shown in the Table 4.5. Some of these measurements are required by Vicon Nexus software to form a real time stick figure of the participants during data collection, while others are required to formulate the model using the AnyBody system. 
Table 4.5: Required subject anthropometric measurements

\begin{tabular}{|c|c|c|}
\hline & \multicolumn{2}{|c|}{ Required subject measurements } \\
\hline & For Vicon & For AnyBody modeling \\
\hline \multirow{2}{*}{ General } & \multicolumn{2}{|c|}{ Body mass (in kg) } \\
\hline & \multicolumn{2}{|c|}{ Height (in cm) } \\
\hline $\begin{array}{c}\text { Upper } \\
\text { body } \\
\text { (in cm) }\end{array}$ & $\begin{array}{l}\text { Shoulder offset: vertical distance from the } \\
\text { center of the glenohumeral joint to the } \\
\text { marker on the acromion clavicular joint } \\
\text { Elbow width: distance between the medial } \\
\text { and lateral epicondyles of the humerus } \\
\text { Wrist width: distance between the ulnar } \\
\text { and radial styloids } \\
\text { Hand thickness: distance between the } \\
\text { dorsal and palmar surfaces of the hand }\end{array}$ & $\begin{array}{l}\text { Head height: height in neutral position from } \\
\text { C1Hat node to top of head } \\
\text { Trunk height: C1Hat node to L5S1 joint } \\
\text { Upper arm length: distance in neutral } \\
\text { position from acromioclavicular joint to } \\
\text { elbow joint } \\
\text { Forearm length: distance in neutral position } \\
\text { from elbow joint to wrist joint } \\
\text { Hand length: distance from wrist to tip of } \\
\text { fingers } \\
\text { Hand breadth: distance between the medial } \\
\text { and lateral sides of the hand }\end{array}$ \\
\hline $\begin{array}{c}\text { Lower } \\
\text { body } \\
\text { (in cm) }\end{array}$ & $\begin{array}{l}\text { Leg length: distance between ASIS and } \\
\text { medial malleolus, via the knee joint } \\
\text { Knee width: distance between the lateral } \\
\text { and medial femoral epicondyles } \\
\text { Ankle width: distance between the medial } \\
\text { and lateral malleoli }\end{array}$ & $\begin{array}{l}\text { Pelvis width: distance between hip joints } \\
\text { Thigh length: distance between ASIS to } \\
\text { knee joint } \\
\text { Shank length: distance between knee joint } \\
\text { and ankle joint } \\
\text { Foot length: distance between the heel and } \\
\text { the toe of the foot }\end{array}$ \\
\hline
\end{tabular}

(4) The next step in the data collection was to attach the thirty-nine reflective markers on the participant at various anatomical locations using the Vicon Plug-in Gait marker set. The Vicon Skeleton Template (.vst), which is the generic definition of the human skeleton, was used for performing the static subject calibration. The original Vicon Skeleton Template "Plug-in Gait full body.vst" was modified by adding a cart segment with four markers to create a "Cart.vst" model template. This modified template file was then used for collecting and processing the kinematic data.

(5) Prior to the initiation of experimental testing, the participant stood in a static T-pose to be captured by Vicon as a calibration trial. No motion capture should be started until verification that all tracking markers were visible during this step. Upon finishing the calibration trial, participants performed several practice trials to become familiar with the experimental tasks. 
(6) The participants then performed the experimental trials. The trial order was randomized. Participants were instructed to begin each push/pull task with both feet together, by standing straight and holding the cart handle with two hands. At a verbal signal from the experimenter, participants pushed/pulled the cart along the walkway from the start to the ending position. The data were recorded for each trial during the experiment and stored on the computer for later data processing. Duration of a trial was approximately 15 to 20 seconds. A rest period of 1 minute was provided between the trials. Owing to the submaximal nature of the exertions, this rest period was sufficient to allow full recovery. A participant mounted with the reflective markers performing cart pushing/pulling experimental trials is shown in Figure 4.11.
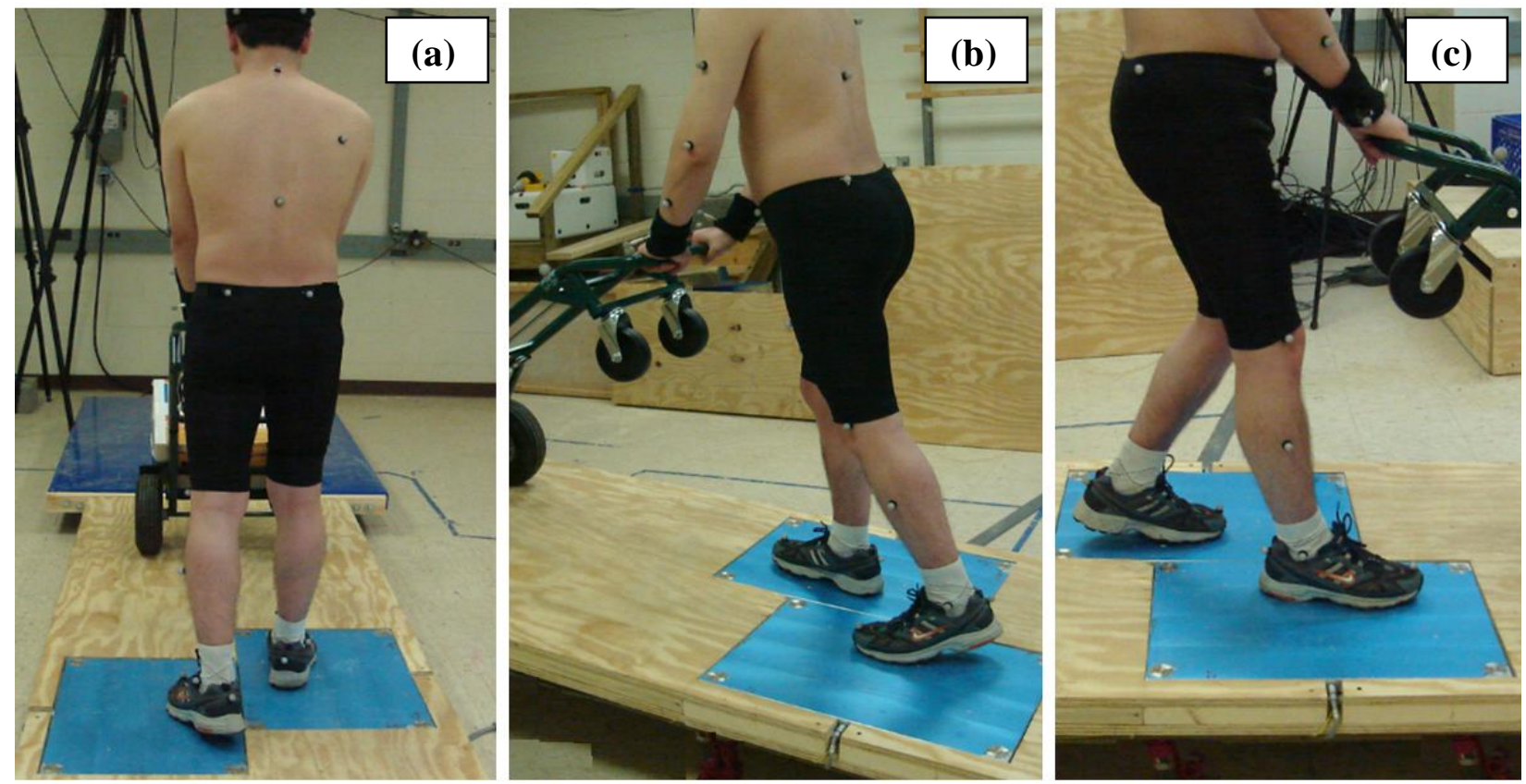

Figure 4.11: Participant performing cart pushing/pulling tasks along (a) a flat walkway, (b) a ramped walkway while going uphill, and (c) a ramped walkway while going downhill

\subsection{Data Processing}

\subsubsection{Kinematic data: Vicon Nexus software}

The marker data were processed using Vicon Nexus motion software. In this software, each marker was labeled and a corresponding human model was created by performing the steps as follows: 


\section{(i) Reconstruction and cropping:}

The 3D positions were computed by reconstruction of the trial. It may take time to fully reconstruct the entire trial. Before labeling the trajectories in the motion trials, unwanted data that were outside the duration of the analysis were cropped.

\section{(ii) Trajectory identification and gap filling:}

To label the trajectories, a static $3 \mathrm{D}$ view of the marker is used. The markers were identified manually by selecting a marker from the 3D view and then selecting its four letter marker name from the list obtained from the .vst model template. As the markers were identified, blue lines were added automatically to join markers that are part of the head and trunk segment, whereas red and green lines added to joint markers of the left and right upper/lower body, respectively. The four markers of the cart were also identified and added with yellow lines. Once all markers were identified, we checked that all the trajectories were joined without any gaps. If a marker turned white, it was manually labeled. Next, all the gaps were filled and any unlabeled trajectories were deleted. Figure 4.12 shows the last step of checking the marker labeling and gap filling. Finally, the trial data were exported and saved in C3D file format, which consisted of 43 markers and force plates' analog data. The C3D file was imported to AnyBody modeling system to run the full body musculoskeletal model.

\section{(iii) Angle outputs:}

The Vicon Nexus software can output the joint angles of major body joints, such as the trunk, hip, knee, ankle, shoulder, elbow, and wrist. The output angles for all joints were calculated from YXZ cardan angles derived by comparing the relative orientations of the two segments (Vicon). In the current study, the peak flexion/extension values of trunk, right shoulder, right elbow, right hip, right knee, and right ankle were exported. Table 4.6 shows the angle definitions for the exported flexion/extension angles. 

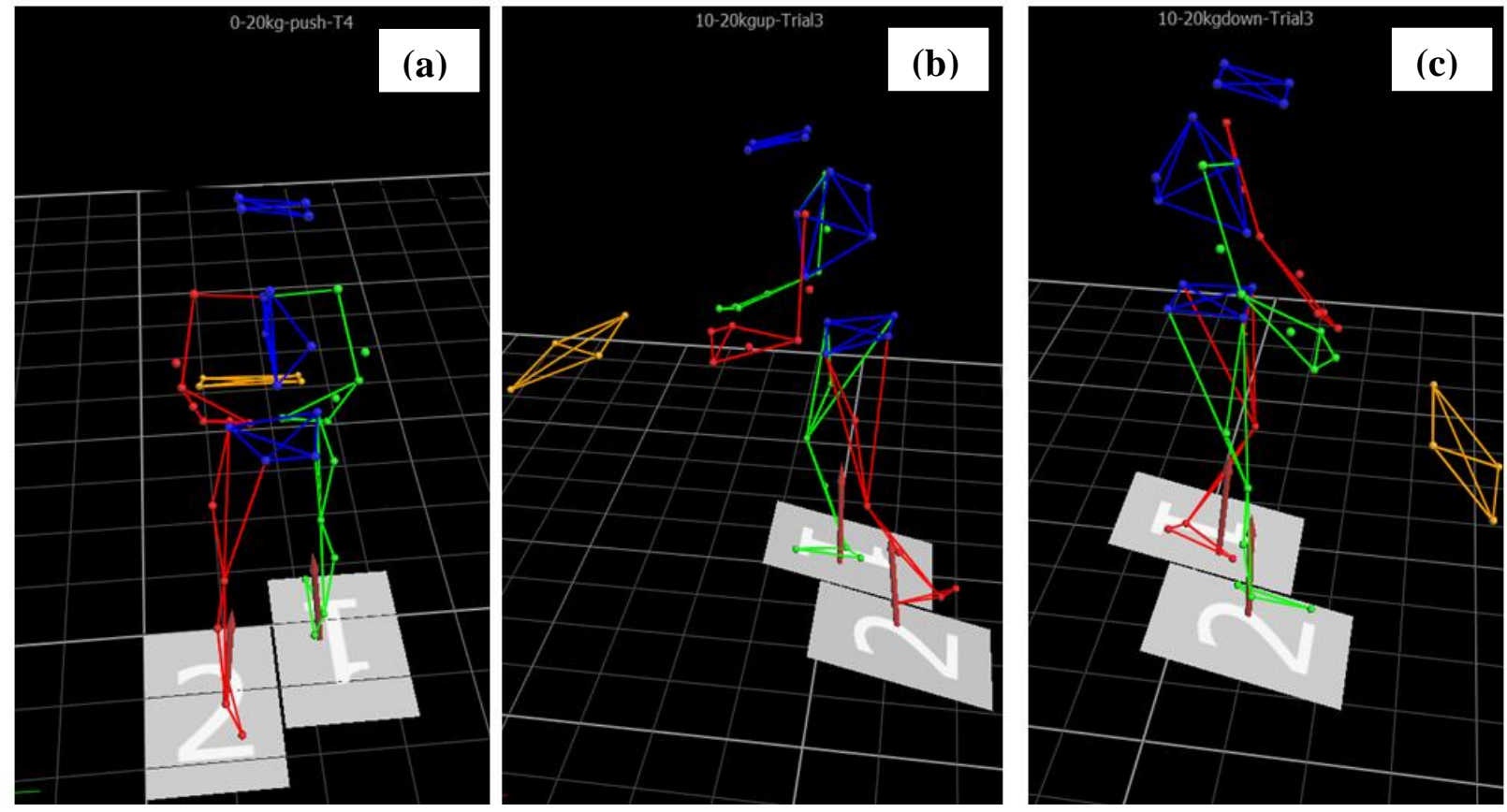

Figure 4.12: The human model performing cart pushing/pulling tasks created by Vicon Nexus software:(a) pushing along the flat walkway; (b) uphill pushing along a ramp; (c) downhill pulling along a ramp

Table 4.6: Definitions of exported joint angles

\begin{tabular}{|l|l|}
\hline \multicolumn{1}{|c|}{ Angle } & \multicolumn{1}{c|}{ Definition } \\
\hline Frunk & $\begin{array}{l}\text { Trunk flexion is the angle between the sagittal thorax axis and the sagittal pelvis } \\
\text { axis around the fixed transverse axis of the pelvis. A positive (Flexion) angle value } \\
\text { corresponds to the situation in which the thorax is tilted forward. }\end{array}$ \\
\hline $\begin{array}{l}\text { Shoulder } \\
\text { Flexion/Extension }\end{array}$ & $\begin{array}{l}\text { Shoulder flexion is calculated about an axis parallel to the thorax transverse axis. } \\
\text { Shoulder flexion is the angle between the projected sagittal-humerus axis and the } \\
\text { sagittal-thorax axis around the fixed transverse axis of the thorax. A positive } \\
\text { (Flexion) angle value corresponds to the situation in which the arm is in front of the } \\
\text { body. }\end{array}$ \\
\hline $\begin{array}{l}\text { Elbow } \\
\text { Flexion/Extension }\end{array}$ & $\begin{array}{l}\text { Elbow flexion is the only kinematic parameter calculated at the elbow as the } \\
\text { segment definitions of the Humerus and radius result in two of the axes being } \\
\text { shared. Elbow flexion is calculated between the sagittal radius axis and the sagittal } \\
\text { humerus axis around the fixed transverse axis of the humerus. A positive number } \\
\text { indicates a flexion angle. }\end{array}$ \\
\hline $\begin{array}{l}\text { Hip } \\
\text { Flexion/Extension }\end{array}$ & $\begin{array}{l}\text { Hip flexion is calculated about an axis parallel to the pelvic transverse axis which } \\
\text { passes through the hip joint centre. The sagittal thigh axis is projected onto the } \\
\text { plane perpendicular to the hip flexion axis. Hip flexion is then the angle between } \\
\text { the projected sagittal thigh axis and the sagittal pelvic axis. A positive (Flexion) } \\
\text { angle value corresponds to the situation in which the knee is in front of the body. }\end{array}$ \\
\hline $\begin{array}{l}\text { Knee } \\
\text { Flexion/Extension }\end{array}$ & $\begin{array}{l}\text { The sagittal shank axis is projected into the plane perpendicular to the knee flexion } \\
\text { axis. Knee Flexion is the angle in that plane between this projection and the sagittal } \\
\text { thigh axis. The sign is such that a positive angle corresponds to a flexed knee. }\end{array}$ \\
\hline $\begin{array}{l}\text { Ankle } \\
\text { Flexion/Extension }\end{array}$ & $\begin{array}{l}\text { The foot vector is projected into the foot sagittal plane. The angle between the foot } \\
\text { vector and the sagittal axis of the shank is the Foot Dorsi/Plantar Flexion. A } \\
\text { positive number corresponds to dorsiflexion. }\end{array}$ \\
\hline
\end{tabular}




\subsubsection{Musculoskeletal model simulation}

The AnyBody modeling system caters to a wide range of users with very different modeling needs. Some may simply want to load and run an existing model, some may need to modify the model to a smaller or larger extent, and some may want to build their own model bottom-up. Most users can find themselves in one of the following scenarios:

- Loading a model from the model repository and changing simple parameters like the load.

- Modifying one of the existing motion capture models from the repository to the lab setup.

- Modifying a model from the repository by redefining the environment.

- Constructing a body model and its environment bottom-up.

In the present study, the musculoskeletal modeling was based on one of the existing motion capture models (Gait Full Body Model) from the model repository (Version 1.2 of AnyBody Managed Model Repository, AMMRV1.2). The structure of the repository is shown in Figure 4.13:

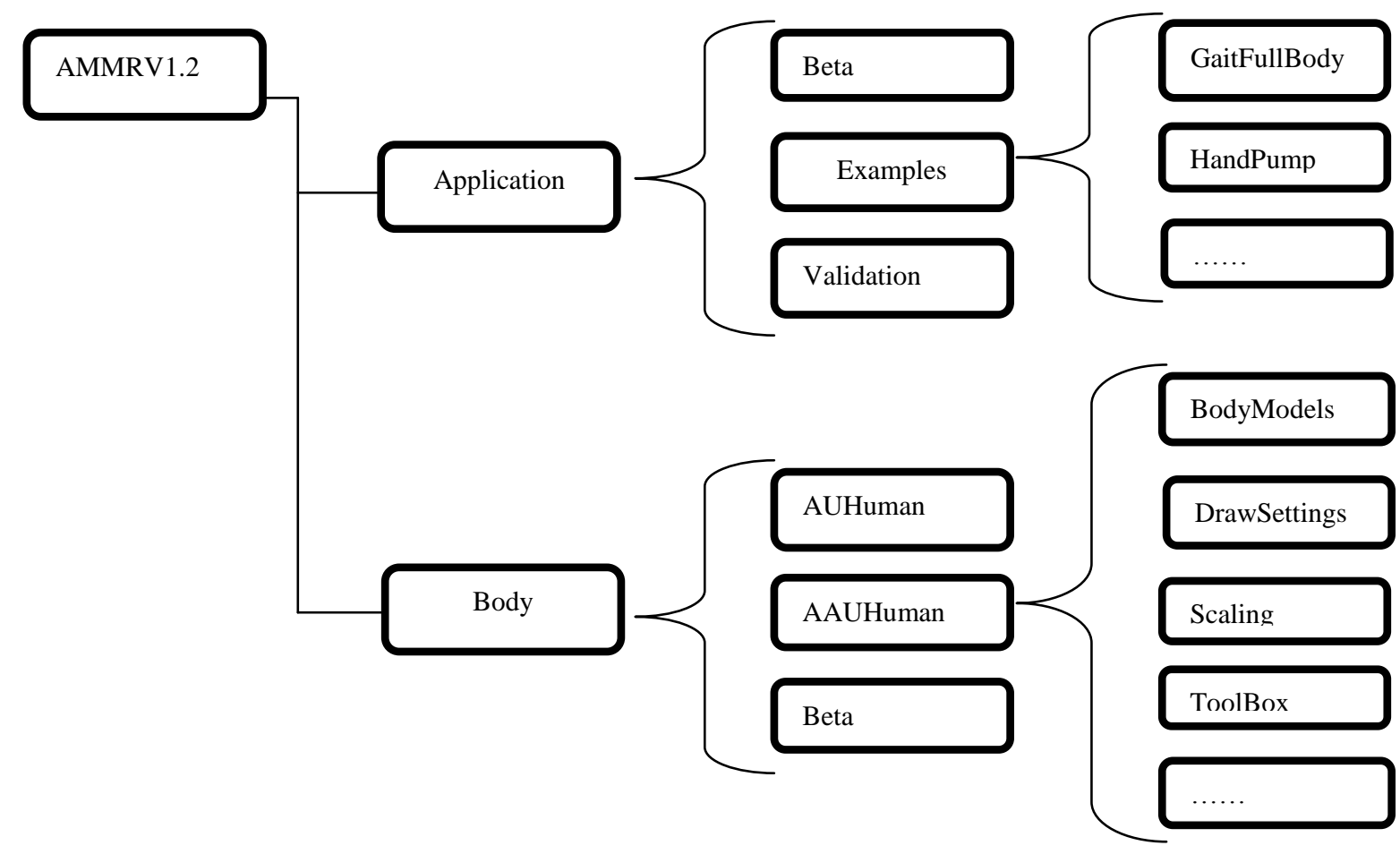

Figure 4.13: Structure of AnyBody repository 
Note: (1) Applications are the models that can be loaded into AnyBody and simulate biomechanical situation.

(2) Body contains models of body parts and collection of body parts which are used by the applications. The entire body model contains many hundreds of muscles, and it is rather heavy computationally.

(3) Beta folder contains models that are unfinished but may be of value to users with special interests.

(4) Example folder contains many models of different situations.

(5) Validation folder contains models that have been used for validation purposes, typically by comparison of the model predictions with experimental measurements.

(6) AAUHuman contains the Aalborg university full body model, while AUHuman contains a mandible model developed in Arhus University, and Beta refers to other research groups and the non-human models.

We modified the Gait Full Body model to our own laboratory setup by redefinition of the marker protocol (adding four markers of the cart), redefinition of the environment (the force platforms, the cart as an additional segment definition), and matching initial posture and individual anthropometrics for each participant. The modified "Cart Push Pull" model was loaded to simulate the dynamic cart pushing/pulling tasks. A kinematic optimization and inverse dynamic analysis was then operated; lastly, the joint loading in the form of joint reaction forces of low back at L5S1 level and three major shoulder joints (SC, AC, and GH) was saved and exported into a data sheet. Figure 4.14 shows the structure of the model with the kinematic and inverse dynamic studies used for running and quantifying the musculoskeletal loading, respectively. 


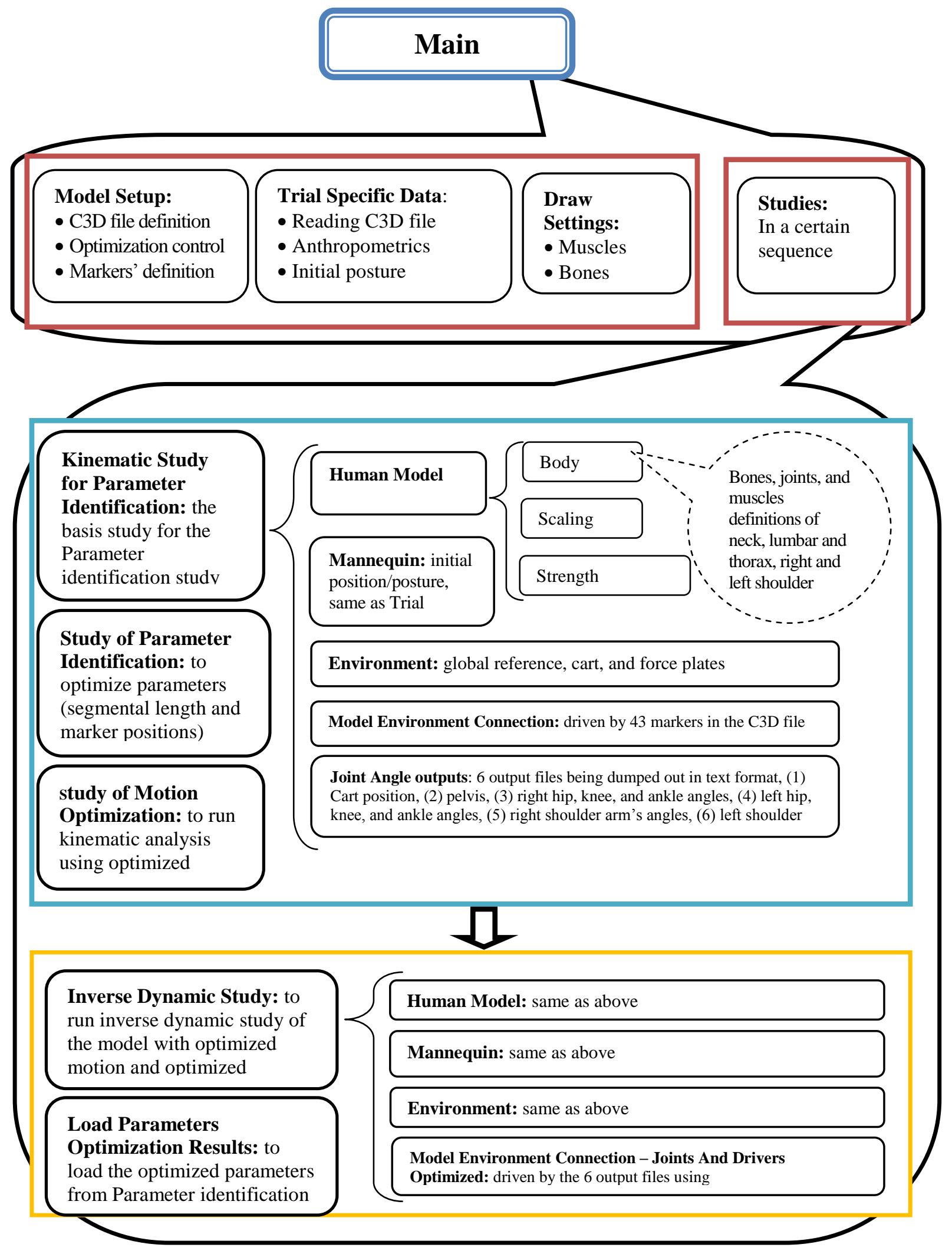

Figure 4.14: Structure of the musculoskeletal model and details of the kinematic and inverse dynamic analysis studies 
The following sections provide a detailed description of the model.

\subsubsection{1 “Model Setup"}

"Model Setup" file contains coding for the definition of the input file (C3D file), the marker topology, and linkage between the free floating markers in the C3D file with the markers on the human body.

(i) Definition of the input file to the analysis:

This step was executed by using "AnyInputC3D”, "AnyString" and "AnyFunButterworthFilter" terms to connect to the C3D file.

(ii) Redefinition of the marker topology of the C3D file:

The existing file contains the definition of thirty-nine markers shown in Figure 4.8: Plugin Gait (PIG) marker placement protocol. However, the present study had four more markers attached on the cart to capture the motion of the cart during the tasks, shown in Figure 4.2. Therefore, we will add the definitions of the four markers of the cart in the "Model Setup" file. The collected C3D file from the optical motion analysis system was named as "CARTRU," “CARTLU," “CARTRL,” and "CARTLL," which are defined as below:

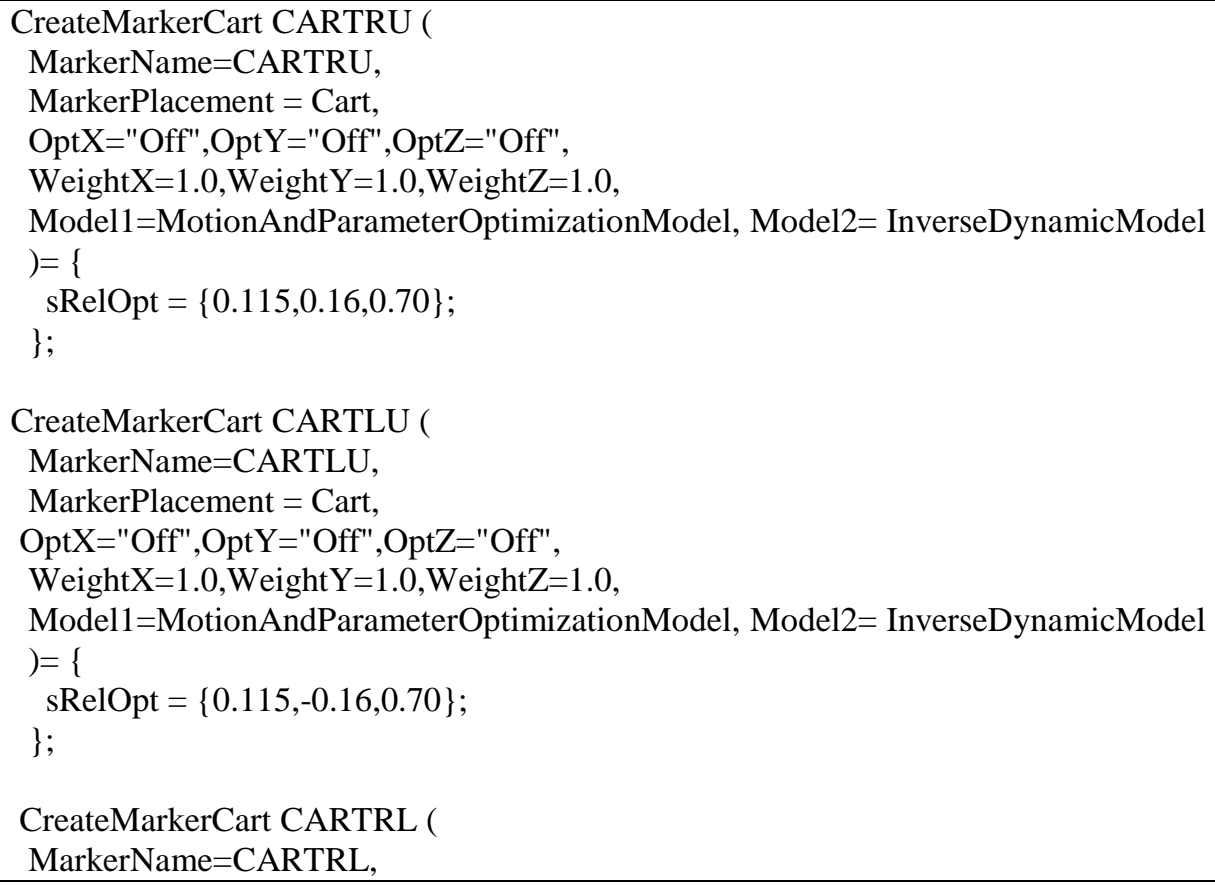




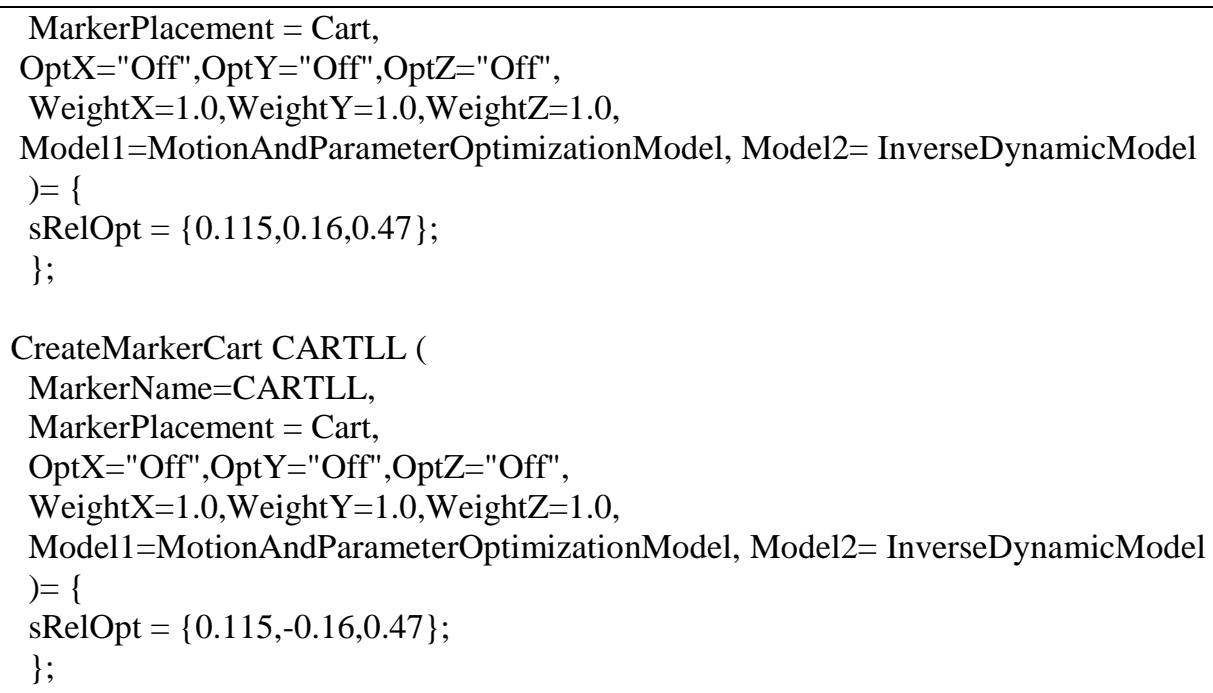

Figure 4.15: Model statement for defining the four markers attached on the cart

sRelOpt is the relative position of the marker at the cart interface. The three coordinate values ( $\mathrm{x}, \mathrm{y}$, and $\mathrm{z}$ ) were input according to the measurement between the center of the mass (cart and load) and marker positions.

(iii) Optimization control about anthropometrics and marker location:

Except the head height and varus-valgus anthropometrics, other segmental anthropometrics data such as pelvis width, thigh length, shank length, foot length, trunk height, upper arm length, and lower arm length were optimized when running the kinematic optimization study. The AnyScript like "OptX," "OptY," and "OptZ," shown in the definition of the above "CARTRU" marker box, determines if the design variable (x, y, or z coordinate of sRelOpt) was to be optimized (“On”) or left unchanged in parameter optimization study (“Off”).

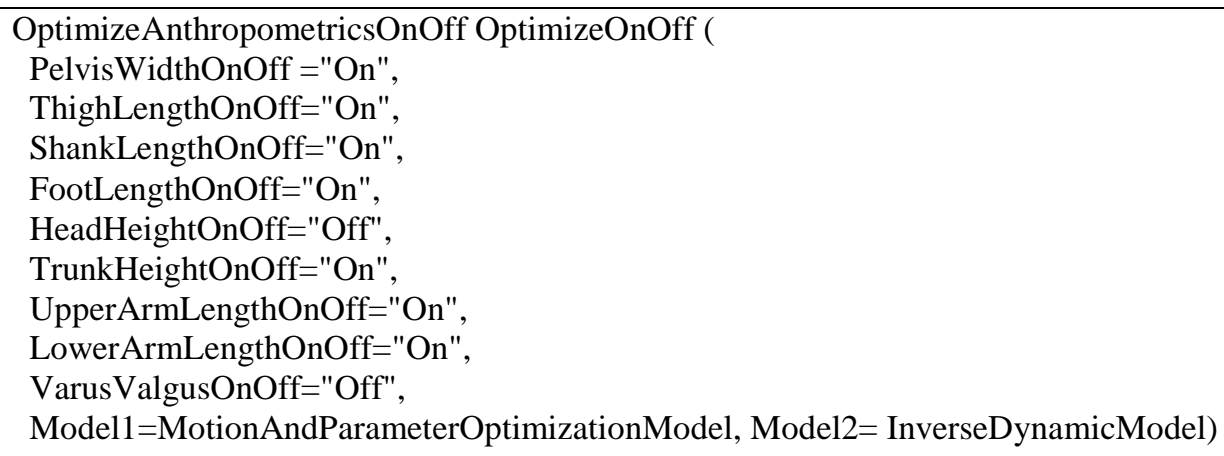

Figure 4.16: Model statement for parameter optimization control 


\subsubsection{2 "Trial Specific Data"}

(i) Reading the C3D data file for the analysis:

This step was executed by using the "AnyString" term to input the C3D file name (e.g., "Subject name - 0 degree - $20 \mathrm{~kg}$ - Trial 1", "Subject name - 5 degree - $20 \mathrm{~kg}$ - pushup - Trial 1", "Subject name - 10 degree - $20 \mathrm{~kg}$ - pushup - Trial 1", etc.)

(ii) Controlling the start and end time:

It was essential to identify the frames in the C3D file corresponding to the heel contact of the first foot with the force platform and toe off of the other foot from the force platform, to accurately run the model. These frames were identified manually and assigned to the tstart and tend values in the trial specific part of the model.

AnyString NameOfFile ="YY-5 degree -20kg-pushup-Trial1"; // to change the name of the C3D file accordingly

//Starting time for the analysis calculated from C3D data but can be changed is needed

AnyFloatVar tStart =

Main.ModelSetup.C3DFileData.Header.FirstFrameNo/Main.ModelSetup.C3DFileData.Header.VideoFrameRate+1.

7; //to start from the time the first foot touching the plate by adjusting the time manually

//End time for the analysis calculated from C3D data but can be changed is needed

AnyFloatVar tEnd =

Main.ModelSetup.C3DFileData.Header.LastFrameNo/Main.ModelSetup.C3DFileData.Header.VideoFrameRate-

$0.15 ; / /$ to end from the time the last foot leaving the plate by adjusting the time manually

Figure 4.17: Model statements for controlling file name, start and ending time

(iii) Input individual anthropometrics data:

Here the anthropometric measurements were input to match each participant. These data were used as an initial guess for the optimization algorithm that would allow changes to the segmental lengths in the study of kinematic optimization.

\footnotetext{
AnyFolder Anthropometrics $=\{$

AnyVar BodyMass=63; //the mass is automatically distributed to the segments

AnyVar BodyHeight=1.72;//height

AnyVar ThighLength $=0.47 ; / /$ right and left side is mirrored

AnyVar ShankLength $=0.38$;

AnyVar FootLength=0.27;

AnyVar PelvisWidth=0.23; //distance between hip joints

AnyVar HeadHeight $=0.15$; / / height in neutral position from $\mathrm{C} 1$ HatNode to top of head

AnyVar TrunkHeight $=0.54$;//height in neutral position from $\mathrm{C} 1$ HatNode to L5SacrumJnt
} 
AnyVar UpperArmLength $=0.275$;

AnyVar LowerArmLength $=0.27$;

AnyVar HandLength $=0.182$;

AnyVar HandBreadth $=0.088$;

\}

Figure 4.18: Model statement for inputting individual anthropometric measurements

(iv) Input initial position of body and its posture

The position of the human body, which is defined by angles of "PelvisRotZ," "PelvisRotY," and "PelvisRotX," was adjusted to the C3D data's XYZ coordinate system. Then based on the joint configuration in the frame corresponding to the tstart, the joint angles were adjusted to minimize the error in the marker locations at the load time.

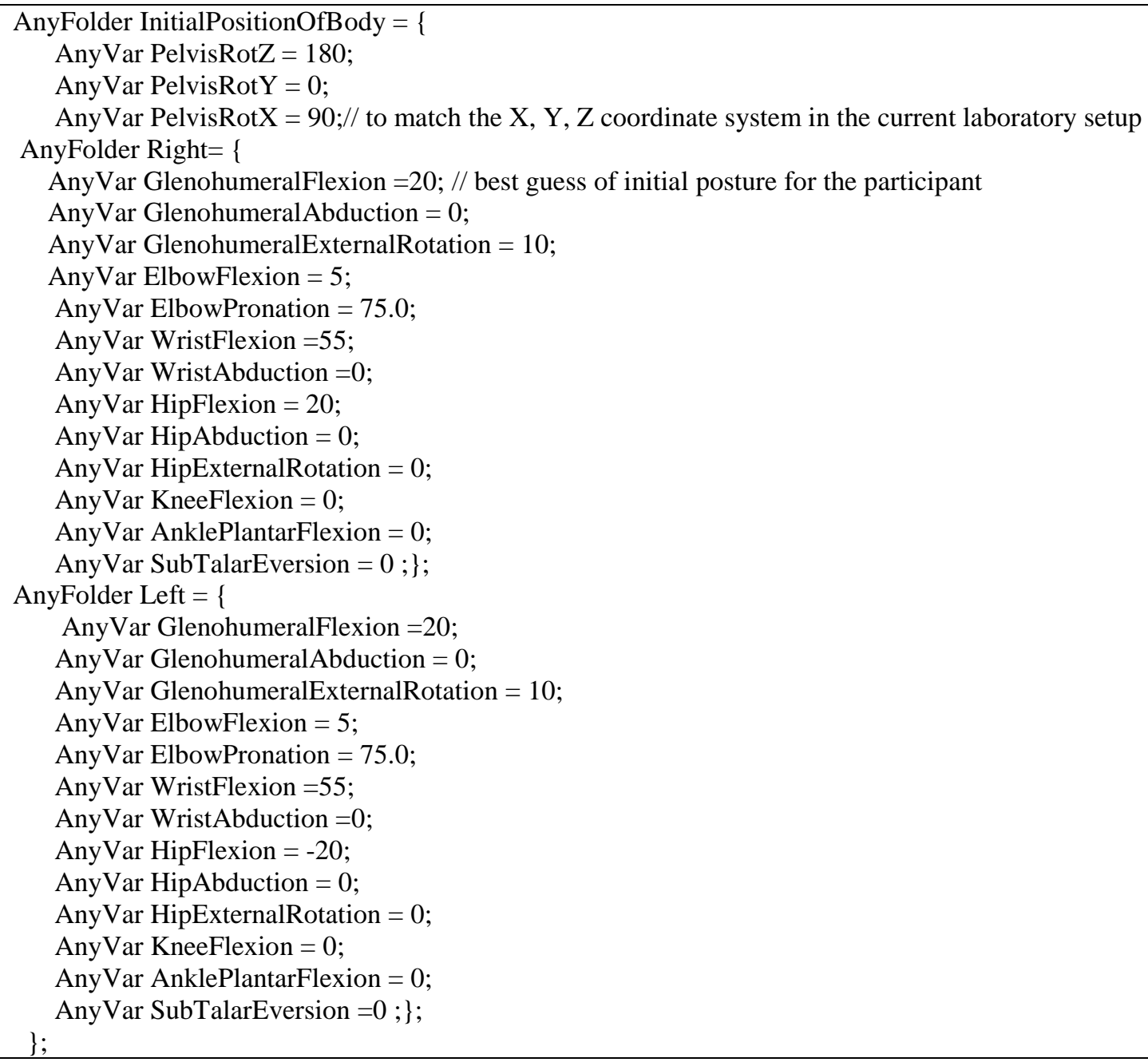

Figure 4.19: Model statement for initial position and posture for participant 


\subsubsection{3 "Environment" around the human}

The environment around the human was modified by adding a hand cart, reaction forces at the hand cart interface, two force plates and the corresponding ground reaction forces.

(i) Creating the hand cart as an additional segment

The cart was defined as a segment in the environment file by using the "AnySeg" object in AnyScript. It was defined as a rigid body, formed using eighteen nodes joined together using polyline object. Additionally, two nodes for the right and left handle were created to connect the cart with the hands. The mass properties of the cart were defined by means of a mass and an inertia tensor.

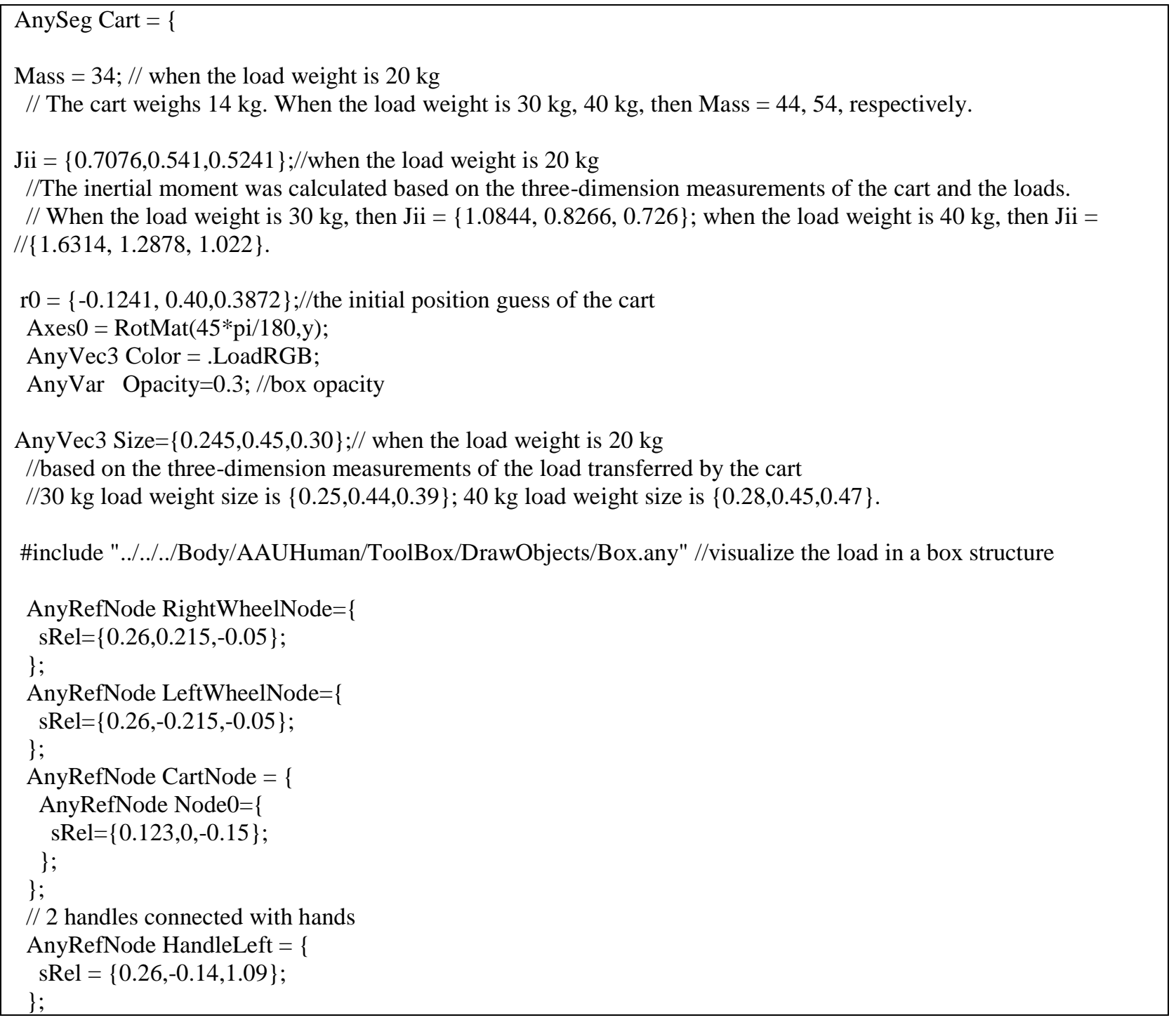




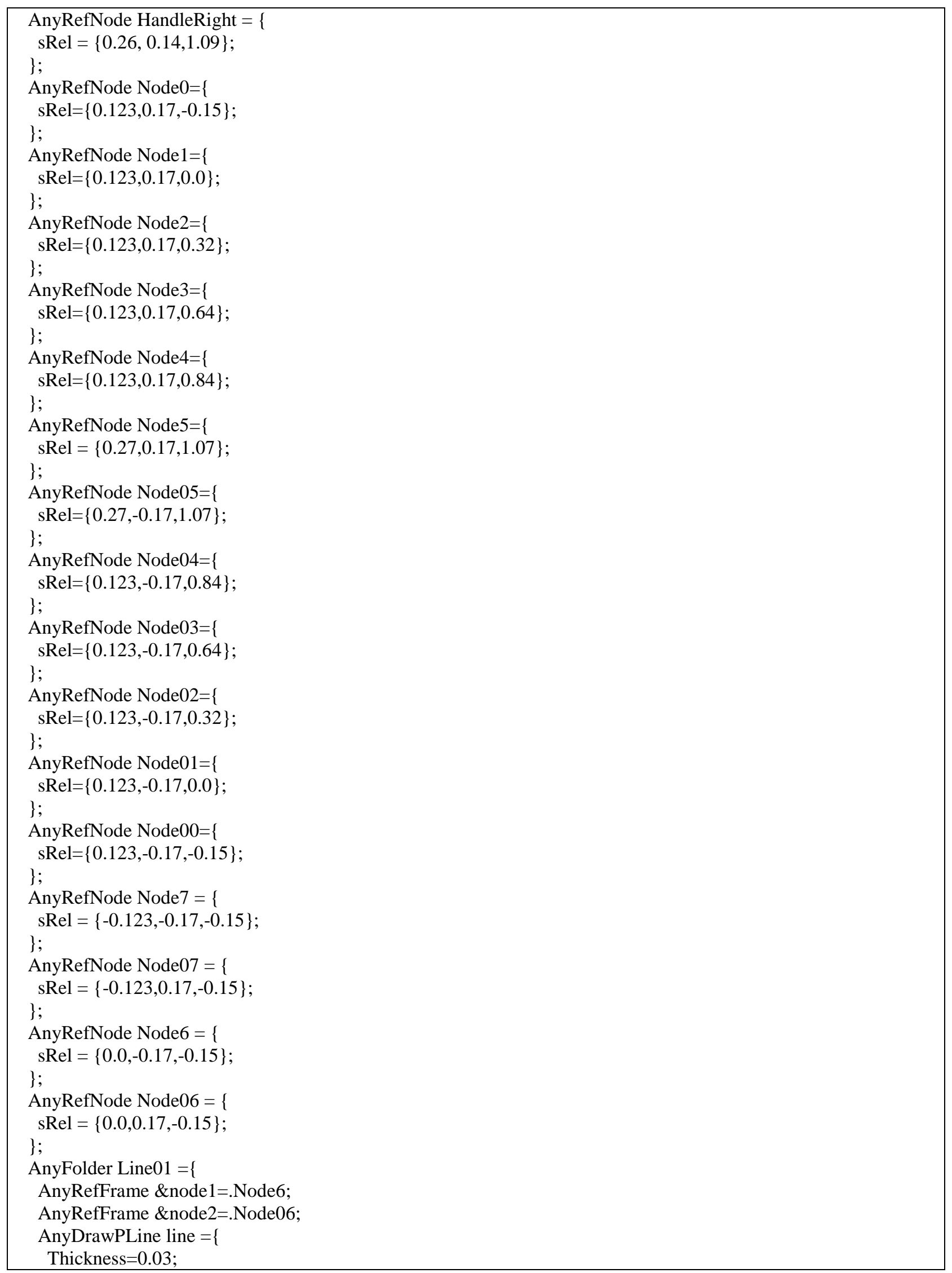




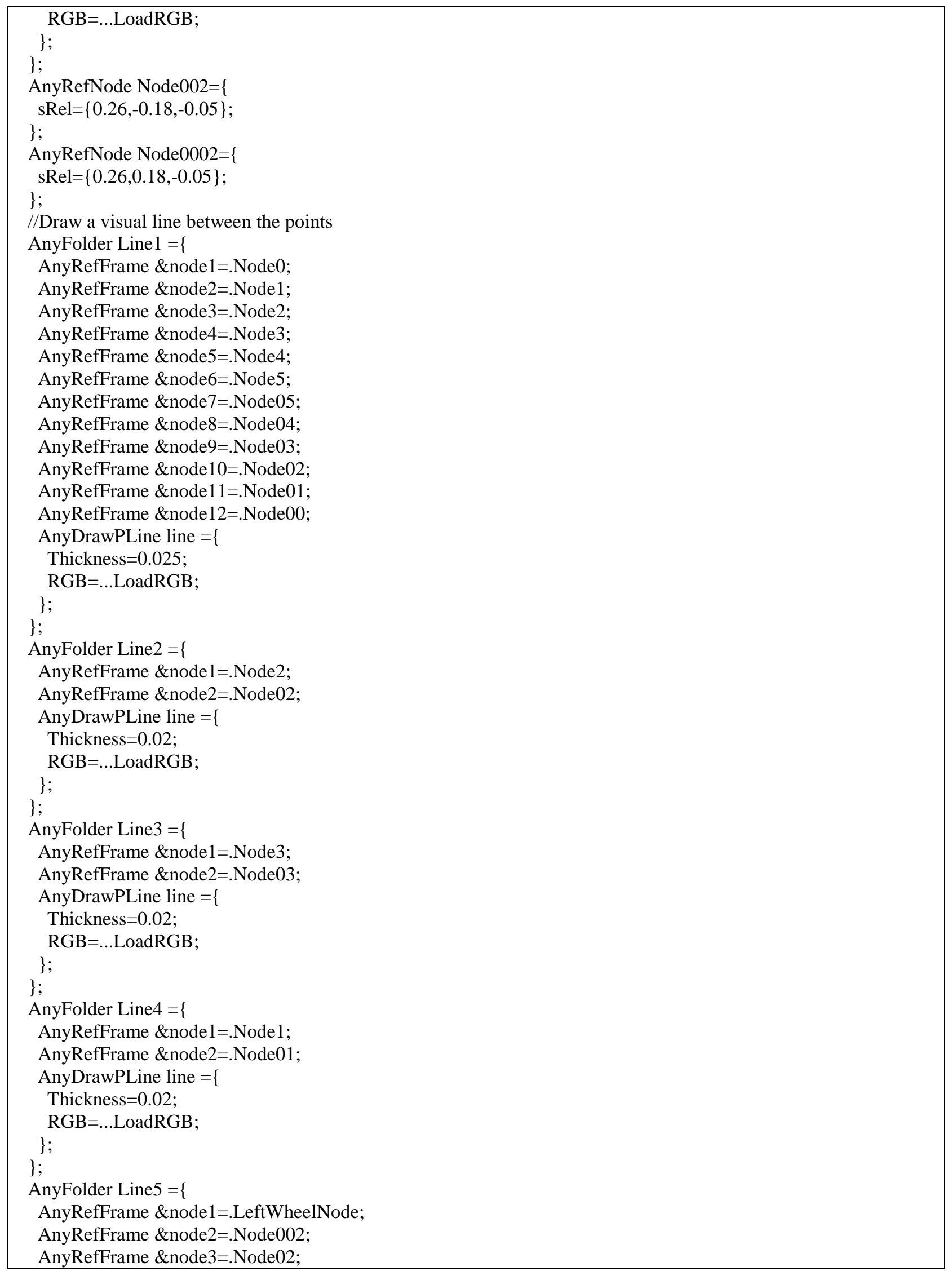




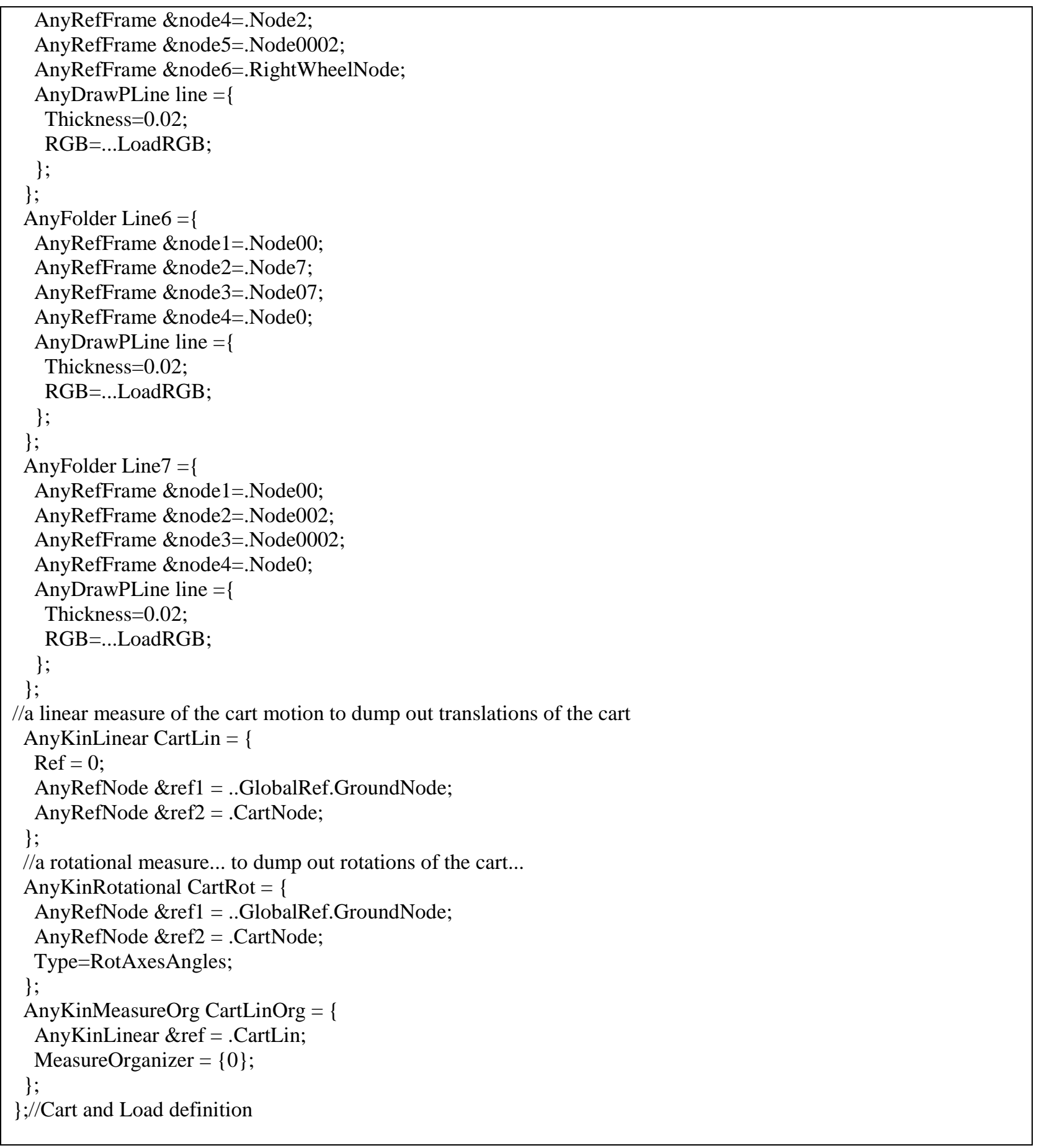

Figure 4.20: Model statement for definition and visualization of the hand cart used in the study 


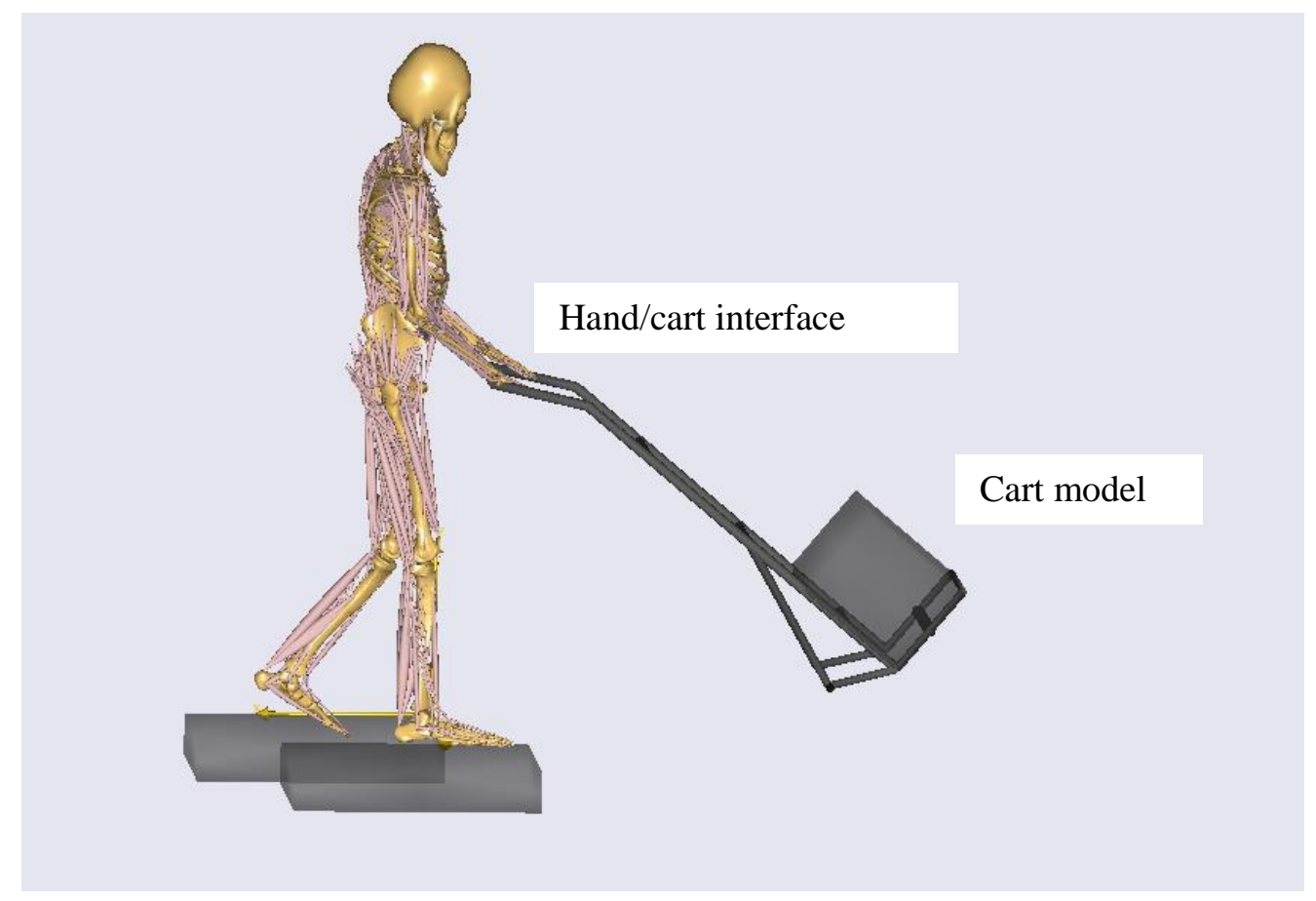

Figure 4.21: Cart model and hand/cart interface visualized in AnyBody modeling system

(ii) Definition of hand/cart interface with kinematic measures and contact forces

This step created kinematic measures ("AnyKinLinear" and "AnyKinRotational") that allow movement tracking of the palm joint of each hand with respect to the cart. An AnyKinLinear measure can gauge the spatial vector between two points (nodes). The line Ref = 0 means that the coordinates of this linear distance were measured first in the coordinate system of the measure's end points, which in this case was the cart's right handle frame. An AnyKinRotational measure can gauge the rotation vector between two points. The contact force between the hand and the cart handle was added by using the "AnyReacForce" object.

The force equilibrium in the AnyBody modeling system is taken care of by the muscles and the reaction forces in the model. The reactions come as joint reactions or as AnyReacForce used in this study. The muscle recruitment algorithm attempts to lower the highest activated muscle; in this process the reaction forces are determined automatically. The reaction forces had no cost in the objective function, so the algorithm always tries to use the available reactions in order to lower the muscle activations as much as possible. Therefore, the AnyScript programming here for the hand/cart interface formulation was in principle a two-step procedure, 
first the kinematic measures between the hand and the cart were solved, then the kinetics were resolved by determining the reaction forces at the hand/cart interface.

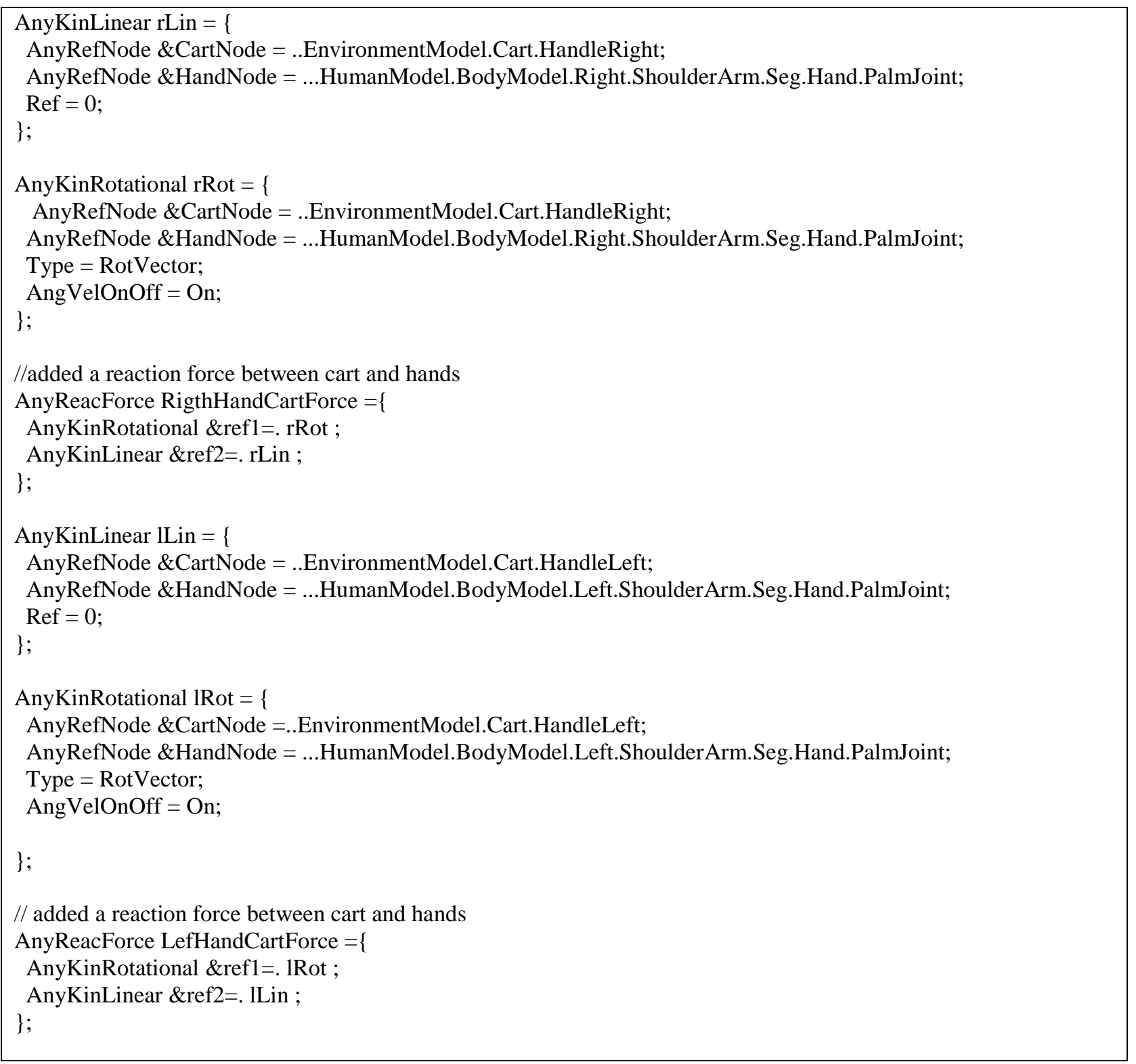

Figure 4.22: Model statement for the hand/cart interface formulation

(iii) Creating two force plates, and automatic detection of foot with correct force plate

The default force plate type in the Gait Full Body model was set as "Type 4," which was the same as a "Type 2" force platform, except that a calibration matrix was being provided for it via the CAL_MATRIX parameter. Our C3D file does not have a calibration matrix data; as a result, the model was modified to execute a "ForcePlateType2AutoDetection" term to simulate 
the two force plates and connected to the C3D file data (Fx, Fy, Fz, Mx, My, Mz). The term could automatically detect foot contact with force plate 1 or plate 2 .

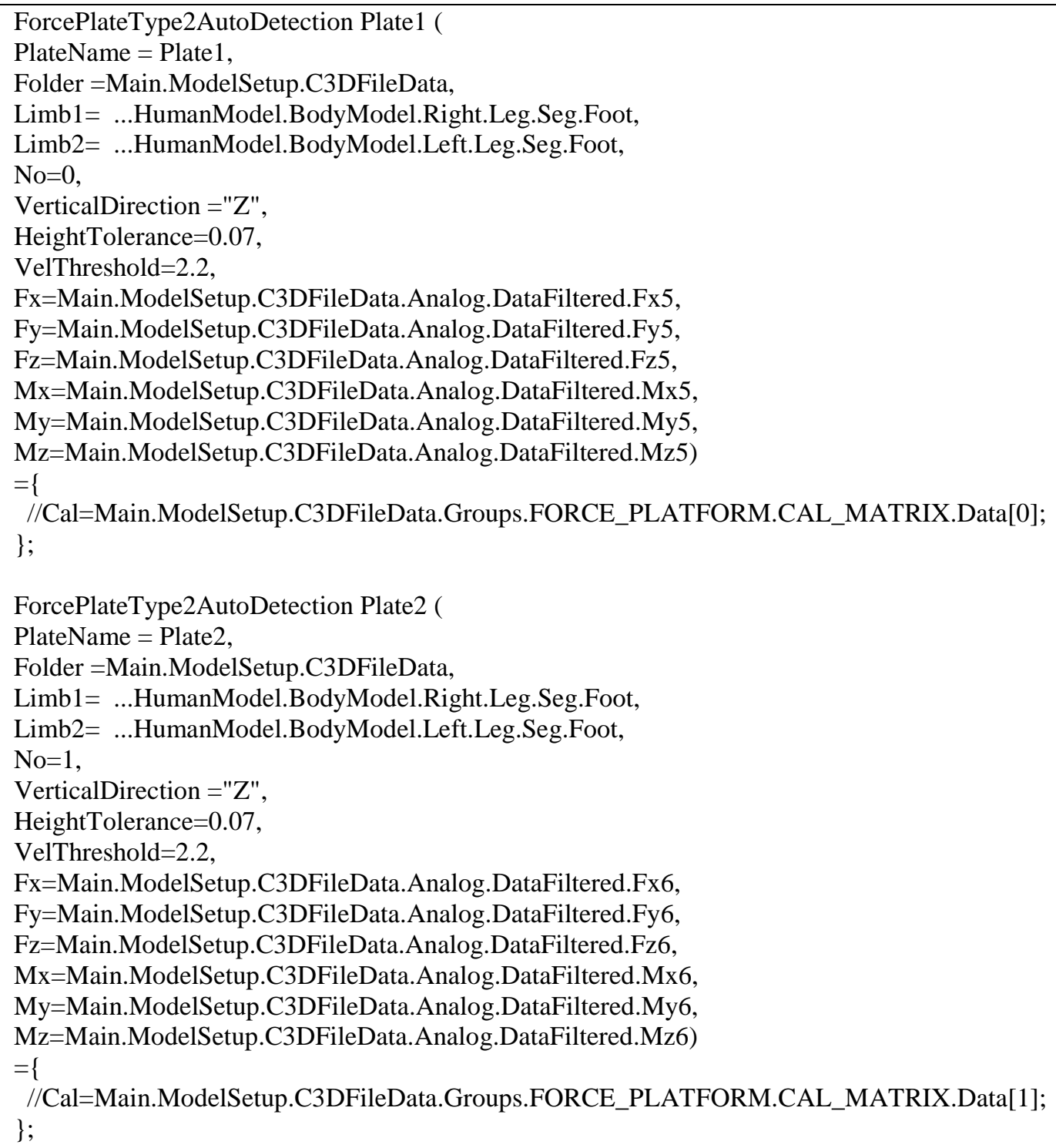

Figure 4.23: Model statement for auto detection of force plate touched by the foot steps

\subsubsection{4 "Joint Angle Outputs"}

The "Joint Angle Outputs" file automatically saved the optimized motion in text files named after the C3D file name. For example, the File Name was "Subject name-20 kgpush-10 $10^{\circ}$-Trial 2-output-euler-trunk.txt." Based on the existing setup for saving the optimized 
motion, an output file for the cart motion was created so that the cart motion data was dumped out automatically for later inverse dynamic study use.

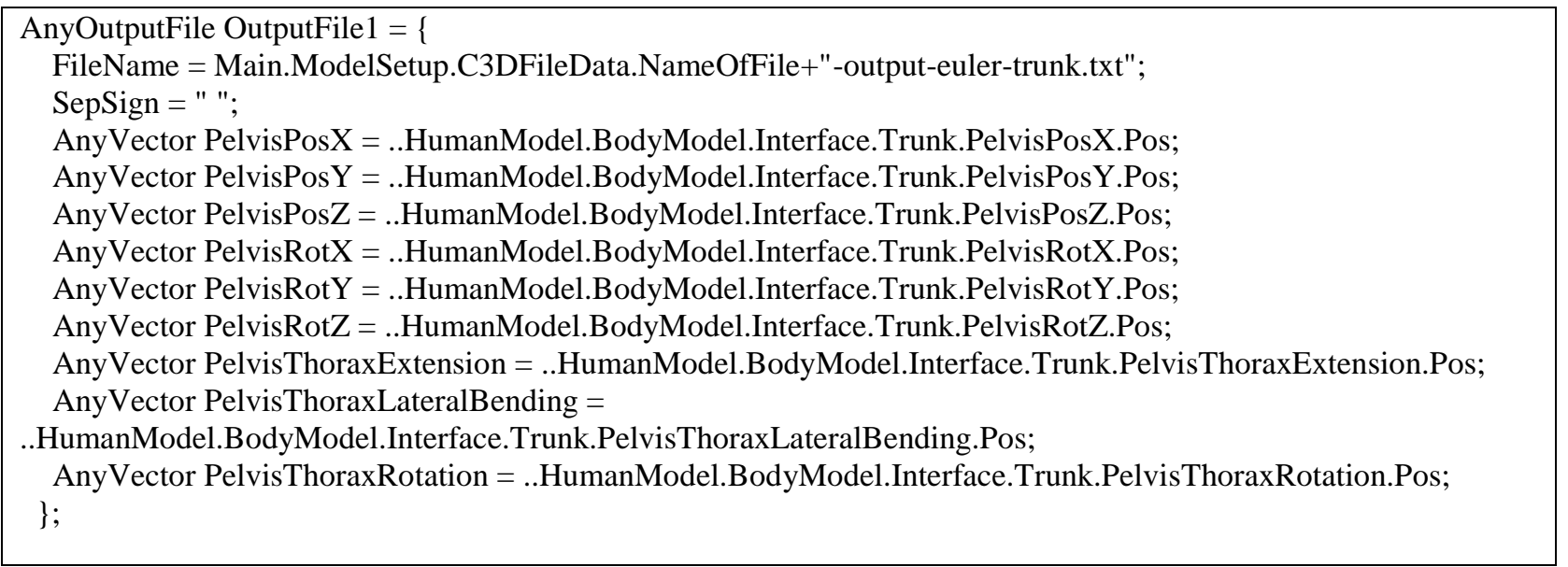

Figure 4.24: Model statement for automatic saving the motion data of the trunk

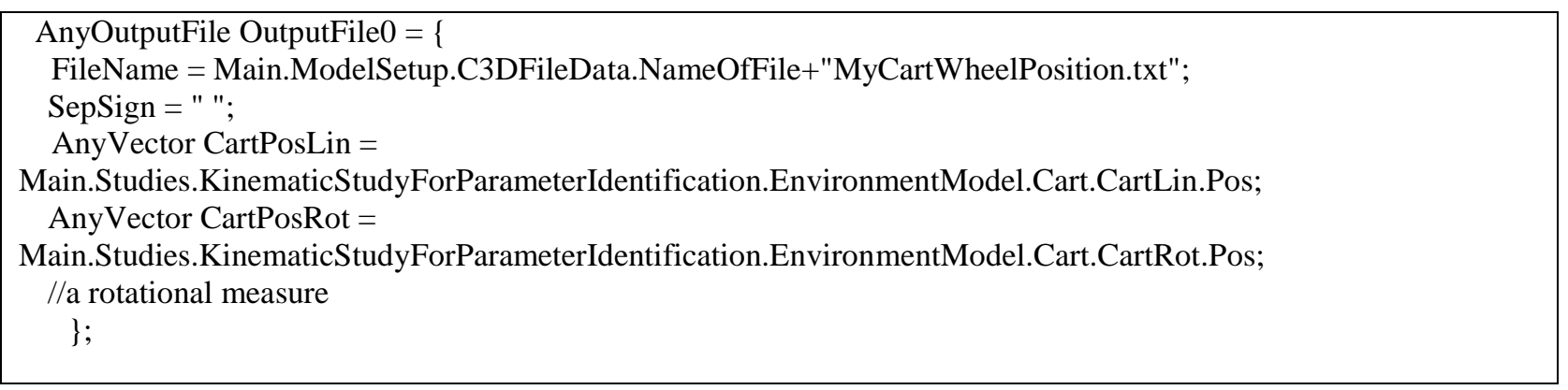

Figure 4.25: Model statement for automatic saving the cart motion data

\subsubsection{5 "Joints and Drivers Optimized"}

The "Joints and Drivers Optimized" file was used to automatically run the optimized motion in text files, which are already saved in the previous kinematic optimization study. Based on the existing file, a driver for the cart was created to control the motion of the cart in the inverse dynamic study with the use of "AnyKinEqInerPolDriver" term. This driver would read directly from the already saved text file.

\footnotetext{
AnyKinEqInterPolDriver JntDriverTrunk $=\{$

FileErrorContinueOnOff = On;

Type $=$ Bspline;

BsplineOrder $=4$;

FileName = Main.ModelSetup.C3DFileData.NameOfFile+"-output-euler-trunk.txt";

AnyKinMeasureOrg \&PelvisPosX = ...HumanModel.Interface.Trunk.PelvisPosX;

AnyKinMeasureOrg \&PelvisPosY $=\ldots$ HumanModel.Interface.Trunk.PelvisPosY;
} 
AnyKinMeasureOrg \&PelvisPosZ = ...HumanModel.Interface.Trunk.PelvisPosZ;

AnyKinMeasureOrg \&PelvisRotX = ...HumanModel.Interface.Trunk.PelvisRotX;

AnyKinMeasureOrg \&PelvisRotY = ...HumanModel.Interface.Trunk.PelvisRotY;

AnyKinMeasureOrg \&PelvisRotZ = ...HumanModel.Interface.Trunk.PelvisRotZ;

AnyKinMeasureOrg \&PelvisThoraxExtension $=\ldots$ HumanModel.Interface.Trunk.PelvisThoraxExtension;

AnyKinMeasureOrg \&PelvisThoraxLateralBending =

...HumanModel.Interface.Trunk.PelvisThoraxLateralBending;

AnyKinMeasureOrg \&PelvisThoraxRotation $=$...HumanModel.Interface.Trunk.PelvisThoraxRotation;

Reaction. Type $=\{$ On,On,On,On,On,On,Off,Off,Off $\}$;

\}

Figure 4.26: Model statement for running the optimized trunk motion data in inverse dynamic analysis step

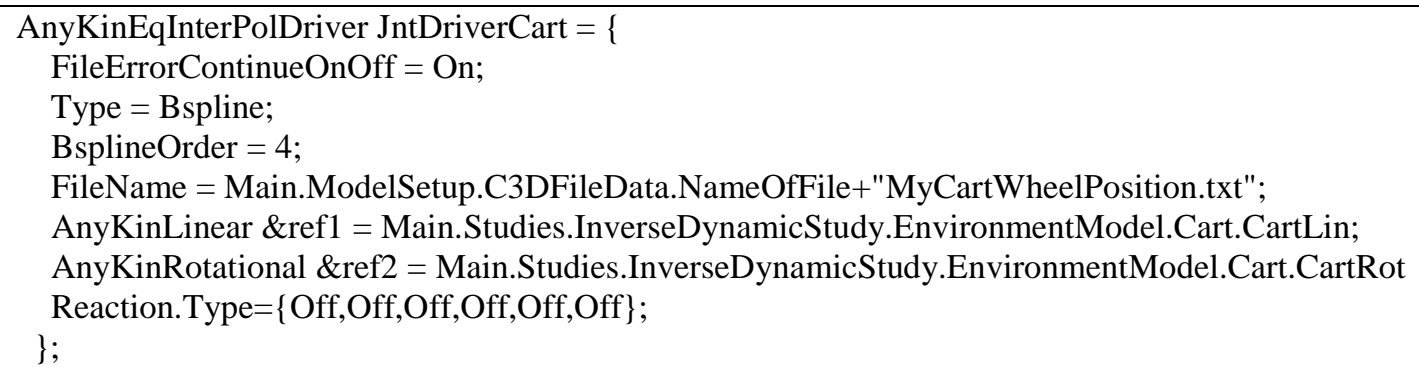

Figure 4.27: Model statement for running the optimized cart motion data in inverse dynamic analysis step

\subsubsection{Running inverse dynamic study}

Upon finishing all the aforementioned modeling steps, the system performed an actual inverse dynamic analysis based on the parameters identified in the previous MotionAndParameterOptimization step. This operation loaded the optimized model parameters that were saved previously; if necessary, it would perform calibration movements to adjust the tendon lengths to the lengths of the bones; and finally it executed an inverse dynamic analysis to determine forces in the system, as shown in Figure 4.19. When the analysis was complete, the ChartFX view would demonstrate all the biomechanical loading and muscle forces, and so on. The joint reaction forces at the $\mathrm{LSS1}, \mathrm{SC}, \mathrm{AC}$, and $\mathrm{GH}$ joint were dependent variables in the present study. The output was exported into a series of data sheets for later statistical analysis. 


\subsection{Statistical Analysis}

Minitab version 15 (Minitab Inc., PA, USA) was used to manage and analyze data in the current study. A general linear model with ANOVA (analysis of variance) was used to determine the effect of independent variables on the response variables.

In the first session where participants pushed the cart by varying the load weight along the flat walkway, the load weight was the single independent variable, which was treated as a fixed factor at three levels, while the subject was treated as a random factor. No interaction was evaluated in this simple general linear model (Model 1).

In session two where participants pushed or pulled the cart along the ramps by varying the load weight, walkway gradients and direction of force application along the ramps, there were three fixed factors: load weight (at three levels), walkway gradient (at two levels), and direction of force application (at two levels). Subjects were also treated as random factors in this model (Model 2). There were several two-way interaction factors (walkway gradient $\times$ load weight, walkway gradient $\times$ direction of force application, and load weight $\times$ direction of force application) and one three-way interaction factor (walkway gradient $\times$ load weight $\times$ direction of force application) included in the general linear model.

For both sessions, a post hoc trend analysis was performed using Tukey's HSD (Honestly Significant Differences) test to make All pair-wise comparisons (Appendix K). The significance level was set at $5 \%$.

It was assumed that the random error of the ANOVA models were normally, identically,

and independently NID $\left(0, \sigma^{2}\right)$ distributed. Minitab can also generate residual plots to examine how well the linear model fit. Therefore, a normal probability plot of residuals and a histogram of residuals were used to test the assumption: error $\epsilon \sim \operatorname{NID}\left(0, \sigma^{2}\right)$. 

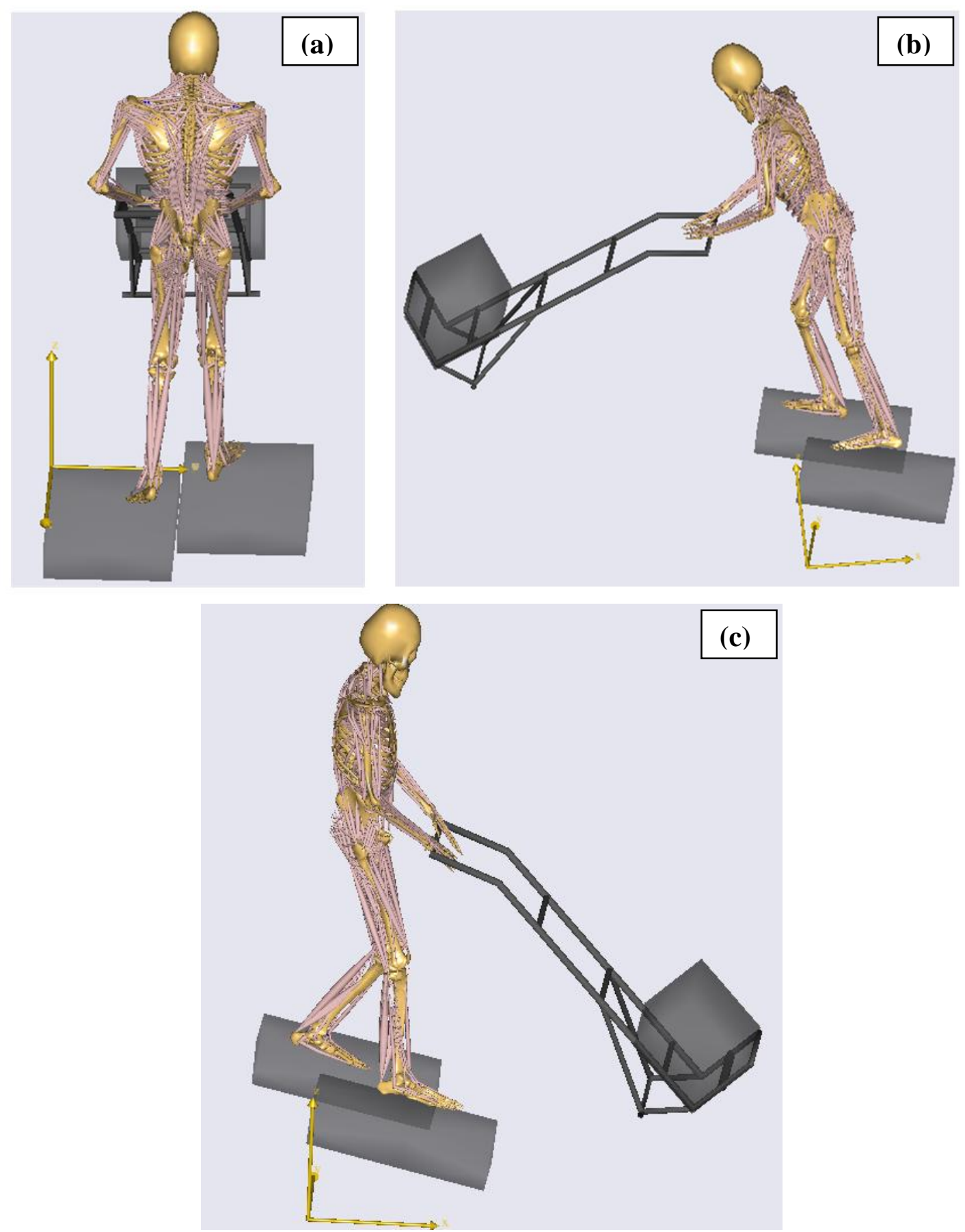

Figure 4.28: The biomechanical model used for quantifying the musculoskeletal loading created by the AnyBody modeling system: (a) pushing along the flat walkway; (b) uphill pushing along a ramp; (c) downhill pulling along a ramp 


\section{CHAPTER 5: MODEL VALIDATION}

\subsection{Introduction}

Validating a musculoskeletal model is necessary. Muscle force patterns are typically compared to electromyography (EMG) activity patterns to validate the results of the musculoskeletal model (Erdemir, et al., 2007). For example, to validate an upper extremity musculoskeletal model, Morrow et al. (2010) and Dubowsky et al. (2008) compared the simulation results with the experimental EMG data for each subject during wheelchair activities using the mean absolute error between the EMG activity envelope and the predicted muscle activity from the model. In the study of Marras et al. (1991c), a three-dimensional EMG-assisted model was validated by comparing the calculated trunk torque with measured trunk torque during dynamic lifting exertions. Plamondon et al. (1996) examined the validity of two threedimensional segment models to estimate the net reaction forces and moments at the L5S1 joint. By applying the models to lifting activities, results from the two models in terms of joint reaction forces and joint reaction moments at the L5S1 joint were compared between the two models. In a different study, De looze et al. (1992) used ground reaction force data to validated a twodimensional dynamic linked segment model. The reaction forces predicted by the model were compared with measured vertical ground reaction forces during lifting activities to validate the biomechanical model. Similar to the study of De looze et al. (1992), the model output of cart wheel reaction forces in the current study were compared to experimentally recorded ground reaction force $(\mathrm{GRF})$ data by the force plates to validate the modeling procedure.

\subsection{Approach}

The force equilibrium in the AnyBody modeling system is determined by the muscles and the reaction forces in the model. The accuracy of the computed cart wheel reaction forces will affect the output of muscle forces and joint reaction forces in the process of inverse dynamic analysis. Therefore, it is important to validate the accuracy of the predicted cart wheel reaction forces.

In this study, the ground reaction force (GRF) was recorded by the force plates at two instances: (1) when the cart wheel is moving over the force plates; (2) when the participant is 
stepping on the force plates. The first part data will be used to conduct the validation. Since the experimental data is being used for running the musculoskeletal model, the part of the experimental data when the cart wheel is moving over the force plate needs to be zeroed. GRF platform measured cart wheel forces in three dimensions (Fx', Fy', Fz'). The Anybody musculoskeletal model used in this study output only the forces in the vertical direction (Fz). Therefore, the two vertical forces $\left(\mathrm{Fz}^{\prime}\right.$ and $\left.\mathrm{Fz}\right)$ for the cart wheel were compared to validate the musculoskeletal model in this study.

On average, the duration for each cart wheel moving over the force plate was $0.6 \pm 0.10$ seconds. The minimum duration was approximately 0.5 seconds. The analog GRF data was recorded at a sampling rate of $100 \mathrm{~Hz}$, meaning that 100 frames are equal to 1 second. Therefore GRF data corresponding to 50 frames from the C3D file were used as the reference for making the comparison. In the AnyBody modeling system, the start time was matched to the starting frame of the 50 chosen frames. For example, the 50 frames (from frame number 101 to frame number 150) were chosen as a reference in the experimentally recorded GRF data. After zeroing the load weight, the trial in C3D file format was loaded into the AnyBody modeling system. The start time was matched to the beginning frame number, by using "AnyFloatVar tStart = 101/Main.ModelSetup.C3DFileData.Header.VideoFrameRate." The ending time of the AnyBody file was also matched to the ending frame, by using "AnyFloatVar tEnd = 150/Main.ModelSetup.C3DFileData.Header.VideoFrameRate." When the cart wheel reaction forces data were exported from the AnyBody output, the first time column followed by the column of reaction forces data was shown in seconds from 1.01 to 1.50 seconds. Validity (accuracy) was then evaluated by determining the difference between the values during the same time period (time $=0.5$ seconds) computed by the AnyBody modeling system and each frame of 50 frames obtained by the optical motion analysis system. 


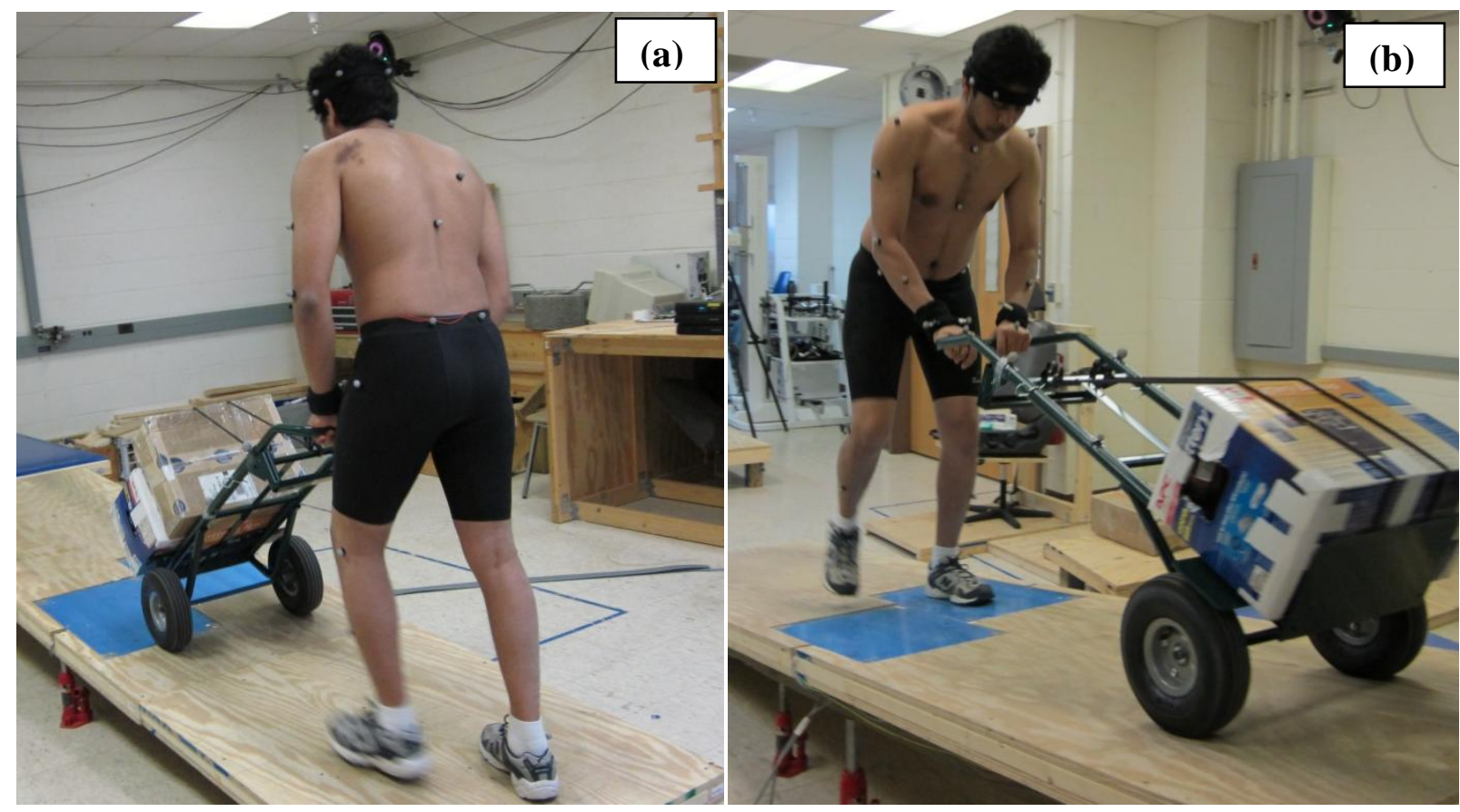

Figure 5.1: (a) Cart wheel is moving over the force plates; (b) the participant is stepping on the force plates

\subsection{Methods}

The original pipelined C3D data files from the optical motion analysis system were used as a reference to validate the accuracy of the simulated wheels' vertical forces by the AnyBody modeling system.

The process used to conduct the model validation consists of following steps:

(1) Kinematics data collection and processing:

In this step, marker and GRF data during pushing and pulling trials were recorded and processed using Vicon nexus software. The details of data collection and processing are mentioned in Methods chapter, Section 4.5 data collection procedures and Section 4.6.1 Kinematic data processing. Figure 5.1 (a) shows the two instances during which the GRF platform records the cart wheel forces and Figure 5.2 shows the processed kinematics data trial processed by the Vicon Nexus software. The two red arrows in the Figure 5.2 show the measured ground reaction forces. 
(2) Zeroing the cart wheel forces:

The C3Deditor (Motion Lab Systems, Inc., LA, USA), a graphical, C3D file editing program, was used to edit the $\mathrm{C} 3 \mathrm{D}$ files by zeroing the cart wheel forces. Figure 5.3 shows the distribution of ground reaction force (GRF) in the original C3D file output by the Vicon Nexus software when the participant pushed the load weight of $40 \mathrm{~kg}$ on the ramp of $5^{\circ} . \mathrm{In}$ the analog section of the original C3D file, the part of the frames reporting cart wheel GRF were identified, and the corresponding magnitudes were zeroed. Figure 5.4 shows the processed C3D file.

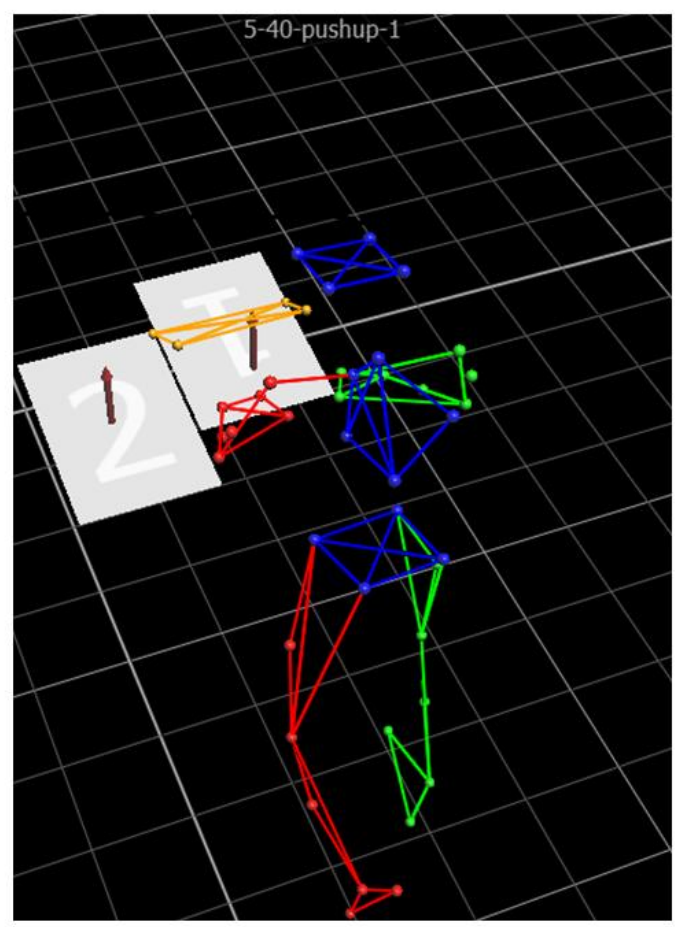

Figure 5.2: Kinematics data processed by the Vicon Nexus software, corresponding to the experimental trial shown in Figure 5.1(a) 


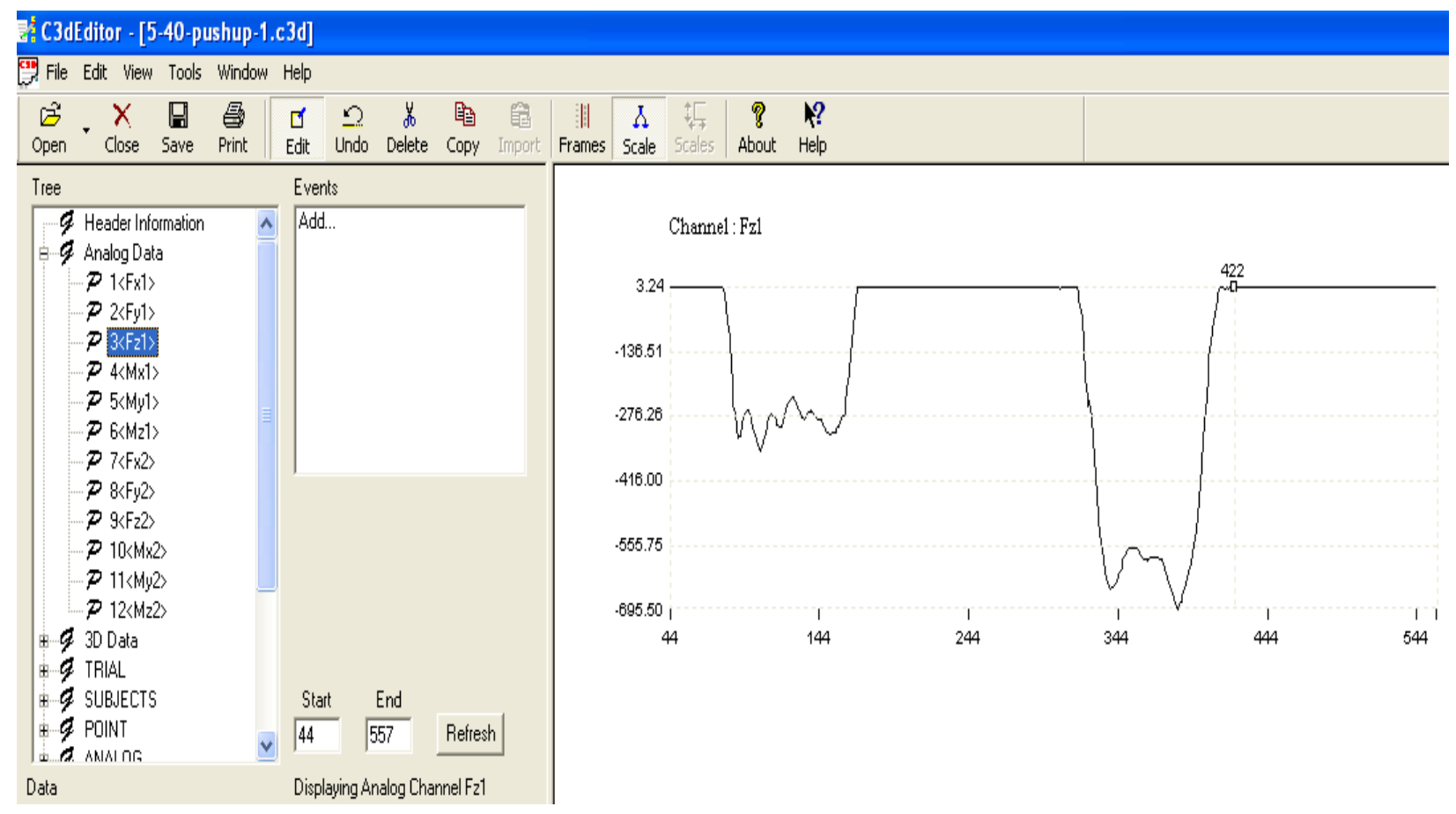

Figure 5.3: The originally stored Analog data of cart wheels and human body shown by C3Deditor in Windows

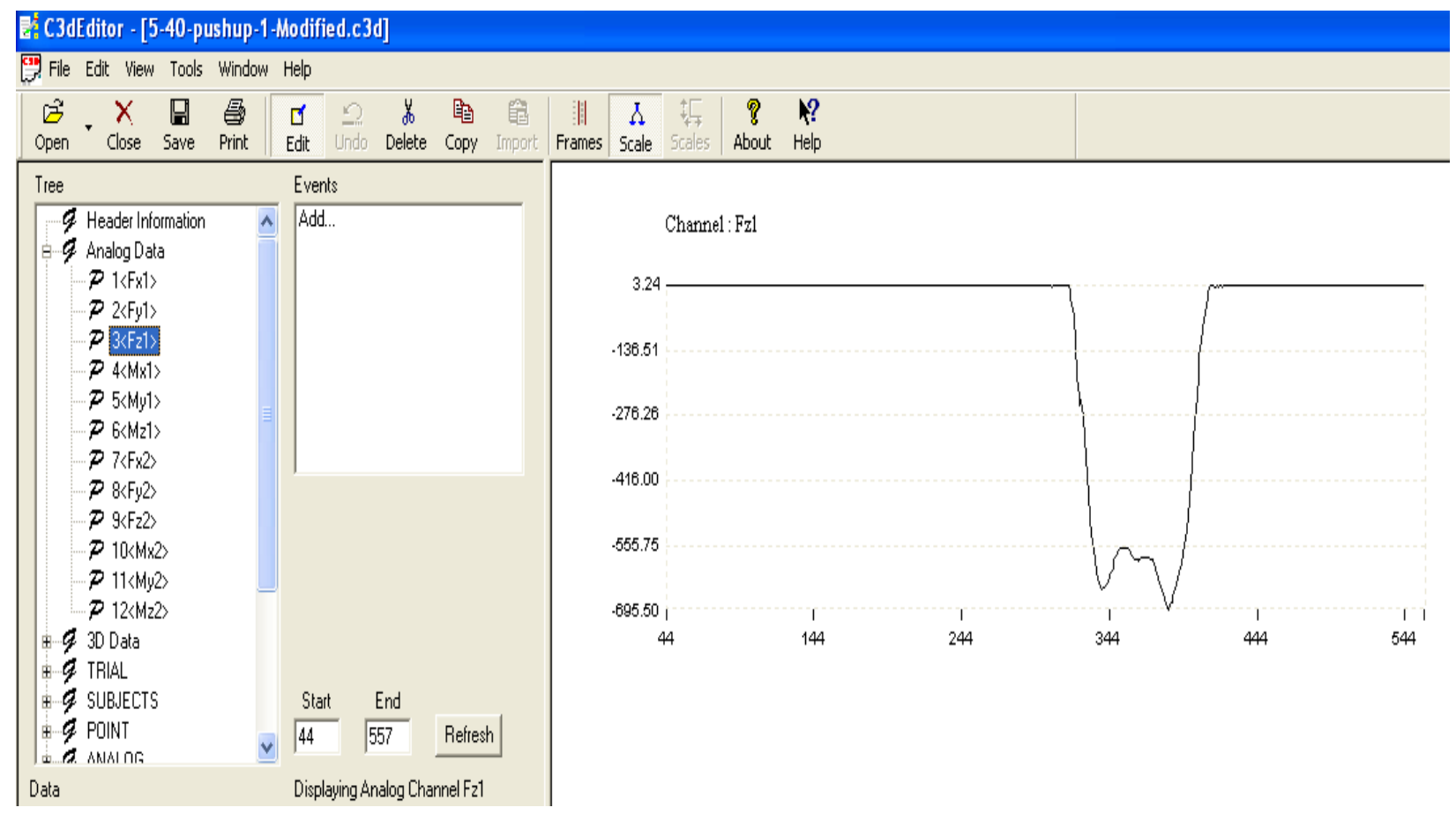

Figure 5.4: The modified Analog data after zeroing the cart wheel forces shown by C3Deditor in Windows 
(3) Model computations:

The modified C3D data were used to run the musculoskeletal model. Details of steps used to run the model are explained in the Methods chapter, Section 4.7.2. Inverse dynamic analysis was performed to compute vertical reaction forces for left and right cart wheels. Figure 5.5 shows the model statement used for calculating wheel forces, and Figure 5.6 shows a picture of the musculoskeletal model used to compute the cart wheels' vertical forces in the AnyBody modeling system platform.

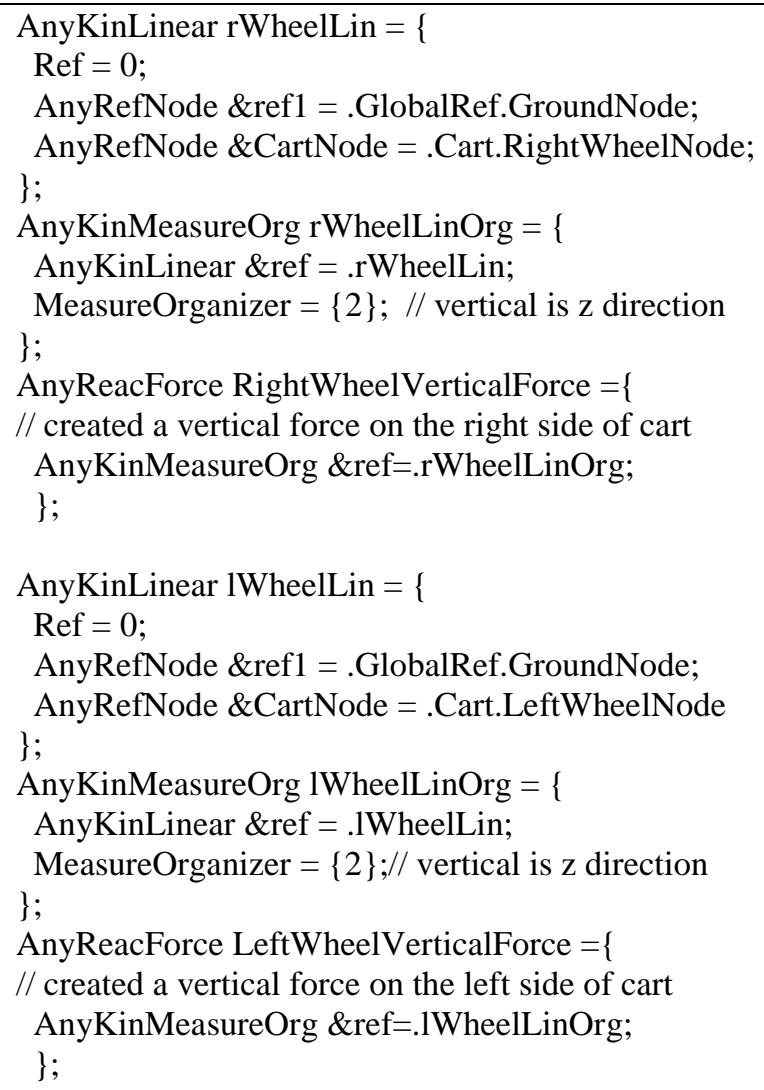

Figure 5.5: Model statement of computing cart wheels' vertical reaction forces

(4) Data processing:

Raw GRF data from force plates were low pass filtered by using a butter worth filter with the cutoff frequency of $5 \mathrm{~Hz}$. The forces during 50 frames (time $=0.5$ seconds) of each experimental condition were chosen to be compared predicted forces with the experimental data to test the accuracy of the musculoskeletal model. 


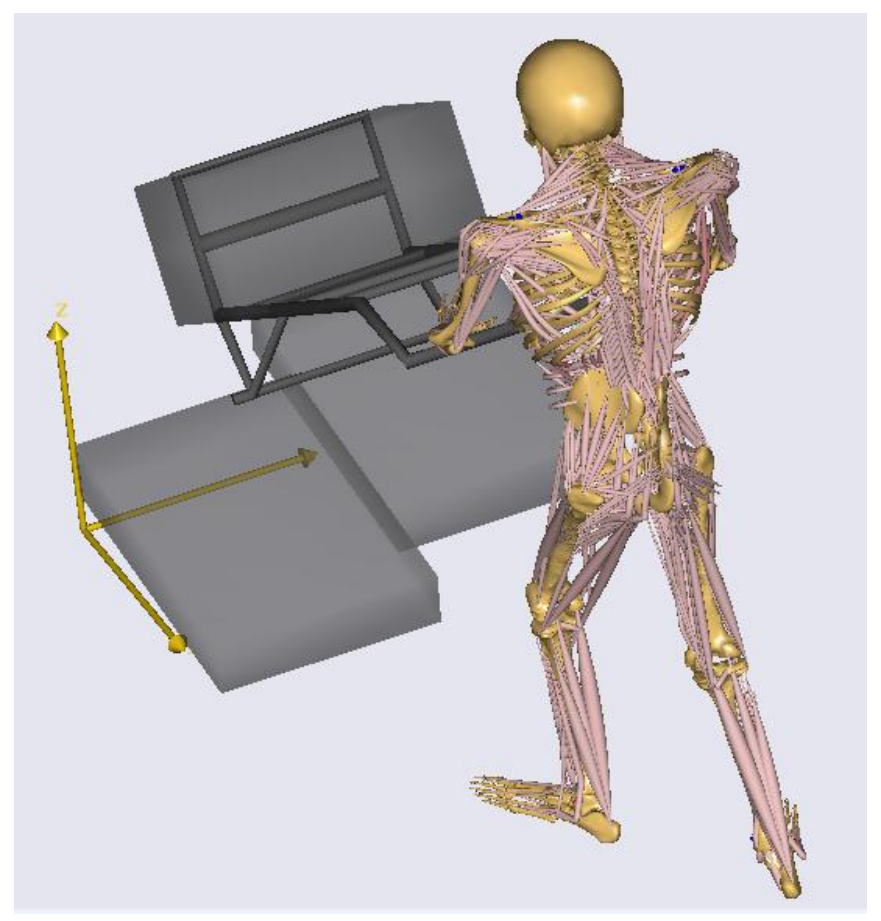

Figure 5.6: Picture of the musculoskeletal model used for computing cart wheels' vertical reaction forces

\subsection{Data analysis}

The data from six male participants (mean age $=23 \pm 4$ years; mean height $=1.70 \pm$ $0.2 \mathrm{~m}$; mean weight $=65.6 \pm 12.3 \mathrm{~kg}$ ) were used in this part of study to validate the accuracy of the musculoskeletal model. A procedure similar to Fong et al. (2008) was used for conducting the model validation computations. The vertical component of GRF ( $\left.\mathrm{Fz}^{\prime}\right)$ recorded by force plates was compared with the reaction force calculated by the model by using root mean squared error (RMSE) calculation (Fong, et al., 2008; Wong \& Wong, 2008; Plamondon, et al., 2007). All of the differences were converted to a RMSE using the following equation:

$$
R M S E=\left[\frac{1}{n} \sum_{i=1}^{n}\left(F z_{i}-F z_{i}^{\prime}\right)^{2}\right]^{1 / 2}
$$

Where,

$F z$ the vertical forces computed by AnyBody modeling system,

$F z^{\prime}$ the reference vertical forces measured by the GRF platform,

$n$ the number of samples ( $n=50$ frames), and $i$ the sample number $(i=1, \ldots, 50)$. 
In addition to the RMSE, the correlation coefficient (r) was also calculated to assess the accuracy of the computed wheel reaction forces using the following equation:

$$
r=\frac{\sum_{i=1}^{n}\left(F z_{i}-\overline{F z}\right)\left(F z_{i}^{\prime}-\overline{F z^{\prime}}\right)}{\sqrt{\sum_{i=1}^{n}\left(F z_{i}-\overline{F z}\right)^{2}} \sqrt{\sum_{i=1}^{n}\left(F z_{i}^{\prime}-\overline{F Z^{\prime}}\right)^{2}}}
$$

Where,

$F Z$ the vertical forces computed by AnyBody modeling system,

$F z^{\prime}$ the reference vertical forces measured by the GRF platform,

$n$ the number of samples ( $n=50$ frames), and $i$ the sample number $(i=1, \ldots, 50)$.

ANOVA tables (Appendix D) were provided to estimate the effect of load weight, walkway gradient, and direction of force application on the calculated RMSE and r.

\subsection{Results}

The minimum and maximum correlations between computed wheel reaction forces by AnyBody modeling system and the reference vertical forces measured by the GRF force plates were 0.837 and 0.987 respectively. On average, the correlation between the actual and estimated data was 0.919 and the average RMSE was $15.06 \mathrm{~N}$, which was about $3.96 \%$ of the peak value recorded in all experimental conditions $(380 \mathrm{~N})$. The maximum RMSE across the subjects was $24.98 \mathrm{~N}(6.57 \%$ of the peak value) and the minimum RMSE was $8.83 \mathrm{~N}(2.32 \%$ of the peak value) (Table 5.1).

When participants performed the cart pushing tasks along the flat walkway, the calculated $\mathrm{r}$ and RMSE were not different from one another with respect to the three weight conditions ( $\mathrm{p}=0.557$ for $\mathrm{r} ; \mathrm{p}=0.074$ for RMSE). During uphill pushing and downhill pulling tasks the walkway gradient was found to have a significant effect on the computed correlation coefficient $(\mathrm{r})(\mathrm{p}<0.001)$. The effect of load weight was found to be significant for the computed RMSE $(\mathrm{p}=0.005)$. The direction of force application also had a significant effect on the RMSE ( $p=0.008)$. In addition, there was a significant interaction effect of walkway gradient by direction of force application on the RMSE $(\mathrm{p}=0.016)$. 
Figures 5.7 - 5.11 show the pattern of the actual and computed wheel reaction forces for a selected number of trials and the corresponding absolute error. In general, the pattern of computed reaction forces matched very well with the actual data.

\subsection{Discussion and Conclusion}

In the current study, an existing comprehensive full-body musculoskeletal model was modified by redefinition of the marker protocol (adding four cart markers), adding force platforms, the lab coordinate system and most importantly, by creating a cart as a rigid segment in the environment of the model. Interaction of the cart with the human model and environment was established by setting contact forces between the hand and the cart handle and cart wheels' reaction forces. The reaction forces on the cart wheels contribute to the computation of the joint reaction and muscle forces in order to maintain the model equilibrium and therefore can be used for model validation purpose. Previously, De Looze et al. (1992) have used similar approach to validate a biomechanical model.

The results of the validation study show a high correlation between the actual and predicted cart wheel reaction forces (average $=0.919$ ). The mean RMSE was $15.06 \mathrm{~N}$, which was $3.96 \%$ of the peak value of $380 \mathrm{~N}$. In the previous studies RMSE up to $6 \%$ of the peak value was reported to be acceptable (Fong, et al., 2008). Therefore, it was concluded that the musculoskeletal model developed in this study was accurately predicting the reaction forces and consequently the muscle and joint loading that resulted from its interaction with the cart. 
Table 5.1: Accuracy of the computed left cart wheel's vertical forces as represented by the correlation coefficient $(r)$ and the root mean square error (RMSE), and the peak forces measured by the GRF platform

\begin{tabular}{|c|c|c|c|c|c|c|c|c|c|c|c|c|c|c|c|}
\hline \multirow{2}{*}{$\begin{array}{c}\text { Experimental } \\
\text { Conditions }\end{array}$} & \multicolumn{2}{|c|}{ Subject 1} & \multicolumn{2}{|c|}{ Subject 2} & \multicolumn{2}{|c|}{ Subject 3} & \multicolumn{2}{|c|}{ Subject 4} & \multicolumn{2}{|c|}{ Subject 5} & \multicolumn{2}{|c|}{ Subject 6} & \multicolumn{2}{|c|}{ All subjects } & \multirow{2}{*}{$\begin{array}{c}\text { Peak } \\
\text { force in all } \\
\text { trials }(\mathrm{N})\end{array}$} \\
\hline & $r$ & RMSE & $r$ & RMSE & $r$ & RMSE & $r$ & RMSE & $r$ & RMSE & $r$ & RMSE & $r$ & RMSE & \\
\hline $0^{\circ}-20 \mathrm{~kg}$ & 0.968 & 10.33 & 0.963 & 9.83 & 0.959 & 11.83 & 0.933 & 11.55 & 0.935 & 12.92 & 0.954 & 10.8 & 0.952 & 11.21 & 216 \\
\hline $0^{\circ}-30 \mathrm{~kg}$ & 0.975 & 8.83 & 0.967 & 9.67 & 0.981 & 11.16 & 0.952 & 14.69 & 0.864 & 15.88 & 0.969 & 9.81 & 0.951 & 11.67 & 319 \\
\hline $0^{\circ}-40 \mathrm{~kg}$ & 0.986 & 12.64 & 0.932 & 12.79 & 0.953 & 14.33 & 0.930 & 17.83 & 0.918 & 12.26 & 0.914 & 11.89 & 0.939 & 13.62 & 380 \\
\hline $5^{\circ}-20 \mathrm{~kg}$-up & 0.966 & 8.93 & 0.968 & 10.03 & 0.948 & 9.3 & 0.846 & 19.22 & 0.895 & 20.08 & 0.963 & 10.55 & 0.931 & 13.02 & 220 \\
\hline $5^{\circ}-30 \mathrm{~kg}$-up & 0.955 & 13.88 & 0.958 & 10.02 & 0.946 & 10.96 & 0.944 & 20.34 & 0.830 & 18.98 & 0.959 & 10.18 & 0.932 & 14.06 & 301 \\
\hline $5^{\circ}-40 \mathrm{~kg}$-up & 0.957 & 14.72 & 0.933 & 13.97 & 0.955 & 10.68 & 0.968 & 22.78 & 0.896 & 21.78 & 0.854 & 8.54 & 0.927 & 15.41 & 371 \\
\hline $5^{\circ}-20 \mathrm{~kg}$-down & 0.984 & 13.51 & 0.962 & 13.9 & 0.837 & 10.86 & 0.940 & 23.46 & 0.871 & 22.67 & 0.962 & 14.78 & 0.926 & 16.53 & 218 \\
\hline $5^{\circ}-30 \mathrm{~kg}$-down & 0.948 & 13.82 & 0.952 & 15.14 & 0.927 & 11.02 & 0.908 & 21.94 & 0.842 & 23.42 & 0.946 & 15.69 & 0.921 & 16.84 & 288 \\
\hline $5^{\circ}-40 \mathrm{~kg}$-down & 0.987 & 15.2 & 0.968 & 15.86 & 0.942 & 13.89 & 0.880 & 20.46 & 0.883 & 24.06 & 0.923 & 13.26 & 0.931 & 17.12 & 361 \\
\hline $10^{\circ}-20 \mathrm{~kg}$-up & 0.912 & 15.27 & 0.949 & 12.08 & 0.876 & 12.49 & 0.933 & 12.92 & 0.902 & 24.24 & 0.948 & 12.69 & 0.920 & 14.95 & 220 \\
\hline $10^{\circ}-30 \mathrm{~kg}$-up & 0.926 & 18.4 & 0.905 & 11 & 0.883 & 12.56 & 0.906 & 15.68 & 0.922 & 24.56 & 0.901 & 11.24 & 0.907 & 15.57 & 297 \\
\hline $10^{\circ}$-40kg-up & 0.961 & 17.33 & 0.938 & 15.21 & 0.863 & 14.27 & 0.845 & 19.9 & 0.852 & 24.24 & 0.841 & 13.87 & 0.883 & 17.47 & 354 \\
\hline $10^{\circ}-20 \mathrm{~kg}$-down & 0.901 & 16.46 & 0.880 & 8.31 & 0.922 & 14.03 & 0.834 & 18.68 & 0.891 & 22.35 & 0.889 & 7.92 & 0.886 & 14.63 & 220 \\
\hline $10^{\circ}-30 \mathrm{~kg}$-down & 0.863 & 15.2 & 0.947 & 11.62 & 0.914 & 12.39 & 0.900 & 17.08 & 0.840 & 24.98 & 0.928 & 15.23 & 0.899 & 16.08 & 267 \\
\hline $10^{\circ}-40 \mathrm{~kg}$-down & 0.900 & 18.4 & 0.946 & 14.02 & 0.845 & 13.93 & 0.847 & 19 & 0.895 & 23.67 & 0.869 & 17.01 & 0.884 & 17.67 & 352 \\
\hline All & 0.946 & 14.19 & 0.945 & 12.23 & 0.917 & 12.25 & 0.904 & 18.37 & 0.882 & 21.07 & 0.921 & 12.23 & 0.919 & 15.06 & 380 \\
\hline
\end{tabular}



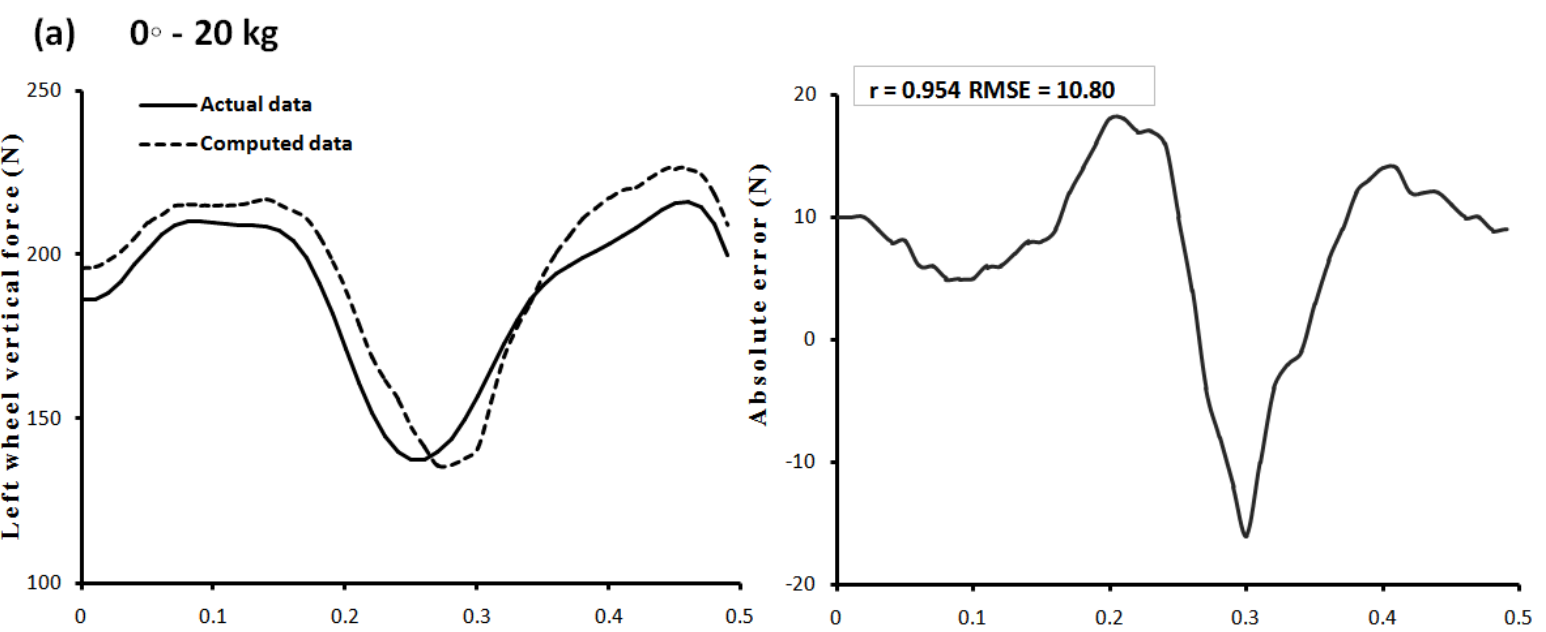

(b) $\quad 0 \circ-30 \mathrm{~kg}$
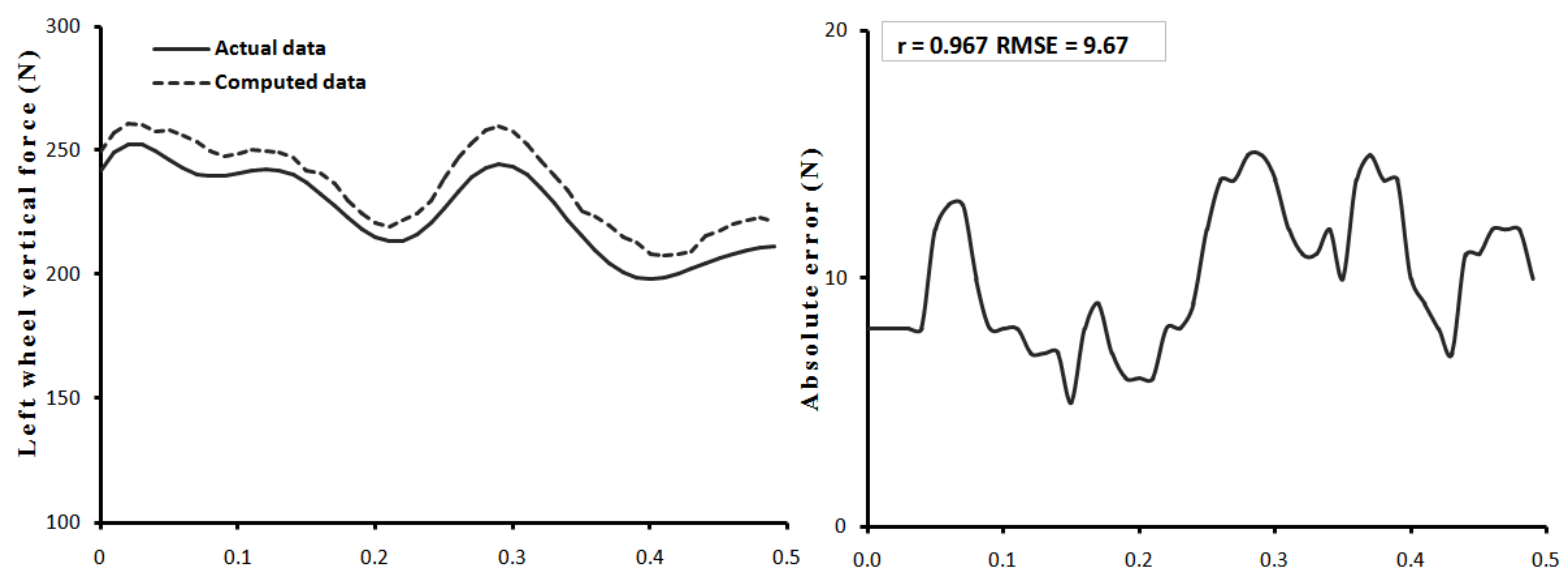

(c) $\quad 0-40 \mathrm{~kg}$
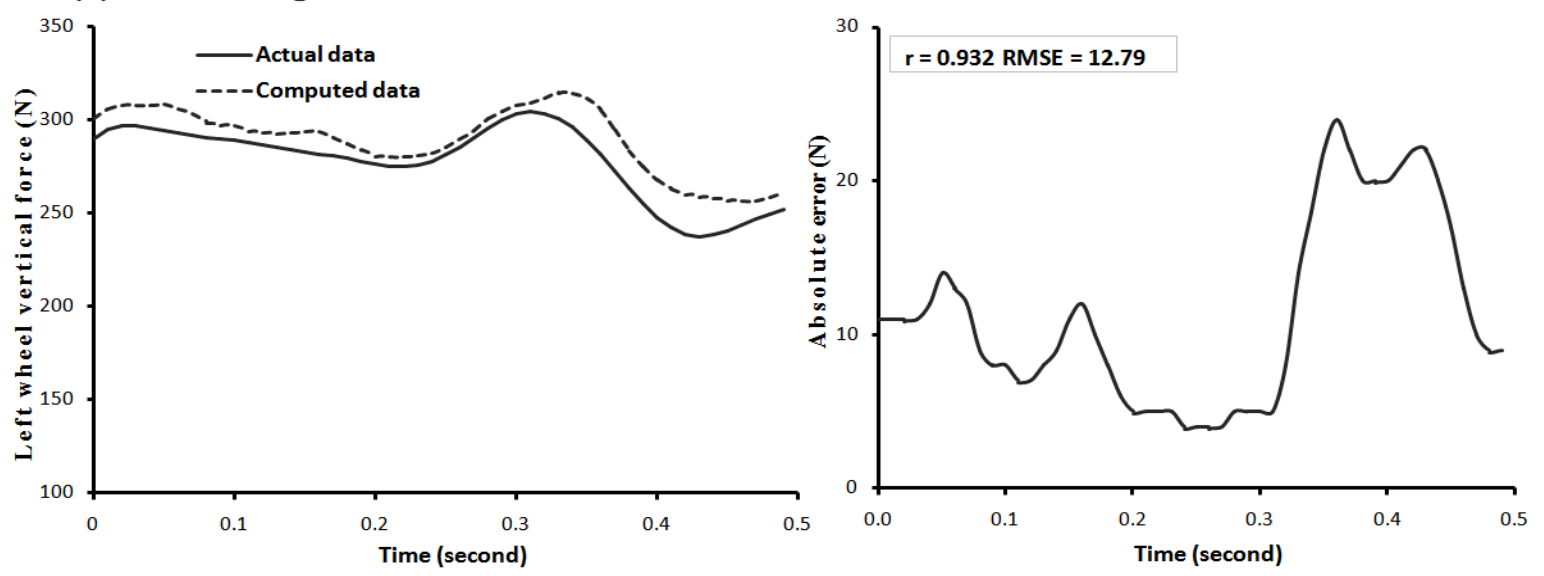

Figure 5.7: (a) - (c) The pattern of the actual and computed wheel reaction forces during pushing tasks performed at 20,30 , and $40 \mathrm{~kg}$ weight conditions on a flat walkway and the corresponding absolute error for averaged across the participants 

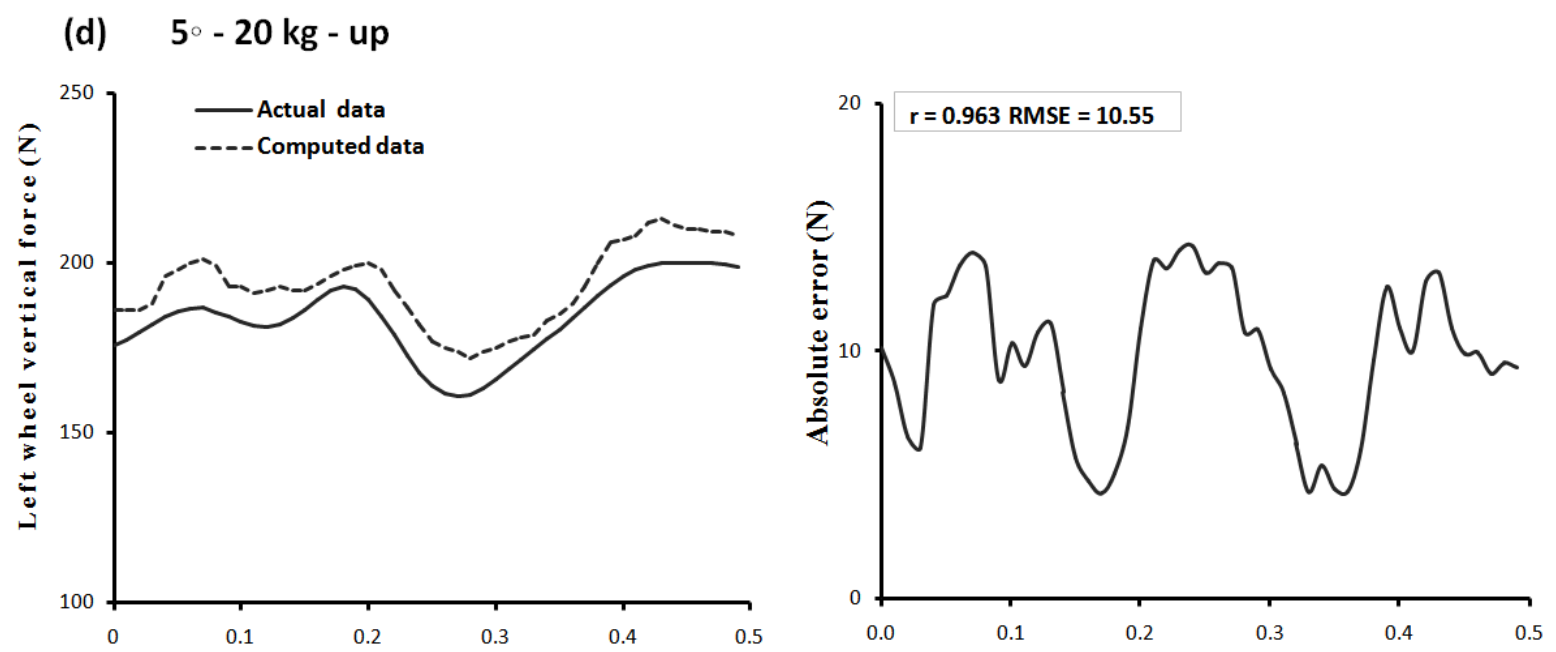

(e) $5 \circ-30 \mathrm{~kg}-$ up
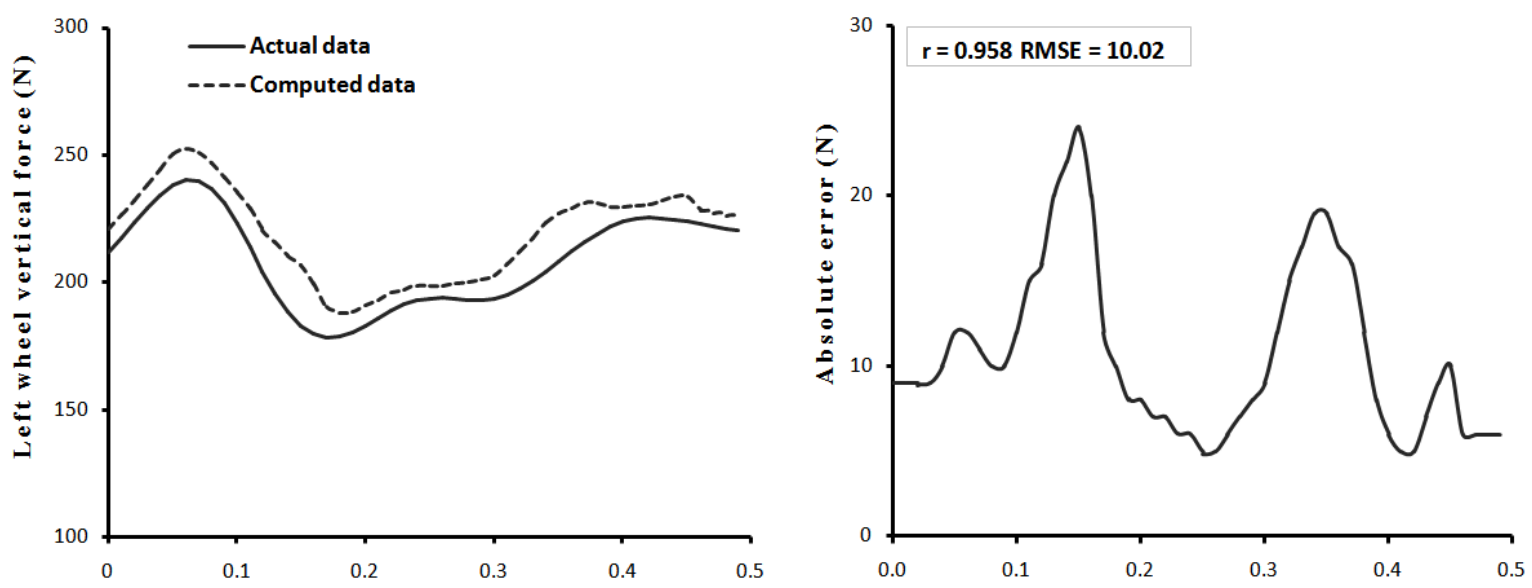

(f) 5 - $40 \mathrm{~kg}-$ up
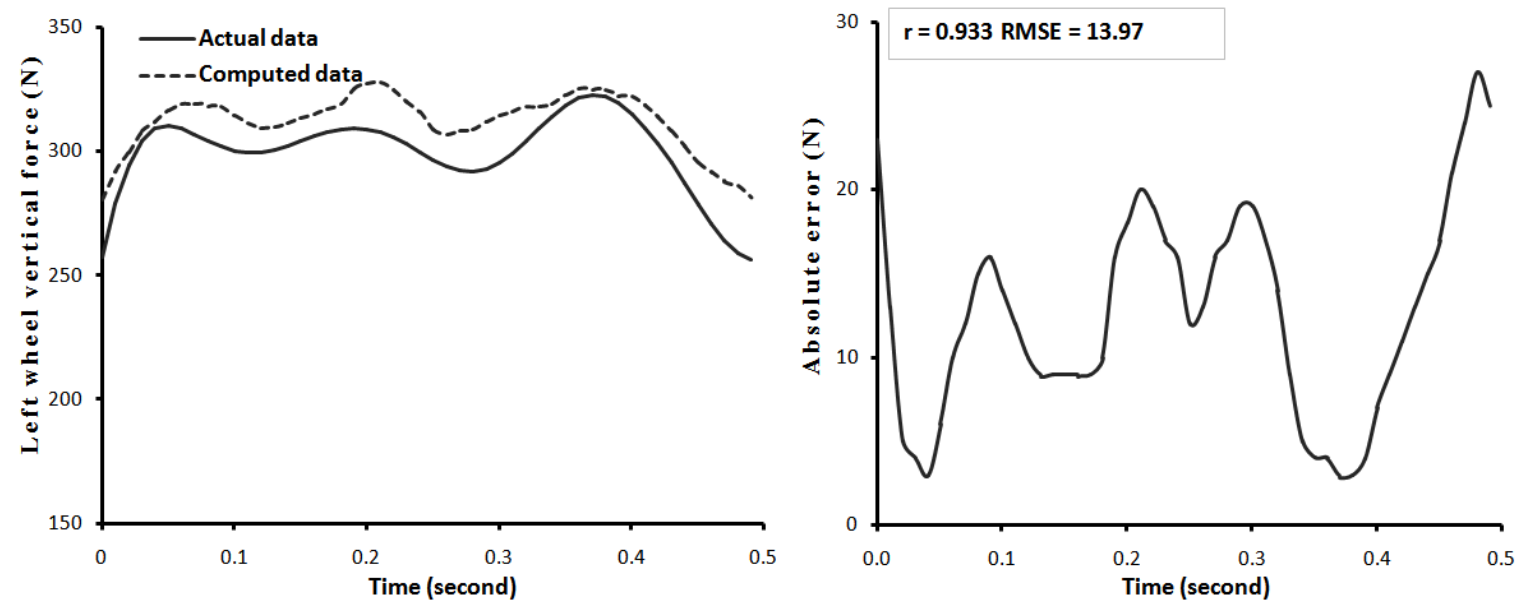

Figure 5.8: (d) - (f) The pattern of the actual and computed wheel reaction forces during uphill pushing tasks performed at 20,30 , and $40 \mathrm{~kg}$ weight conditions on a gradient of $5^{\circ}$ and the corresponding absolute error for averaged across the participants 

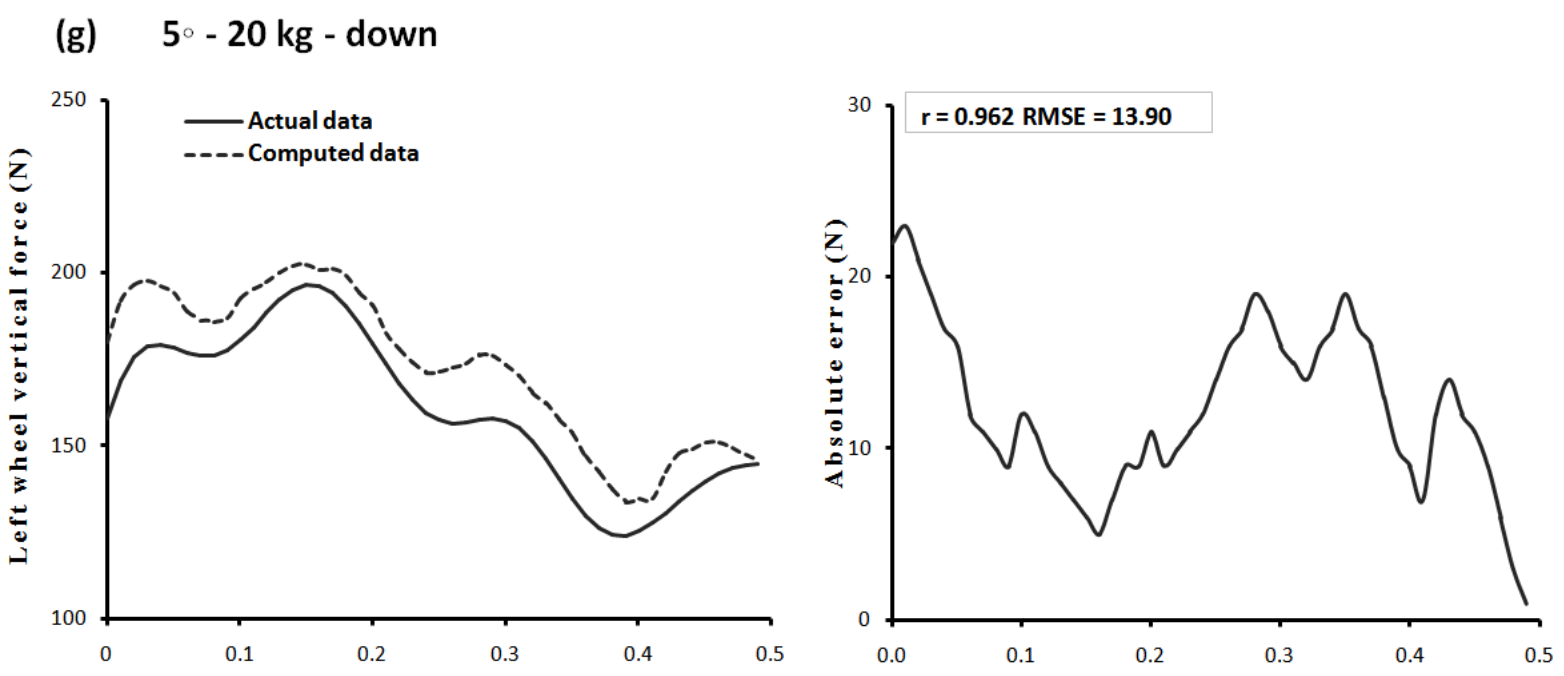

(h) $5 \circ-30 \mathrm{~kg}-$ down
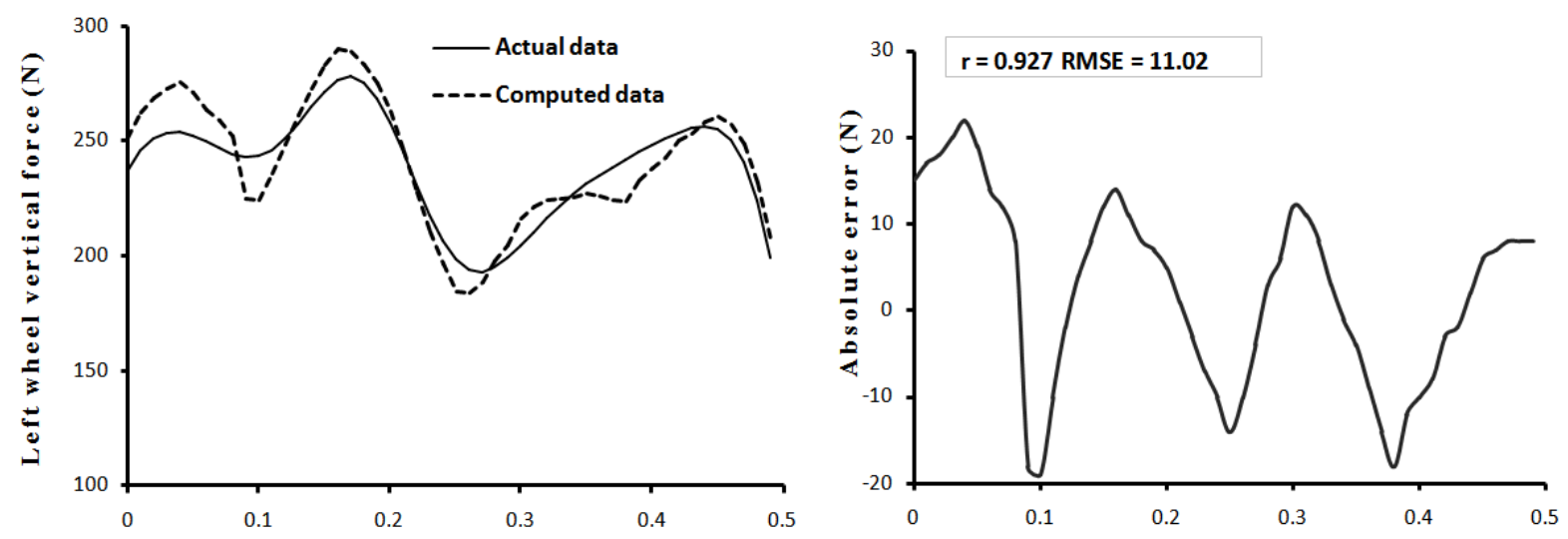

(i) $5-40 \mathrm{~kg}-$ down
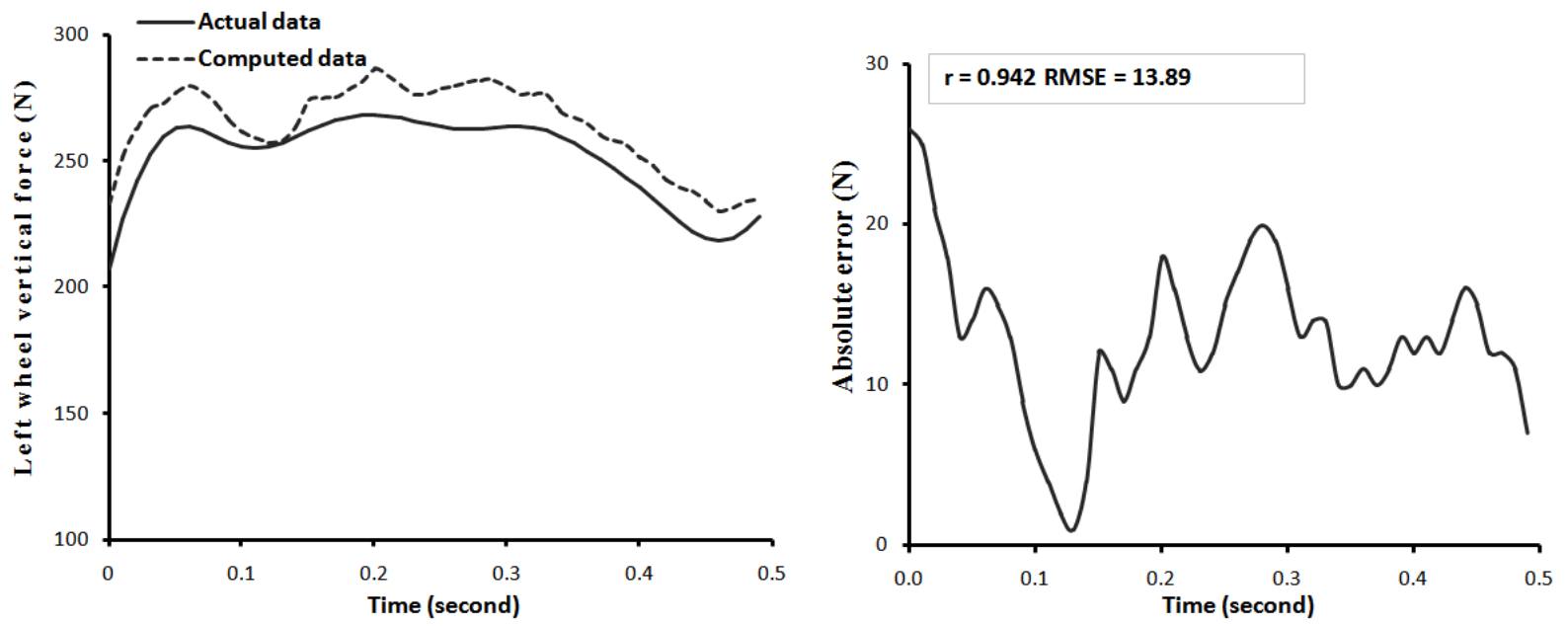

Figure 5.9: (g) - (i) The pattern of the actual and computed wheel reaction forces during downhill pulling tasks performed at 20,30 , and $40 \mathrm{~kg}$ weight conditions on a gradient of $5^{\circ}$ and the corresponding absolute error for averaged across the participants 

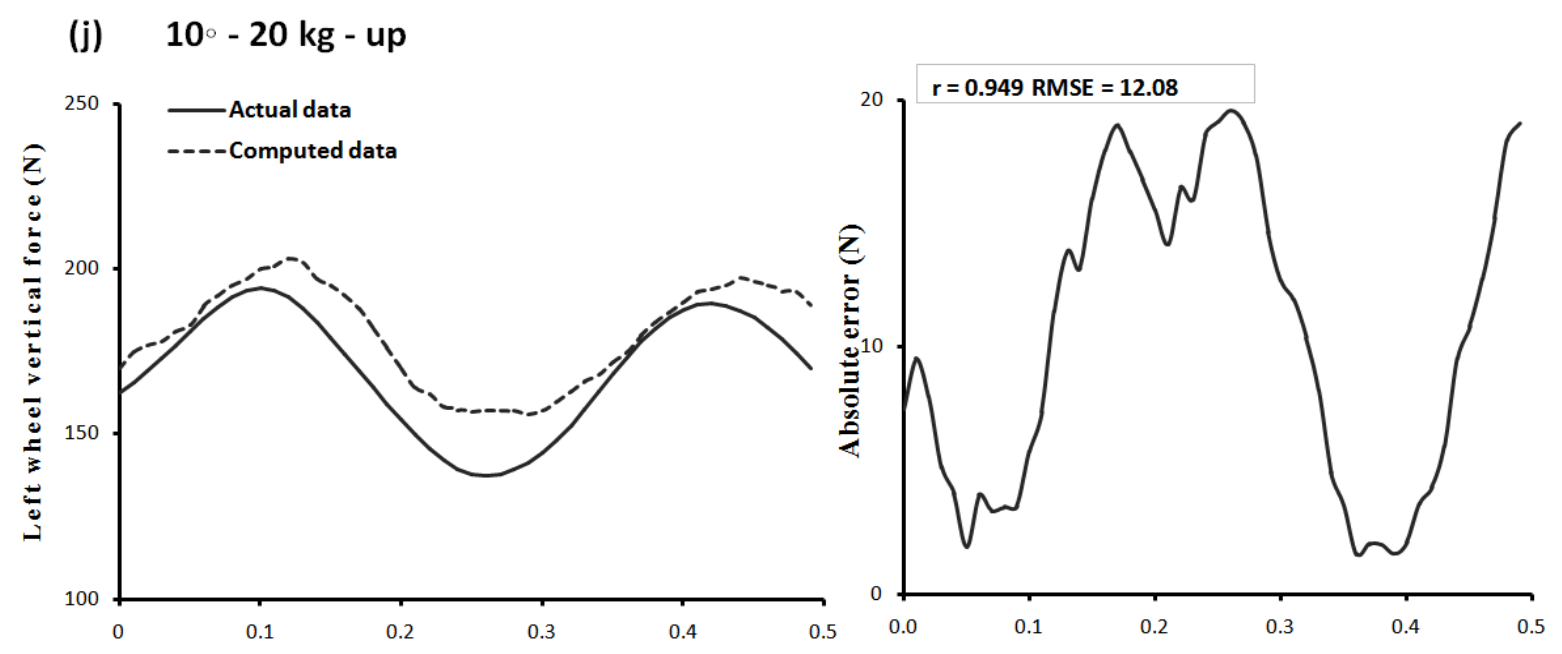

(k) $10-30 \mathrm{~kg}-$ up
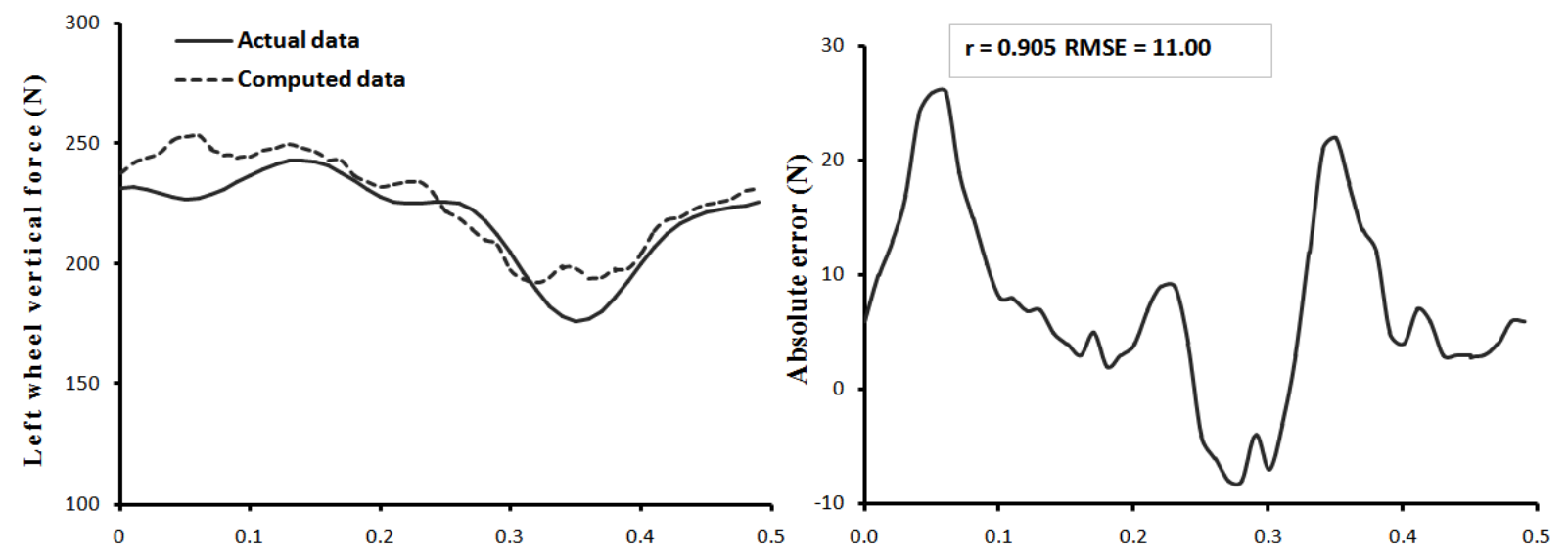

$$
\text { (I) } 10-40 \mathrm{~kg}-\text { up }
$$
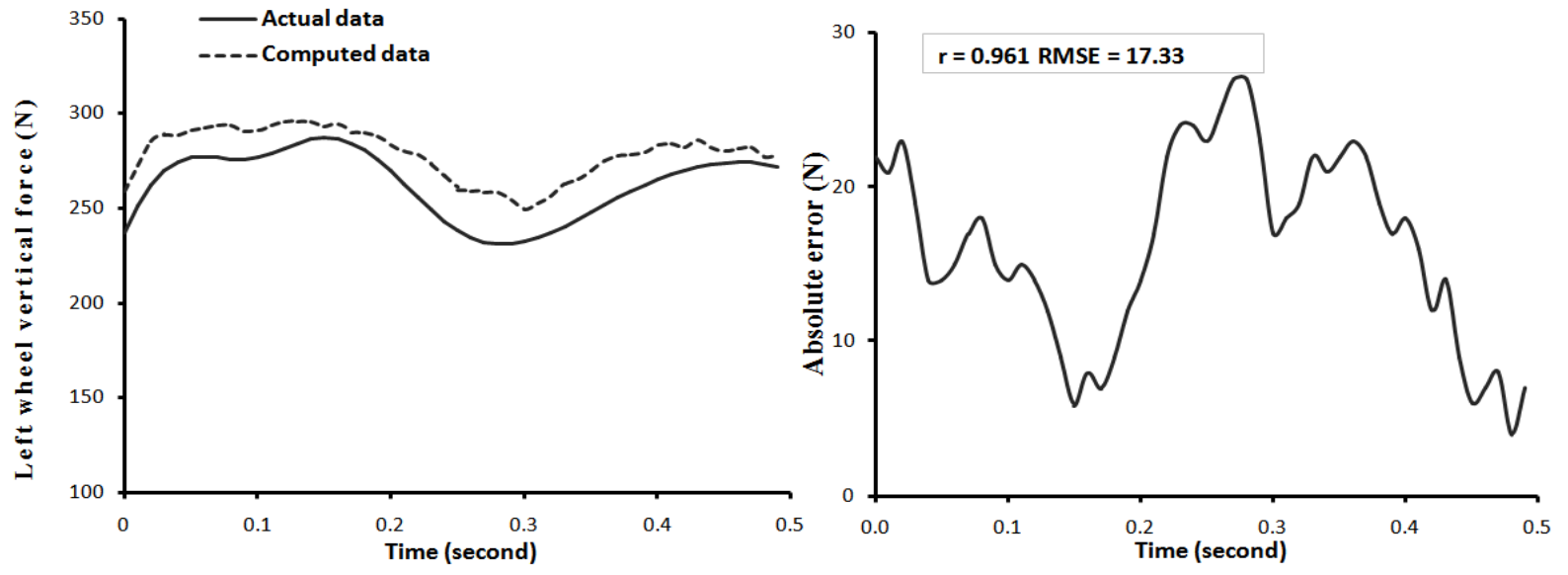

Figure 5.10: (j) - (1) The pattern of the actual and computed wheel reaction forces during uphill pushing tasks performed at 20,30 , and $40 \mathrm{~kg}$ weight conditions on a gradient of $10^{\circ}$ and the corresponding absolute error for averaged across the participants 

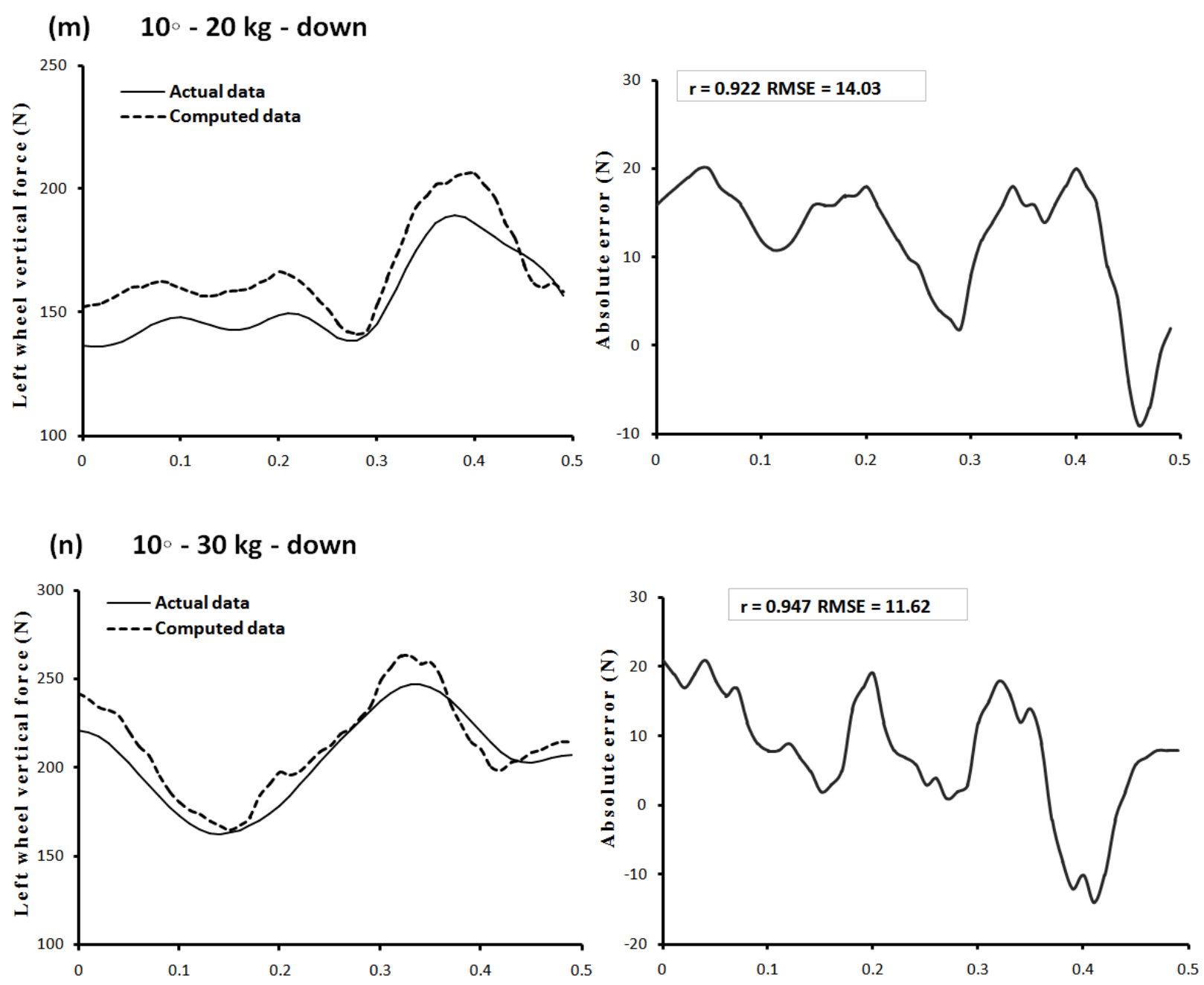

\section{(o) $10-40 \mathrm{~kg}-$ down}
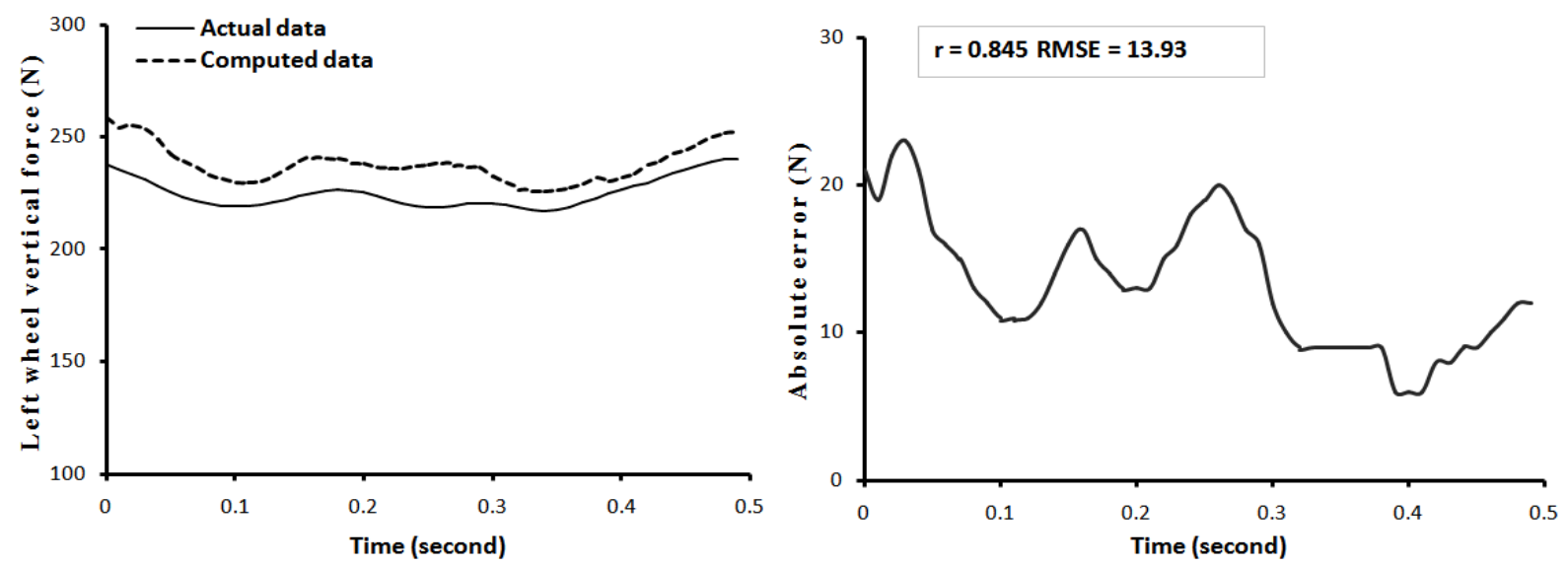

Figure 5.11: (m) - (o) The pattern of the actual and computed wheel reaction forces during downhill pulling tasks performed at 20,30 , and $40 \mathrm{~kg}$ weight conditions on a gradient of $10^{\circ}$ and the corresponding absolute error for averaged across the participants 


\section{CHAPTER 6: RESULTS}

\subsection{Subject Data}

Participants in this study were in the age group of 21 to 28 years. All the participants were males. A summary of participants' characteristics in terms of demographic data and anthropometric measurements is shown in Table 6.1. The data for individual participants can be found in Appendix E.

Table 6.1: Participants' demographic and anthropometric data

\begin{tabular}{|c|c|}
\hline & Mean (SD) \\
\hline Age (years) & $23.75(1.91)$ \\
\hline Weight $(\mathrm{kg})$ & $69.23(5.19)$ \\
\hline Height $(\mathrm{cm})$ & $172.04(4.44)$ \\
\hline Head height $(\mathrm{cm})$ & $17.41(0.99)$ \\
\hline Trunk height $(\mathrm{cm})$ & $49.93(3.75)$ \\
\hline Upper arm length $(\mathrm{cm})$ & $34.38(1.73)$ \\
\hline Lower arm length $(\mathrm{cm})$ & $27.34(2.01)$ \\
\hline Hand length $(\mathrm{cm})$ & $18.25(0.84)$ \\
\hline Hand breadth $(\mathrm{cm})$ & $8.40(0.58)$ \\
\hline Pelvis width $(\mathrm{cm})$ & $31.35(2.18)$ \\
\hline Thigh length $(\mathrm{cm})$ & $42.63(2.87)$ \\
\hline Shank length $(\mathrm{cm})$ & $41.28(2.31)$ \\
\hline Foot length $(\mathrm{cm})$ & $26.82(1.39)$ \\
\hline
\end{tabular}

\subsection{Experimental Data}

\subsubsection{Participants' ground reaction force (GRF) data}

Table 6.2 shows the measured peak ground reaction forces acted on the right and left foot across all experimental conditions. Raw GRF data are shown in Appendix F. 
Table 6.2: Mean (SD) of the peak ground reaction force (GRF) across all experimental conditions

\begin{tabular}{|c|c|c|c|c|c|c|c|c|c|c|c|c|}
\hline \multirow{2}{*}{$\begin{array}{c}\text { Experimental } \\
\text { Conditions }\end{array}$} & \multicolumn{6}{|c|}{ Right Foot } & \multicolumn{6}{|c|}{ Left Foot } \\
\hline & $\mathrm{Fx}$ & Fy & $\mathrm{Fz}$ & $\mathrm{Mx}$ & My & $\mathrm{Mz}$ & $\mathrm{Fx}$ & $\mathrm{Fy}$ & $\mathrm{Fz}$ & $\mathrm{Mx}$ & My & $\mathrm{Mz}$ \\
\hline $0^{\circ}-20 \mathrm{~kg}$ & $\begin{array}{c}56.8 \\
(11.4) \\
\end{array}$ & $\begin{array}{c}58.4 \\
(18.2) \\
\end{array}$ & $\begin{array}{l}686.8 \\
(48.7) \\
\end{array}$ & $\begin{array}{c}74.3 \\
(25.3) \\
\end{array}$ & $\begin{array}{c}43.5 \\
(13.6) \\
\end{array}$ & $\begin{array}{l}6.9 \\
(3)\end{array}$ & $\begin{array}{c}59.1 \\
(10.9)\end{array}$ & $\begin{array}{l}53.8 \\
(18) \\
\end{array}$ & $\begin{array}{l}676.9 \\
(56.8) \\
\end{array}$ & $\begin{array}{l}38.3 \\
(7.3) \\
\end{array}$ & $\begin{array}{l}35.4 \\
(9.3)\end{array}$ & $\begin{array}{c}5.3 \\
(1.8) \\
\end{array}$ \\
\hline $0^{\circ}-30 \mathrm{~kg}$ & $\begin{array}{c}55 \\
(8.6) \\
\end{array}$ & $\begin{array}{c}69.3 \\
(13.6) \\
\end{array}$ & $\begin{array}{l}681.3 \\
(45.7) \\
\end{array}$ & $\begin{array}{c}70.6 \\
(17.4) \\
\end{array}$ & $\begin{array}{l}40.4 \\
(8.5) \\
\end{array}$ & $\begin{array}{c}6.1 \\
(2.5) \\
\end{array}$ & $\begin{array}{l}56.4 \\
(7.9) \\
\end{array}$ & $\begin{array}{l}64.5 \\
(24) \\
\end{array}$ & $\begin{array}{l}672.5 \\
(45.1) \\
\end{array}$ & $\begin{array}{c}52.7 \\
(16.7) \\
\end{array}$ & $\begin{array}{c}35.3 \\
(10.9) \\
\end{array}$ & $\begin{array}{c}5.1 \\
(1.8) \\
\end{array}$ \\
\hline $0^{\circ}-40 \mathrm{~kg}$ & $\begin{array}{c}56.8 \\
(10.2) \\
\end{array}$ & $\begin{array}{l}83.9 \\
(16) \\
\end{array}$ & $\begin{array}{l}675.1 \\
(43.9) \\
\end{array}$ & $\begin{array}{c}73.2 \\
(25.2) \\
\end{array}$ & $\begin{array}{l}42.8 \\
(9.4) \\
\end{array}$ & $\begin{array}{c}5.4 \\
(2.7) \\
\end{array}$ & $\begin{array}{c}54.3 \\
(12.2) \\
\end{array}$ & $\begin{array}{c}76.8 \\
(28.8) \\
\end{array}$ & $\begin{array}{l}672.6 \\
(42.1) \\
\end{array}$ & $\begin{array}{c}68.7 \\
(26.9) \\
\end{array}$ & $\begin{array}{c}33.8 \\
(16.1) \\
\end{array}$ & $\begin{array}{c}6.1 \\
(2.3) \\
\end{array}$ \\
\hline $5^{\circ}-20 \mathrm{~kg}$-up & $\begin{array}{l}50.6 \\
(8.2) \\
\end{array}$ & $\begin{array}{l}135.4 \\
(23.1)\end{array}$ & $\begin{array}{c}667.8 \\
(45) \\
\end{array}$ & $\begin{array}{c}65.4 \\
(32.8) \\
\end{array}$ & $\begin{array}{c}40.5 \\
(11.4) \\
\end{array}$ & $\begin{array}{c}4.8 \\
(2.5) \\
\end{array}$ & $\begin{array}{c}60.2 \\
(15.5) \\
\end{array}$ & $\begin{array}{l}111.2 \\
(14.7) \\
\end{array}$ & $\begin{array}{l}674.4 \\
(48.2) \\
\end{array}$ & $\begin{array}{c}41.7 \\
(18.6) \\
\end{array}$ & $\begin{array}{c}36.8 \\
(10.7) \\
\end{array}$ & $\begin{array}{c}6.5 \\
(2.3) \\
\end{array}$ \\
\hline $5^{\circ}-30 \mathrm{~kg}$-up & $\begin{array}{l}56.7 \\
(14) \\
\end{array}$ & $\begin{array}{r}147.9 \\
(17.9) \\
\end{array}$ & $\begin{array}{l}673.2 \\
(38.7) \\
\end{array}$ & $\begin{array}{c}68.4 \\
(17.1) \\
\end{array}$ & $\begin{array}{c}44.2 \\
(13.5) \\
\end{array}$ & $\begin{array}{c}7.8 \\
(4.6) \\
\end{array}$ & $\begin{array}{l}59.5 \\
(7.6) \\
\end{array}$ & $\begin{array}{l}126.3 \\
(21.5) \\
\end{array}$ & $\begin{array}{c}653 \\
(18.9) \\
\end{array}$ & $\begin{array}{c}46.7 \\
(15.5) \\
\end{array}$ & $\begin{array}{c}37.7 \\
(10.9) \\
\end{array}$ & $\begin{array}{c}7.8 \\
(3.2) \\
\end{array}$ \\
\hline $5^{\circ}-40 \mathrm{~kg}$-up & $\begin{array}{c}56.1 \\
(11.3) \\
\end{array}$ & $\begin{array}{r}171.9 \\
(17.8) \\
\end{array}$ & $\begin{array}{l}666.5 \\
(65.7)\end{array}$ & $\begin{array}{c}60.8 \\
(24.8) \\
\end{array}$ & $\begin{array}{c}49.3 \\
(19.6) \\
\end{array}$ & $\begin{array}{c}9.2 \\
(5.8) \\
\end{array}$ & $\begin{array}{c}56.1 \\
(11.5)\end{array}$ & $\begin{array}{c}145 \\
(31.3) \\
\end{array}$ & $\begin{array}{l}678.7 \\
(51.9)\end{array}$ & $\begin{array}{c}44.3 \\
(18.9) \\
\end{array}$ & $\begin{array}{c}37.3 \\
(10.5)\end{array}$ & $\begin{array}{c}9.3 \\
(4.3) \\
\end{array}$ \\
\hline $5^{\circ}-20 \mathrm{~kg}$-down & $\begin{array}{c}72.3 \\
(17.9) \\
\end{array}$ & $\begin{array}{c}139.8 \\
(15.7) \\
\end{array}$ & $\begin{array}{c}740 \\
(45.1)\end{array}$ & $\begin{array}{c}54.6 \\
(14.9) \\
\end{array}$ & $\begin{array}{l}40.1 \\
(6.5) \\
\end{array}$ & $\begin{array}{c}7.7 \\
(2.9) \\
\end{array}$ & $\begin{array}{c}72.6 \\
(19.3) \\
\end{array}$ & $\begin{array}{l}168.4 \\
(25.8) \\
\end{array}$ & $\begin{array}{c}716 \\
(58.9) \\
\end{array}$ & $\begin{array}{c}45.6 \\
(17.2) \\
\end{array}$ & $\begin{array}{c}39.8 \\
(10.2) \\
\end{array}$ & $\begin{array}{l}8.6 \\
(4) \\
\end{array}$ \\
\hline $5^{\circ}-30 \mathrm{~kg}$-down & $\begin{array}{l}69.3 \\
(6.9) \\
\end{array}$ & $\begin{array}{l}133.6 \\
(28.8) \\
\end{array}$ & $\begin{array}{l}735.3 \\
(40.5) \\
\end{array}$ & $\begin{array}{c}68.3 \\
(14.6) \\
\end{array}$ & $\begin{array}{l}46.5 \\
(6.5) \\
\end{array}$ & $\begin{array}{l}10.3 \\
(2.8) \\
\end{array}$ & $\begin{array}{l}67.2 \\
(22) \\
\end{array}$ & $\begin{array}{l}162.8 \\
(38.6) \\
\end{array}$ & $\begin{array}{l}715.2 \\
(50.3) \\
\end{array}$ & $\begin{array}{c}52.2 \\
(22.8) \\
\end{array}$ & $\begin{array}{c}40 \\
(13.3)\end{array}$ & $\begin{array}{c}8.3 \\
(5.1) \\
\end{array}$ \\
\hline $5^{\circ}-40 \mathrm{~kg}$-down & $\begin{array}{r}70.6 \\
(8.6)\end{array}$ & $\begin{array}{l}146.8 \\
(28.5)\end{array}$ & $\begin{array}{l}726.2 \\
(73.5)\end{array}$ & $\begin{array}{c}70.6 \\
(14.3)\end{array}$ & $\begin{array}{c}43.4 \\
(13.8)\end{array}$ & $\begin{array}{c}10.8 \\
(5)\end{array}$ & $\begin{array}{c}69.5 \\
(22.8)\end{array}$ & $\begin{array}{l}173.3 \\
(47.3)\end{array}$ & $\begin{array}{c}728.4 \\
(59.7)\end{array}$ & $\begin{array}{c}68 \\
(57.3)\end{array}$ & $\begin{array}{l}36.8 \\
(9.2)\end{array}$ & $\begin{array}{c}9.8 \\
(1.8)\end{array}$ \\
\hline $10^{\circ}-20 \mathrm{~kg}$-up & $\begin{array}{c}49 \\
(9.9)\end{array}$ & $\begin{array}{c}196.4 \\
(14.4)\end{array}$ & $\begin{array}{r}649.9 \\
(37.3)\end{array}$ & $\begin{array}{l}54.3 \\
(21)\end{array}$ & $\begin{array}{l}46.8 \\
(7.7)\end{array}$ & $\begin{array}{c}15.4 \\
(5)\end{array}$ & $\begin{array}{c}62.9 \\
(14.2)\end{array}$ & $\begin{array}{c}184.1 \\
(19)\end{array}$ & $\begin{array}{c}671 \\
(67.9)\end{array}$ & $\begin{array}{c}52.9 \\
(22.3)\end{array}$ & $\begin{array}{c}33.3 \\
(13.8)\end{array}$ & $\begin{array}{l}11.8 \\
(6.4)\end{array}$ \\
\hline $10^{\circ}-30 \mathrm{~kg}$-up & $\begin{array}{l}47.8 \\
(12)\end{array}$ & $\begin{array}{c}209.7 \\
(10.2)\end{array}$ & $\begin{array}{l}650.5 \\
(51.9)\end{array}$ & $\begin{array}{c}44.6 \\
(24.1)\end{array}$ & $\begin{array}{l}47.8 \\
(8.5)\end{array}$ & $\begin{array}{l}16.7 \\
(5.5)\end{array}$ & $\begin{array}{l}62.9 \\
(10)\end{array}$ & $\begin{array}{c}191.8 \\
(18.1)\end{array}$ & $\begin{array}{l}657.8 \\
(66.4)\end{array}$ & $\begin{array}{c}49.8 \\
(22.4)\end{array}$ & $\begin{array}{c}26.3 \\
(12.7)\end{array}$ & $\begin{array}{l}11.6 \\
(4.1)\end{array}$ \\
\hline $10^{\circ}-40 \mathrm{~kg}$-up & $\begin{array}{c}49.3 \\
(18.2)\end{array}$ & $\begin{array}{l}229.3 \\
(24.7)\end{array}$ & $\begin{array}{l}665.3 \\
(79.6)\end{array}$ & $\begin{array}{c}45.8 \\
(22.1)\end{array}$ & $\begin{array}{l}45.3 \\
(9.6)\end{array}$ & $\begin{array}{l}18.4 \\
(5.3)\end{array}$ & $\begin{array}{c}61.9 \\
(10.5)\end{array}$ & $\begin{array}{l}205.2 \\
(37.4)\end{array}$ & $\begin{array}{l}652.7 \\
(69.9)\end{array}$ & $\begin{array}{c}60.9 \\
(22.5)\end{array}$ & $\begin{array}{c}23.1 \\
(11.9)\end{array}$ & $\begin{array}{c}9.8 \\
(5.2)\end{array}$ \\
\hline $10^{\circ}-20 \mathrm{~kg}$-down & $\begin{array}{l}61.8 \\
(9.8)\end{array}$ & $\begin{array}{l}174.1 \\
(45.4)\end{array}$ & $\begin{array}{r}709.1 \\
(54.9)\end{array}$ & $\begin{array}{c}63 \\
(16)\end{array}$ & $\begin{array}{c}46.3 \\
(12.3)\end{array}$ & $\begin{array}{l}11.8 \\
(4.5)\end{array}$ & $\begin{array}{c}71 \\
(22.5)\end{array}$ & $\begin{array}{c}199.4 \\
(38)\end{array}$ & $\begin{array}{l}686.8 \\
(56.9)\end{array}$ & $\begin{array}{c}47.2 \\
(21.3)\end{array}$ & $\begin{array}{c}41 \\
(17.9)\end{array}$ & $\begin{array}{l}15.6 \\
(7.6)\end{array}$ \\
\hline $10^{\circ}-30 \mathrm{~kg}$-down & $\begin{array}{c}63.6 \\
(18.6)\end{array}$ & $\begin{array}{c}163.4 \\
(35)\end{array}$ & $\begin{array}{r}700.3 \\
(53.8) \\
\end{array}$ & $\begin{array}{l}67.4 \\
(9.2)\end{array}$ & $\begin{array}{c}49.3 \\
(21.5)\end{array}$ & $\begin{array}{c}9.8 \\
(4.4)\end{array}$ & $\begin{array}{c}65.3 \\
(23.6)\end{array}$ & $\begin{array}{l}204.6 \\
(35.6)\end{array}$ & $\begin{array}{c}687.6 \\
(64)\end{array}$ & $\begin{array}{c}43.3 \\
(18.9)\end{array}$ & $\begin{array}{c}49.1 \\
(13.2)\end{array}$ & $\begin{array}{l}16.3 \\
(5.3)\end{array}$ \\
\hline $10^{\circ}-40 \mathrm{~kg}$-down & $\begin{array}{c}72.8 \\
(25.9)\end{array}$ & $\begin{array}{l}181.1 \\
(52.5)\end{array}$ & $\begin{array}{l}692.6 \\
(54.7)\end{array}$ & $\begin{array}{c}58.8 \\
(11.4)\end{array}$ & $\begin{array}{c}42.7 \\
(12.6)\end{array}$ & $\begin{array}{c}11.4 \\
(3)\end{array}$ & $\begin{array}{c}62.2 \\
(26.7)\end{array}$ & $\begin{array}{l}206.1 \\
(42.7)\end{array}$ & $\begin{array}{l}689.4 \\
(68.8)\end{array}$ & $\begin{array}{c}40.3 \\
(25.4)\end{array}$ & $\begin{array}{c}45.4 \\
(15.9)\end{array}$ & $\begin{array}{c}16 \\
(9.9)\end{array}$ \\
\hline
\end{tabular}

*Fx,Fy, Fz are in N, and Mx, My, Mz are in $\mathrm{Nm}$. 


\subsubsection{Major joint angle data}

The raw marker data was processed to obtain joint kinematic trajectory for the various joint using the Vicon Nexus software (see Section 4.6.1). The peak joint angle data for major body joints in flexion extension plane are shown in Table 6.3. Raw joint angle data are shown in Appendix G.

\subsection{Model 1: Cart Pushing along a Flat Walkway}

Raw data for the low back and shoulder joint loading across all experimental situations for two sessions are shown in Appendix H.

\subsubsection{Low back joint loading at L5S1}

Table 6.4 shows the mean $\pm \mathrm{SD}$ of the peak compression and shear forces acting at the L5S1 joint during cart pushing tasks performed under three weight conditions. The amount of the weight transferred significantly affected the peak compression, lateral and anterior-posterior shear forces acting at the L5S1 joint (all p < 0.001). The magnitudes of peak forces increased with the increase in the load weight. The magnitude of peak lateral shear force at L5S1 joint was very small compared to the reaction forces in the other two directions. Results of the Allpairwise comparison test (Tukey HSD analysis) showed that the mean of peak lateral shear forces at $20 \mathrm{~kg}$ weight condition was different from both $30 \mathrm{~kg}$ and $40 \mathrm{~kg}$ weight conditions (Table 6.4).

The compression force at the L5S1 joint was higher than the corresponding shear forces. Results of the All-pairwise comparison test showed that the mean of peak compression forces at L5S1 joint were different from one another with respect to three weight conditions (Table 6.4). The shear force at L5S1 joint in the anterior-posterior direction was higher than the respective lateral shear force, but lower than the compression force. Results of the All-pairwise comparison test indicated that the mean of peak shear forces in the anterior-posterior direction at $40 \mathrm{~kg}$ weight condition was different from $20 \mathrm{~kg}$ and $30 \mathrm{~kg}$ weight conditions (Table 6.4). 
Table 6.3: The minimum, maximum and mean of peak joint angles (in degrees) for trunk, right upper and lower limbs across all experimental conditions

\begin{tabular}{|c|c|c|c|c|c|c|c|c|c|c|c|c|c|c|c|c|c|c|}
\hline \multirow{2}{*}{$\begin{array}{l}\text { Experimental } \\
\text { Conditions }\end{array}$} & \multicolumn{3}{|c|}{$\begin{array}{c}\text { Trunk } \\
\text { Flexion/extension }\end{array}$} & \multicolumn{3}{|c|}{$\begin{array}{l}\text { Right - Shoulder } \\
\text { Flexion/extension }\end{array}$} & \multicolumn{3}{|c|}{$\begin{array}{c}\text { Right }- \text { Elbow } \\
\text { Flexion/extension }\end{array}$} & \multicolumn{3}{|c|}{$\begin{array}{c}\text { Right }- \text { Hip } \\
\text { Flexion/extension }\end{array}$} & \multicolumn{3}{|c|}{$\begin{array}{c}\text { Right }- \text { Knee } \\
\text { Flexion/extension }\end{array}$} & \multicolumn{3}{|c|}{$\begin{array}{c}\text { Right }- \text { Ankle } \\
\text { Flexion/extension }\end{array}$} \\
\hline & Min. & Max. & Mean & Min. & Max. & Mean & Min. & Max. & Mean & Min. & Max. & Mean & Min. & Max. & Mean & Min. & Max. & Mean \\
\hline $0^{\circ}-20 \mathrm{~kg}$ & -8.3 & 17.7 & 4.3 & -18.5 & 32.9 & 13 & 30.7 & 99.1 & 67.3 & -35 & 29.3 & 16.3 & 39.2 & 63.2 & 52.8 & -15.9 & 20 & 7.6 \\
\hline $0^{\circ}-30 \mathrm{~kg}$ & -7.3 & 18.1 & 6.5 & -20.5 & 32.2 & 12 & 37.1 & 116.9 & 69.6 & 11.1 & 40.6 & 23.5 & 47.1 & 65.4 & 56.2 & -17.2 & 20.4 & 12.8 \\
\hline $0^{\circ}-40 \mathrm{~kg}$ & -10.4 & 19.7 & 5.6 & -23.2 & 35.6 & 7.6 & 35.7 & 114.4 & 65.8 & 10.9 & 40.4 & 24.1 & 41.7 & 65.3 & 56.2 & -18.6 & 20.6 & 10.6 \\
\hline $5^{\circ}-20 \mathrm{~kg}$-up & -4.5 & 30.0 & 9.4 & -7.2 & 45.3 & 25.2 & 39 & 119.2 & 68.1 & 24.2 & 49.4 & 33.3 & 49.9 & 73.6 & 58.2 & -24.5 & 24.7 & 13.5 \\
\hline $5^{\circ}-30 \mathrm{~kg}$-up & -6.8 & 25.1 & 14.3 & 22 & 65.5 & 40 & 39 & 73.2 & 62.6 & 27.1 & 51.7 & 36.8 & 50.9 & 71.4 & 57.4 & -21.1 & 26.8 & 19.4 \\
\hline $5^{\circ}-40 \mathrm{~kg}$-up & -5.8 & 40.4 & 13.9 & -5.5 & 72.5 & 36.1 & 42.3 & 82.3 & 61 & 29.2 & 53.9 & 40.2 & 41.3 & 70.5 & 58.7 & -25.1 & 29.5 & 21.5 \\
\hline $5^{\circ}-20 \mathrm{~kg}$-down & -7.0 & 27.4 & 7.2 & 10.7 & 41 & 21 & 44.1 & 83.5 & 58.7 & 11.1 & 52.7 & 20.2 & 19 & 78.5 & 57.1 & -26.9 & 28.5 & 12.7 \\
\hline $5^{\circ}-30 \mathrm{~kg}$-down & 3.3 & 38.8 & 14.3 & -172 & 37 & 2.6 & 39.1 & 97.8 & 58.4 & -4 & 54.7 & 21.1 & 52.2 & 79.3 & 65.9 & -27.4 & 30.2 & 17.3 \\
\hline $5^{\circ}-40 \mathrm{~kg}$-down & 5.3 & 55.5 & 14.8 & -9.5 & 51.6 & 18 & 37.1 & 89.6 & 55.9 & -29.7 & 54.3 & 15.2 & 55.7 & 75.2 & 66.7 & -25.1 & 29.4 & 14.1 \\
\hline $10^{\circ}$-20kg-up & 6.3 & 29.8 & 16.5 & 16.5 & 62.9 & 40.3 & 55 & 85.9 & 68.7 & 35.4 & 64.8 & 44.1 & 45.1 & 74.1 & 58.9 & -27.9 & 35.3 & 24.8 \\
\hline $10^{\circ}-30 \mathrm{~kg}$-up & -8.3 & 29.7 & 16.9 & -21.2 & 75.9 & 37.2 & 42.7 & 100 & 72.9 & 38.5 & 68.8 & 49.5 & 50.1 & 77.4 & 60.6 & -48.5 & 36.8 & 20.3 \\
\hline $10^{\circ}-40 \mathrm{~kg}$-up & -5.2 & 28.4 & 15.4 & -11.9 & 85.5 & 30.4 & 43.9 & 131.1 & 87 & 45.9 & 72.2 & 54.8 & 55.1 & 73.9 & 63.3 & -47.3 & 43 & 28.2 \\
\hline $10^{\circ}$-20kg-down & 3.9 & 36.7 & 16.8 & -5.4 & 41.2 & 20.4 & 38.2 & 108.4 & 62.3 & -14.8 & 34.2 & 18.9 & 57.9 & 79.9 & 69.7 & -35.1 & 26.5 & 11.2 \\
\hline $10^{\circ}-30 \mathrm{~kg}$-down & 8.9 & 32.8 & 17.6 & 8.1 & 58.1 & 27.5 & 35.9 & 70.9 & 54.3 & 6.6 & 38.8 & 24.1 & 66.2 & 87.4 & 71.9 & -38 & 27.3 & 12.7 \\
\hline $10^{\circ}$-40kg-down & 4.5 & 36.9 & 16.4 & 3.4 & 66.7 & 25.2 & 41 & 84.2 & 54.3 & 15.2 & 41.3 & 26 & 60.4 & 80.6 & 71.5 & -43.1 & 27.1 & 9.9 \\
\hline
\end{tabular}


Table 6.4: Mean (SD) of the peak forces acting at the L5S1 joint during pushing task performed on a flat walkway

\begin{tabular}{|c|c|c|c|c|}
\hline & $20 \mathrm{~kg}$ & $30 \mathrm{~kg}$ & $40 \mathrm{~kg}$ & p-value \\
\hline L5S1-ML & $43.6(23.2)^{\mathrm{a}}$ & $63.0(32.7)^{\mathrm{b}}$ & $77.9(38.9)^{\mathrm{b}}$ & $<0.001^{*}$ \\
\hline L5S1-PD & $863.0(95.1)^{\mathrm{a}}$ & $1066.2(172.8)^{\mathrm{b}}$ & $1432.3(252.6)^{\mathrm{c}}$ & $<0.001^{*}$ \\
\hline L5S1-AP & $170.3(58.3)^{\mathrm{a}}$ & $222.4(87.5)^{\mathrm{a}}$ & $331.5(104.4)^{\mathrm{b}}$ & $<0.001^{*}$ \\
\hline
\end{tabular}

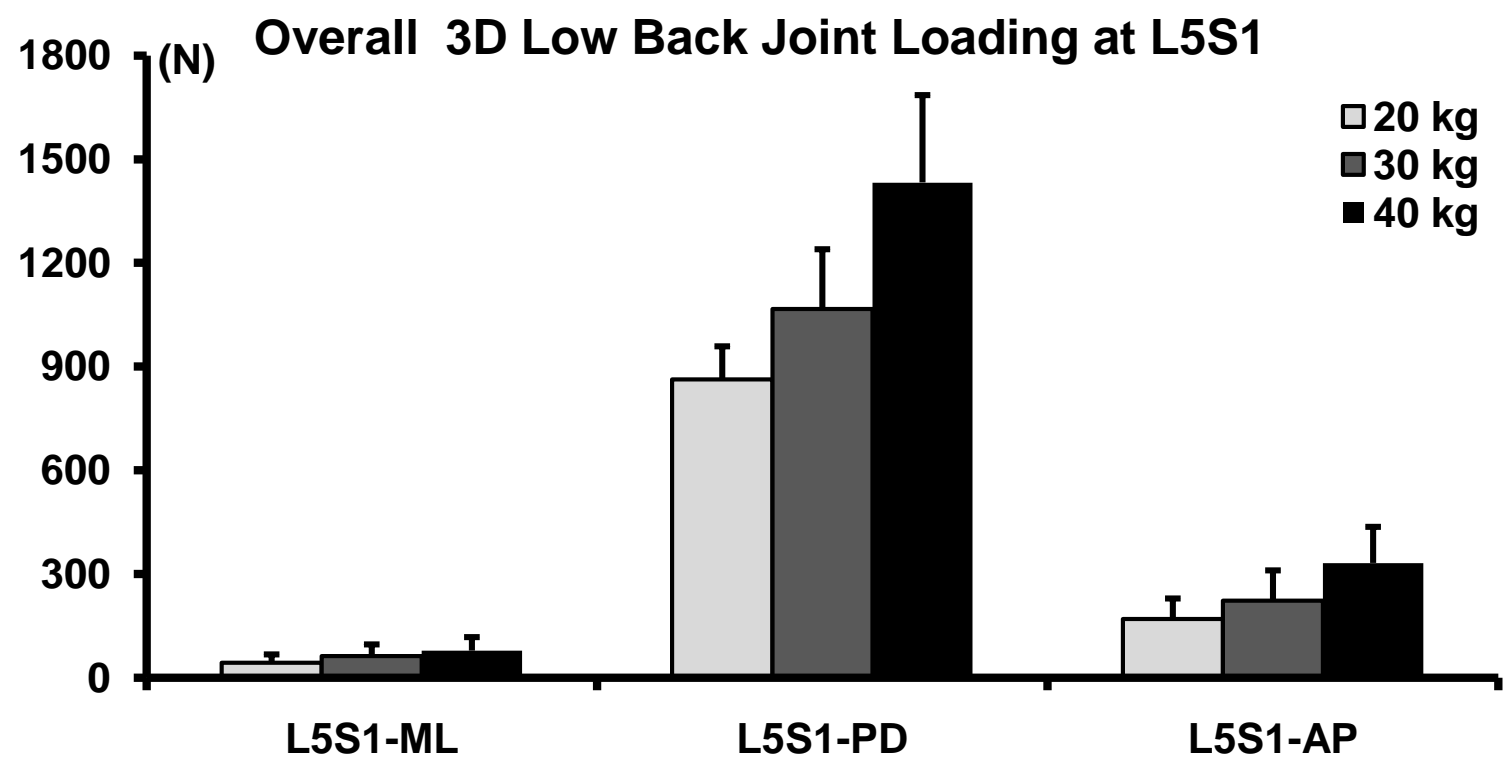

Figure 6.1: Peak reaction forces acting at the L5S1 joint in three anatomical directions

\subsubsection{Shoulder joint loading} as a function of load weight

\subsubsection{Sternoclavicluar (SC) joint loading}

The peak forces (mean $\pm \mathrm{SD}$ ) acting at the sternoclavicular (SC) joint in three anatomical directions during the cart pushing task performed under three weight conditions are shown in Table 6.5. The amount of the load weight had a significant effect on the peak compression, lateral and anterior-posterior shear forces at the SC joint (all $\mathrm{p}<0.001$ ). The magnitudes of peak forces increased with the increase in the load weight.

Table 6.5: Mean (SD) of the peak forces acting at the sternoclavicular (SC) joint during pushing task performed on a flat walkway

\begin{tabular}{|c|c|c|c|c|}
\hline & $20 \mathrm{~kg}$ & $30 \mathrm{~kg}$ & $40 \mathrm{~kg}$ & $\mathrm{p}$-value \\
\hline RSC - ML & $48.2(19.0)^{\mathrm{a}}$ & $63.5(24.8)^{\mathrm{b}}$ & $76.0(24.2)^{\mathrm{c}}$ & $<0.001^{*}$ \\
\hline RSC - IS & $52.0(16.7)^{\mathrm{a}}$ & $74.2(17.0)^{\mathrm{b}}$ & $111.5(30.7)^{\mathrm{c}}$ & $<0.001^{*}$ \\
\hline RSC - AP & $24.2(8.5)^{\mathrm{a}}$ & $31.9(13.7)^{\mathrm{b}}$ & $41.9(13.4)^{\mathrm{c}}$ & $<0.001^{*}$ \\
\hline
\end{tabular}


The magnitude of the peak lateral shear force at the SC joint was higher than the respective anterior-posterior shear force, but lower than the compression force. Results of the All-pairwise comparison test (Tukey HSD analysis) showed that the mean of peak lateral shear forces at the SC joint were different from one another with respect to three weight conditions (Table 6.5). For the compression force in the inferior-superior direction and shear force in the anterior-posterior direction mean of forces at the SC joint were different from one another with respect to three weight conditions (Table 6.5).

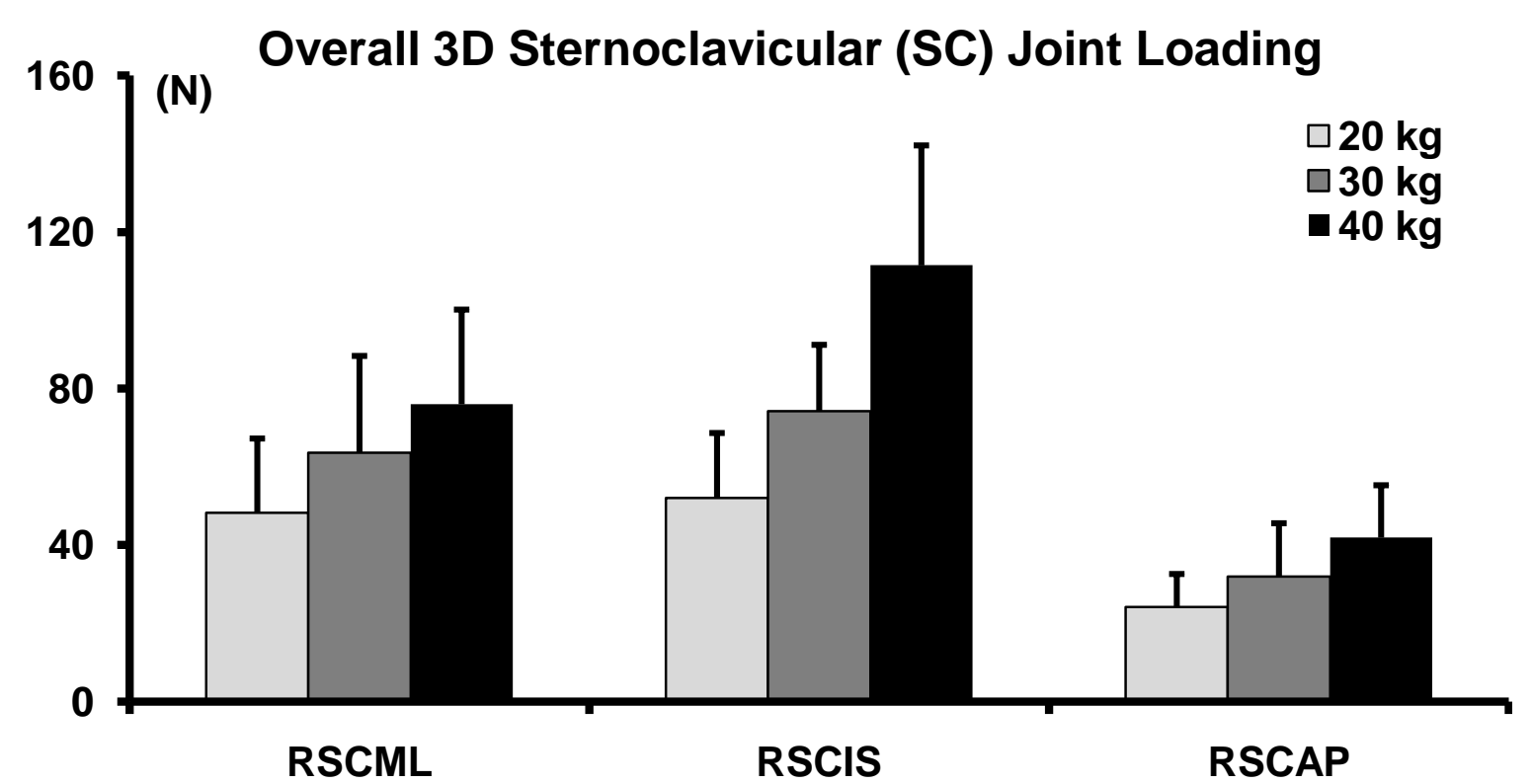

Figure 6.2: Peak reaction forces acting at the sternoclavicular (SC) joint in three anatomical directions as a function of load weight

\subsubsection{Acromioclavicluar (AC) joint loading}

The peak forces (mean $\pm \mathrm{SD}$ ) acting at the acromioclavicular (AC) joint during cart pushing performed under three weight conditions are shown in Table 6.6. The load weight significantly affected the peak compression, lateral and anterior-posterior shear forces at the AC joint (all p < 0.001). The magnitudes of peak forces increased with the increase in the load weight from $20 \mathrm{~kg}$ to $30 \mathrm{~kg}$ to $40 \mathrm{~kg}$.

Table 6.6: Mean (SD) of the peak forces acting at the acromioclavicular (AC) joint during pushing task performed on a flat walkway

\begin{tabular}{|c|c|c|c|c|}
\hline & $20 \mathrm{~kg}$ & $30 \mathrm{~kg}$ & $40 \mathrm{~kg}$ & $\mathrm{p}$-value \\
\hline RAC - ML & $204.0(36.7)^{\mathrm{a}}$ & $308.2(63.0)^{\mathrm{b}}$ & $404.3(83.8)^{\mathrm{c}}$ & $<0.001^{*}$ \\
\hline RAC - IS & $269.4(62.6)^{\mathrm{a}}$ & $381.8(88.6)^{\mathrm{b}}$ & $543.7(112.1)^{\mathrm{c}}$ & $<0.001^{*}$ \\
\hline RAC - AP & $168.0(54.4)^{\mathrm{a}}$ & $225.7(62.2)^{\mathrm{b}}$ & $325.4(92.9)^{\mathrm{c}}$ & $<0.001^{*}$ \\
\hline
\end{tabular}


The lateral shear force at the AC joint was higher than the respective anterior-posterior shear force, but lower than the compression force in the inferior-superior direction. Results of the All-pairwise comparison test (Tukey HSD) showed that the mean of peak lateral shear forces were different from one another with respect to three weight conditions (Table 6.6). The same trend was found for the peak forces acting at the $\mathrm{AC}$ joint in the other two directions.

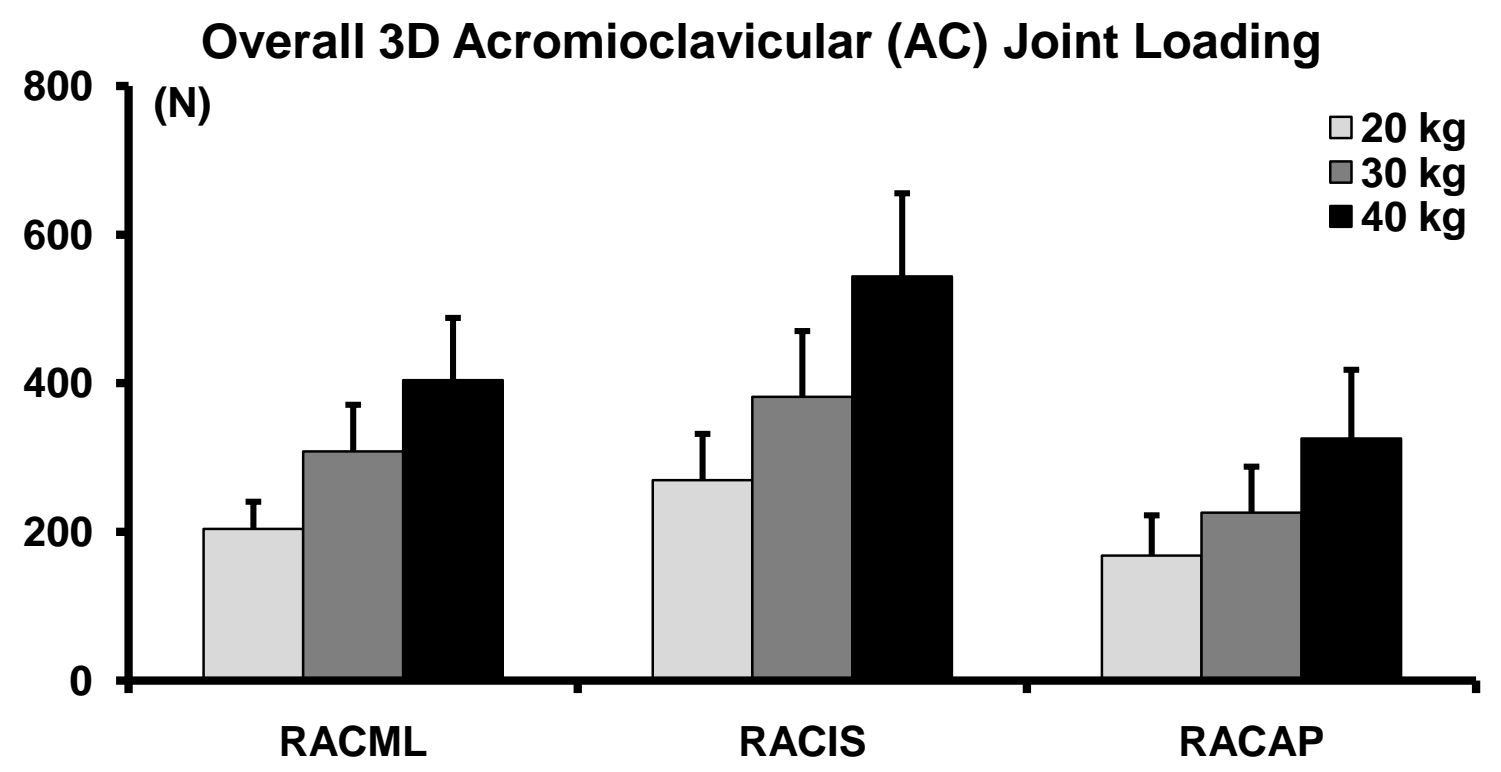

Figure 6.3: Peak reaction forces acting at the acromioclavicular (AC) joint in the three anatomical direction as a function of load weight

\subsubsection{Glenohumeral (GH) joint loading}

During the pushing task on a flat walkway, the amount of the load weight transferred also significantly affected the peak compression, lateral and anterior-posterior shear forces acting at the GH joint (all p < 0.001) (Table 6.7). A general trend was found that the magnitudes of peak forces increased with the increase in the load weight.

Table 6.7: Mean (SD) of the peak forces acting at the glenohumeral (GH) joint during pushing task performed on a flat walkway

\begin{tabular}{|c|c|c|c|c|}
\hline & $20 \mathrm{~kg}$ & $30 \mathrm{~kg}$ & $40 \mathrm{~kg}$ & $\mathrm{p}$-value \\
\hline RGH - DIS & $344.1(83.0)^{\mathrm{a}}$ & $479.5(88.9)^{\mathrm{b}}$ & $681.0(121.3)^{\mathrm{c}}$ & $<0.001^{*}$ \\
\hline RGH - IS & $192.3(83.6)^{\mathrm{a}}$ & $294.6(136.1)^{\mathrm{a}}$ & $454.6(150.9)^{\mathrm{b}}$ & $<0.001^{*}$ \\
\hline RGH - AP & $166.2(43.2)^{\mathrm{a}}$ & $249.3(88.4)^{\mathrm{b}}$ & $316.2(92.7)^{\mathrm{c}}$ & $<0.001^{*}$ \\
\hline
\end{tabular}




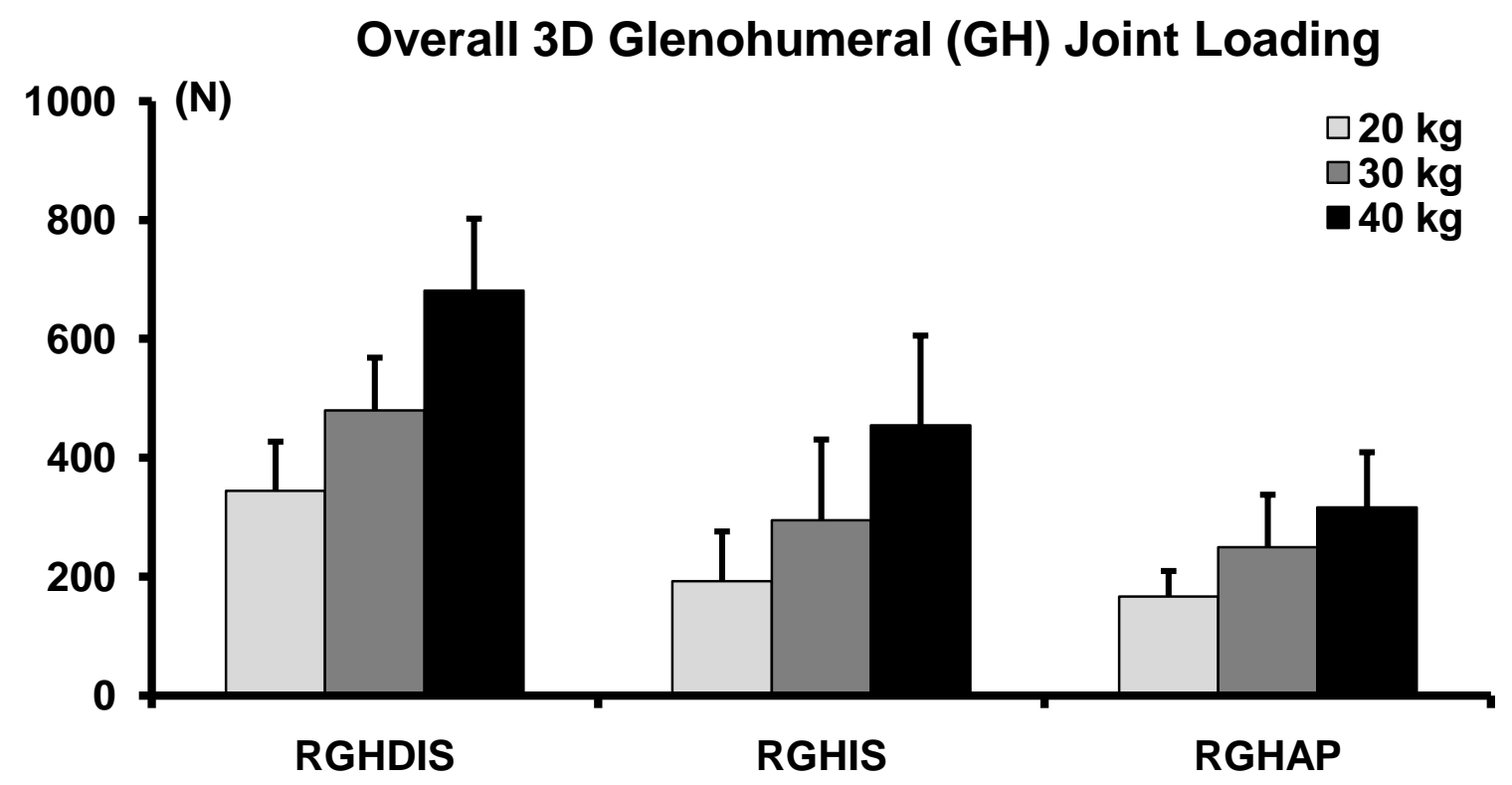

Figure 6.4: Peak reaction forces acting at the glenohumeral $(\mathrm{GH})$ joint in three anatomical directions as a function of load weight

For the GH joint, among the three anatomical directions, the highest forces were exerted in the distraction direction followed by the inferior-superior and anterior-posterior directions. Results of the All-pairwise comparison test (Tukey HSD analysis) showed that the mean of peak shear forces in the distraction as well as the anterior-posterior direction was different from one another with respect to three weight conditions (Table 6.7). For the compression force in the inferior-superior direction, the mean of peak forces acting at the $\mathrm{GH}$ joint at $40 \mathrm{~kg}$ weight condition was different from the $20 \mathrm{~kg}$ and $30 \mathrm{~kg}$ weight conditions (Table 6.7).

\subsection{Model 2: Cart Pushing and Pulling along Ramps}

\subsubsection{Low back joint loading at L5S1}

\subsubsection{Main effects}

A general trend in the data indicates that the peak compression and shear forces acting at the L5S1 joint increased with the increase in the load weights and the ramp heights (Figure 6.5). Among the two directions of force application, higher joint loading was observed during uphill pushing than downhill pulling. Statistically, the main effect of load weight, walkway gradient, and direction of force exertion on the peak L5S1 compression and shear forces in the anteriorposterior (AP) direction was significant (all $\mathrm{p} \leq 0.01$ ). The shear force in the lateral (ML) direction at the L5S1 joint was not affected by the walkway gradient $(\mathrm{p}=0.52)$, but was 
significantly affected by load weight and direction of force application $(\mathrm{p}<0.01)$. The magnitude of the lateral shear force at the L5S1 joint was very small compared to the reaction forces in the other two directions ( $<150 \mathrm{~N}$ in all situations).
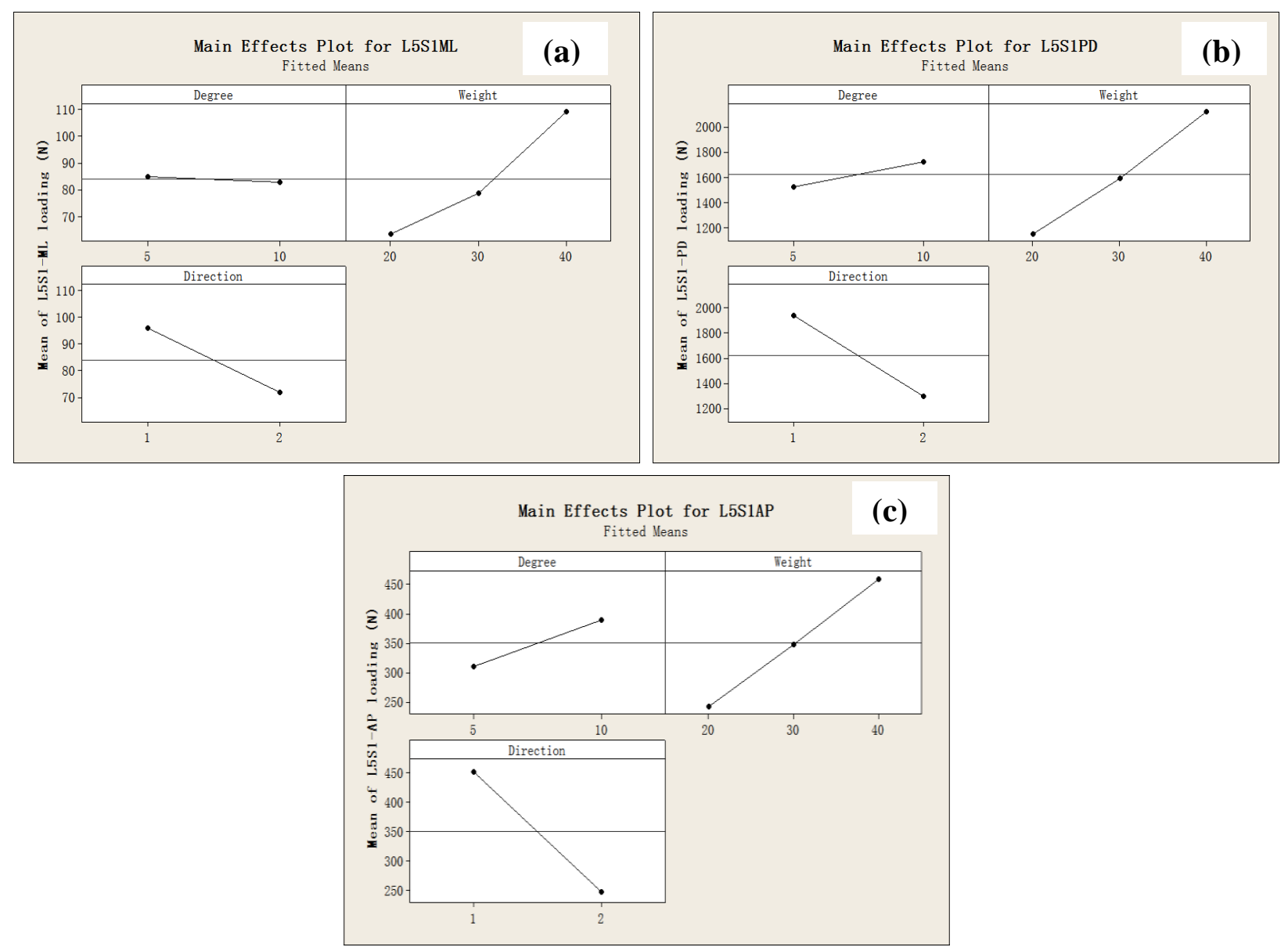

Figure 6.5: Main effect plots for the loading of the L5S1 joint in three directions ((a) L5S1-ML; (b) L5S1-PD; (c) L5S1-AP) by the walkway gradient, load weight, and direction of force application

\subsubsection{Two-way interaction effects}

The interaction effect between the walkway gradient and load weight was significant for the peak shear force in the AP direction at the L5S1 joint $(\mathrm{p}=0.04)$. This interaction effect was ordinal, suggesting that at the $40 \mathrm{~kg}$ weight condition, increase in the walkway gradient caused a relatively higher increase in the peak shear force in the AP direction than at 20 and $30 \mathrm{~kg}$ weight conditions (Figure 6.6 (c)). The interaction effect between the walkway gradient and direction of force exertion was significant for the peak compression and shear forces acting at the L5S1 joint (all $\mathrm{p} \leq 0.01$ ). A 
disordinal interaction effect was observed, meaning that with the increase in the walkway gradient from $5^{\circ}$ to $10^{\circ}$, compression and shear forces acting at the L5S1 joint increased during uphill pushing. During downhill pulling, these forces decreased with the increase in the walkway gradient from $5^{\circ}$ to $10^{\circ}$. An ordinal interaction effect was also observed between load weight and direction of force application for the loading of the L5S1 joint in the three directions (all $\mathrm{p} \leq 0.01$ ). During uphill pushing, an increase in the weight of the cart caused a relatively higher increase in the 3D loading of the L5S1 joint than downhill pulling.
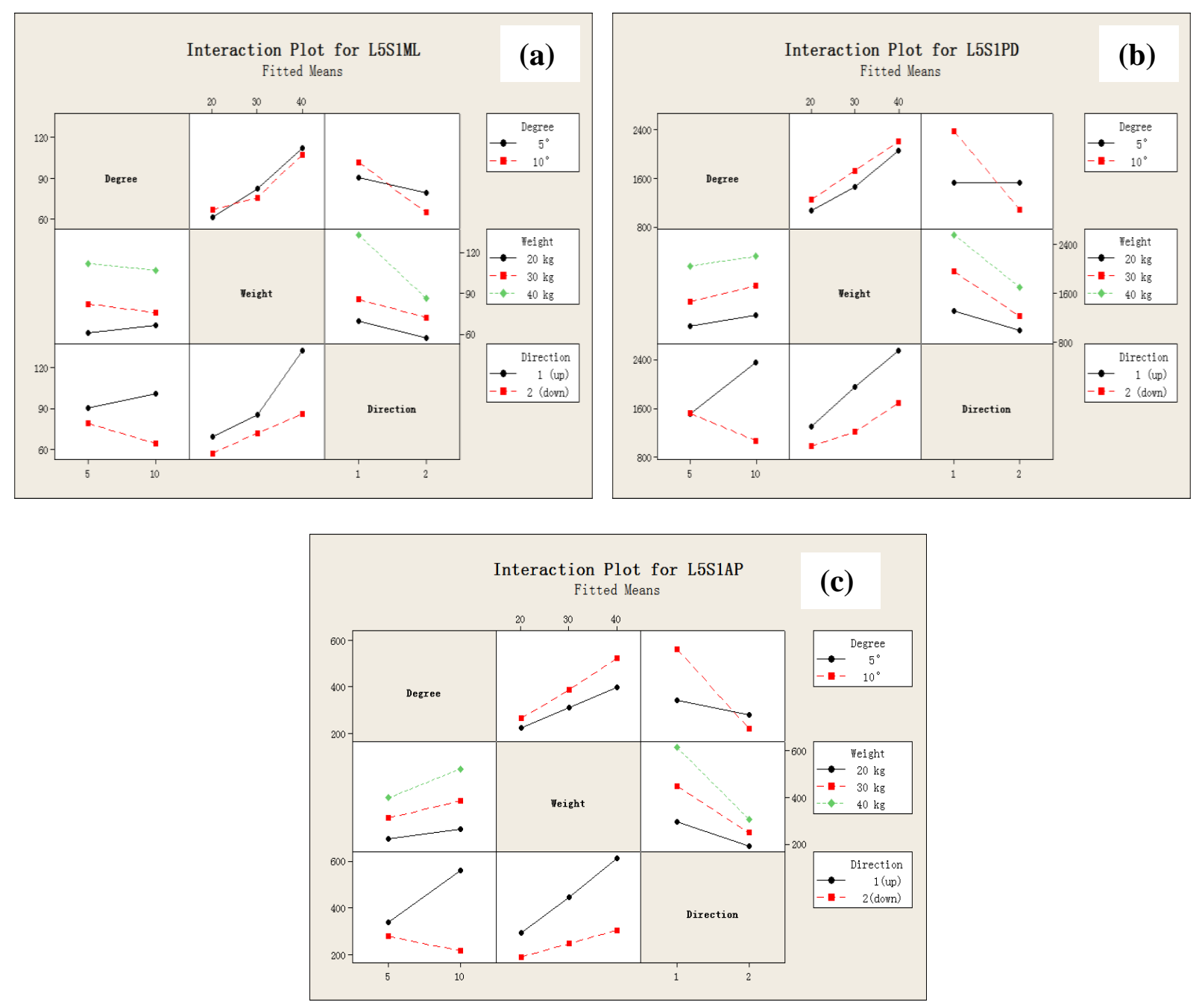

Figure 6.6: Two-way interaction plots for the loading of the L5S1 joint in three directions: (a) L5S1-ML; (b) L5S1-PD; (c) L5S1-AP 


\subsubsection{Three-way interaction effect and Tukey HSD analysis}

The dataset was unstacked by the gradient $\left(5^{\circ}\right.$ and $\left.10^{\circ}\right)$ to facilitate the interpretation of the three-way interaction effect (Figure 6.7). Three-way interaction effect between walkway gradient, load weight, and direction of force application was significant for the peak compression and anterior-posterior shear forces acting at the L5S1 joint (both $\mathrm{p}<0.01$ ) (Figures $6.8-6.10$ ).

At $5^{\circ}$ gradient increase in the load weight increased the peak compression, lateral and anterior-posterior shear forces during uphill pushing as well as downhill pulling. Results of the All-pairwise comparison test showed that the mean of peak compression, lateral and anteriorposterior shear forces at $40 \mathrm{~kg}$ weight condition was different from $20 \mathrm{~kg}$ weight condition during uphill pushing and downhill pulling. During downhill pulling, the mean of peak compression forces at $40 \mathrm{~kg}$ weight was also different from $30 \mathrm{~kg}$ weight condition. A comparison between the directions of force application indicates that the mean of peak lateral forces during uphill pushing at $40 \mathrm{~kg}$ weight was higher than downhill pulling.

On the $10^{\circ}$ ramp, during uphill pushing, the mean of peak compression and anteriorposterior shear forces at 20,30, and $40 \mathrm{~kg}$ weight conditions were different from one another, while the mean of peak lateral forces at $40 \mathrm{~kg}$ weight was different from $20 \mathrm{~kg}$ and $30 \mathrm{~kg}$ weight. No significant difference was found between the three weight conditions during downhill pulling. A between direction of force application comparison indicates that the mean of peak compression, lateral, anterior-posterior shear forces during uphill pushing at $30 \mathrm{~kg}$ and $40 \mathrm{~kg}$ weight conditions was higher than downhill pulling. Additionally, the mean of peak anteriorposterior shear forces during uphill pushing at $20 \mathrm{~kg}$ weight was higher than downhill pulling.

A between gradient comparison indicates that the mean of peak compression and anterior-posterior shear forces during uphill pushing under $30 \mathrm{~kg}$ and $40 \mathrm{~kg}$ load weight conditions were higher along the $10^{\circ}$ ramp than the $5^{\circ}$ ramp. 

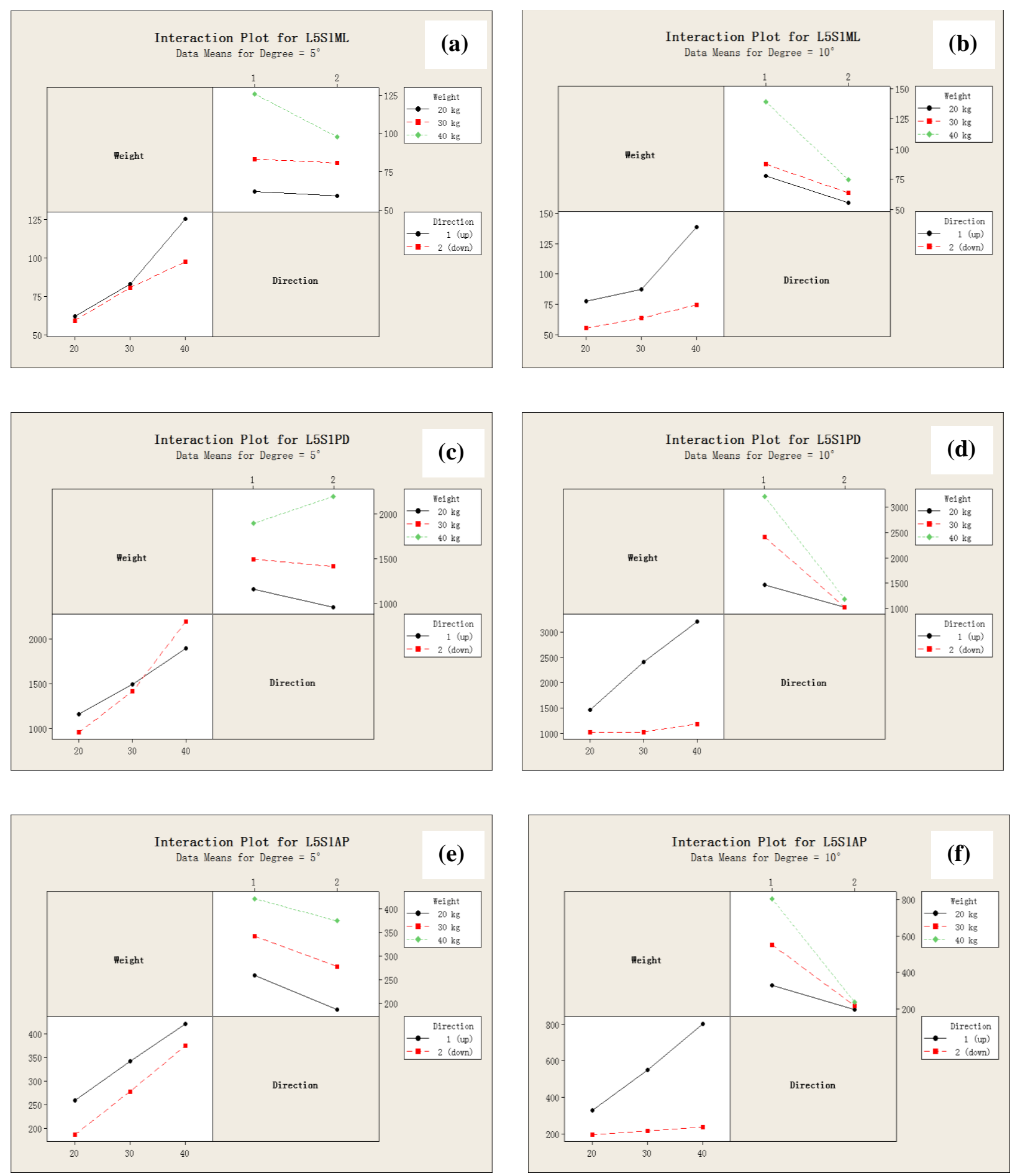

Figure 6.7: Three-way interaction plots for the loading of the L5S1 joint unstacked by the walkway gradient (degree) factor 


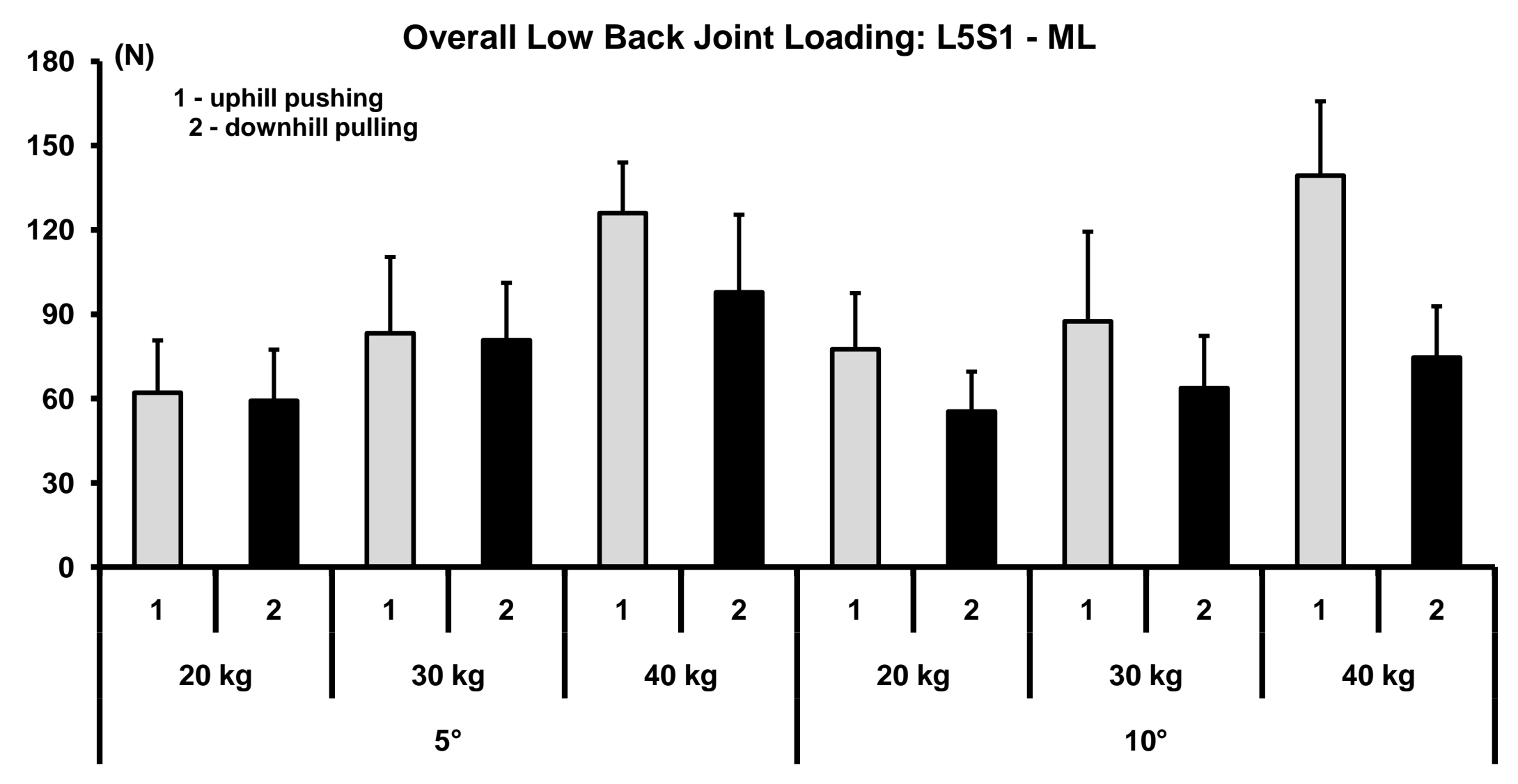

\begin{tabular}{|c|c|c|c|c|c|c|c|c|c|c|c|c|c|c|c|c|c|c|}
\hline \multicolumn{6}{|c|}{$5^{\circ}$} & \multicolumn{6}{|c|}{$10^{\circ}$} & \multirow{2}{*}{\multicolumn{7}{|c|}{ p-value }} \\
\hline \multicolumn{2}{|c|}{$20 \mathrm{~kg}$} & \multicolumn{2}{|c|}{$30 \mathrm{~kg}$} & \multicolumn{2}{|c|}{$40 \mathrm{~kg}$} & \multicolumn{2}{|c|}{$20 \mathrm{~kg}$} & \multicolumn{2}{|c|}{$30 \mathrm{~kg}$} & \multicolumn{2}{|c|}{$40 \mathrm{~kg}$} & & & & & & & \\
\hline 1 & 2 & 1 & 2 & 1 & 2 & 1 & 2 & 1 & 2 & 1 & 2 & $A^{*}$ & B & $\mathrm{C}$ & $A \times B$ & $\mathrm{~A} \times \mathrm{C}$ & $\mathrm{B} \times \mathrm{C}$ & $\mathrm{A} \times \mathrm{B} \times \mathrm{C}$ \\
\hline $\begin{array}{c}62 \\
(19) \\
\end{array}$ & $\begin{array}{c}59 \\
(18) \\
\end{array}$ & $\begin{array}{c}83 \\
(27) \\
\end{array}$ & $\begin{array}{c}81 \\
(20) \\
\end{array}$ & $\begin{array}{l}126 \\
(18) \\
\end{array}$ & $\begin{array}{c}98 \\
(28) \\
\end{array}$ & $\begin{array}{c}78 \\
(20) \\
\end{array}$ & $\begin{array}{c}55 \\
(14) \\
\end{array}$ & $\begin{array}{c}88 \\
(32) \\
\end{array}$ & $\begin{array}{c}64 \\
(19) \\
\end{array}$ & $\begin{array}{l}139 \\
(27) \\
\end{array}$ & $\begin{array}{c}75 \\
(18) \\
\end{array}$ & 0.52 & $<0.01$ & $<0.01$ & 0.18 & $<0.01$ & $<0.01$ & 0.42 \\
\hline
\end{tabular}

Figure 6.8: Peak force acting at the L5S1 joint in the medio-lateral direction during uphill pushing and downhill pulling 


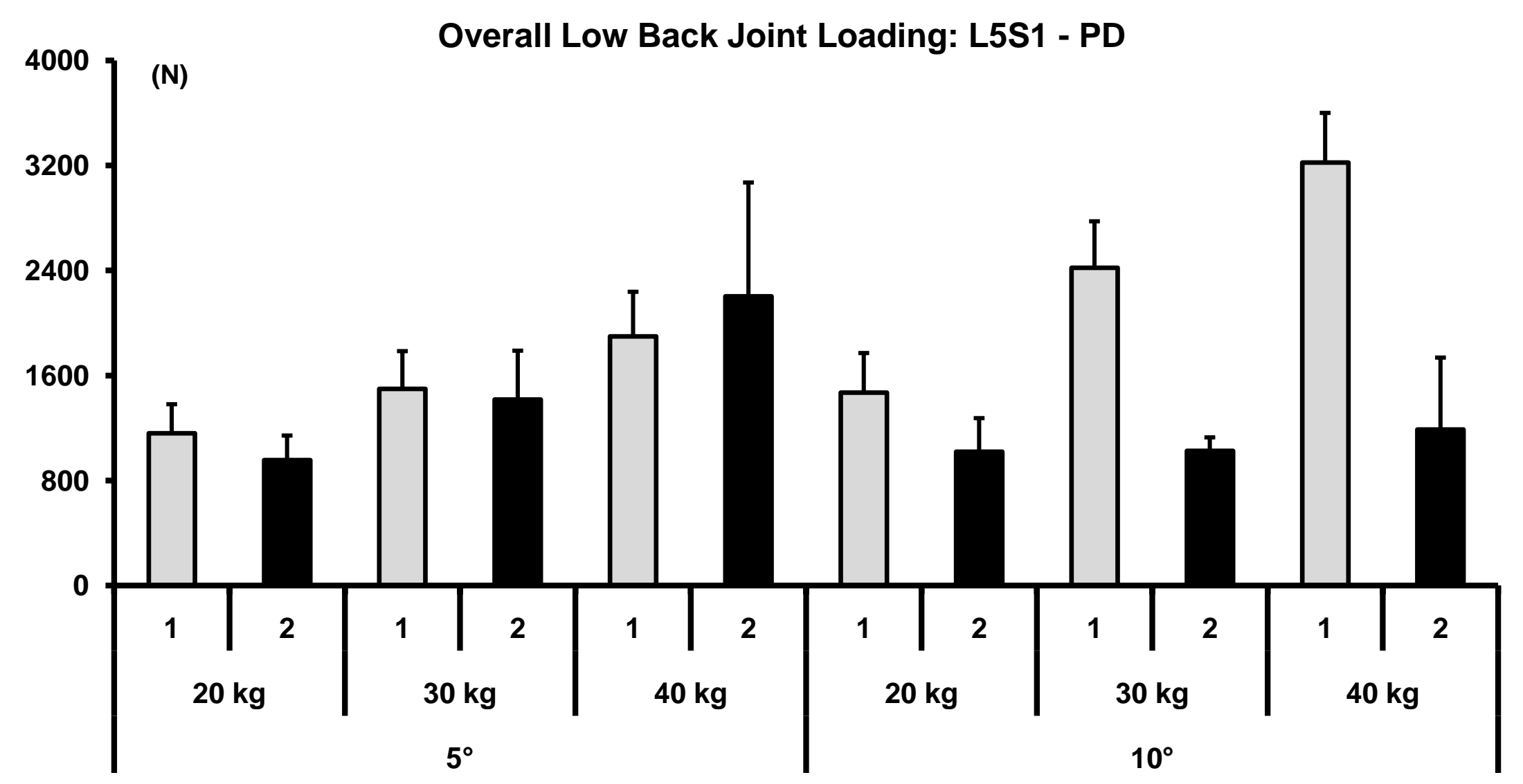

\begin{tabular}{|c|c|c|c|c|c|c|c|c|c|c|c|c|c|c|c|c|c|c|}
\hline \multicolumn{6}{|c|}{$5^{\circ}$} & \multicolumn{6}{|c|}{$10^{\circ}$} & \multirow{2}{*}{\multicolumn{7}{|c|}{ p-value }} \\
\hline \multicolumn{2}{|c|}{$20 \mathrm{~kg}$} & \multicolumn{2}{|c|}{$30 \mathrm{~kg}$} & \multicolumn{2}{|c|}{$40 \mathrm{~kg}$} & \multicolumn{2}{|c|}{$20 \mathrm{~kg}$} & \multicolumn{2}{|c|}{$30 \mathrm{~kg}$} & \multicolumn{2}{|c|}{$40 \mathrm{~kg}$} & & & & & & & \\
\hline 1 & 2 & 1 & 2 & 1 & 2 & 1 & 2 & 1 & 2 & 1 & 2 & $\mathrm{~A}^{*}$ & B & $\mathrm{C}$ & $A \times B$ & $\mathrm{~A} \times \mathrm{C}$ & $\mathrm{B} \times \mathrm{C}$ & $\mathrm{A} \times \mathrm{B} \times \mathrm{C}$ \\
\hline $\begin{array}{l}1160 \\
(221)\end{array}$ & $\begin{array}{c}956 \\
(187)\end{array}$ & $\begin{array}{l}1499 \\
(287)\end{array}$ & $\begin{array}{l}1416 \\
(373)\end{array}$ & $\begin{array}{l}1899 \\
(339)\end{array}$ & $\begin{array}{l}2204 \\
(866)\end{array}$ & $\begin{array}{l}1469 \\
(302)\end{array}$ & $\begin{array}{l}1019 \\
(256)\end{array}$ & $\begin{array}{l}2420 \\
(354)\end{array}$ & $\begin{array}{l}1027 \\
(102)\end{array}$ & $\begin{array}{l}3222 \\
(378)\end{array}$ & $\begin{array}{c}1189 \\
(548)\end{array}$ & 0.01 & $<0.01$ & $<0.01$ & 0.74 & $<0.01$ & 0.01 & $<0.01$ \\
\hline
\end{tabular}

Figure 6.9: Peak force acting at the L5S1 joint in the compression (proximal-distal) direction during uphill pushing and downhill pulling 


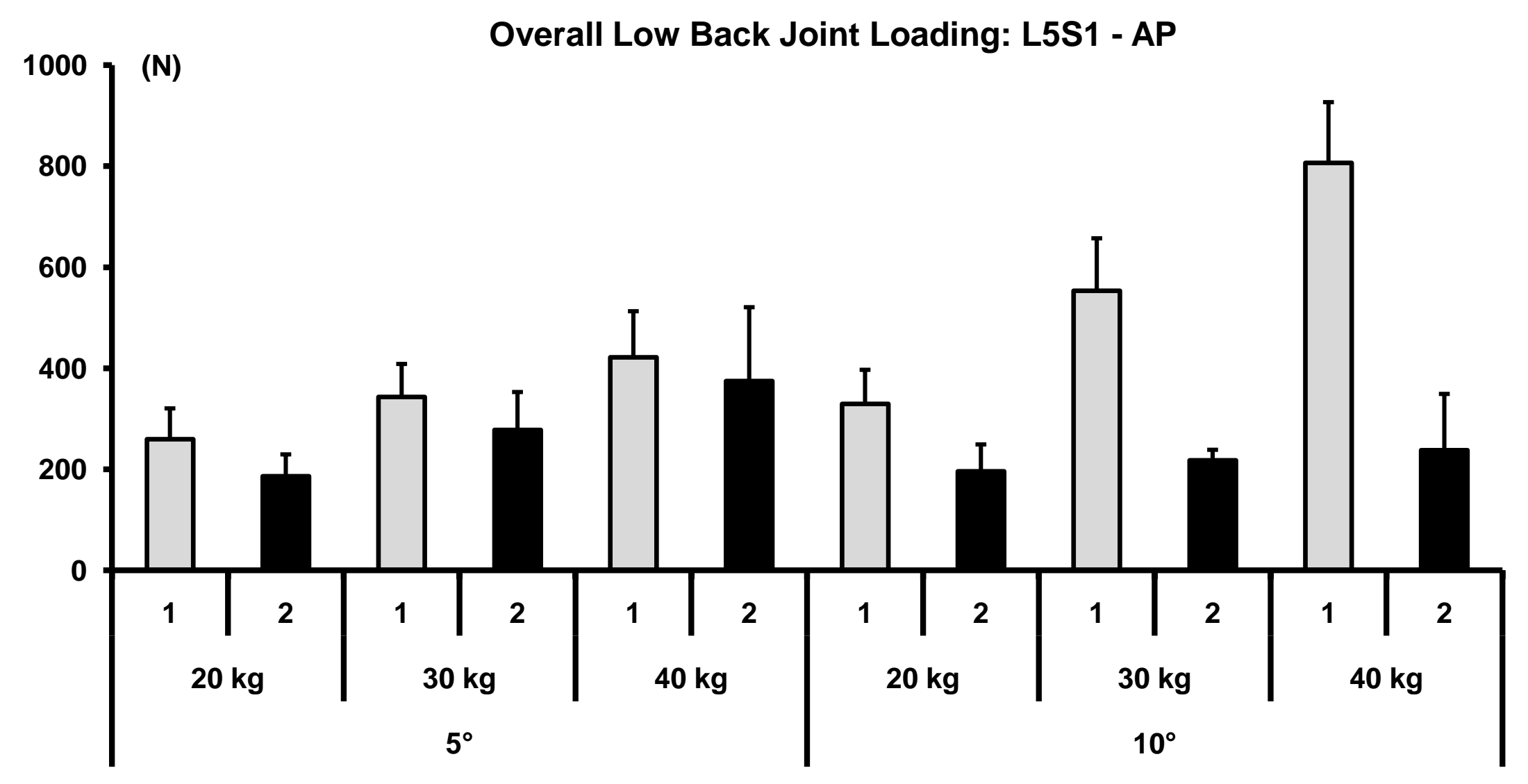

\begin{tabular}{|c|c|c|c|c|c|c|c|c|c|c|c|c|c|c|c|c|c|c|}
\hline \multicolumn{6}{|c|}{$5^{\circ}$} & \multicolumn{6}{|c|}{$10^{\circ}$} & \multirow{2}{*}{\multicolumn{7}{|c|}{ p-value }} \\
\hline \multicolumn{2}{|c|}{$20 \mathrm{~kg}$} & \multicolumn{2}{|c|}{$30 \mathrm{~kg}$} & \multicolumn{2}{|c|}{$40 \mathrm{~kg}$} & \multicolumn{2}{|c|}{$20 \mathrm{~kg}$} & \multicolumn{2}{|c|}{$30 \mathrm{~kg}$} & \multicolumn{2}{|c|}{$40 \mathrm{~kg}$} & & & & & & & \\
\hline 1 & 2 & 1 & 2 & 1 & 2 & 1 & 2 & 1 & 2 & 1 & 2 & $\mathrm{~A}^{*}$ & B & $\mathrm{C}$ & $A \times B$ & $\mathrm{~A} \times \mathrm{C}$ & $\mathrm{B} \times \mathrm{C}$ & $\mathrm{A} \times \mathrm{B} \times \mathrm{C}$ \\
\hline $\begin{array}{l}260 \\
(61)\end{array}$ & $\begin{array}{l}187 \\
(43)\end{array}$ & $\begin{array}{l}343 \\
(66)\end{array}$ & $\begin{array}{l}278 \\
(75)\end{array}$ & $\begin{array}{l}422 \\
(91)\end{array}$ & $\begin{array}{c}375 \\
(146)\end{array}$ & $\begin{array}{l}329 \\
(68)\end{array}$ & $\begin{array}{l}196 \\
(53)\end{array}$ & $\begin{array}{c}553 \\
(104)\end{array}$ & $\begin{array}{l}218 \\
(21)\end{array}$ & $\begin{array}{c}806 \\
(121)\end{array}$ & $\begin{array}{c}238 \\
(112)\end{array}$ & $<0.01$ & $<0.01$ & $<0.01$ & 0.04 & $<0.01$ & $<0.01$ & $<0.01$ \\
\hline
\end{tabular}

Figure 6.10: Peak force acting at the L5S1 joint in the anterior-posterior direction during uphill pushing and downhill pulling 


\subsubsection{Shoulder joint loading at sternoclavicular joint}

\subsubsection{Main effects}

Generally, the peak compression and shear forces acting at the sternoclavicular (SC) joint increased with the load weight, walkway gradient, and direction of force application (Figure 6.11). Statistically, the main effect of load weight, walkway gradient, and direction of force exertion on the peak SC joint compression and shear forces was significant (all p <0.01). The joint loading increased with the increase in the load weight and the walkway gradient. The main effect of direction of force application was also significant, where larger joint forces were observed during uphill pushing than downhill pulling.

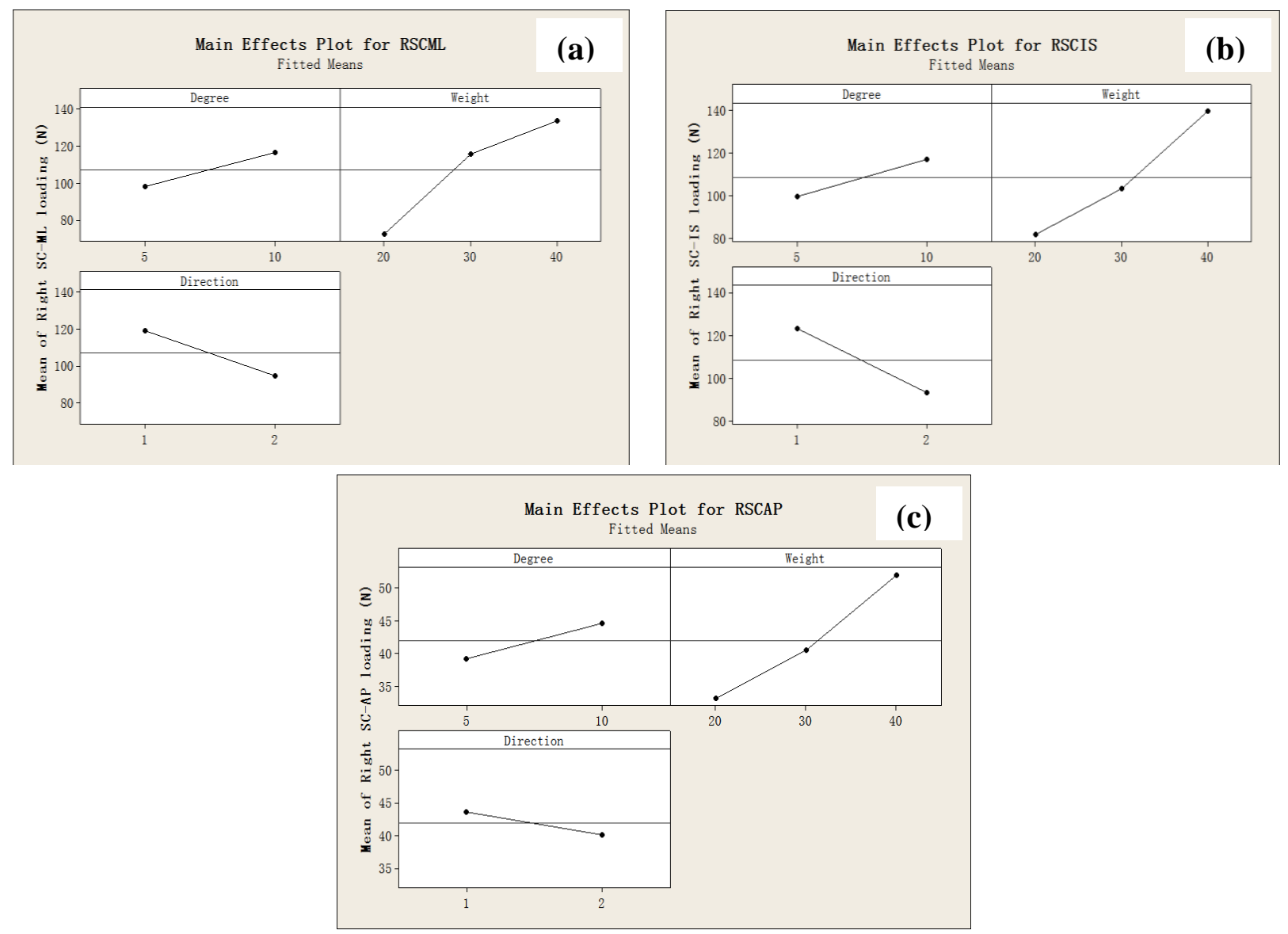

Figure 6.11: Main effect plots for the loading of the sternoclavicular (SC) joint in three directions ((a) RSC-ML; (b) RSC-IS; (c) RSC-AP) by the walkway gradient, load weight, and direction of force application 
Overall, the magnitude of shear force at the SC joint in the anterior-posterior direction was very negligible ( $<65 \mathrm{~N}$ in all situations) compared to the peak forces in the other two directions.

\subsubsection{Two-way interaction effects}

Two-way interaction effects are shown in Figure 6.12. The interaction effect of load weight by walkway gradient was significant for the peak compression and shear forces acting at the SC joint $(\mathrm{p} \leq 0.01)$. The loading at $10^{\circ}$ walkway gradient combined with $40 \mathrm{~kg}$ load weight condition was higher than all the weight conditions at $5^{\circ}$ walkway gradient. The disordinal interaction between walkway gradient and direction of force application was significant for the peak forces acting at the SC joint $(\mathrm{p}<0.01)$. With the increase in the walkway gradient from $5^{\circ}$ to $10^{\circ}$, the peak forces in three directions increased during uphill pushing, whereas during downhill pulling, these forces decreased with the increase in the walkway gradient from $5^{\circ}$ to $10^{\circ}$. A significant load weight by direction of force application interaction effect was observed for the loading of the SC joint in the three directions $(\mathrm{p}<0.05)$. During uphill pushing, increase in the load weight caused a relatively higher increase in the lateral and compression forces at the SC joint than downhill pulling. A reverse pattern was observed in the anterior-posterior force direction, where increase in the load weight caused a relatively higher increase in the AP shear forces acting at the SC joint during downhill pulling than uphill pushing. 

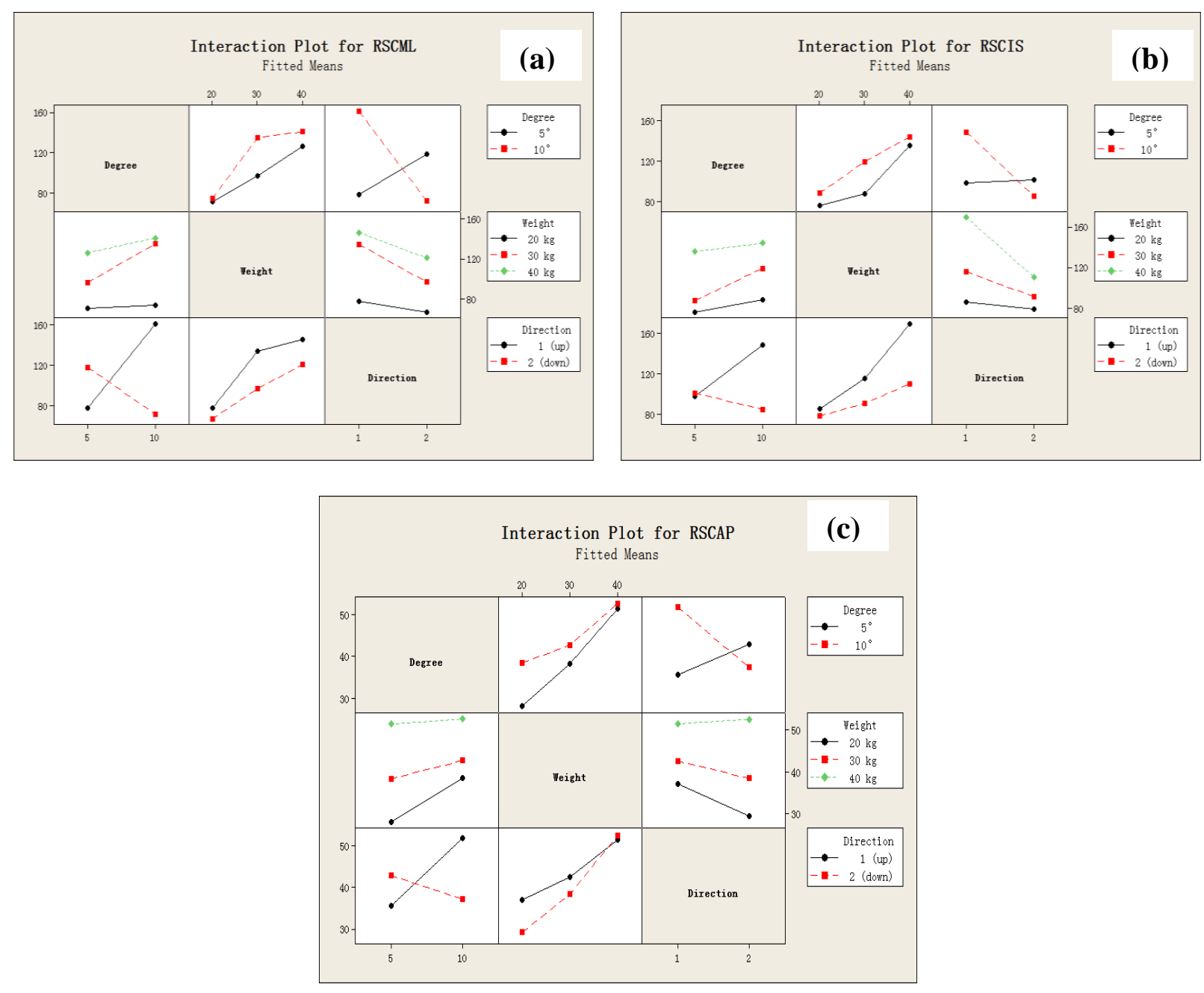

Figure 6.12: Two-way interaction plots for the loading of the sternoclavicular (SC) joint in three directions: (a) RSC-ML; (b) RSC-PD; (c) RSC-AP

\subsubsection{Three-way interaction effect and Tukey HSD analysis}

The dataset was unstacked by the walkway gradient $\left(5^{\circ}\right.$ and $\left.10^{\circ}\right)$ to facilitate the threeway interaction effect interpretation (Figure 6.13). A three-way interaction effect between walkway gradient, load weight, and direction of force exertion was significant for the peak forces acting at the SC joint in the three directions $(\mathrm{p}<0.01)$ (Figures $6.14-6.16)$.

At the walkway gradient of $5^{\circ}$, increase in the load weight increased the peak compression, lateral and anterior-posterior shear forces during downhill pulling. During uphill pushing, increase in the load weight increased the peak compression and anterior-posterior shear forces. Results of the All-pairwise comparison test showed that during downhill pulling, the mean of peak forces in three directions at the $40 \mathrm{~kg}$ weight condition was different from the 30 
$\mathrm{kg}$ weight condition. In addition to that, the $30 \mathrm{~kg}$ weight condition caused larger forces in the lateral and AP shear directions than the $20 \mathrm{~kg}$ weight condition during downhill pulling. The mean of peak compression and AP shear forces at $40 \mathrm{~kg}$ weight condition was different from 20 $\mathrm{kg}$ weight condition during downhill pulling as well as uphill pushing. A comparison between the direction of force exertion indicates that the mean of peak lateral forces during downhill pulling at $30 \mathrm{~kg}$ and $40 \mathrm{~kg}$ weight conditions were higher than uphill pushing. Additionally, the mean of peak shear forces in the anterior-posterior direction at the $40 \mathrm{~kg}$ weight condition was larger than uphill pushing.

On the $10^{\circ}$ ramp, during uphill pushing, the mean of peak lateral forces at $30 \mathrm{~kg}$ and 40 $\mathrm{kg}$ weight conditions was different from the $20 \mathrm{~kg}$ weight condition. A coherent trend was observed for the mean of peak compression forces which increased with the increase in the load weight during uphill pushing. The mean of peak shear forces in the anterior-posterior direction at the $40 \mathrm{~kg}$ weight condition was larger than the $20 \mathrm{~kg}$ and $30 \mathrm{~kg}$ weight conditions during uphill pushing. No significant difference was observed between the three weight conditions during downhill pulling. A between direction of force application comparison shows that the mean of peak forces in the three directions during uphill pushing at $30 \mathrm{~kg}$ and $40 \mathrm{~kg}$ weight conditions was larger than downhill pulling.

A between gradient comparison shows that the mean of peak compression, lateral and anterior-posterior shear forces during pushing the load weight of $40 \mathrm{~kg}$ uphill was higher at the $10^{\circ}$ ramp than the $5^{\circ}$ ramp condition. 

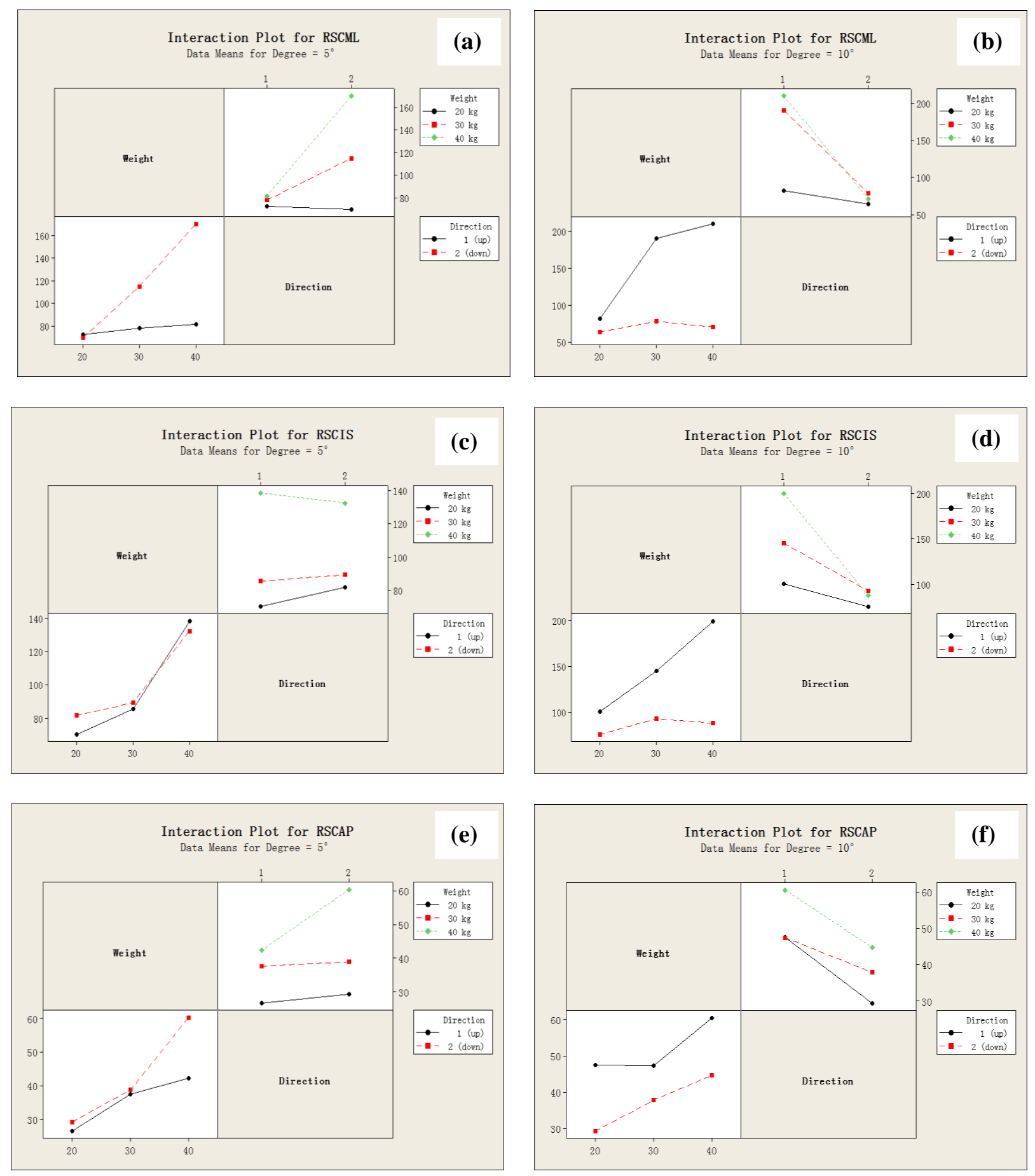

Figure 6.13: Three-way interaction plots for the loading of the sternoclavicular (SC) joint unstacked by the walkway gradient (degree) factor 


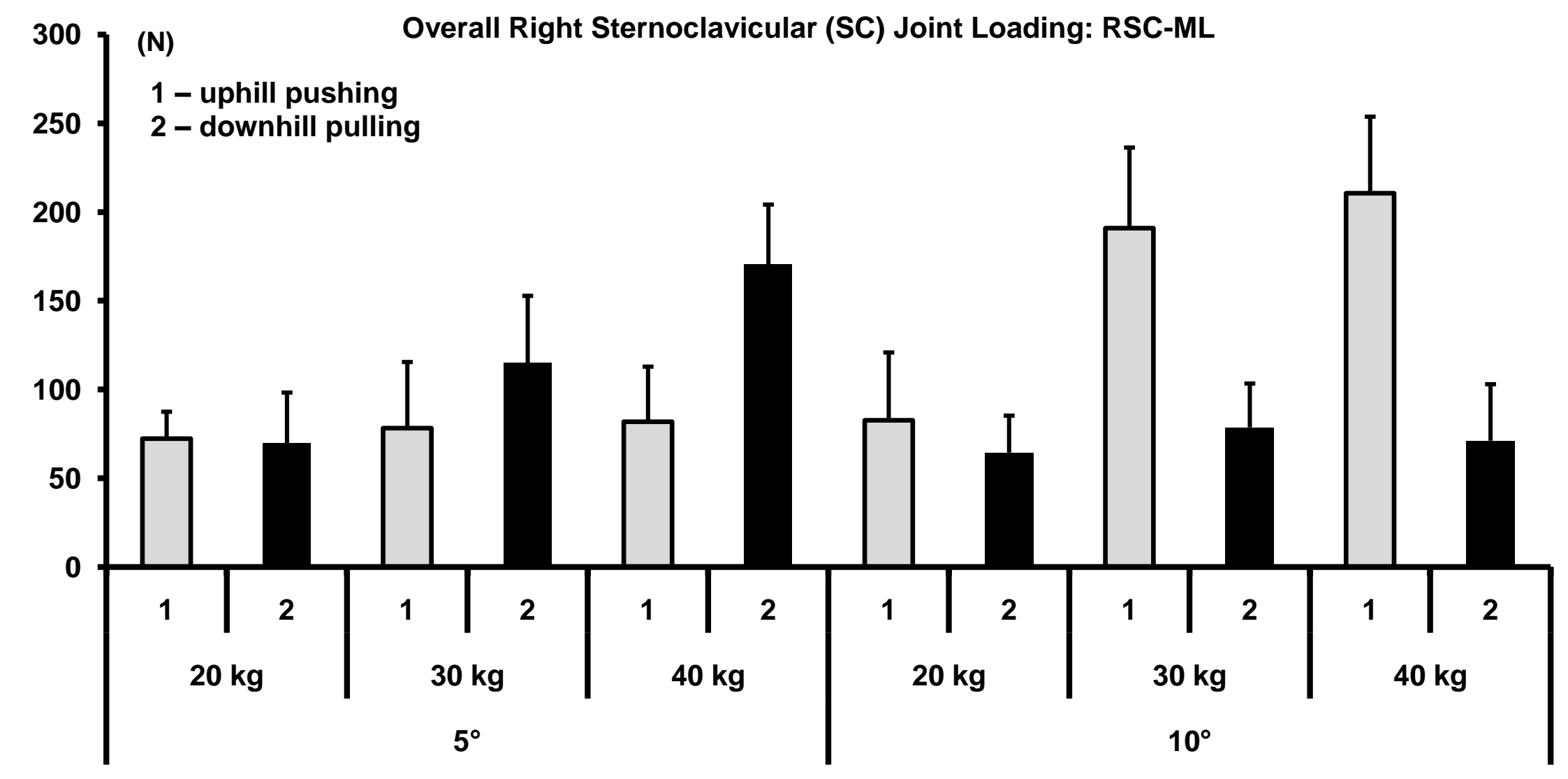

\begin{tabular}{|c|c|c|c|c|c|c|c|c|c|c|c|c|c|c|c|c|c|c|}
\hline \multicolumn{6}{|c|}{$5^{\circ}$} & \multicolumn{6}{|c|}{$10^{\circ}$} & \multirow{2}{*}{\multicolumn{7}{|c|}{$\mathrm{p}$-value }} \\
\hline \multicolumn{2}{|c|}{$20 \mathrm{~kg}$} & \multicolumn{2}{|c|}{$30 \mathrm{~kg}$} & \multicolumn{2}{|c|}{$40 \mathrm{~kg}$} & \multicolumn{2}{|c|}{$20 \mathrm{~kg}$} & \multicolumn{2}{|c|}{$30 \mathrm{~kg}$} & \multicolumn{2}{|c|}{$40 \mathrm{~kg}$} & & & & & & & \\
\hline 1 & 2 & 1 & 2 & 1 & 2 & 1 & 2 & 1 & 2 & 1 & 2 & $A^{*}$ & B & $\mathrm{C}$ & $A \times B$ & $\mathrm{~A} \times \mathrm{C}$ & $\mathrm{B} \times \mathrm{C}$ & $\mathrm{A} \times \mathrm{B} \times \mathrm{C}$ \\
\hline $\begin{array}{c}72 \\
(15)\end{array}$ & $\begin{array}{c}70 \\
(29)\end{array}$ & $\begin{array}{c}78 \\
(37)\end{array}$ & $\begin{array}{l}115 \\
(38)\end{array}$ & $\begin{array}{c}82 \\
(31)\end{array}$ & $\begin{array}{l}171 \\
(34)\end{array}$ & $\begin{array}{c}83 \\
(38)\end{array}$ & $\begin{array}{c}65 \\
(21)\end{array}$ & $\begin{array}{l}191 \\
(45)\end{array}$ & $\begin{array}{c}79 \\
(25)\end{array}$ & $\begin{array}{l}211 \\
(43)\end{array}$ & $\begin{array}{c}71 \\
(32)\end{array}$ & $<0.01$ & $<0.01$ & $<0.01$ & 0.01 & $<0.01$ & 0.04 & $<0.01$ \\
\hline
\end{tabular}

Figure 6.14: Peak force acting at the sternoclavicular (SC) joint in the medio-lateral direction during uphill pushing and downhill pulling 


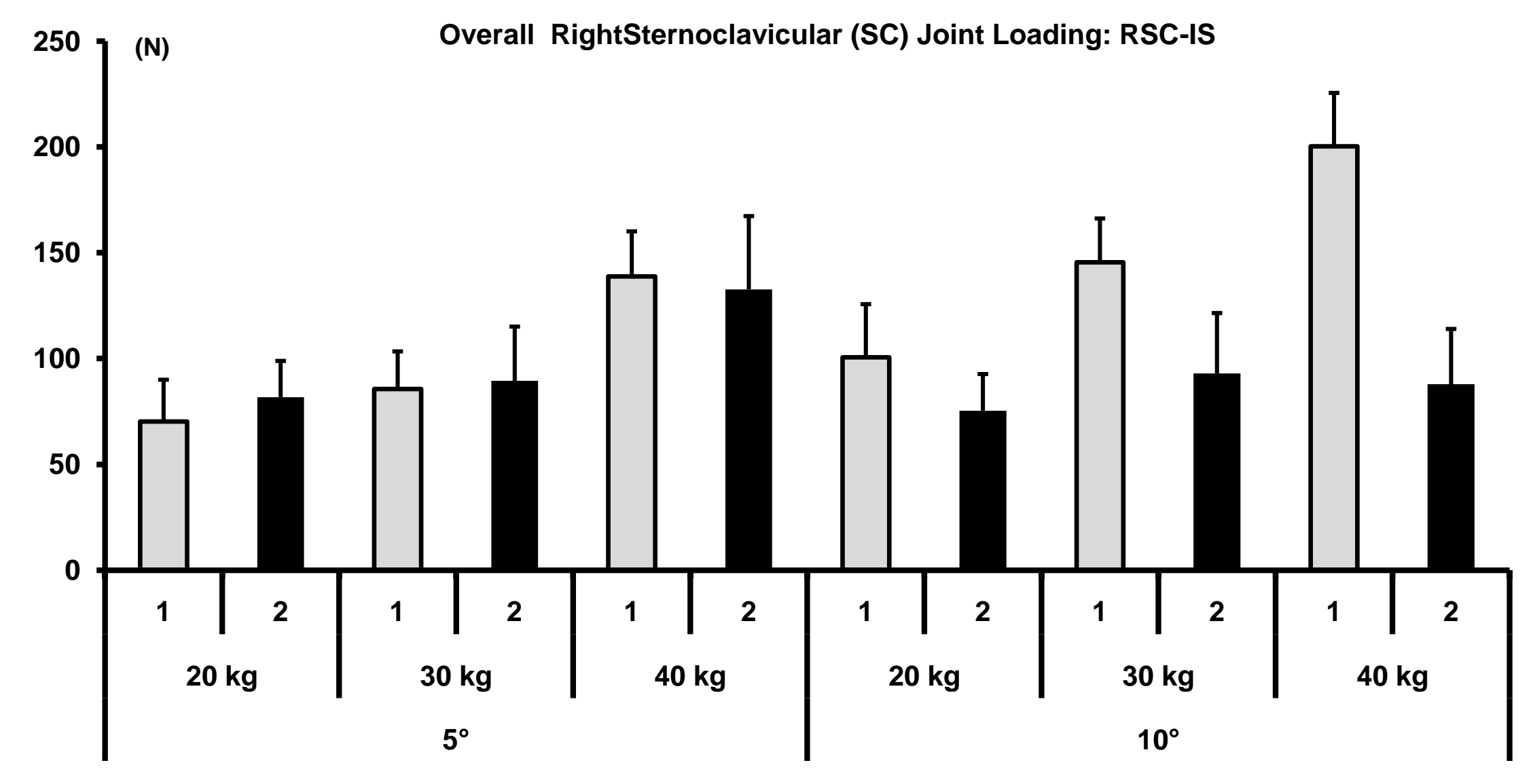

\begin{tabular}{|c|c|c|c|c|c|c|c|c|c|c|c|c|c|c|c|c|c|c|}
\hline \multicolumn{6}{|c|}{$5^{\circ}$} & \multicolumn{6}{|c|}{$10^{\circ}$} & \multirow{2}{*}{\multicolumn{7}{|c|}{ p-value }} \\
\hline \multicolumn{2}{|c|}{$20 \mathrm{~kg}$} & \multicolumn{2}{|c|}{$30 \mathrm{~kg}$} & \multicolumn{2}{|c|}{$40 \mathrm{~kg}$} & \multicolumn{2}{|c|}{$20 \mathrm{~kg}$} & \multicolumn{2}{|c|}{$30 \mathrm{~kg}$} & \multicolumn{2}{|c|}{$40 \mathrm{~kg}$} & & & & & & & \\
\hline 1 & 2 & 1 & 2 & 1 & 2 & 1 & 2 & 1 & 2 & 1 & 2 & $\mathrm{~A}^{*}$ & B & $\mathrm{C}$ & $A \times B$ & $\mathrm{~A} \times \mathrm{C}$ & $\mathrm{B} \times \mathrm{C}$ & $\mathrm{A} \times \mathrm{B} \times \mathrm{C}$ \\
\hline $\begin{array}{c}70 \\
(20)\end{array}$ & $\begin{array}{c}82 \\
(17)\end{array}$ & $\begin{array}{c}86 \\
(18)\end{array}$ & $\begin{array}{c}90 \\
(26)\end{array}$ & $\begin{array}{l}139 \\
(21)\end{array}$ & $\begin{array}{l}133 \\
\text { (35) }\end{array}$ & $\begin{array}{l}101 \\
(25)\end{array}$ & $\begin{array}{c}75 \\
(17)\end{array}$ & $\begin{array}{l}146 \\
(21)\end{array}$ & $\begin{array}{c}93 \\
(29)\end{array}$ & $\begin{array}{l}200 \\
(25)\end{array}$ & $\begin{array}{c}88 \\
(26)\end{array}$ & $<0.01$ & $<0.01$ & $<0.01$ & 0.01 & $<0.01$ & $<0.01$ & $<0.01$ \\
\hline
\end{tabular}

Figure 6.15: Peak force acting at the sternoclavicular (SC) joint in the inferior-superior direction during uphill pushing and downhill pulling 


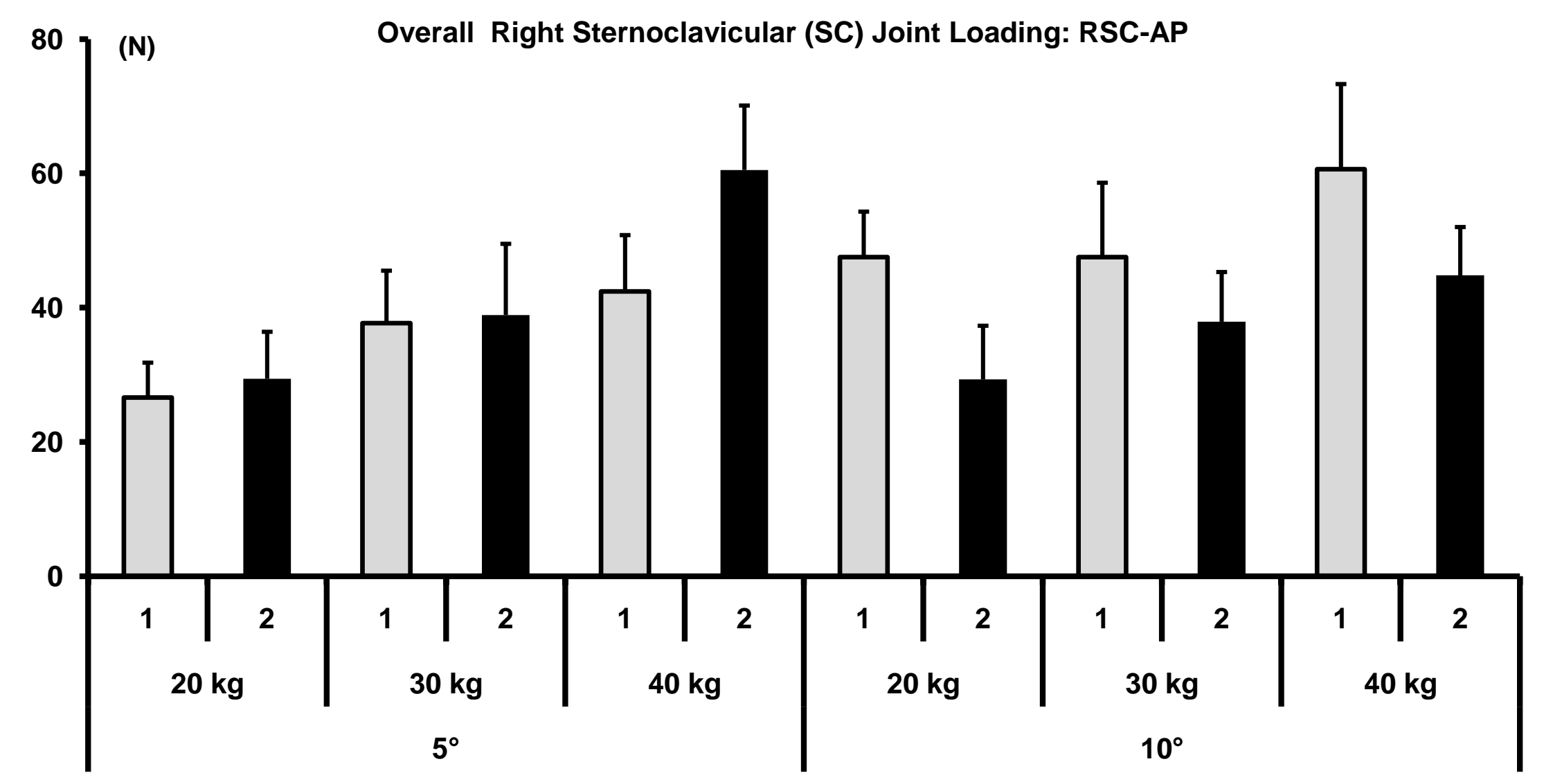

\begin{tabular}{|c|c|c|c|c|c|c|c|c|c|c|c|c|c|c|c|c|c|c|}
\hline \multicolumn{6}{|c|}{$5^{\circ}$} & \multicolumn{6}{|c|}{$10^{\circ}$} & \multirow{2}{*}{\multicolumn{7}{|c|}{ p-value }} \\
\hline \multicolumn{2}{|c|}{$20 \mathrm{~kg}$} & \multicolumn{2}{|c|}{$30 \mathrm{~kg}$} & \multicolumn{2}{|c|}{$40 \mathrm{~kg}$} & \multicolumn{2}{|c|}{$20 \mathrm{~kg}$} & \multicolumn{2}{|c|}{$30 \mathrm{~kg}$} & \multicolumn{2}{|c|}{$40 \mathrm{~kg}$} & & & & & & & \\
\hline 1 & 2 & 1 & 2 & 1 & 2 & 1 & 2 & 1 & 2 & 1 & 2 & $\mathrm{~A}^{*}$ & B & $\mathrm{C}$ & $\mathrm{A} \times \mathrm{B}$ & $\mathrm{A} \times \mathrm{C}$ & $\mathrm{B} \times \mathrm{C}$ & $\mathrm{A} \times \mathrm{B} \times \mathrm{C}$ \\
\hline $\begin{array}{l}27 \\
(5)\end{array}$ & $\begin{array}{l}29 \\
(7)\end{array}$ & $\begin{array}{c}38 \\
(8)\end{array}$ & $\begin{array}{c}39 \\
(11)\end{array}$ & $\begin{array}{l}42 \\
(8)\end{array}$ & $\begin{array}{c}61 \\
(10)\end{array}$ & $\begin{array}{l}48 \\
(7)\end{array}$ & $\begin{array}{l}29 \\
(8)\end{array}$ & $\begin{array}{c}48 \\
(11)\end{array}$ & $\begin{array}{l}38 \\
(7)\end{array}$ & $\begin{array}{c}61 \\
(13)\end{array}$ & $\begin{array}{l}45 \\
(7)\end{array}$ & $<0.01$ & $<0.01$ & $<0.01$ & $<0.01$ & $<0.01$ & $<0.01$ & $<0.01$ \\
\hline
\end{tabular}

$\mathrm{A}=$ walkway gradient; $\mathrm{B}=$ load weight $\mathrm{C}=$ direction of force application

Figure 6.16: Peak force acting at the sternoclavicular (SC) joint in the anterior-posterior direction during uphill pushing and downhill pulling 


\subsubsection{Shoulder joint loading at acromioclavicular joint}

\subsubsection{Main effects}

A general trend in the data indicate that the peak forces in three directions acting at the acromioclavicular (AC) joint increased with the increase in the load weight and walkway gradient (Figure 6.17). The comparison between two directions of force application shows that uphill pushing caused larger joint forces than downhill pulling. Statistically, the main effect of load weight, walkway gradient, and direction of force exertion on the peak AC joint forces in three directions was significant (all $\mathrm{p}<0.01$ ).
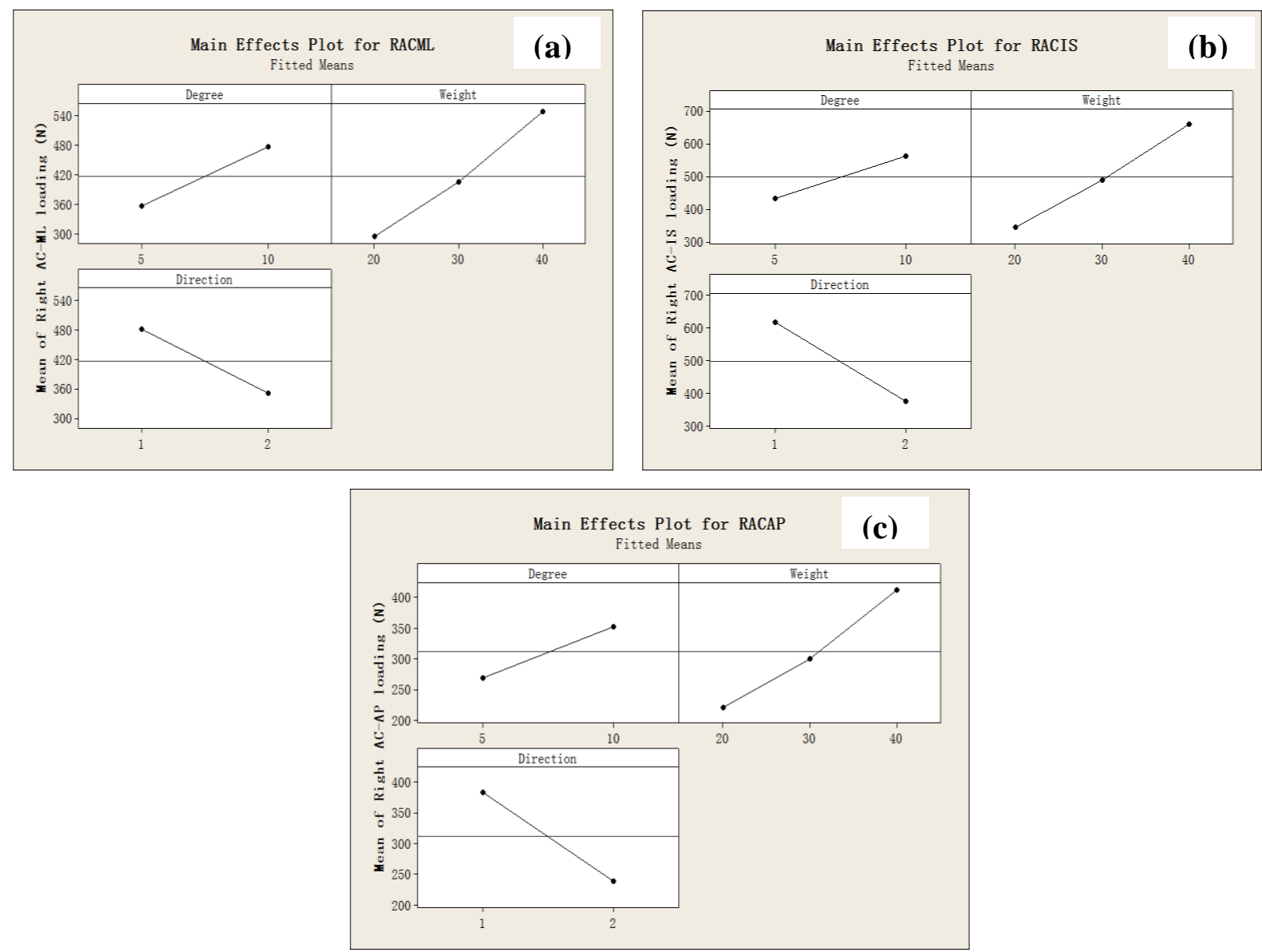

Figure 6.17: Main effect plots for the loading of the acromioclavicular (AC) joint in three directions ((a) RAC-ML; (b) RAC-IS; (c) RAC-AP) by the walkway gradient, load weight, and direction of force application

\subsubsection{Two-way interaction effects}

The interaction effect of the walkway gradient by load weight was significant for the peak shear force in the anterior-posterior direction at the AC joint $(\mathrm{p}=0.01)$. The 
interaction effect was ordinal, indicating that the increase in the walkway gradient at 40 $\mathrm{kg}$ weight caused a relatively higher increase in the peak AP shear force than at $20 \mathrm{~kg}$ and $30 \mathrm{~kg}$ weight conditions (Figure 6.18 (c)). A disordinal interaction effect between walkway gradient and direction of force application was observed for the peak forces in three directions $(\mathrm{p}<0.01)$. With the increase in the walkway gradient from $5^{\circ}$ to $10^{\circ}$, the peak forces in three directions increased during uphill pushing, whereas during downhill pulling these forces decreased with the increase in the walkway gradient from $5^{\circ}$ to $10^{\circ}$. The effect of load weight and direction of force exertion was ordinal and significant for the peak forces in three directions $(\mathrm{p}<0.01)$. It was observed that the increase in the load weight caused a relatively higher increase in the loading at the AC joint during uphill pushing than downhill pulling activities.
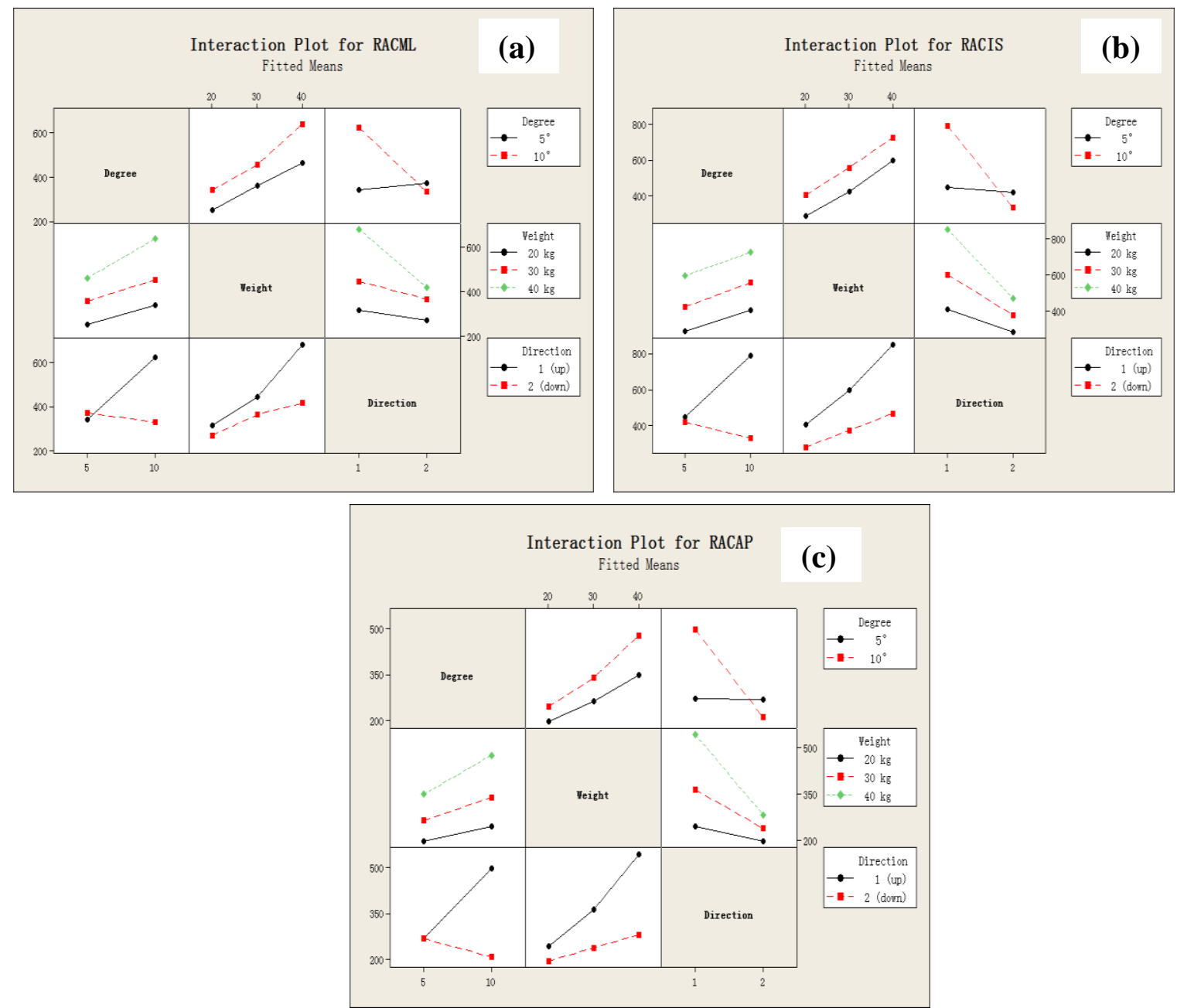

Figure 6.18: Two-way interaction plots for the loading of the acromioclavicular (AC) joint in three directions: (a) RAC-ML; (b) RAC-IS; (c) RAC-AP 


\subsubsection{Three-way interaction effect}

A three-way interaction effect of walkway gradient by load weight by direction of force exertion was significant for the peak forces acting at the AC joint in three directions $(\mathrm{p}<0.01)$ (Figures $6.20-6.22$ ).

As shown in Figure 6.19 (a) (c) (e), at the ramp of $5^{\circ}$, increase in the load weight increased the peak compression and shear forces during uphill pushing as well as downhill pulling. Results of the All-pairwise comparison test indicated that the mean of peak forces in three directions at $40 \mathrm{~kg}$ weight condition was different from $20 \mathrm{~kg}$ weight condition during uphill pushing and downhill pulling. In addition, the mean of peak compression force in the inferior-superior direction at $40 \mathrm{~kg}$ weight condition was different from $30 \mathrm{~kg}$ weight condition during uphill pushing.

As can be seen in Figure 6.19 (b) (d) (f), at the ramp of $10^{\circ}$, the mean of peak forces in three directions increased with the increase in the load weight during uphill pushing activities. Results of the All-pairwise comparison test indicated that the mean of peak compression, lateral and anterior-posterior shear forces increased with the corresponding three weight conditions. No significant difference was observed between the three weight conditions during downhill pulling. A comparison between directions of force exertion showed that the mean of peak forces acting at the $\mathrm{AC}$ joint in three directions during uphill pushing at $30 \mathrm{~kg}$ and $40 \mathrm{~kg}$ weight conditions was higher than downhill pulling. This direction difference was also observed for the mean of peak compression force in the inferior-superior direction at $20 \mathrm{~kg}$ weight condition.

A between walkway gradient comparison showed that the mean of peak forces in three directions during uphill pushing at $30 \mathrm{~kg}$ and $40 \mathrm{~kg}$ weight conditions was higher at the ramp of $10^{\circ}$ than $5^{\circ}$. The same trend was also observed at $20 \mathrm{~kg}$ weight condition for the mean of peak forces in lateral and compression forces. 

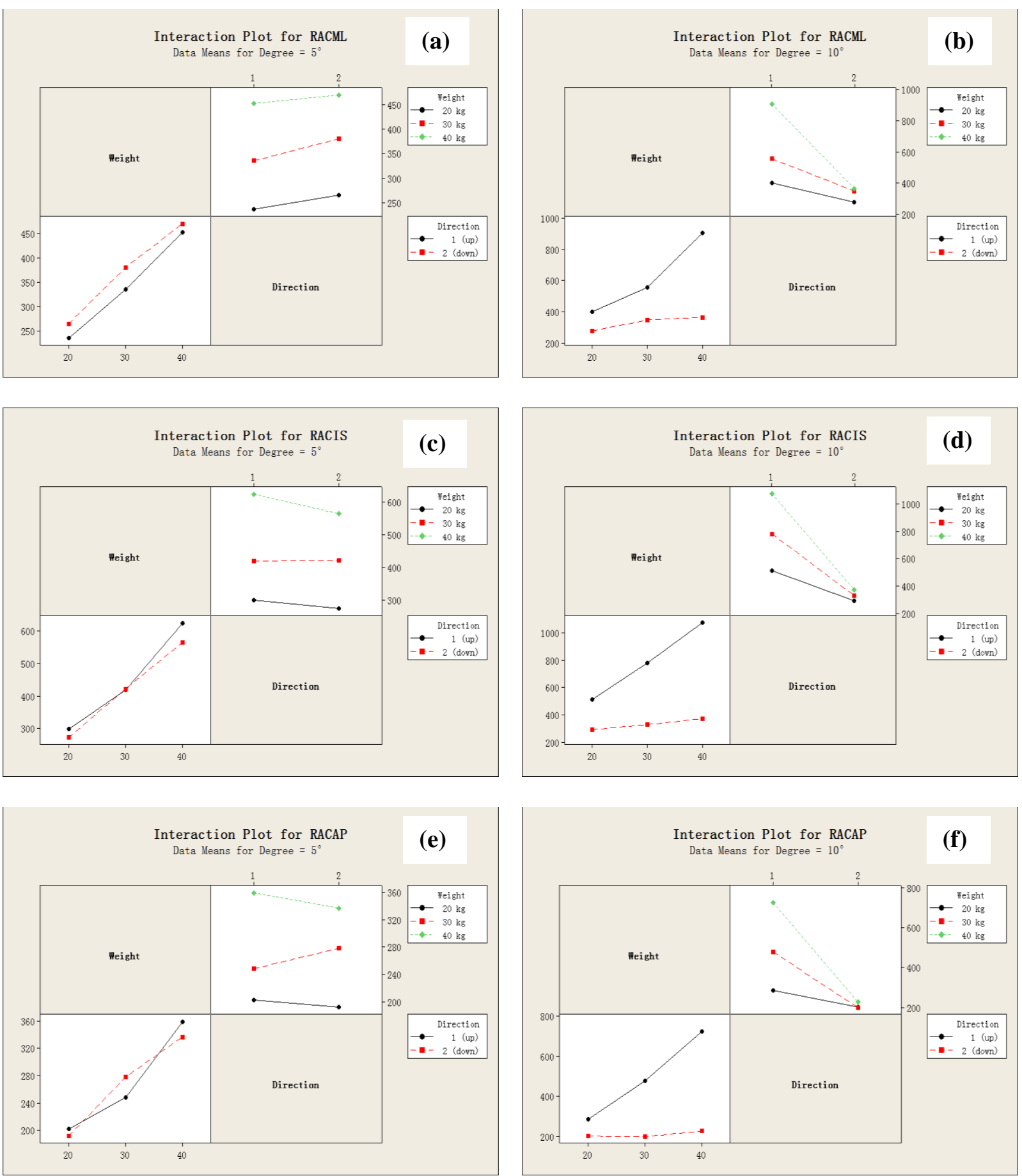

Figure 6.19: Three-way interaction plots for the loading of the acromioclavicular (AC) joint unstacked by the walkway gradient (degree) factor 


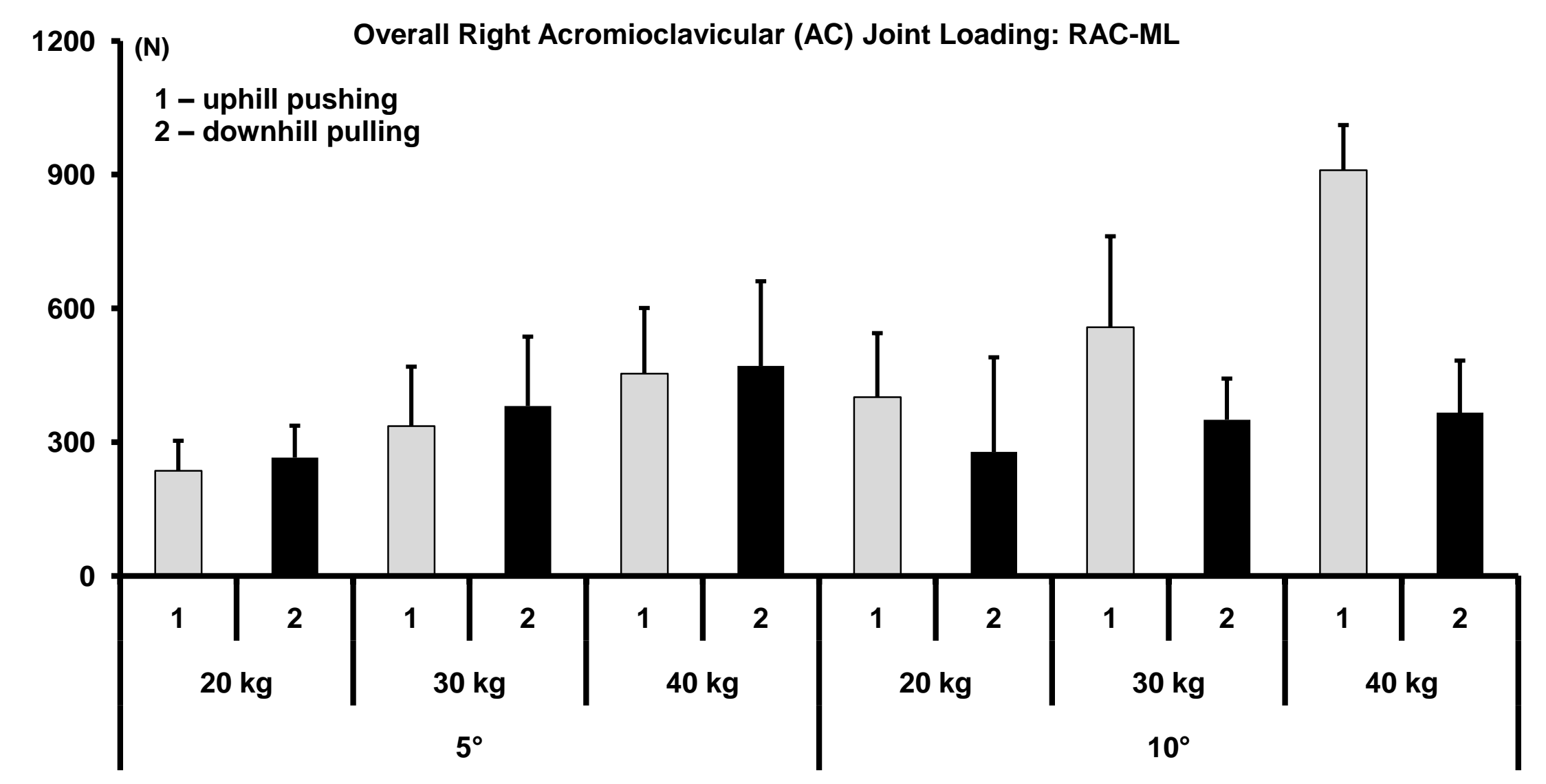

\begin{tabular}{|c|c|c|c|c|c|c|c|c|c|c|c|c|c|c|c|c|c|c|}
\hline \multicolumn{6}{|c|}{$5^{\circ}$} & \multicolumn{6}{|c|}{$10^{\circ}$} & \multirow{2}{*}{\multicolumn{7}{|c|}{$\mathrm{p}$-value }} \\
\hline \multicolumn{2}{|c|}{$20 \mathrm{~kg}$} & \multicolumn{2}{|c|}{$30 \mathrm{~kg}$} & \multicolumn{2}{|c|}{$40 \mathrm{~kg}$} & \multicolumn{2}{|c|}{$20 \mathrm{~kg}$} & \multicolumn{2}{|c|}{$30 \mathrm{~kg}$} & \multicolumn{2}{|c|}{$40 \mathrm{~kg}$} & & & & & & & \\
\hline 1 & 2 & 1 & 2 & 1 & 2 & 1 & 2 & 1 & 2 & 1 & 2 & $\mathrm{~A}^{*}$ & $\mathrm{~B}$ & $\mathrm{C}$ & $\mathrm{A} \times \mathrm{B}$ & $\mathrm{A} \times \mathrm{C}$ & $\mathrm{B} \times \mathrm{C}$ & $\mathrm{A} \times \mathrm{B} \times \mathrm{C}$ \\
\hline $\begin{array}{l}236 \\
(67)\end{array}$ & $\begin{array}{l}265 \\
(72) \\
\end{array}$ & $\begin{array}{c}336 \\
(133)\end{array}$ & $\begin{array}{c}381 \\
(156)\end{array}$ & $\begin{array}{c}454 \\
(147)\end{array}$ & $\begin{array}{c}471 \\
(190)\end{array}$ & $\begin{array}{c}401 \\
(143)\end{array}$ & $\begin{array}{c}278 \\
(212)\end{array}$ & $\begin{array}{c}558 \\
(204)\end{array}$ & $\begin{array}{l}350 \\
(92) \\
\end{array}$ & $\begin{array}{c}910 \\
(101)\end{array}$ & $\begin{array}{c}366 \\
(117)\end{array}$ & $<0.01$ & $<0.01$ & $<0.01$ & 0.07 & $<0.01$ & $<0.01$ & $<0.01$ \\
\hline
\end{tabular}

$\mathrm{A}=$ walkway gradient; $\mathrm{B}=$ load weight $\mathrm{C}=$ direction of force application

Figure 6.20: Peak force acting at the acromioclavicualr (AC) joint in the medio-lateral direction during uphill pushing and downhill pulling 


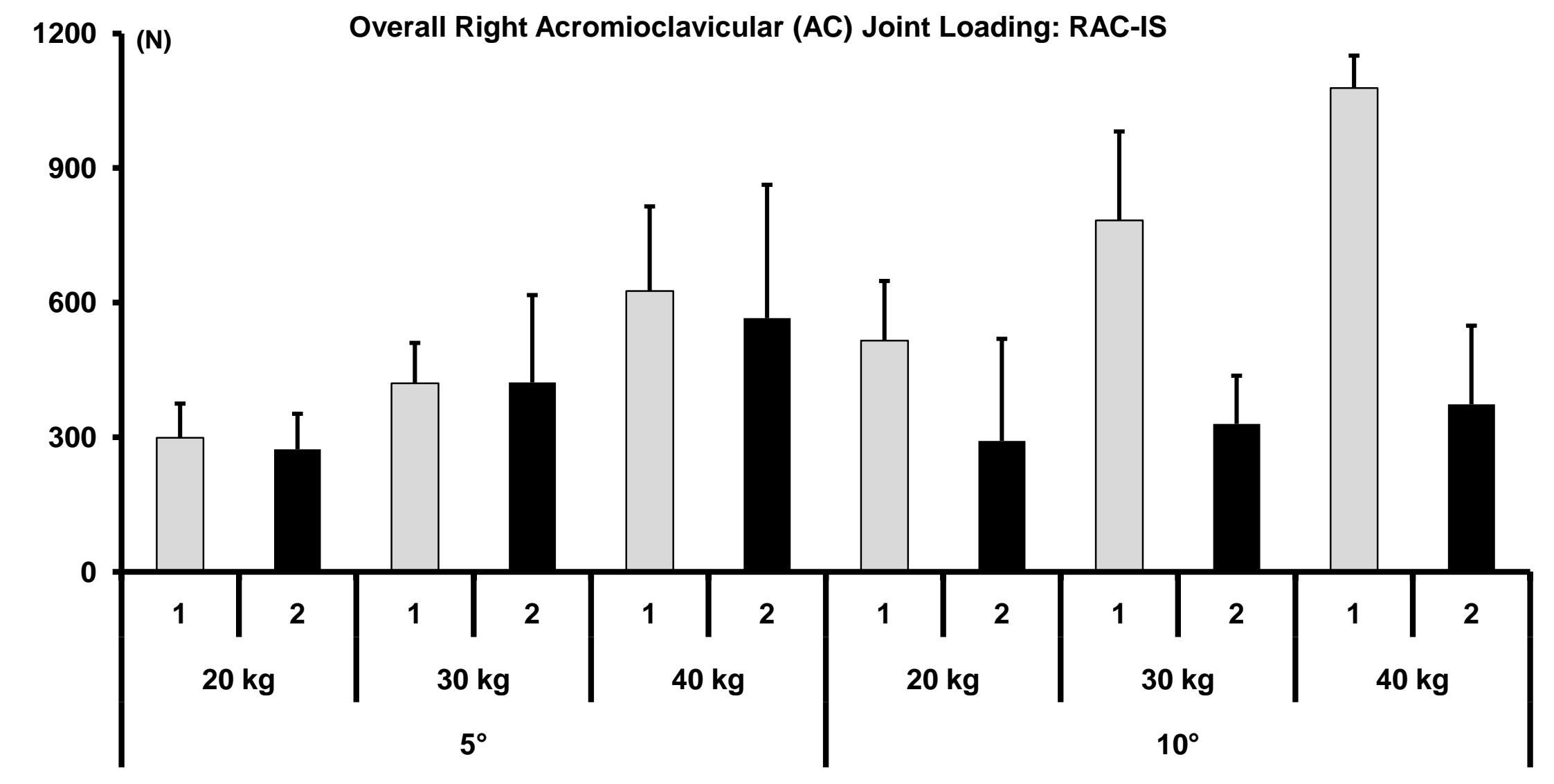

\begin{tabular}{|c|c|c|c|c|c|c|c|c|c|c|c|c|c|c|c|c|c|c|}
\hline \multicolumn{6}{|c|}{$5^{\circ}$} & \multicolumn{6}{|c|}{$10^{\circ}$} & \multirow{2}{*}{\multicolumn{7}{|c|}{ p-value }} \\
\hline \multicolumn{2}{|c|}{$20 \mathrm{~kg}$} & \multicolumn{2}{|c|}{$30 \mathrm{~kg}$} & \multicolumn{2}{|c|}{$40 \mathrm{~kg}$} & \multicolumn{2}{|c|}{$20 \mathrm{~kg}$} & \multicolumn{2}{|c|}{$30 \mathrm{~kg}$} & \multicolumn{2}{|c|}{$40 \mathrm{~kg}$} & & & & & & & \\
\hline 1 & 2 & 1 & 2 & 1 & 2 & 1 & 2 & 1 & 2 & 1 & 2 & $\mathrm{~A}^{*}$ & B & $\mathrm{C}$ & $A \times B$ & $\mathrm{~A} \times \mathrm{C}$ & $\mathrm{B} \times \mathrm{C}$ & $\mathrm{A} \times \mathrm{B} \times \mathrm{C}$ \\
\hline $\begin{array}{l}298 \\
(77)\end{array}$ & $\begin{array}{c}273 \\
(79)\end{array}$ & $\begin{array}{l}420 \\
(90)\end{array}$ & $\begin{array}{c}422 \\
(195)\end{array}$ & $\begin{array}{c}626 \\
(189)\end{array}$ & $\begin{array}{c}565 \\
(297)\end{array}$ & $\begin{array}{c}515 \\
(133)\end{array}$ & $\begin{array}{c}292 \\
(227)\end{array}$ & $\begin{array}{c}784 \\
(198)\end{array}$ & $\begin{array}{c}330 \\
(108)\end{array}$ & $\begin{array}{c}1078 \\
(73)\end{array}$ & $\begin{array}{c}373 \\
(175)\end{array}$ & $<0.01$ & $<0.01$ & $<0.01$ & 0.95 & $<0.01$ & $<0.01$ & $<0.01$ \\
\hline
\end{tabular}

$\mathrm{A}=$ walkway gradient; $\mathrm{B}=$ load weight; $\mathrm{C}=$ direction of force application

Figure 6.21: Peak force acting at the acromioclavicualr (AC) joint in the inferior-superior direction during uphill pushing and downhill pulling 


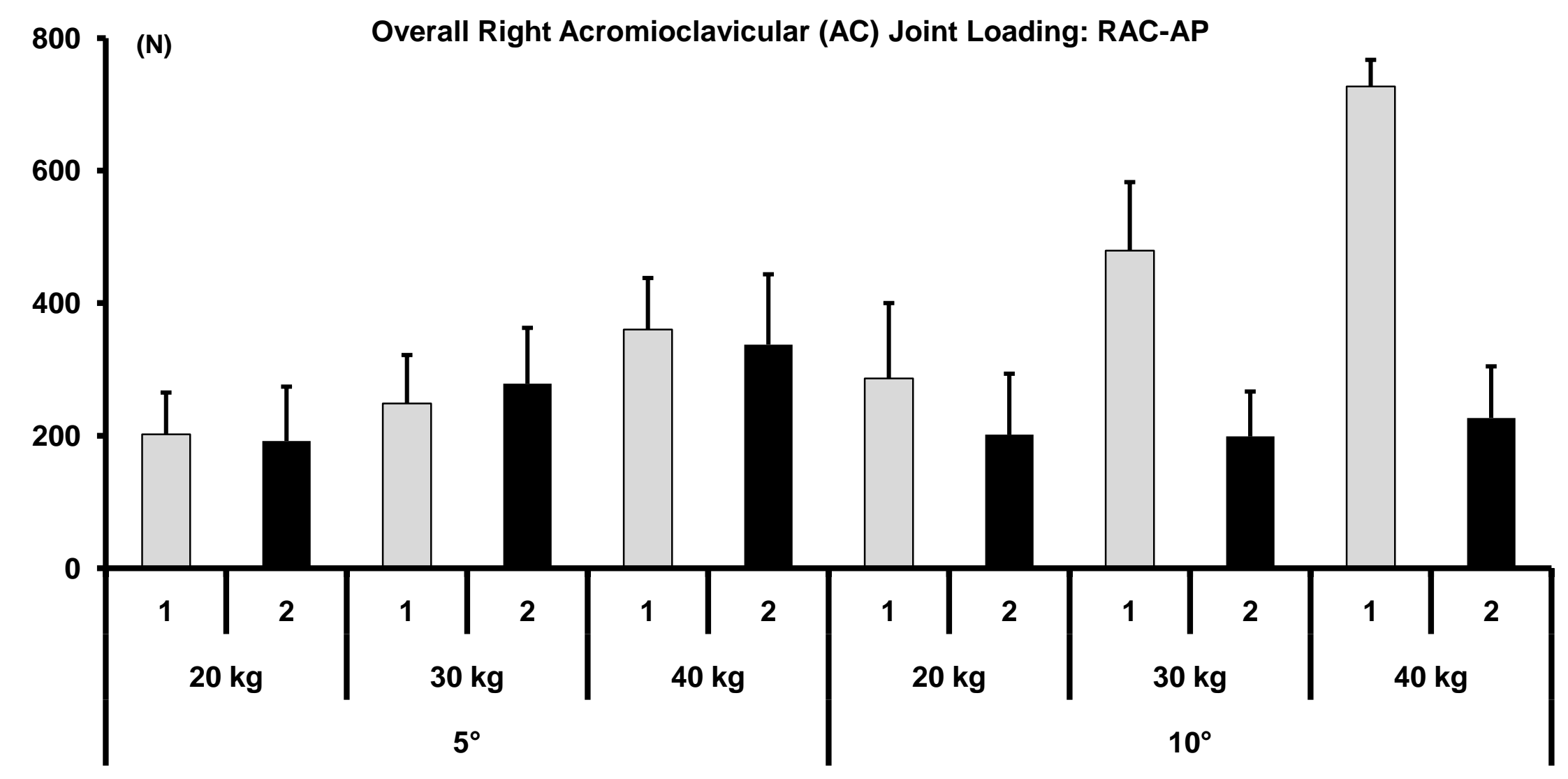

\begin{tabular}{|c|c|c|c|c|c|c|c|c|c|c|c|c|c|c|c|c|c|c|}
\hline \multicolumn{6}{|c|}{$5^{\circ}$} & \multicolumn{6}{|c|}{$10^{\circ}$} & \multirow{2}{*}{\multicolumn{7}{|c|}{$\mathrm{p}$-value }} \\
\hline \multicolumn{2}{|c|}{$20 \mathrm{~kg}$} & \multicolumn{2}{|c|}{$30 \mathrm{~kg}$} & \multicolumn{2}{|c|}{$40 \mathrm{~kg}$} & \multicolumn{2}{|c|}{$20 \mathrm{~kg}$} & \multicolumn{2}{|c|}{$30 \mathrm{~kg}$} & \multicolumn{2}{|c|}{$40 \mathrm{~kg}$} & & & & & & & \\
\hline 1 & 2 & 1 & 2 & 1 & 2 & 1 & 2 & 1 & 2 & 1 & 2 & $A^{*}$ & B & $\mathrm{C}$ & $A \times B$ & $\mathrm{~A} \times \mathrm{C}$ & $\mathrm{B} \times \mathrm{C}$ & $\mathrm{A} \times \mathrm{B} \times \mathrm{C}$ \\
\hline $\begin{array}{l}202 \\
(63)\end{array}$ & $\begin{array}{l}192 \\
(82)\end{array}$ & $\begin{array}{l}249 \\
(73)\end{array}$ & $\begin{array}{l}279 \\
(84)\end{array}$ & $\begin{array}{l}360 \\
(78)\end{array}$ & $\begin{array}{c}338 \\
(106)\end{array}$ & $\begin{array}{c}286 \\
(114)\end{array}$ & $\begin{array}{l}202 \\
(92)\end{array}$ & $\begin{array}{c}479 \\
(104)\end{array}$ & $\begin{array}{l}199 \\
(68)\end{array}$ & $\begin{array}{l}727 \\
(40)\end{array}$ & $\begin{array}{c}227 \\
(78)\end{array}$ & $<0.01$ & $<0.01$ & $<0.01$ & 0.01 & $<0.01$ & $<0.01$ & $<0.01$ \\
\hline
\end{tabular}

Figure 6.22: Peak force acting at the acromioclavicualr (AC) joint in the anterior-posterior direction during uphill pushing and downhill pulling 


\subsubsection{Shoulder joint loading at glenohumeral joint}

\subsubsection{Main effects}

In general, as shown in Figure 6.23, the peak compression and shear forces acting at the glenohumeral $(\mathrm{GH})$ joint increased with the increase in the load weight. Compared to downhill pulling, uphill pushing caused higher forces in three directions. Statistically, the main effect of walkway gradient, load weight, and direction of force application was observed in the joint forces acting at the $\mathrm{GH}$ joint in three directions (all $\mathrm{p}<0.01$ ). The magnitude of distraction shear force at the GH joint was larger than the compression force in the inferior-superior direction and shear force in the anteriorposterior direction.
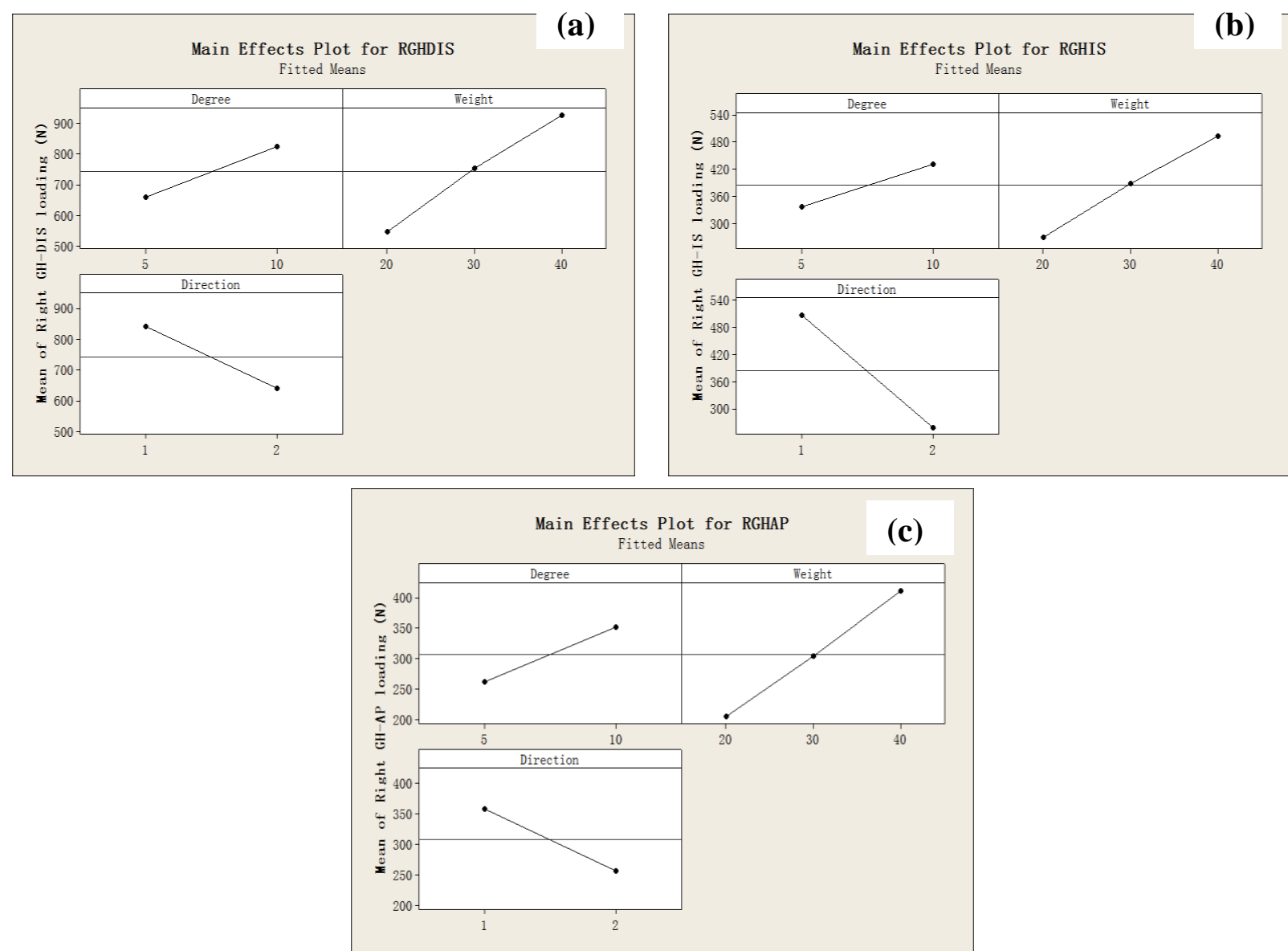

Figure 6.23: Main effect plots for the loading of the glenohumeral (GH) joint in three directions ((a) RGH-DIS; (b) RGH-IS; (c) RGH-AP) by the walkway gradient, load weight, and direction of force application 


\subsubsection{Two-way interaction effects}

A disordinal interaction effect of walkway gradient by load weight was observed for the peak shear force in the anterior-posterior direction at the GH joint $(\mathrm{p}=0.04)$. As can be seen in Figure 6.24 (c), the increase in the load weight along the walkway gradient of $10^{\circ}$ caused a relatively higher increase in the peak AP shear forces than the gradient of $5^{\circ}$. The interaction effect between walkway gradient and direction of force exertion was evident for the peak forces in three directions (all $\mathrm{p}<0.01$ ). It was observed that with the increase of walkway gradient from $5^{\circ}$ to $10^{\circ}$, peak forces in three directions increased during uphill pushing, whereas during downhill pulling, these forces decreased with the increase of walkway gradient from $5^{\circ}$ to $10^{\circ}$. Finally, an ordinal interaction effect between load weight and direction of force application was found for the peak forces at the $\mathrm{GH}$ joint in three directions (all $\mathrm{p}<0.01$ ). Corresponding to the increase in the load weight, a relatively higher increase was observed for the 3D loading of GH joint during uphill pushing than downhill pulling.
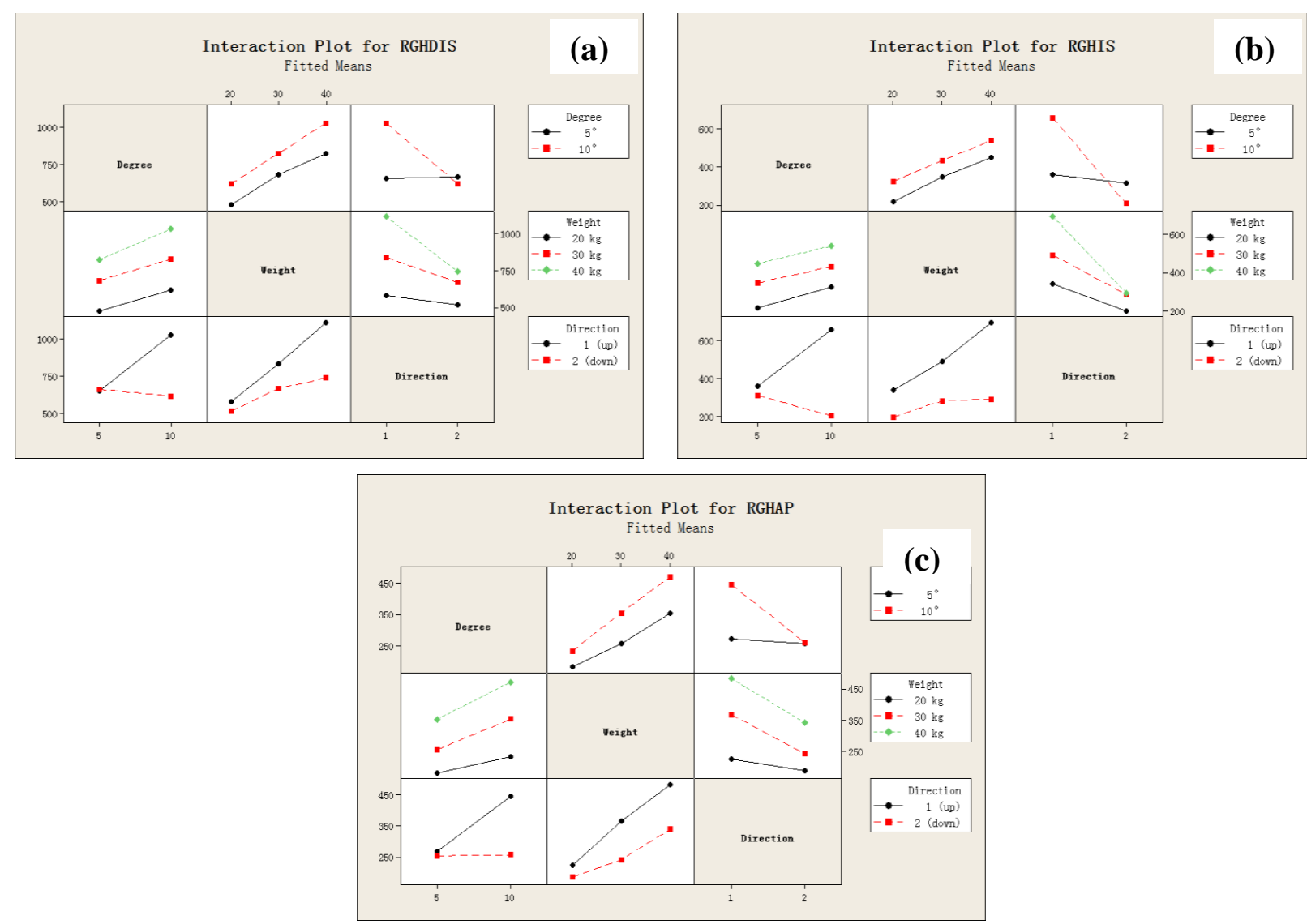

Figure 6.24: Two-way interaction plots for the loading of the glenohumeral (GH) joint in three directions: (a) RGH-DIS; (b) RGH-IS; (c) RGH-AP 


\subsubsection{Three-way interaction effect and Tukey HSD analysis}

The peak compression and shear forces acting at the GH joint was also significantly affected by the three-way interaction effect between walkway gradient, load weight, and direction of force application (all $\mathrm{p}<0.05$ ) (Figure $6.26-6.28$ ).

Figure 6.25 (a) (c) (e) shows the three-way interaction effect depending on the walkway gradient of $5^{\circ}$. Corresponding to the increase in the load weight, the peak compression and shear forces increased during uphill pushing as well as downhill pulling. Results of the All-pairwise comparison test indicated that the mean of peak forces in three directions at $40 \mathrm{~kg}$ weight condition was larger than $20 \mathrm{~kg}$ weight during uphill pushing and downhill pulling. During uphill pushing, the mean of peak distraction and anterior-posterior shear forces at the $30 \mathrm{~kg}$ weight condition was different from $20 \mathrm{~kg}$ weight condition. During the same direction of force application, the mean of peak distraction and compression (inferior-superior direction) forces at $40 \mathrm{~kg}$ weight condition was different from the $30 \mathrm{~kg}$ weight condition. On the other hand, during downhill pulling, the mean of peak distraction and compression forces at $30 \mathrm{~kg}$ weight condition was different from the $20 \mathrm{~kg}$ weight condition. Moreover, the mean of peak shear force in anterior-posterior direction at $40 \mathrm{~kg}$ weight condition was also different from the $30 \mathrm{~kg}$ weight condition during downhill pulling.

Figure 6.25 (b) (d) (f) shows the three-way interaction effect depending on the walkway gradient of $10^{\circ}$. A coherent trend was observed for the peak compression and shear forces during uphill pushing. The 3D loading of the GH joint increased with the increase in the load weight. A comparison between the directions of force exertion shows that the mean of peak forces acting at the GH joint in three directions during uphill pushing at $30 \mathrm{~kg}$ and $40 \mathrm{~kg}$ weight conditions was higher than downhill pulling. For the mean of peak compression force in the inferior-superior direction, the uphill pushing activities under the $20 \mathrm{~kg}$ weight condition caused larger forces than downhill pulling.

A between walkway gradient comparison shows that during uphill pushing, the $10^{\circ}$ ramp caused larger joint forces in three directions than the $5^{\circ}$ ramp at $30 \mathrm{~kg}$ and $40 \mathrm{~kg}$ weight conditions. Under the same conditions, a similar trend was observed for the peak distraction force and compression forces at the $20 \mathrm{~kg}$ weight condition. 

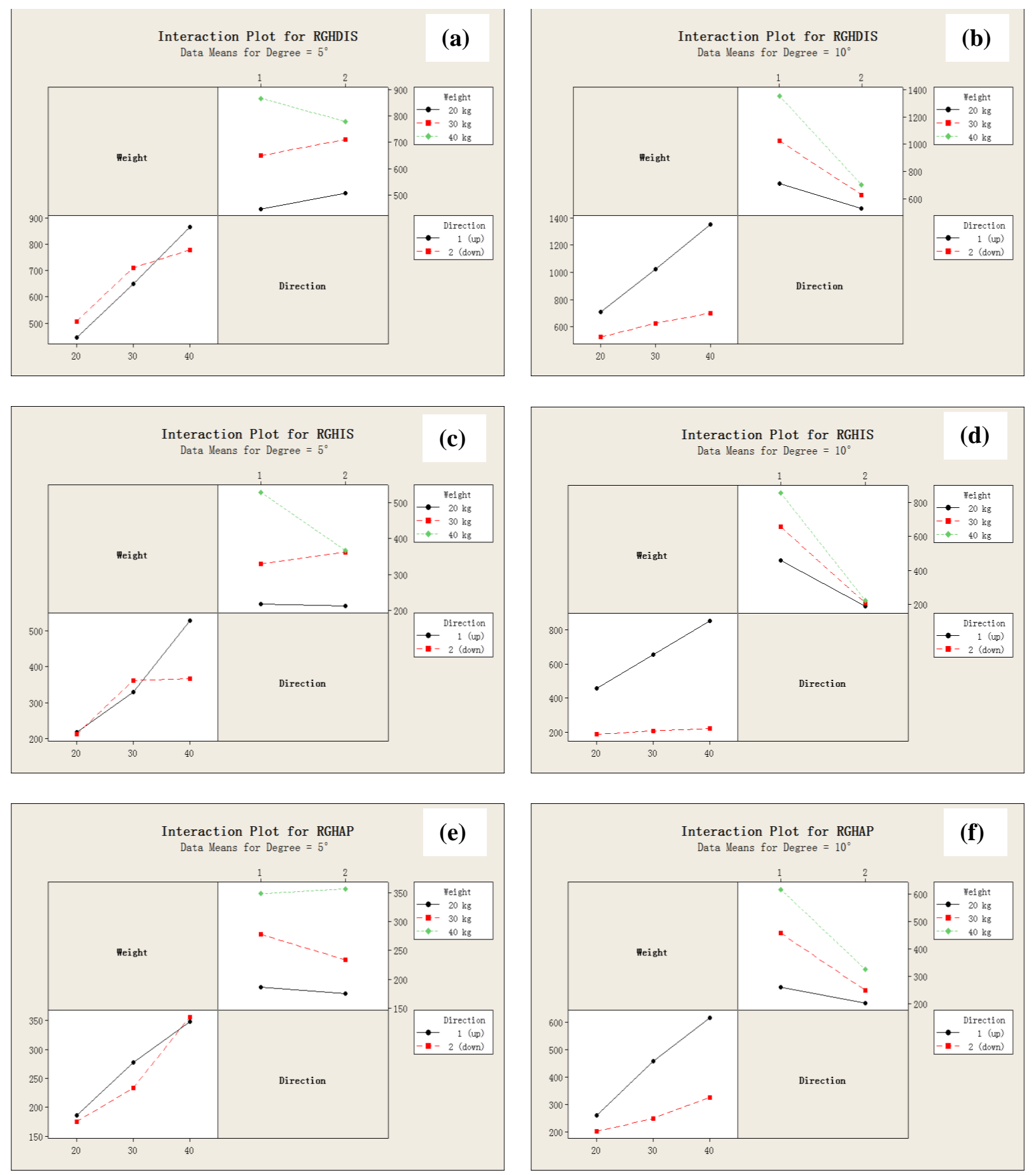

Figure 6.25: Three-way interaction plots for the loading of the glenohumeral (GH) joint unstacked by the walkway gradient (degree) factor 


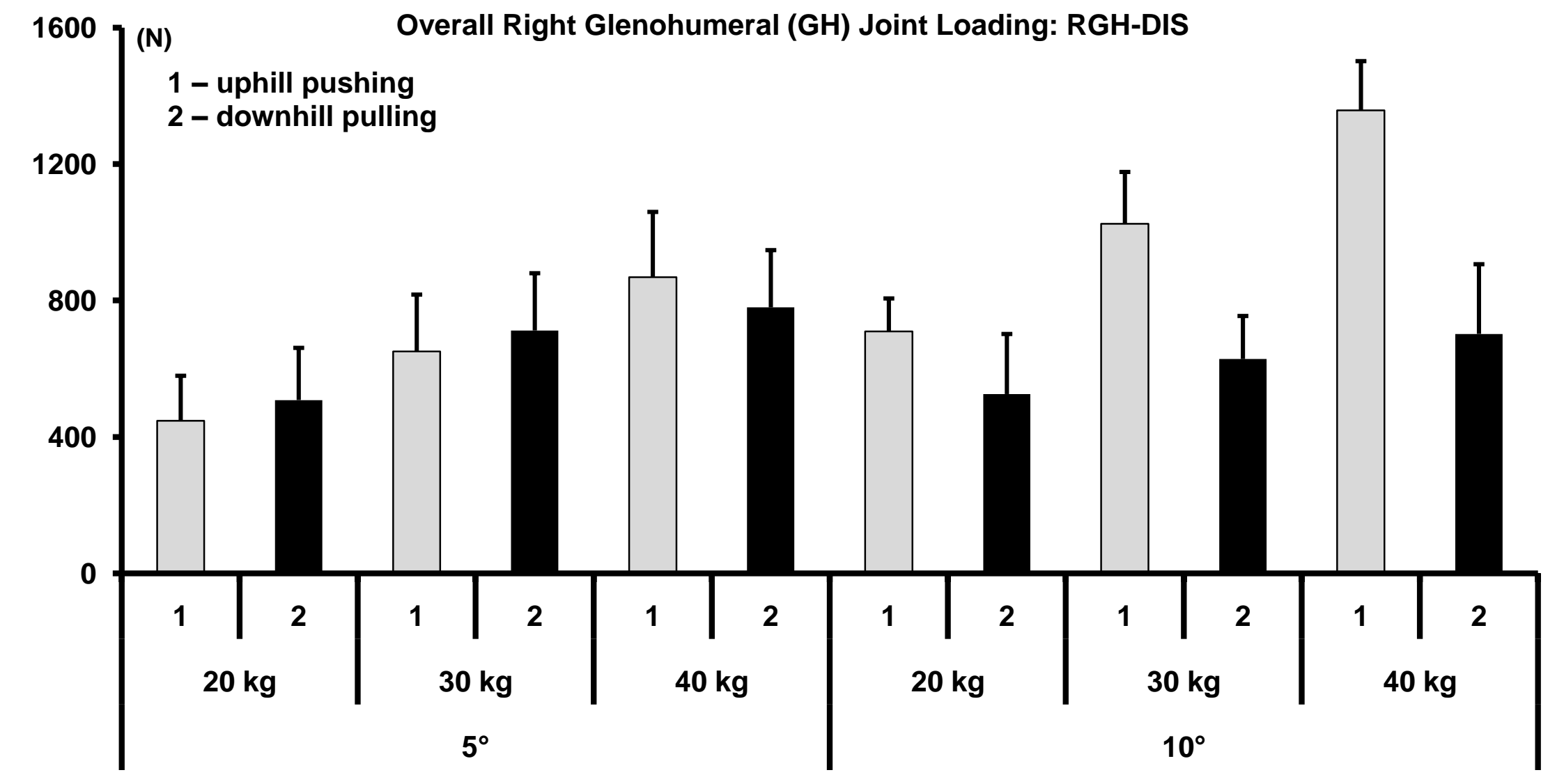

\begin{tabular}{|c|c|c|c|c|c|c|c|c|c|c|c|c|c|c|c|c|c|c|}
\hline \multicolumn{6}{|c|}{$5^{\circ}$} & \multicolumn{6}{|c|}{$10^{\circ}$} & \multirow{2}{*}{\multicolumn{7}{|c|}{ p-value }} \\
\hline \multicolumn{2}{|c|}{$20 \mathrm{~kg}$} & \multicolumn{2}{|c|}{$30 \mathrm{~kg}$} & \multicolumn{2}{|c|}{$40 \mathrm{~kg}$} & \multicolumn{2}{|c|}{$20 \mathrm{~kg}$} & \multicolumn{2}{|c|}{$30 \mathrm{~kg}$} & \multicolumn{2}{|c|}{$40 \mathrm{~kg}$} & & & & & & & \\
\hline 1 & 2 & 1 & 2 & 1 & 2 & 1 & 2 & 1 & 2 & 1 & 2 & $\mathrm{~A}^{*}$ & B & $\mathrm{C}$ & $A \times B$ & $\mathrm{~A} \times \mathrm{C}$ & $\mathrm{B} \times \mathrm{C}$ & $\mathrm{A} \times \mathrm{B} \times \mathrm{C}$ \\
\hline $\begin{array}{c}447 \\
(132)\end{array}$ & $\begin{array}{c}508 \\
(153)\end{array}$ & $\begin{array}{c}650 \\
(167)\end{array}$ & $\begin{array}{c}712 \\
(168)\end{array}$ & $\begin{array}{c}868 \\
(192)\end{array}$ & $\begin{array}{c}780 \\
(168)\end{array}$ & $\begin{array}{l}709 \\
(97)\end{array}$ & $\begin{array}{c}526 \\
(176)\end{array}$ & $\begin{array}{l}1025 \\
(152)\end{array}$ & $\begin{array}{c}628 \\
(126)\end{array}$ & $\begin{array}{l}1357 \\
(145)\end{array}$ & $\begin{array}{c}702 \\
(204)\end{array}$ & $<0.01$ & $<0.01$ & $<0.01$ & 0.44 & $<0.01$ & $<0.01$ & 0.02 \\
\hline
\end{tabular}

$\mathrm{A}=$ walkway gradient; $\mathrm{B}=$ load weight; $\mathrm{C}=$ direction of force application

Figure 6.26: Peak force acting at the glenohumeral $(\mathrm{GH})$ joint in the distraction direction during uphill pushing and downhill pulling 


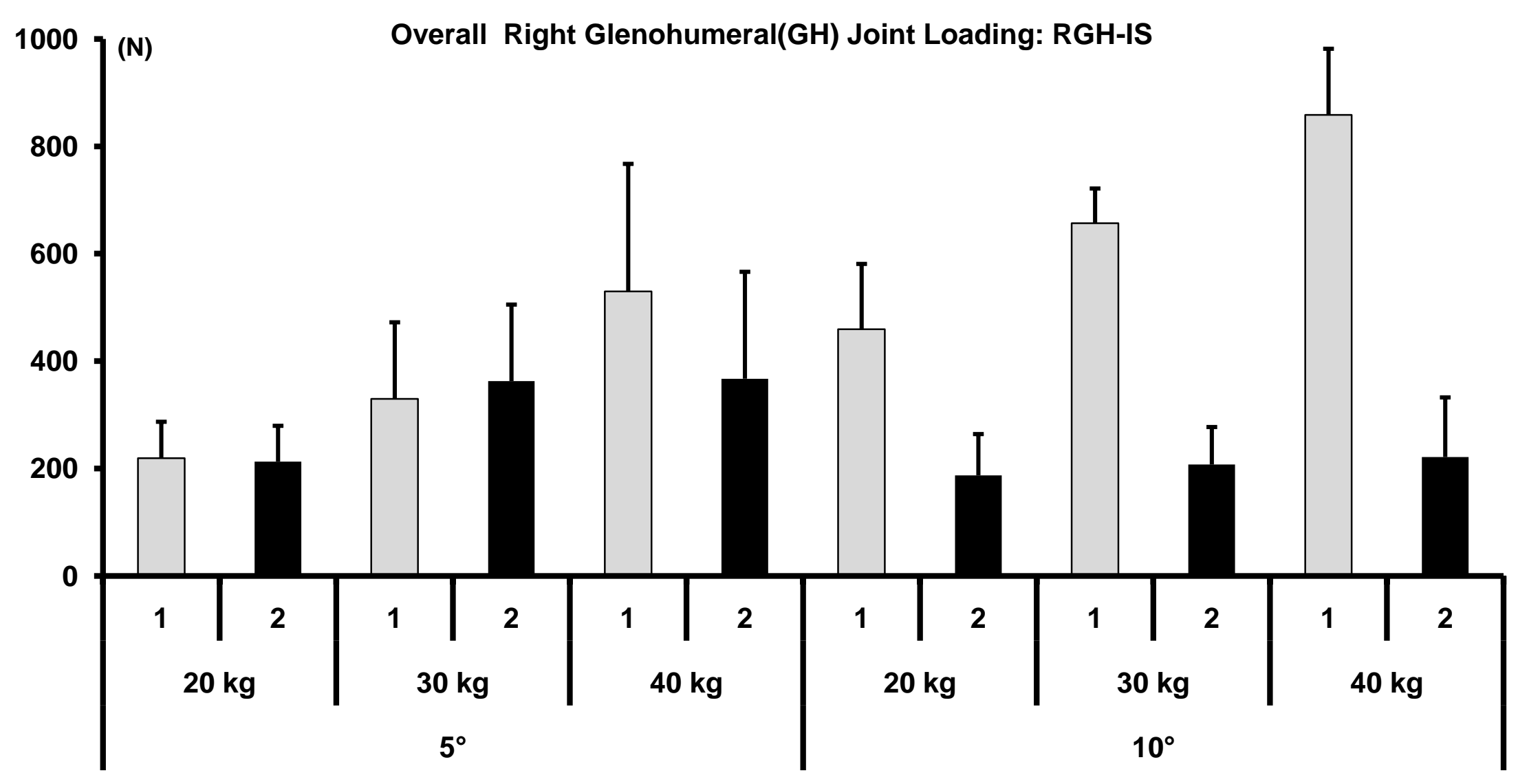

\begin{tabular}{|c|c|c|c|c|c|c|c|c|c|c|c|c|c|c|c|c|c|c|}
\hline \multicolumn{6}{|c|}{$5^{\circ}$} & \multicolumn{6}{|c|}{$10^{\circ}$} & \multirow{2}{*}{\multicolumn{7}{|c|}{$\mathrm{p}$-value }} \\
\hline \multicolumn{2}{|c|}{$20 \mathrm{~kg}$} & \multicolumn{2}{|c|}{$30 \mathrm{~kg}$} & \multicolumn{2}{|c|}{$40 \mathrm{~kg}$} & \multicolumn{2}{|c|}{$20 \mathrm{~kg}$} & \multicolumn{2}{|c|}{$30 \mathrm{~kg}$} & \multicolumn{2}{|c|}{$40 \mathrm{~kg}$} & & & & & & & \\
\hline 1 & 2 & 1 & 2 & 1 & 2 & 1 & 2 & 1 & 2 & 1 & 2 & $\mathrm{~A}^{*}$ & B & $\mathrm{C}$ & $A \times B$ & $\mathrm{~A} \times \mathrm{C}$ & $\mathrm{B} \times \mathrm{C}$ & $\mathrm{A} \times \mathrm{B} \times \mathrm{C}$ \\
\hline $\begin{array}{l}219 \\
(68)\end{array}$ & $\begin{array}{l}213 \\
(67)\end{array}$ & $\begin{array}{c}330 \\
(143)\end{array}$ & $\begin{array}{c}363 \\
(143)\end{array}$ & $\begin{array}{c}530 \\
(238)\end{array}$ & $\begin{array}{c}367 \\
(199)\end{array}$ & $\begin{array}{c}459 \\
(122)\end{array}$ & $\begin{array}{l}187 \\
(77)\end{array}$ & $\begin{array}{l}657 \\
(65)\end{array}$ & $\begin{array}{l}207 \\
(70)\end{array}$ & $\begin{array}{c}858 \\
(123)\end{array}$ & $\begin{array}{c}221 \\
(111)\end{array}$ & $<0.01$ & $<0.01$ & $<0.01$ & 0.88 & $<0.01$ & $<0.01$ & 0.03 \\
\hline
\end{tabular}

$\mathrm{A}=$ walkway gradient; $\mathrm{B}=$ load weight $\mathrm{C}=$ direction of force application

Figure 6.27: Peak force acting at the glenohumeral $(\mathrm{GH})$ joint in the inferior-superior direction during uphill pushing and downhill pulling 


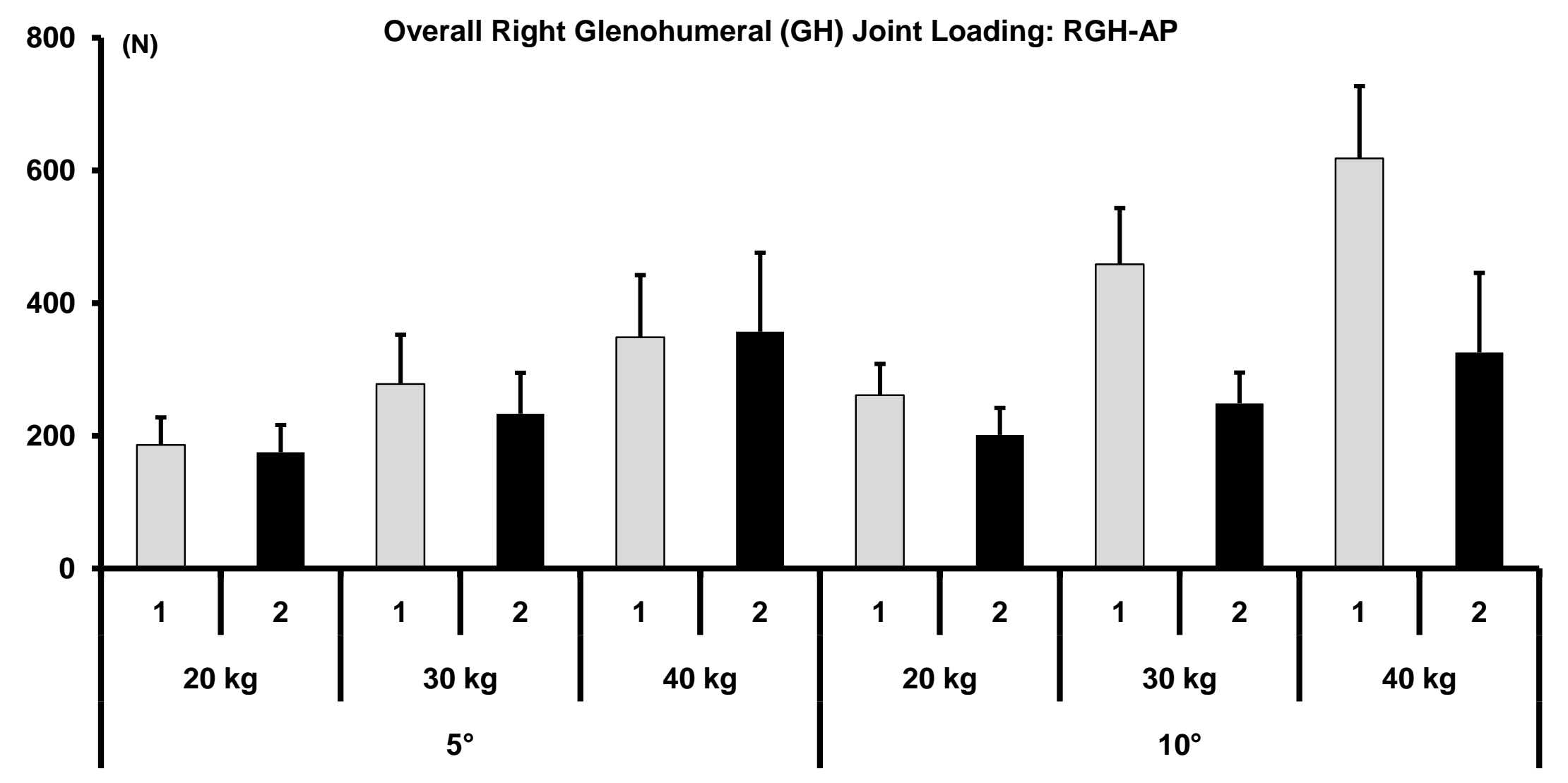

\begin{tabular}{|c|c|c|c|c|c|c|c|c|c|c|c|c|c|c|c|c|c|c|}
\hline \multicolumn{6}{|c|}{$5^{\circ}$} & \multicolumn{6}{|c|}{$10^{\circ}$} & \multirow{2}{*}{\multicolumn{7}{|c|}{ p-value }} \\
\hline \multicolumn{2}{|c|}{$20 \mathrm{~kg}$} & \multicolumn{2}{|c|}{$30 \mathrm{~kg}$} & \multicolumn{2}{|c|}{$40 \mathrm{~kg}$} & \multicolumn{2}{|c|}{$20 \mathrm{~kg}$} & \multicolumn{2}{|c|}{$30 \mathrm{~kg}$} & \multicolumn{2}{|c|}{$40 \mathrm{~kg}$} & & & & & & & \\
\hline 1 & 2 & 1 & 2 & 1 & 2 & 1 & 2 & 1 & 2 & 1 & 2 & $\mathrm{~A}^{*}$ & B & $\mathrm{C}$ & $A \times B$ & $\mathrm{~A} \times \mathrm{C}$ & $\mathrm{B} \times \mathrm{C}$ & $\mathrm{A} \times \mathrm{B} \times \mathrm{C}$ \\
\hline $\begin{array}{l}186 \\
(41)\end{array}$ & $\begin{array}{l}175 \\
(41)\end{array}$ & $\begin{array}{l}278 \\
(75)\end{array}$ & $\begin{array}{l}234 \\
(62)\end{array}$ & $\begin{array}{l}349 \\
(94)\end{array}$ & $\begin{array}{c}357 \\
(119)\end{array}$ & $\begin{array}{l}261 \\
(47)\end{array}$ & $\begin{array}{c}202 \\
(41)\end{array}$ & $\begin{array}{l}458 \\
(85)\end{array}$ & $\begin{array}{l}249 \\
(46)\end{array}$ & $\begin{array}{c}618 \\
(109)\end{array}$ & $\begin{array}{c}326 \\
(120)\end{array}$ & $<0.01$ & $<0.01$ & $<0.01$ & 0.04 & $<0.01$ & $<0.01$ & $<0.01$ \\
\hline
\end{tabular}

$\mathrm{A}=$ walkway gradient; $\mathrm{B}=$ load weight $\mathrm{C}=$ direction of force application

Figure 6.28: Peak force acting at the glenohumeral (GH) joint in the anterior-posterior direction during uphill pushing and downhill pulling 


\section{CHAPTER 7: DISCUSSION}

The current study was designed to quantify the effects of load weight, walkway gradient, and direction of force application on musculoskeletal loading during pushing and pulling a cart on a wooden walkway. The musculoskeletal loading was characterized by the peak joint reaction forces acting at the L5S1 joint of the low back and three shoulder joints (e.g., sternoclavicular, acromioclavicular, and glenohumeral joint) in three anatomical directions.

The discussion of the findings of this study is divided into four major sections. In the first section, the effects of load weight, walkway gradient, and direction of force application on low back loading are discussed. The second section focuses on the effects of the aforementioned factors on shoulder joint loading. In the third section, limitations of the current study are presented and finally, based on the findings of the present study, some practical implications are formulated in the fourth section.

\subsection{Low Back Loading}

\subsubsection{Effect of load weight}

The magnitude of peak shear forces in the lateral direction at the L5S1 joint was very small $(<150 \mathrm{~N})$ compared to the reaction forces in the anterior-posterior and proximal-distal directions. The reaction forces in these two directions increased with the increase in the magnitude of load weight. This observation is in agreement with a number of previous studies. Jäger et al. (2007) and Laursen and Schibye (2002) reported similar effect of load mass on the resultant lumbar-disc compression forces. Hoozemans (2004) also concluded that load weight had a significant effect on the mechanical load at the low back in terms of peak compression forces and net moments.

Although the overall trends observed in the behavior of shear and compression forces at L5S1 joint in this study are similar with the previous studies, the actual magnitudes of the reaction forces found in this study are relatively higher than the previous studies. Laursen and Schibye (2002) reported the peak compression and anterior-posterior shear forces of 751 and $952 \mathrm{~N}$, and 43 and $80 \mathrm{~N}$ at the L4L5 joint for load weights of $25 \mathrm{~kg}$ and $50 \mathrm{~kg}$, respectively. In 
the present study, for the load weights of 30 and $40 \mathrm{~kg}$, the peak compression forces were 863 and $1432 \mathrm{~N}$ and the peak anterior-posterior shear forces were 170 and $331 \mathrm{~N}$, respectively. Another study, Jäger et al. (2007) reported the peak compression forces of $1200 \mathrm{~N}$ and $1500 \mathrm{~N}$ during a cart pushing tasks performed along a $5^{\circ}$ inclined surface for the cart weights (combined weight of cart and load) of 40 and $65 \mathrm{~kg}$, respectively. In the present study the weight of the cart was $14 \mathrm{~kg}$, therefore, for 30 and $40 \mathrm{~kg}$ weight conditions the cart weights (combined weight of cart and load) was approximately 45 and $55 \mathrm{~kg}$, respectively. For these weights, during the pushing tasks performed along a $5^{\circ}$ gradient, the peak compression forces at L5S1 were $1500 \mathrm{~N}$ and $1900 \mathrm{~N}$, respectively. The most likely explanation for the higher low back compression and/or anterior-posterior shear forces observed in this study is the difference in the biomechanical models. In the studies of Jäger et al. (2007) and Laursen and Schibye (2002), low back loading was computed using a single equivalent muscle model. This type of simple biomechanical models employs either one back muscle or one abdominal muscle to compute the joint loading and neglects the coactivation of the other back and trunk muscles. The coactivation of trunk muscles has been found to substantially increase the mechanical loads such as compression and shear forces on the lumbar spine (Hoozemans, et al., 2004; Garg \& Kapellusch, 2009). The present study used a more comprehensive biomechanical model that consider the muscle co-contraction and minimum/maximum muscle recruitment strategy to calculate the joint reaction forces acting at the low back joint. On average, compressive and anterior-posterior shear forces estimated by the model used in this study were $34 \%$ and $75 \%$ higher than the previous studies. These percentage values are to some extent in agreement with the finding of Garg \& Kapellusch (2009), who argued that using a single equivalent muscle model might underestimate spine compression forces by as much as $45 \%$ and shear forces by as much as $70 \%$ (Garg \& Kapellusch, 2009).

\subsubsection{Effect of walkway gradient}

It was found that the peak compression and anterior-posterior shear forces acting at the L5S1 joint increased with the increase of walkway inclination. Only one previous study by Jäger et al. (2007) reported mechanical load on the low back during pushing and pulling along an inclined floor. The results of the present study concerning the effect of walkway gradient are in agreement with the findings of Jäger et al. (2007), who also found that the lumbar load in terms 
of L5S1 compression increased with the increase in the floor gradient. At $0^{\circ}$ and $5^{\circ}$ inclined surfaces, L5S1 peak compression forces of 800 and $900 \mathrm{~N}$ and 1200 and $1500 \mathrm{~N}$ were reported by Jäger et al. (2007) for of the cart weights (combined weight of cart and load) of 45 and $65 \mathrm{~kg}$, respectively. In the present study, for the similar conditions, the peak compression of 1066 and $1432 \mathrm{~N}$, and 1500 and $1900 \mathrm{~N}$, were observed. A higher loading found the present study is mainly due to co-contraction of the trunk muscles as explained in the last paragraph. In a different type of study, Bennett et al. (2008) found that uphill pushing and downhill pulling efforts resulted in higher hand forces compared to level pushing, indicating higher demands on the musculoskeletal system during pushing/pulling exertions performed over inclined surfaces.

\subsubsection{Effect of direction of force application}

One of the main finding of this study was that uphill pushing caused larger spinal forces than downhill pulling activities. The results indicated that the low back joint loading was considerably affected by the direction of force application along the ramps. The largest peak compression force of $3222 \mathrm{~N}$ at the L5S1 joint was observed during uphill pushing on a $10^{\circ}$ ramp for the load weight of $40 \mathrm{~kg}$. This force was $2000 \mathrm{~N}$ more than the corresponding peak compression force during downhill pulling. The largest peak anterior-posterior shear force of $806 \mathrm{~N}$ was found when pushing the $40 \mathrm{~kg}$ weight upward on the $10^{\circ} \mathrm{ramp}$. This force was decreased to $238 \mathrm{~N}$ during downhill pulling under the same weight and gradient conditions. On average, uphill pushing operations imposed up to $26.4 \%$ and $38.3 \%$ greater peak compression and anterior-posterior shear forces compared to downhill pulling. No previous study has reported the effect of direction of force application along the inclined surface on the lumbar load. (Bennett, et al., 2008) reported that peak hand forces during uphill efforts were $31-40 \%$ higher than peak downhill efforts. Jäger et al. (2007) reported that pushing and pulling of the large containers lead to the highest lumbar load in cases of superimposition of considerable trolley mass and considerable floor gradient. This is to some extent in agreement with the current study that uphill pushing of considerable load weight (40 kg weight) along the considerable floor gradient $\left(10^{\circ}\right.$ ramp) constantly generated the higher low back loading than any other combinations of the task factors (load weight, walkway gradient, and direction of force application). However, the effect of direction of force application along the inclined surface on the lumbar load was not reported in the study of Jäger et al. (2007). 
Higher load levels during uphill pushing than the downhill pulling could be explained by two factors. The first explanation is the difference in overall technique used between these two types of exertions. During uphill pushing in order to improve stability and overcome the downward effect of load gravity, the participants exerted more efforts to control the load weight. In this context, the cart handle was kept high and farther away from the trunk and shoulder joints, resulting in a larger lever arm of the joints and higher musculoskeletal loading. Contrarily, during downhill pulling, the cart handle was kept close to trunk and shoulder joints causing a smaller lever arm and thus eliciting lower joint loading. The second explanation for the higher peak forces at the L5S1 joint during uphill pushing was the forward flexed trunk postures used during these exertions. During uphill pushing along the ramp higher trunk inclination required substantial co-activations of the trunk flexors to stabilize the low back joints. Most of these oblique flexor muscles have a large horizontal muscle fiber orientation and limited crosssectional area and therefore generate significant shear and compression forces at the L5S1 joint (Knapik \& Marras, 2009).

\subsubsection{Risk of low back injury}

In the present study, the peak compression forces of $2420 \mathrm{~N}$ and $3222 \mathrm{~N}$ were observed for $30 \mathrm{~kg}$ and $40 \mathrm{~kg}$ weight conditions, respectively, when pushing the cart uphill along the $10^{\circ}$ ramp. These values are below the recommended NIOSH limit of $3400 \mathrm{~N}$ (NIOSH, 1981) but are closer to/higher than the limit of $2500 \mathrm{~N}$, which was chosen as the criterion for evaluating low back disc compression during trolley maneuvers by Jäger et al. (2007). Regarding the shear forces, a tolerance limit of approximately $500 \mathrm{~N}$ was suggested by McGill (2007). The peak shear forces in the lateral direction observed in this study were negligible $(140 \mathrm{~N})$ compared to the recommended criterion. However, the shear forces in the anterior-posterior (AP) direction were found to be higher than $500 \mathrm{~N}$ during uphill pushing along the $10^{\circ}$ ramp at $30 \mathrm{~kg}$ and $40 \mathrm{~kg}$ load weight conditions. Thus, some risk of low back injury, mainly due to increased loading in the anterior-posterior shear direction, is associated with uphill pushing exertions performed along $10^{\circ}$ ramp for the load weight of $30 \mathrm{~kg}$ (combined weight of $44 \mathrm{~kg}$ ) and higher. A similar conclusion was made by Knapik and Marras (2009) for pushing exertions based on the identical trend in the compression and shear forces observed during pushing exertions. 


\subsection{Shoulder Loading}

\subsubsection{Effects of load, gradient, and direction of force application}

In this study, the mechanical load in terms of joint forces acting at the three joints of the shoulder complex was quantified. The variations in walkway gradient as well as the load weight and the direction of force application along the ramp significantly affected the forces acting at the sternoclavicular (SC), acromioclavicular (AC) and glenohumeral (GH) joints.

Similar to the trend found for the low back loading, the main findings of shoulder joint loading are as following: as the load weight increased, so did the resultant peak forces in three directions acting at the shoulder joints; the shoulder loading increased with an increase of gradient; uphill pushing generated larger forces around the shoulder joints than downhill pulling. The findings of effect of load weight on shoulder joint loading are partially in agreement with studies of Schibye et al. (2001) and Laursen and Schibye (2002). Schibye et al. (2001) found that shoulder loading in terms of torques were larger during pushing and pulling of $50 \mathrm{~kg}$ of waste container compared to $25 \mathrm{~kg}$ waste container. Laursen and Schibye (2002) reported that there was a significant increase in shoulder torque magnitude when container weight was increased. The effects of walkway gradient and direction of force application on the mechanical load of shoulder complex joints have not been explored before in the literature.

A few studies have reported mechanical load on the shoulder joint during pushing and pulling, in terms of net moments (De Looze, et al., 2000; Van der Woude, et al., 1995; Abel \& Frank, 1991). However, no previous study have reported the loading of the three shoulder joints, e.g. SC, AC, and GH joints in three anatomical directions during pushing pulling exertions. The study of Hoozemans et al. (2004) is the only available study that reported compression forces in the inferior-superior direction at the GH joint. In this study, the authors have reported that the compression forces at the GH joint were significantly affected by the load weight and the direction of force application. The authors of this study have also presented generalized

estimating equations (GEE) for quantifying GH loading during pushing/pulling exertions. Based on this equation for the load weight of $40 \mathrm{~kg}$ along a horizontal surface, a compression force of $482 \mathrm{~N}$ was estimated at the $\mathrm{GH}$ joint. This value is close to the peak compression force of $455 \mathrm{~N}$ estimated in the present study. 


\subsubsection{Comparison among shoulder complex joints}

The results showed that the largest reaction forces were acted upon at the GH joint, while the forces activated around the SC joint were the smallest. This can be explained by the anatomical structure of these joints in the shoulder complex (see Section 2.3). Compared to the $\mathrm{SC}$ and $\mathrm{AC}$ joints, the $\mathrm{GH}$ joint has the largest range of motion (RoM) and uses more muscle forces for stabilization during the motion. The co-contraction of the muscle around the $\mathrm{GH}$ joint contributes to the higher reaction forces at this joint. Contrastingly, the RoM of the SC joint is rather small and there is hardly any active muscle tissue surrounding this joint to exert forces, and consequently, forces due to muscle co-contraction are negligible (van Drongelen, van der Woude, \& Veeger, 2011). A similar behavior of shoulder joints, i.e. relatively higher loading for the GH joint compared to the other two shoulder joints ( $\mathrm{SC}$ and $\mathrm{AC}$ ) is also reported by van Drongelen et al. (2011). During wheelchair-related propulsion activities, van Drongelen et al. (2011) found that the reaction forces on the SC and AC joints were only one third of those on the GH joint. However, a comparison of the resultant shoulder joint forces between the two studies is not possible because of the totally different nature of the tasks studied. In the context of the current study, the largest shoulder joint forces were observed when pushing the load weight of $40 \mathrm{~kg}$ uphill along a gradient of $10^{\circ}$. The peak forces in three directions (distraction, inferiorsuperior, and anterior-posterior) at the $\mathrm{GH}$ joint were as high as $1357 \mathrm{~N}, 858 \mathrm{~N}$, and $618 \mathrm{~N}$, respectively. The peak forces found at the AC joint were: $910 \mathrm{~N}, 1078 \mathrm{~N}$, and $727 \mathrm{~N}$, respectively, for lateral, inferior-superior, and anterior-posterior shear forces. The corresponding forces at the SC joint were $211 \mathrm{~N}, 200 \mathrm{~N}$, and $61 \mathrm{~N}$, respectively. Although the loading of the SC joint is comparatively lower than the $\mathrm{GH}$ joint, the force acting at the $\mathrm{AC}$ joint are quite high and are comparable with the GH joint. In the existing literature the focus has always been on the loading at the GH joint. The results of the present study show that, in addition to the GH joint, $\mathrm{AC}$ joint is also loaded to higher levels during the uphill pushing exertions. The higher loading of the AC joint observed in this study may explain musculoskeletal problems reported for this joint by van Drongelen et al. (2011) and Buttaci et al. (2004) during pushing exertions. 


\subsubsection{Risk of shoulder injury}

Shoulder tendinitis, rotator cuff pain, and nonspecific shoulder pain are the common work-related shoulder complex pathologies. A general pathomechnism for most of these pain syndromes is the inflammation of muscle tendons caused by their impingement in the bony structures in the shoulder region. The tendons to the rotator cuff muscles and the long head of the biceps brachii are common sites for inflammation in the shoulder. During the uphill pushing activities along a ramp, the shoulder joint was flexed combined with abduction, and arm rotation. Such posture of shoulder demands a relative higher loading for the $\mathrm{AC}$ and $\mathrm{GH}$ joints, mainly in the inferior-superior and distraction directions. The forces acting in these anatomical directions may cause impingement of the rotator cuff muscles (supraspinatus, infraspinatus, teres minor, and subscapularis muscles) (Silverstein, et al., 1998) during such exertion. This can also accelerate the degeneration of these muscles because of the impaired circulation caused by the impingement. As a consequence, the uphill pushing activities along an inclined walkway may impose a detrimental impact in the shoulder region.

Little is known about the safe loading limits for the shoulder joints. A tolerance limit of $500 \mathrm{~N}$, which is same as the tolerance limit for the low back shear forces is used in this study to evaluate the risk of shoulder injuries during pushing/pulling exertions. In the following situations the loading of the shoulder joints exceeded $500 \mathrm{~N}$ :

(1) For the AC joint in the lateral and AP directions: while pushing the load weight of 30 $\mathrm{kg}$ and $40 \mathrm{~kg}$ uphill along a walkway gradient of $10^{\circ}$; (four situations)

(2) For the GH joint in distraction (lateral) direction: while pushing the load weight of 30 $\mathrm{kg}$ and $40 \mathrm{~kg}$ uphill along the two gradients $\left(5^{\circ}\right.$ and $\left.10^{\circ}\right)$ of the walkway. (four situations)

When compared with L5S1 joint, only in two of the above eight situations, a risk of low back injury due to higher anterior-posterior shear loading was identified. Clearly, shoulder joint is found to be at a relatively higher risk of injuries during the uphill pushing activities than the low back. However, this claim was based on a tolerance limit of 500 N. Future studies evaluating accurate tolerance limits for the shoulder joints are needed to more accurately understand the risk of injuries during occupational exertions. 


\subsection{Study Limitations}

There are a few limitations of this study that need to be acknowledged. First, the findings of this study are a function of the experimental conditions investigated. It should be noted that during uphill pushing hand forces were directed away from the body, while during downhill pulling the hand forces were directed toward the body. In both cases, the participants walked forward along a ramped wooden walkway. At workplaces the workers operate on different types of surfaces, which may be different from the wooden surface used in this study. These surfaces may provide different levels of resistance against the movement of the cart and may affect the directions and magnitudes of the pushing /pulling forces and the overall technique and working postures differently. Moreover, the cart used in this study is one of the most frequently used carts at workplaces. Other carts with different dimensions are also used at various other workplaces. Different cart dimensions, especially handle height and physical dimensions of the cart, with respect to its center of mass could affect direction of the forces as well as the posture and movement, and therefore, the mechanical load on the musculoskeletal system (Hoozemans, et al., 2004).

Second, only sustained pushing and pulling phases were investigated, while no attention was paid to the initial phase of accelerating objects and the ending phase of decelerating the object and bringing it to a standstill (Bennett, et al., 2008; van Dieen \& Kingma, 1999). These motion phases could result in different musculoskeletal loading. In a study performed by Bennett et al. (2008), it was found that during uphill pushing, initial phase of accelerating objects was the most taxing, whereas during downhill pulling, ending phase was found to impose the greatest stress on the musculoskeletal system.

Third, the effects of load weight, walkway gradient, and direction of force application along ramps are specific to the population used in the current study. Participation was voluntary and the participant pool was limited to university-aged students with little to no manual materials handling experience.

\subsection{Practical Implication}

In order to prevent work-related MSD, it is important to know the exact quantitative load acting on the musculoskeletal system during occupational exertions. This information is 
necessary for performing potential risk assessment and for developing appropriate interventions strategies. Although the results of this study demonstrate that low back and shoulder joints loading during pushing and pulling exertions is affected by a number of task factors and their interaction with each other, problematic loading was observed only at a few instances. For the low back, anterior-posterior (AP) shear loads at L5S1 joint was found to be higher than the accepted safe limit. Among the shoulder joints, the highest level of loading was observed for the $\mathrm{AC}$ joint in the inferior-superior (IS) direction and for the GH joint in the distraction (DIS) direction. Among the various experimental conditions evaluated in this study, pushing tasks performed at a walkway gradient of $10^{\circ}$ at 30 and $40 \mathrm{~kg}$ weight conditions were found to be detrimental for the aforementioned joints.

Based on the findings of this study the following regression equations are developed for quantifying loading of the key low back and shoulder joints during uphill pushing exertions (Appendix L). These equations can be used to quantify the effect of tasks factors such as walkway gradient and load weight on the loading of the key low back and shoulder joints during uphill pushing exertions. By comparing the quantitative loading with the safe acceptable loading limits risk for the low back and shoulder MSD injuries during cart pushing tasks performed on inclined surfaces could be identified.

$$
\begin{aligned}
& \text { L5S1-AP }=57.6-15.2 \text { Degree + 5.45 Weight + 1.58 Degree*Weight } \\
& \text { RAC-IS }=-59.9+1.3 \text { Degree + 14.3 Weight + 1.19 Degree*Weight } \\
& \text { RGH-DIS }=10+4.4 \text { Degree + 19.8 Weight + 1.06 Degree*Weight }
\end{aligned}
$$

Where,

Degree = walkway gradient (in degrees),

Weight $=$ load weight transferred by the cart $(\mathrm{kg})$.

It should be noted that these equations were developed using three walkway gradients $\left(0^{\circ}, 5^{\circ}\right.$ and $\left.10^{\circ}\right)$ and three load conditions $(20,30$, and $40 \mathrm{~kg})$. A linear relationship was assumed between the joint loading, and walkway gradient and load weight. 


\section{CHAPTER 8: CONCLUSIONS}

\subsection{Conclusions}

The main conclusions of this study are as follows:

(1) In addition to the load weight, effect of walkway gradient and direction of force application on the loading of low back and shoulder joints during cart pushing and pulling exertions were studied to fill the knowledge gaps in the existing literature. It was found that in addition to the load weight, walkway gradient and direction of force application had a significant effect on the loading of L5S1, sternoclavicular, acromioclavicular and glenohumeral joints. Uphill pushing exertions were found to be more strenuous than the downhill pulling exertions.

(2) The compression forces acting at the L5S1 joint at extreme task conditions $\left(10^{\circ} \mathrm{ramp}, 40 \mathrm{~kg}\right.$ load weight) were found to be lower than the recommended safe loading limit. However, loading of the L5S1 joint in the anterior-posterior (AP) direction was found to exceed the recommended safe loading limit during uphill pushing exertions performed along a $10^{\circ} \mathrm{ramp}$ at 30 and $40 \mathrm{~kg}$ weight conditions. This further suggests that, anterior-posterior shear loading at the L5S1 joint should be considered when assessing the risk of low back musculoskeletal disorders due to pushing and pulling activities.

(3) In this study, for the first time, quantitative loading of the three shoulder complex joints, sternoclavicular, acromioclavicular and glenohumeral joint, in three anatomical directions during cart pushing and pulling exertions were reported. Among the three joints, higher levels of loading were observed for the acromioclavicular and glenohumeral joints. In the existing literature, the focus has always been on the glenohumeral joint and loading of the acromioclavicular joints was not evaluated. The results of this research show that the loading of the acromioclavicular joint was comparable with the glenohumeral joint, further suggesting that the loading of the acromioclavicular joint should be considered, in addition to the glenohumeral joint, while evaluating risk of shoulder musculoskeletal disorders due to pushing and pulling activities. 
- Acromioclavicular and glenohumeral joints were loaded to higher levels of forces $(>500 \mathrm{~N}$ ) at a number of instances during pushing/pulling exertions evaluated in this study. Accurate estimate in terms of risk of injuries cannot be made because of the lack of information on the safe loading limits of these joints. However, based on the peak forces acting at these joints in the three anatomical directions, it can be concluded that during uphill pushing exertions, shoulder joint injury may occur due to the higher shear forces in the distraction (DIS) direction for the glenohumeral joint and higher compression (inferior-superior) forces for the acromioclavicular joint.

\subsection{Future Work}

The results of this study were obtained based on dynamic pushing/pulling activities performed on the wooden walkway using only one type of cart. Further studies should investigate the effect of different types of surfaces and carts on the musculoskeletal loading during pushing and pulling activities. Furthermore, there is a need to investigate a wider range of gradients since a strong interaction effect between gradient and direction of force application on the musculoskeletal loading was observed in this study. Future study with a wider range of gradients may assist in identifying a more accurate relationship between musculoskeletal loading and pushing/pulling exertions performed over inclined surfaces, further allowing development of a more accurate mathematical equation that could be used for the assessment of risk of low back and shoulder MSD.

Future study should also look at a larger, more diverse working population with manual materials handling experience to determine how well the trends observed in the current study could be applied to the working population.

One of the main contributions of this study is the quantitative assessment of the loading of three shoulder joints during dynamic cart pushing/pulling tasks. In fact this is the first study that reports this type of data. Based on the findings of this study it was identified that acromioclavicular and glenohumeral joints were loaded to higher levels of forces (> $500 \mathrm{~N})$ at various instances. Because of the lack of data on the tolerance limits of these joints, accurate 
assessment of the risk of shoulder joint injuries could not be presented. There is a strong need for the future studies that could provide safe loading limits for these joints. 


\section{BIBLIOGRAPHY}

[1] National Research Council and Institute of Medicine. (2001). Musculoskeletal disorders and the workplace: Low back and upper extremities. Washington, DC: National Academy Press.

[2] Dunning, KK, Davis, KG, Cook, C, et al. (2010). Costs by industry and diagnosis among musculoskeletal claims in a state workers compensation system: 1999-2004. American Journal of Industrial Medicine, 53, 276-284.

[3] Bureau of Labor Statistics. (2010). Nonfatal Occupational Injuries and Illnesses Requiring Days Away From Work, 2009. Retrieved April 12, 2011, from http://www.bls.gov/news.release/osh2.nr0.htm

[4] Nonnenmann, M, Anton, D, Gerr, F, et al. (2008). Musculoskeletal symptoms of the neck and upper extremities among Iowa dairy farmers. American Journal of Industrial Medicine, 51(6), 443-451.

[5] Hess, J, Kincl, L, Amasay, T, \& Wolfe, P. (2010). Ergonomic evaluation of masons laying concrete masonry units and autoclaved aerated concrete. Applied Ergonomics, 41(3), 477-483.

[6] Holmström, E, Lindell, J, \& Moritz, U. (1992). Low back and neck/shoulder pain in construction workers: Occupational workload and psychosocial risk factors.Part 2: relationship to neck and shoulder pain. Spine, 17(6), 672-677.

[7] Grooten, W, Wiktorin, C, Norrman, L, et al. (2004). Seeking care for neck/shoulder pain: A prospective study of work-related risk factors in a healthy population. Journal of occupational and environmental medicine, 46(2), 138-146.

[8] Cassou, B, Derriennic, F, Monfort, C, et al. (2002). Chronic neck and shoulder pain, age, and working conditions: Longitudinal results from a large random sample in France. Occupational and Environmental Medicine, 59(8), 537-544.

[9] Snook, S. (1978). The design of manual handling tasks. Ergonomics, 21(12), 963-985.

[10] Smith, J, Woldstad, J, \& Patterson, P. (2009). Ergonomics of manual materials handling. In M Kutz (Ed.), Environmentally Conscious Materials Handling. Hoboken, NJ: John Wiley \& Sons, Inc. 
[11] Marras, W, Cutlip, RG, Burt, SE, \& Waters, TR. (2009). National occupational research agenda (NORA) future directions in occupational musculoskeletal disorder health research. Applied Ergonomics, 40(1), 15-22.

[12] Lee, P, \& Granata, K. (2006). Interface stability influences torso muscle recruitment and spinal load during pushing tasks. Ergonomics, 49(3), 235-248.

[13] Waters, T, Nelson, A, \& Proctor, C. (2007). Patient handling tasks with high risk for musculoskeletal disorders in critical care. Critical Care Nursing Clinics of North America, 19(2), 131-143.

[14] Seto, E, Roach, C, Chagpar, A, \& MacDonald, A. (2006). Usability evaluations as part of the procurement process: Case study of hospital point of care carts. Paper presented at the Proceedings of the Human Factors and Ergonomics Society 50th Annual Meeting, Santa Monica, CA.

[15] Das, B, \& Wimpee, J. (2002). Ergonomics evaluation and redesign of a hospital meal cart. Applied Ergonomics, 33(4), 309-318.

[16] Gledhill, N, \& Jamnik, V. (1992). Characterization of the physical demands of firefighting. Canadian Journal of Sport Sciences, 17(3), 207-213.

[17] Glitsch, U, Ottersbach, H, Ellegast, R, et al. (2007). Physical workload of flight attendants when pushing and pulling trolleys aboard aircraft. International Journal of Industrial Ergonomics, 37(11-12), 845-854.

[18] Schaub, K, Berg, K, Winter, G, et al. (2007). Muscular capabilities and workload of flight attendants for pushing and pulling trolleys aboard aircraft. International Journal of Industrial Ergonomics, 37(11-12), 883-892.

[19] Jäger, M, Sawatzki, K, Glitsch, U, et al. (2007). Load on the lumbar spine of flight attendants during pushing and pulling trolleys aboard aircraft. International Journal of Industrial Ergonomics, 37(11-12), 863-876.

[20] Hoozemans, M, Slaghuis, W, Faber, G, \& van Dieën, J. (2007). Cart pushing: The effects of magnitude and direction of the exerted push force, and of trunk inclination on low back loading. International Journal of Industrial Ergonomics, 37(11-12), 832-844.

[21] Hoozemans, M, Kuijer, P, Kingma, I, et al. (2004). Mechanical loading of the low back and shoulders during pushing and pulling activities. Ergonomics, 47(1), 1-18. 

differences in exerted forces and physiological load during pushing and pulling of wheeled cages by postal workers. Ergonomics, 43(2), 269-281.

[23] Schibye, B, Søgaard, K, Martinsen, D, \& Klausen, K. (2001). Mechanical load on the low back and shoulders during pushing and pulling of two-wheeled waste containers compared with lifting and carrying of bags and bins. Clinical Biomechanics, 16(7), 549559.

[24] Frings-Dresen, M, Kemper, H, Stassen, A, et al. (1995). Guidelines for energetic load in three methods of refuse collecting. Ergonomics, 38(10), 2056-2064.

[25] Frings-Dresen, M, Kemper, H, Stassen, A, et al. (1995). The daily work load of refuse collectors working with three different collecting methods: a field study. Ergonomics, 38(10), 2045-2055.

[26] Søgaard, K, Laursen, B, Jensen, B, \& Sjøgaard, G. (2001). Dynamic loads on the upper extremities during two different floor cleaning methods. Clinical Biomechanics, 16(10), 866-879.

[27] Kumar, S. (1995). Electromyography of spinal and abdominal muscles during garden raking with two rakes and rake handles. Ergonomics, 38(9), 1793-1804.

[28] Kumar, S, Narayan, Y, \& Bacchus, C. (1995). Symmetric and asymmetric twohanded pull-push strength of young adults. Human Factors, 37(4), 854-865.

[29] Chaffin, D. (1987). Manual materials handling and the biomechanical basis for prevention of low back pain in industry-- an overview. American Industrial Hygiene Association Journal 48, 989 - 996.

[30] Hoozemans, M, van der Beek, A, Frings-Dresen, M, et al. (1998). Pushing and pulling in relation to musculoskeletal disorders: a review of risk factors. Ergonomics, 41(6), 757-781.

[31] van der Beek, A, Frings-Dresen, M, van Dijk, F, et al. (1993). Loading and unloading by lorry drivers and musculoskeletal complaints. International Journal of Industrial Ergonomics, 12(1-2), 13-23.

[32] Ozguler, A, Leclerc, A, Landre, MF, et al. (2000). Individual and occupational determinants of low back pain according to various definitions of low back pain. Journal of epidemiology and community health, 54(3), 215-220. 
[33] Klein, BP, Jensen, RC, \& Sanderson, LM. (1984). Assessment of workers' compensation claims for back strains/sprains. Journal of Occupational and Environmental Medicine, 26(6), 443-448.

[34] Harber, P, Billet, E, Lew, M, \& Horan, M. (1987). Importance of non-patient transfer activities in nursing-related back pain: I. Questionnaire survey. Journal of Occupational and Environmental Medicine, 29(12), 967-970.

[35] Garg, A, \& Moore, J. (1992). Epidemiology of low-back pain in industry. Occupational Medicine, 7, 593-608.

[36] Fuortes, LJ, Shi, Y, Zhang, M, et al. (1994). Epidemiology of back injury in university hospital nurses from review of workers' compensation records and a casecontrol survey. Journal of Occupational and Environmental Medicine, 36(9), 1022-1026.

[37] Frymoyer, JW, Pope, MH, Costanza, MC, et al. (1980). Epidemiologic studies of low-back pain. Spine, 5(5), 419-423.

[38] Damkot, D, Pope, M, Lord, J, \& Frymoyer, J. (1984). The relationship between work history, work environment and low-back pain in men. Spine, 9(4), 395-399.

[39] Smedley, J, Inskip, H, Trevelyan, F, et al. (2003). Risk factors for incident neck and shoulder pain in hospital nurses. Occupational and environmental medicine, 60(11), 864.

[40] Hoozemans, M, van der Beek, A, Frings-Dresen, M, et al. (2002a). Low-back and shoulder complaints among workers with pushing and pulling tasks. Scandinavian Journal of Work, Environment \& Health, 28(5), 293-303.

[41] Hoozemans, M, Van der Beek, A, Frings-Dresen, M, et al. (2002b). Pushing and pulling in association with low back and shoulder complaints. Occupational and environmental medicine, 59(10), 696-702.

[42] Chaffin, D, Andres, R, \& Garg, A. (1983). Volitional postures during maximum push/pull exertions in the sagittal plane. Human Factors, 25(5), 541-550.

[43] Kroemer, K. (1974). Horizontal push and pull forces exertable when standing in working positions on various surfaces. Applied Ergonomics, 5, 94-102.

[44] Ayoub, M, \& Mcdaniel, J. (1974). Effects of operator stance on pushing and pulling tasks. American Institute of Industrial Engineering Transactions, 6, 185-195. 

on pushing and pulling. Paper presented at the 4th International Cyberspace Conference on Ergonomics, Johannesburg.

[46] Resnick, M, \& Chaffin, D. (1995). An ergonomic evaluation of handle height and load in maximal and submaximal cart pushing. Applied Ergonomics, 26(3), 173-178.

[47] Bennett, A, Desai, S, Todd, A, \& Freeland, H. (2008). The effects of load and gradient on hand force responses during dynamic pushing and pulling tasks. Ergonomics SA: Journal of the Ergonomics Society of South Africa, 20(1), 3-15.

[48] van der Beek, A, \& Frings-Dresen, M. (1995). Physical workload of lorry drivers: a comparison of four methods of transport. Ergonomics, 38(7), 1508-1520.

[49] Laursen, B, \& Schibye, B. (2002). The effect of different surfaces on biomechanical loading of shoulder and lumbar spine during pushing and pulling of twowheeled containers. Applied Ergonomics, 33(2), 167-174.

[50] Allread, W, Marras, W, \& Burr, D. (2000). Measuring trunk motions in industry: variability due to task factors, individual differences, and the amount of data collected. Ergonomics, 43(6), 691-701.

[51] Marras, W, Lavender, S, Leurgans, S, et al. (1993). The role of dynamic threedimensional trunk motion in occupationally-related low back disorders: the effects of workplace factors, trunk position, and trunk motion characteristics on risk of injury. Spine, 18(5), 617-628.

[52] Mital, A, Nicholson, A, \& Ayoub, M. (1997). A guide to manual materials handling. London: Taylor and Francis.

[53] van der Beek, A, Hoozemans, M, Frings-Dresen, M, \& Burdorf, A. (1999). Assessment of exposure to pushing and pulling in epidemiological field studies: an overview of methods, exposure measures, and measurement strategies. International Journal of Industrial Ergonomics, 24(4), 417-429.

[54] Garg, A, Funke, S, \& Janisch, D. (1988). One-handed dynamic pulling strength with special application to lawn mowers. Ergonomics, 31(8), 1139-1153.

[55] Grieve, D. (1983). Slipping due to manual exertion. Ergonomics, 26(1), 61-72. 
NIOSH. (1997). Musculoskeletal disorders and workplace factors: a critical review of epidemiologic evidence for work-related musculoskeletal disorders of the neck, upper extremity, and low back. Cincinnati, $\mathrm{OH}$ : NIOSH.

NIOSH. (1981). Work Practices Guide for Manual Lifting: Tech. Report No. 81122, U.S. Dept. of Health and Human Services (NIOSH), Cincinnati, OH.

[58] National Institute for Occupational Safety and Health. (1997). Musculoskeletal disorders and workplace factors: a critical review of epidemiologic evidence for workrelated musculoskeletal disorders of the neck, upper extremity, and low back. Cincinnati, OH: NIOSH.

[59] Hughes, RE, Silverstein, BA, \& Evanoff, BA. (1997). Risk factors for work related musculoskeletal disorders in an aluminum smelter. American journal of industrial medicine, 32(1), 66-75.

[60] Reese, NB, Bandy, WD, \& Yates, C. (2009). Joint range of motion and muscle length testing.: Saunders.

[61] Culham, E, \& Peat, M. (1993). Functional anatomy of the shoulder complex. Journal of Orthopaedic and Sports Physical Therapy, 18, 342-342.

[62] Garcin, M, Cravic, J, Vandewalle, H, \& Monod, H. (1996). Physiological strains while pushing or hauling. European Journal of Applied Physiology and Occupational Physiology, 72(5), 478-482.

[63] Lee, K, Chaffin, D, Herrin, G, \& Waikar, A. (1991). Effect of handle height on lower-back loading in cart pushing and pulling. Applied Ergonomics, 22(2), 117-123.

[64] Lee, K, Chaffin, D, Walker, A, \& Chung, M. (1989). Lower back muscle forces in pushing and pulling. Ergonomics 32 (12), 1551-1563.

[65] Mital, A, Kopardekar, P, \& Motorwala, A. (1995). Isokinetic pull strengths in the vertical plane: effects of speed and arm angle. Clinical Biomechanics, 10(2), 110-112.

[66] Jung, M-C, Haight, JM, \& Freivalds, A. (2005). Pushing and pulling carts and two-wheeled hand trucks. International Journal of Industrial Ergonomics, 35(1), 79-89.

[67] Mack, K, Haslegrave, C, \& Gray, M. (1995). Usability of manual handling aids for transporting materials. Applied Ergonomics, 26(5), 353-364. 
[68] St-Vincent, M, Denis, D, Imbeau, D, \& Laberge, M. (2005). Work factors affecting manual materials handling in a warehouse superstore. International Journal of Industrial Ergonomic, 35, 33-46.

[69] Kingma, I, Kuijer, P, Hoozemans, M, et al. (2003). Effect of design of twowheeled containers on mechanical loading. International Journal of industrial Ergonomics, 31, 73-86.

[70] Waters, T, Putz-Anderson, V, Garg, A, \& Fine, L. (1993). Revised NIOSH equation for the design and evaluation of manual lifting tasks. Ergonomics, 36(7), 749776.

[71] Norman, R, \& McGill, S. (1993). WATBAK (version 5.1) - User's Manual. In University of Waterloo (Ed.). Waterloo, Canada.

[72] Lay, AN, Hass, CJ, \& Gregor, RJ. (2006). The effects of sloped surfaces on locomotion: a kinematic and kinetic analysis. Journal of Biomechanics, 39(9), 16211628.

[73] Li, KW, Chang, WR, Lin, CH, \& Wei, JC. (2006). Relationship between the measured friction coefficients of floors on a horizontal surface and on a $10^{\circ} \mathrm{ramp}$. International Journal of Industrial Ergonomics, 36(8), 705-711.

[74] Li, KW, Chang, WR, Wei, JC, \& Kou, CH. (2006). Friction measurements on ramps using the Brungraber Mark II slipmeter. Safety science, 44(5), 375-386.

[75] Prentice, SD, Hasler, EN, Groves, JJ, \& Frank, JS. (2004). Locomotor adaptations for changes in the slope of the walking surface. Gait \& posture, 20(3), 255-265.

[76] McIntosh, AS, Beatty, KT, Dwan, LN, \& Vickers, DR. (2006). Gait dynamics on an inclined walkway. Journal of Biomechanics, 39(13), 2491-2502.

[77] Cham, R, \& Redfern, MS. (2004). Gait adaptations during load carrying on level and inclined surfaces. Occupational Ergonomics, 4(1), 11-26.

[78] S. Redfern, M, \& DiPasquale, J. (1997). Biomechanics of descending ramps. Gait \& posture, 6(2), 119-125.

[79] DiPasquale, J. (1997). Biomechanics of descending ramps. Gait and Posture, 6(2), 119-125.

[80] Redfern, MS, Cham, R, Gielo-Perczak, K, et al. (2001). Biomechanics of slips. Ergonomics, 44(13), 1138-1166. 
[81] Cham, R, \& Redfern, MS. (2002a). Heel contact dynamics during slip events on level and inclined surfaces. Safety science, 40(7-8), 559-576.

[82] Cham, R, \& Redfern, MS. (2002b). Changes in gait when anticipating slippery floors. Gait \& posture, 15(2), 159-171.

[83] De Looze, M, Van Greuningen, K, Rebel, J, et al. (2000). Force direction and physical load in dynamic pushing and pulling. Ergonomics, 43(3), 377-390.

[84] Li, K, Chang, C, \& Chang, W. (2008). Slipping of the foot on the floor when pulling a pallet truck. Applied Ergonomics, 39(6), 812-819.

[85] De Looze, M, Stassen, A, Markslag, A, et al. (1995). Mechanical loading on the low back in three methods of refuse collecting. Ergonomics, 38(10), 1993-2006.

[86] Schibye, B, Søgaard, K, Laursen, B, \& Sjøgaard, G. (1997). Mechanical load on the spine during pushing and pulling. Paper presented at the Proceedings of the 13th Triennial Congress of the International Ergonomics Association, Finland.

[87] Schibye, B, Sogaard, K, Martinsen, D, \& Klausen, K. (2001). Mechanical load on the low back and shoulders during pushing and pulling of two-wheeled waste containers compared with lifting and carrying of bags and bins. Clinical Biomechanics (Bristol), 16, 549-559.

[88] Kumar, S. (1990). Cumulative load as a risk factor for back pain. Spine, 15, 13111316.

[89] van Dieen, JH, \& de Looze, MP. (1999). Sensitivity of single-equivalent trunk extensor muscle models to anatomical and functional assumptions. Journal of biomechanics, 32(2), 195-198.

[90] University of Michigan. (2011). 3D Static Strength Prediction Program ${ }^{\text {TM }}$ Version 6.0.5. Retrieved May 25, 2011, from http://www.engin.umich.edu/dept/ioe/3DSSPP/example.html

[91] Knapik, G, \& Marras, W. (2009). Spine loading at different lumbar levels during pushing and pulling. Ergonomics, 52(1), 60-70.

[92] Andres, R, \& Chaffin, D. (1991). Validation of a biodynamic model of pushing and pulling. Journal of biomechanics, 24(11), 1033-1045.

[93] Garg, A, \& Kapellusch, J. (2009). Applications of biomechanics for prevention of work-related musculoskeletal disorders. Ergonomics, 52(1), 36-59. 
[94] Granata, K, \& Marras, W. (1995a). The influence of trunk muscle coactivity on dynamic spinal loads. Spine, 20(8), 913.

[95] Marras, W, \& Granata, K. (1997a). The development of an EMG-assisted model to assess spine loading during whole-body free-dynamic lifting. Journal of Electromyography and Kinesiology, 7(4), 259-268.

[96] Marras, W, \& Granata, K. (1995). A biomechanical assessment and model of axial twisting in the thoracolumbar spine. Spine, 20(13), 1440.

[97] Granata, K, \& Marras, W. (1995b). An EMG-assisted model of trunk loading during free-dynamic lifting. Journal of Biomechanics, 28(11), 1309-1317.

[98] Marras, W, \& Sommerich, C. (1991a). A three-dimensional motion model of loads on the lumbar spine: II. Model validation. Human Factors: The Journal of the Human Factors and Ergonomics Society, 33(2), 139-149.

[99] Marras, W, \& Sommerich, C. (1991b). A three-dimensional motion model of loads on the lumbar spine: I. Model structure. Human Factors: The Journal of the Human Factors and Ergonomics Society, 33(2), 123-137.

[100] Granata, K, \& Marras, W. (1993). An EMG-assisted model of loads on the lumbar spine during asymmetric trunk extensions. Journal of Biomechanics, 26(12), 1429-1438.

[101] Marras, WS, \& Granata, KP. (1997b). The development of an EMG-assisted model to assess spine loading during whole-body free-dynamic lifting. Journal of Electromyography and Kinesiology, 7(4), 259-268.

[102] van Dieen, JH. (1997). Are recruitment patterns of the trunk musculature compatible with a synergy based on the maximization of endurance? Journal of Biomechanics, 30(11-12), 1095-1100.

[103] van Dieen, JH, \& Kingma, I. (1999). Total trunk muscle force and spinal compression are lower in asymmetric moments as compared to pure extension moments. Journal of Biomechanics, 32(7), 681-687.

[104] Keyserling, WM. (2000). Workplace risk factors and occupational musculoskeletal disorders, Part 2: A review of biomechanical and psychophysical research on risk factors associated with upper extremity disorders. AIHAJ-American Industrial Hygiene Association, 61(2), 231-243. 
[105] Kuijer, P, van der Beek, A, van Dieën, J, et al. (2002). Effect of the number of two-wheeled containers at a gathering point on the energetic workload and work efficiency in refuse collecting. [doi: DOI: 10.1016/S0003-6870(02)00040-6]. Applied Ergonomics, 33(6), 571-577.

[106] Lett, KK, \& McGill, SM. (2006). Pushing and pulling: personal mechanics influence spine loads. Ergonomics, 49(9), 895-908.

[107] Kingma, I, \& van Dieen, JH. (2004). Lifting over an obstacle: effects of onehanded lifting and hand support on trunk kinematics and low back loading. Journal of Biomechanics, 37(2), 249-255.

[108] Keyserling, WM, Monroe, KA, Woolley, CB, \& Ulin, SS. (1999). Ergonomic considerations in trucking delivery operations: An evaluation of hand trucks and ramps. American Industrial Hygiene Association Journal, 60(1), 22-31.

[109] Fischer, H, Kirchberg, S, \& Moessner, T. (2009). Biomechanical Gait Analysis for the Extraction of Slip Resistance Test Parameters. Industrial Health, 47(6), 617-625.

[110] You, JY, Chou, YL, Lin, CJ, \& Su, FC. (2001). Effect of slip on movement of body center of mass relative to base of support. Clinical Biomechanics, 16(2), 167-173.

[111] Janz, KF, Rao, S, Baumann, HJ, \& Schultz, JL. (2003). Measuring children's vertical ground reaction forces with accelerometry during walking, running, and jumping: The Iowa bone development study. Pediatric Exercise Science, 15(1), 34-43.

[112] Desailly, E, Daniel, Y, Sardain, P, \& Lacouture, P. (2009). Foot contact event detection using kinematic data in cerebral palsy children and normal adults gait. Gait \& posture, 29(1), 76-80.

[113] Andreoni, G, Mazzola, M, Ciani, O, et al. (2009). Method for Movement and Gesture Assessment (MMGA) in Ergonomics. Digital Human Modeling, 591-598.

[114] Hwang, S, \& Kim, Y. (2009). Lower extremity joint kinetics and lumbar curvature during squat and stoop lifting. BMC Musculoskeletal Disorders, 10(1), 15-24.

[115] Riley, PO, Paolini, G, Della Croce, U, et al. (2007). A kinematic and kinetic comparison of overground and treadmill walking in healthy subjects. Gait \& posture, 26(1), 17-24. 
[116] Kojima, S, Nakajima, Y, \& Takada, J. (2008). Kinematics of the Compensatory Step by the Trailing Leg Following an Unexpected Forward Slip while Walking. Journal of physiological anthropology, 27(6), 309-315.

[117] Rasmussen, J, Damsgaard, M, \& Voigt, M. (2001). Muscle recruitment by the $\min /$ max criterion - a comparative numerical study. Journal of Biomechanics, 34(3), 409-415.

[118] Rasmussen, J, Damsgaard, M, \& Christensen, ST. (2001, July 8-13, 2001). Optimization of human motion: to invert inverse dynamics. Paper presented at the International Society of Biomechanics, XVIIIth Congress, Zurich, Switzerland.

[119] Wagner, D, Rasmussen, J, \& Reed, M. (2007). Assessing the importance of motion dynamics for ergonomic analysis of manual materials handling tasks using the AnyBody Modeling System. Paper presented at the 2007 Digital Human Modeling Conference, Seattle, WA, USA.

[120] Dubowsky, S, Rasmussen, J, Sisto, S, \& Langrana, N. (2008). Validation of a musculoskeletal model of wheelchair propulsion and its application to minimizing shoulder joint forces. Journal of Biomechanics, 41(14), 2981-2988.

[121] Wu, J, An, K, Cutlip, R, et al. (2008). Analysis of musculoskeletal loading in an index finger during tapping. Journal of Biomechanics, 41(3), 668-676.

[122] Wu, J, Chiou, S, \& Pan, C. (2009). Analysis of Musculoskeletal Loadings in Lower Limbs During Stilts Walking in Occupational Activity. Annals of biomedical engineering, 37(6), 1177-1189.

[123] Vicon. Plug-in Gait Manual. Retrieved July 18, 2011, from www.ircweb.co.jp/vicon_web/news_bn/PIGManualver1.pdf

[124] Erdemir, A, McLean, S, Herzog, W, \& van den Bogert, AJ. (2007). Model-based estimation of muscle forces exerted during movements. Clinical Biomechanics, 22(2), 131-154.

[125] Morrow, M, Kaufman, KR, \& An, KN. (2010). Shoulder model validation and joint contact forces during wheelchair activities. Journal of Biomechanics, 43(13), 24872492. 
[126] Marras, WS, \& Sommerich, CM. (1991c). A three-dimensional motion model of loads on the lumbar spine: II. Model validation. Human Factors: The Journal of the Human Factors and Ergonomics Society, 33(2), 139-149.

[127] Plamondon, A, Gagnon, M, \& Desjardins, P. (1996). Validation of two 3-D segment models to calculate the net reaction forces and moments at the joint in lifting. Clinical Biomechanics, 11(2), 101-110.

[128] De Looze, M, Kingma, I, Bussmann, J, \& Toussaint, H. (1992). Validation of a dynamic linked segment model to calculate joint moments in lifting. Clinical Biomechanics, 7(3), 161-169.

[129] Fong, DTP, Chan, YY, Hong, Y, et al. (2008). A three-pressure-sensor (3PS) system for monitoring ankle supination torque during sport motions. Journal of Biomechanics, 41(11), 2562-2566.

[130] Wong, WY, \& Wong, MS. (2008). Trunk posture monitoring with inertial sensors. European Spine Journal, 17(5), 743-753.

[131] Plamondon, A, Delisle, A, Larue, C, et al. (2007). Evaluation of a hybrid system for three-dimensional measurement of trunk posture in motion. Applied Ergonomics, 38(6), 697-712.

[132] McGill, S. (2007). Low back disorders: evidence-based prevention and rehabilitation. Champaign, IL: Human Kinetics Publishers.

[133] Van der Woude, L, Van Konlngsbruggen, C, Kroes, A, \& Kingma, I. (1995). Effect of push handle height on net moments and forces on the musculoskeletal system during standardized wheelchair pushing tasks. Prosthetics and Orthotics International, 19(3), 188-201.

[134] Abel, E, \& Frank, T. (1991). The design of attendant propelled wheelchairs. Prosthetics and Orthotics International, 15(1), 38.

[135] van Drongelen, S, van der Woude, L, \& Veeger, H. (2011). Load on the shoulder complex during wheelchair propulsion and weight relief lifting. Clinical Biomechanics, 26(5), 452-457.

[136] Buttaci, CJ, Stitik, TP, Yonclas, PP, \& Foye, PM. (2004). Osteoarthritis of the acromioclavicular joint: a review of anatomy, biomechanics, diagnosis, and treatment. American journal of physical medicine \& rehabilitation, 83(10), 791. 
[137] Silverstein, B, Welp, E, Nelson, N, \& Kalat, J. (1998). Claims incidence of workrelated disorders of the upper extremities: Washington state, 1987 through 1995. American journal of public health, 88(12), 1827. 
APPENDIX A: PHYSICAL ACTIVITY READINESS QUESTIONNAIRE 


\section{Physical Activity Readiness Questionnaire (PAR-Q)}

For most people physical activity should not pose any problem or hazard. PAR-Q has been designed to identify the small number of adults for whom physical activity might be inappropriate or those who should have medical advice concerning the type of activity most suitable for them.

YES NO

\begin{tabular}{|c|c|c|}
\hline$\square$ & $\square$ & $\begin{array}{l}\text { 1. Has your doctor ever said you have a heart trouble and should only do physical } \\
\text { activity recommended by a doctor? }\end{array}$ \\
\hline 口 & $\square$ & 2. Do you frequently suffer from chest pain? \\
\hline & $\square$ & 3. Do you often faint or have spells of severe dizziness? \\
\hline & $\square$ & 4. Has your doctor ever said your blood pressure was too high? \\
\hline & $\square$ & $\begin{array}{l}\text { 5. Has your doctor ever told you that you have a bone or joint problem such as } \\
\text { arthritis that has been aggravated by, or might be made worse with exercise. }\end{array}$ \\
\hline & $\square$ & $\begin{array}{l}\text { 6. Is there any good physical reason why you should not follow an activity } \\
\text { program even if you want to? }\end{array}$ \\
\hline & 口 & 7. Are you 65 and not accustomed to vigorous exercise? \\
\hline
\end{tabular}

If you answer "yes" to any question, vigorous exercise or exercise testing should be postponed. Medical clearance may be necessary.

I have read this questionnaire, I understand it does not provide medical assessment in lieu of a physical examination by a physician.

Participant's signature:

Date:

Investigator's signature:

Date:

Adapted from PAR-Q validation report, British Columbia department of Health, June 1975.

Reference: BQ Hafen, WWK Hoeger (1994), Wellness: Guidelines for a healthy lifestyle. Englewood, CO: Morton Pub. Co. 
APPENDIX B: CONSENT AND INFORMATION FORM 


\section{WestVirginiaUniversity. Office of Research Compliance CONSENT AND INFORMATION FORM}

\section{OMR ICF}

Principal Investigator: Nimbarte, Ashish

Department: ENGINEERING - Ind./Mgt. Sys. Engineering

Tracking Number: H-22994

Study Title: Effects of Load and Gradient on Musculoskeletal Loading during Dynamic Twowheeled Cart Pushing and Pulling

\section{Co-Investigator(s):}

Sun, Yun

\section{Sponsor}

\section{Contact Persons}

In the event you experience any side effects or injury related to this research, you should contact Dr.Nimbarte at 304/293-9473. (After hours contact Dr.Nimbarte at 225/226-8813.)

If you have any questions, concerns, or complaints about this research, you can contact Dr. Nimbarte at 304/293-9473 or Yun Sun at 304/293-9669.

If you have any questions, concerns, or complaints about this research that are not answered by the research team, you can contact the IRB Office at 304/293-7073. You can also call this number if you cannot talk to the research team or want to talk to someone else.

\section{Introduction}

You, , have been asked to participate in this research study, which has been explained to you by Dr. Ashish Nimbarte, Ph.D., and Yun Sun, M.S.. This study is being conducted by Dr. Ashish Nimbarte, Ph.D. and Yun Sun, M.S. in the Department of Industrial and

Tracking \#: Approved On:

Valid Through:

Last Amended:
Page 1 of 5

$$
\text { Initials }
$$

Date 
Management Systems Engineering (IMSE) at West Virginia University. This research is being conducted to fulfill the requirements for a doctoral dissertation of Ms. Yun Sun in the area of Occupational Safety and Health in the Department of IMSE at West Virginia University, under the supervision of Dr. Nimbarte.

\section{Purposes of the Study}

The purpose of this study is to learn more about biomechanical loading of major body joints and segments during dynamic cart pushing and pulling tasks under varying task demands. WVU expects to enroll approximately twenty male subjects.

\section{Description of Procedures}

Using a two-wheeled hand cart, the experiment will be conducted in three different loading states $(20,30$ and $40 \mathrm{~kg})$ on three different gradients $\left(0^{\circ}, 5^{\circ}\right.$ and $\left.10^{\circ}\right)$ of the wooden walkway during pushing and pulling. The study will take approximately 2 hours to complete from the time you arrived in the lab. Each trial consists of three successive pushing or pulling maneuvers over a distance of about $5 \mathrm{~m}$ of wooden walkway, which ensured a sufficient coefficient of friction to avoid slipping. Between these maneuvers, the participant will be allowed to rest for about $5 \mathrm{~s}$. The entire measurement period for a trial amounts to a maximum of $45 \mathrm{~s}$. Between the trials of varying gradients, there will be a recovery break lasing at least $10 \mathrm{~min}$, during which the test setup for a ramped pathway are modified. Tasks were designed to ensure that participants would not exceed accepted levels of energy expenditure. You may stop participating at any time, and for any reason.

\section{Risks and Discomforts}

It is possible that participants may experience some soreness in their right shoulder and arm shortly after completing the study due to the repeated pulling of the loaded cart on a ramped walkway. With the sufficient rest periods designed between each set of pulling tasks, the risk of injury as a result of participating in this study is minimal. Furthermore, at any time during the study, if you feel uncomfortable with any method or performing the requirements, you will be allowed to withdraw from the study without any penalty. In case of muscle or ligament strain (chances of which are very slim) you will be referred to Family Medicine Center at West Virginia University.

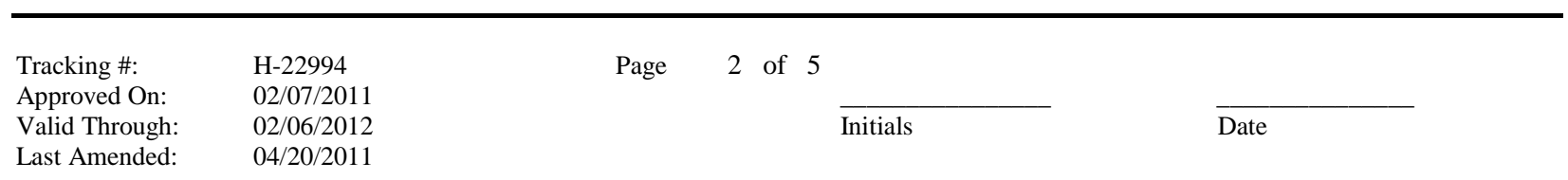




\section{Alternatives}

You do not have to participate in this study.

\section{Benefits}

You may not receive any direct benefit from this study. The successful completion of this study will allow us to establish a new method to quantify the loading of the musculoskeletal system during a variety of cart push and pull exertions. It is expected that, this new knowledge will allow us to more precisely identify, the physical risk factors associated with work-related musculoskeletal disorders. The findings of this research are expected to have a positive impact on the health of the worker in various occupational groups because, by using the results of this study, workplace methods and/or designs could be appropriately modified to reduce the incidence of excessive musculoskeletal loading, further preventing the development of musculoskeletal disorders.

\section{Financial Considerations}

No monetary compensation will be given for participating in this study and participants do not incur any costs as a result of participation in the study.

\section{Voluntary Compensation}

It is very important for you to understand that neither the investigator nor WVU or it associated affiliates has the funds set aside to pay for the cost of lost work wages or any care or treatment that might be necessary because you get hurt or sick taking part in this study. Any injuries that may result from this study would not be eligible for Workers' Compensation as this is not jobrelated injury. Understand that any treatments necessary will be billed to the participant or to your personal health insurance, and you may wish to consult your insurance provider before participating in this study.

\begin{tabular}{llllll}
\hline & & Page 3 of 5 & & \\
Tracking \#: & H-22994 & & & & Initials \\
Approved On: & $02 / 07 / 2011$ & & & & Date \\
Valid Through: & $02 / 06 / 2012$ & & & &
\end{tabular}




\section{Confidentiality}

Any information about you that is obtained as a result of your participation in this research will be kept as confidential as legally possible. Your research records and test results, just like hospital records, may be subpoenaed by court order or may be inspected by federal regulatory authorities without your additional consent. In any publications that result from this research, neither your name nor any information from which you might be identified will be published without your consent.

\section{Voluntary Participation}

Participation in this study is voluntary. You are free to withdraw your consent to participate in this study at any time. Refusal to participate or withdrawal will not affect your future care, [or your employee status at West Virginia University or your class standing or grades, as appropriate] and will involve no penalty to you. In the event new information becomes available that may affect your willingness to participate in this study, this information will be given to you so that you can make an informed decision about whether or not to continue your participation. You have been given the opportunity to ask questions about the research, and you have received answers concerning areas you did not understand.

Page

4 of 5

$$
\text { Initials }
$$

Date 
Upon signing this form, you will receive a copy.

I willingly consent to participate in this research.

Signature of Subject or

Printed Name

Date

Time

Subjects Legal Representative

The participant has had the opportunity to have questions addressed. The participant willingly agrees to be in the study.

Tracking \#: Approved On:

Valid Through:

Last Amended:
$\mathrm{H}-22994$ $02 / 07 / 2011$ $02 / 06 / 2012$ $04 / 20 / 201$
Page 5 of 5

Initials

Date 
APPENDIX C: SAMPLE SIZE CALCULATIONS 
Table C-1: Sample size calculations for Model 1

\begin{tabular}{|c|c|c|c|c|c|c|c|c|c|c|c|c|c|c|c|c|c|c|c|c|c|c|c|c|}
\hline $\begin{array}{c}\text { Dependent } \\
\text { Variables }\end{array}$ & c & $\lambda$ & $v_{1}$ & $v_{2}$ & $\boldsymbol{\beta}$ & $\begin{array}{c}\text { Power } \\
(1-\beta)\end{array}$ & $\mathbf{c}_{1}$ & $\lambda$ & $v_{1}$ & $v_{2}$ & $\beta$ & $\begin{array}{c}\text { Power } \\
(1-\beta)\end{array}$ & $\mathbf{c}_{2}$ & $\lambda$ & $v_{1}$ & $v_{2}$ & $\beta$ & $\begin{array}{c}\text { Power } \\
(1-\beta)\end{array}$ & $\mathbf{c}_{3}$ & $\lambda$ & $v_{1}$ & $v_{2}$ & $\boldsymbol{\beta}$ & $\begin{array}{c}\text { Power } \\
(1-\beta)\end{array}$ \\
\hline L5S1-ML & 5 & 5.40 & 4 & 8 & 0.00 & 1.00 & & & & & & & & & & & & & & & & & & \\
\hline L5S1-PD & 5 & 4.48 & 4 & 8 & 0.07 & 0.93 & & & & & & & & & & & & & & & & & & \\
\hline L5S1-AP & 5 & 3.59 & 4 & 8 & 0.14 & 0.86 & 6 & 3.91 & 5 & 10 & 0.05 & 0.95 & & & & & & & & & & & & \\
\hline RSC-ML & 5 & 2.05 & 4 & 8 & 0.50 & 0.50 & 6 & 2.20 & 5 & 10 & 0.34 & 0.66 & 7 & 2.34 & 6 & 12 & 0.20 & 0.80 & 8 & 2.48 & 7 & 14 & 0.10 & 0.90 \\
\hline RSC-IS & 5 & 2.56 & 4 & 8 & 0.38 & 0.62 & 6 & 2.77 & 5 & 10 & 0.16 & 0.84 & 7 & 2.96 & 6 & 12 & 0.08 & 0.92 & & & & & & \\
\hline RSC-AP & 5 & 2.51 & 4 & 8 & 0.40 & 0.60 & 6 & 2.72 & 5 & 10 & 0.21 & 0.79 & 7 & 2.90 & 6 & 12 & 0.08 & 0.92 & & & & & & \\
\hline RAC-ML & 5 & 4.19 & 4 & 8 & 0.08 & 0.92 & & & & & & & & & & & & & & & & & & \\
\hline RAC-IS & 5 & 4.30 & 4 & 8 & 0.08 & 0.92 & & & & & & & & & & & & & & & & & & \\
\hline RAC-AP & 5 & 4.69 & 4 & 8 & 0.06 & 0.95 & & & & & & & & & & & & & & & & & & \\
\hline RGH-DIS & 5 & 4.65 & 4 & 8 & 0.06 & 0.95 & & & & & & & & & & & & & & & & & & \\
\hline RGH-IS & 5 & 4.56 & 4 & 8 & 0.06 & 0.94 & & & & & & & & & & & & & & & & & & \\
\hline RGH-AP & 5 & 6.99 & 4 & 8 & 0.00 & 1.00 & & & & & & & & & & & & & & & & & & \\
\hline
\end{tabular}

Table C-2: Sample size calculations for Model 2

\begin{tabular}{|c|c|c|c|c|c|c|c|c|c|c|c|c|c|c|c|c|c|c|c|c|c|c|c|c|}
\hline $\begin{array}{c}\text { Dependent } \\
\text { Variables }\end{array}$ & c & $\lambda$ & $v_{1}$ & $v_{2}$ & $\beta$ & $\begin{array}{c}\text { Power } \\
(1-\beta)\end{array}$ & $\mathbf{c}_{1}$ & $\lambda$ & $v_{1}$ & $v_{2}$ & $\boldsymbol{\beta}$ & $\begin{array}{c}\text { Power } \\
(1-\beta)\end{array}$ & $\mathbf{c}_{2}$ & $\lambda$ & $v_{1}$ & $v_{2}$ & $\beta$ & $\begin{array}{c}\text { Power } \\
(1-\beta)\end{array}$ & $\mathbf{c}_{3}$ & $\lambda$ & $v_{1}$ & $v_{2}$ & $\beta$ & $\begin{array}{c}\text { Power } \\
(1-\beta)\end{array}$ \\
\hline L5S1-ML & 5 & 3.79 & 4 & 44 & 0.06 & 0.95 & & & & & & & & & & & & & & & & & & \\
\hline L5S1-PD & 5 & 2.69 & 4 & 44 & 0.19 & 0.81 & 6 & 2.91 & 5 & 55 & 0.08 & 0.92 & & & & & & & & & & & & \\
\hline L5S1-AP & 5 & 3.33 & 4 & 44 & 0.08 & 0.93 & & & & & & & & & & & & & & & & & & \\
\hline RSC-ML & 5 & 6.67 & 4 & 44 & 0.00 & 1.00 & & & & & & & & & & & & & & & & & & \\
\hline RSC-IS & 5 & 3.51 & 4 & 44 & 0.07 & 0.93 & & & & & & & & & & & & & & & & & & \\
\hline RSC-AP & 5 & 2.17 & 4 & 44 & 0.37 & 0.63 & 6 & 2.33 & 5 & 55 & 0.17 & 0.83 & 7 & 2.49 & 6 & 66 & 0.07 & 0.93 & & & & & & \\
\hline RAC-ML & 5 & 1.76 & 4 & 44 & 0.57 & 0.43 & 6 & 1.87 & 5 & 55 & 0.40 & 0.60 & 7 & 1.98 & 6 & 66 & 0.19 & 0.81 & 8 & 2.08 & 7 & 77 & 0.10 & 0.90 \\
\hline RAC-IS & 5 & 3.08 & 4 & 44 & 0.13 & 0.87 & 6 & 3.34 & 5 & 55 & 0.05 & 0.95 & & & & & & & & & & & & \\
\hline RAC-AP & 5 & 2.25 & 4 & 44 & 0.30 & 0.70 & 6 & 2.43 & 5 & 55 & 0.22 & 0.78 & 7 & 2.59 & 6 & 66 & 0.05 & 0.95 & & & & & & \\
\hline RGH-DIS & 5 & 3.08 & 4 & 44 & 0.13 & 0.87 & 6 & 3.34 & 5 & 55 & 0.05 & 0.95 & & & & & & & & & & & & \\
\hline RGH-IS & 5 & 5.62 & 4 & 44 & 0.00 & 1.00 & & & & & & & & & & & & & & & & & & \\
\hline RGH-AP & 5 & 3.82 & 4 & 44 & 0.05 & 0.95 & & & & & & & & & & & & & & & & & & \\
\hline
\end{tabular}


APPENDIX D: ANOVA TABLES FOR MODEL VALIDATION 


\section{ANOVA of Session 1: RMSE, $r$ versus Subject, Weight}

Analysis of Variance for RMSE, using Adjusted SS for Tests

Source DF Seq SS Adj SS Adj MS F P

$\begin{array}{lllllll}\text { Subject } \quad 5 & 44.862 & 44.862 & 8.972 & 3.12 & 0.059\end{array}$

$\begin{array}{lllllll}\text { Weight } & 2 & 19.683 & 19.683 & 9.841 & 3.42 & 0.074\end{array}$

$\begin{array}{lllll}\text { Error } & 10 & 28.759 & 28.759 & 2.876\end{array}$

Total $17 \quad 93.303$

$\mathrm{S}=1.69585 \quad \mathrm{R}-\mathrm{Sq}=69.18 \% \quad \mathrm{R}-\mathrm{Sq}(\mathrm{adj})=47.60 \%$

Analysis of Variance for $r$, using Adjusted SS for Tests

Source DF Seq SS Adj SS Adj MS F P

$\begin{array}{lllllll}\text { Subject } & 5 & 0.0089829 & 0.0089829 & 0.0017966 & 3.38 & 0.048\end{array}$

$\begin{array}{lllllll}\text { Weight } \quad 2 & 0.0006601 & 0.0006601 & 0.0003301 & 0.62 & 0.557\end{array}$

$\begin{array}{lllll}\text { Error } & 10 & 0.0053232 & 0.0053232 & 0.0005323\end{array}$

Total $17 \quad 0.0149663$

$\mathrm{S}=0.0230721 \quad \mathrm{R}-\mathrm{Sq}=64.43 \% \quad \mathrm{R}-\mathrm{Sq}(\mathrm{adj})=39.53 \%$

ANOVA of Session 2: RMSE, $r$ versus Subject, Degree, Weight, Direction

Analysis of Variance for RMSE, using Adjusted SS for Tests

$\begin{array}{lrrrrrr}\text { Source } & \text { DF } & \text { Seq SS } & \text { Adj SS } & \text { Adj MS } & \text { F } & P \\ \text { Subject } & 5 & 1163.395 & 1163.395 & 232.679 & 49.40 & 0.000 \\ \text { Degree } & 1 & 5.752 & 5.752 & 5.752 & 1.22 & 0.274 \\ \text { Weight } & 2 & 55.581 & 55.581 & 27.790 & 5.90 & 0.005 \\ \text { Direction } & 1 & 35.182 & 35.182 & 35.182 & 7.47 & 0.008 \\ \text { Degree*Weight } & 2 & 5.317 & 5.317 & 2.658 & 0.56 & 0.572 \\ \text { Degree*Direction } & 1 & 28.969 & 28.969 & 28.969 & 6.15 & 0.016 \\ \text { Weight*Direction } & 2 & 1.768 & 1.768 & 0.884 & 0.19 & 0.829 \\ \text { Degree*Weight*Direction } & 2 & 4.223 & 4.223 & 2.111 & 0.45 & 0.641 \\ \text { Error } & 55 & 259.045 & 259.045 & 4.710 & & \\ \text { Total } & 71 & 1559.230 & & & & \\ \text { S }=2.17023 \quad \mathrm{R}-\text { Sq }=83.39 \% & \mathrm{R}-\text { Sq (adj) } & =78.55 \% & & & \end{array}$

Analysis of Variance for $r$, using Adjusted SS for Tests

\begin{tabular}{lrrrrrr} 
Source & DF & Seq SS & Adj SS & Adj MS & $F$ & $P$ \\
Subject & 5 & 0.038139 & 0.038139 & 0.007628 & 5.92 & 0.000 \\
Degree & 1 & 0.017703 & 0.017703 & 0.017703 & 13.74 & 0.000 \\
Weight & 2 & 0.001320 & 0.001320 & 0.000660 & 0.51 & 0.602 \\
Direction & 1 & 0.001522 & 0.001522 & 0.001522 & 1.18 & 0.282 \\
Degree*Weight & 2 & 0.001770 & 0.001770 & 0.000885 & 0.69 & 0.508 \\
Degree*Direction & 1 & 0.000416 & 0.000416 & 0.000416 & 0.32 & 0.572 \\
Weight*Direction & 2 & 0.001361 & 0.001361 & 0.000680 & 0.53 & 0.593 \\
Degree*Weight*Direction & 2 & 0.000858 & 0.000858 & 0.000429 & 0.33 & 0.718 \\
Error & 55 & 0.070885 & 0.070885 & 0.001289 & & \\
Total & 71 & 0.133973 & & & \\
S =0.0359001 R-Sq $=47.09 \%$ & $R-S q(\operatorname{adj})=31.70 \%$ & & \\
\hline
\end{tabular}

Figure D-1: ANOVA Tables for model validation 
APPENDIX E: PARTICIPANTS' ANTHROPOMETRIC DATA 
Table E-1: Participants' anthropometric data

\begin{tabular}{|c|c|c|c|c|c|c|c|c|c|c|c|c|c|}
\hline Subject No. & 1 & 2 & 3 & 4 & 5 & 6 & 7 & 8 & 9 & 10 & 11 & 12 & All \\
\hline $\begin{array}{c}\text { Age } \\
\text { (years) }\end{array}$ & 26 & 28 & 24 & 24 & 23 & 22 & 21 & 22 & 23 & 24 & 25 & 23 & $\begin{array}{l}23.75 \\
(1.91)\end{array}$ \\
\hline $\begin{array}{c}\text { Body mass } \\
(\mathrm{kg})\end{array}$ & 65.5 & 77 & 63.6 & 75 & 59.3 & 70.5 & 72 & 73 & 73.5 & 68.5 & 66.5 & 66.3 & $\begin{array}{l}69.23 \\
(5.19) \\
\end{array}$ \\
\hline $\begin{array}{c}\text { Height } \\
(\mathrm{cm})\end{array}$ & 169 & 165 & 173 & 169.5 & 176 & 171 & 177 & 180 & 171 & 167 & 176 & 170 & $\begin{array}{l}172.04 \\
(4.44) \\
\end{array}$ \\
\hline $\begin{array}{c}\text { Head height } \\
(\mathrm{cm})\end{array}$ & 18.5 & 15.4 & 16.3 & 18.4 & 18.1 & 16.5 & 18.4 & 16.8 & 18 & 18 & 17.3 & 17.2 & $\begin{array}{l}17.41 \\
(0.99) \\
\end{array}$ \\
\hline $\begin{array}{l}\text { Upper arm } \\
\text { length }(\mathrm{cm})\end{array}$ & 32.5 & 32.5 & 37 & 34 & 32.5 & 35 & 35.2 & 36.6 & 35.5 & 34.5 & 35.5 & 31.8 & $\begin{array}{l}34.38 \\
(1.73) \\
\end{array}$ \\
\hline $\begin{array}{l}\text { Lower arm } \\
\text { length }(\mathrm{cm})\end{array}$ & 27.2 & 24.3 & 27.1 & 28.4 & 30 & 28.5 & 27 & 30.1 & 26.7 & 25.8 & 29.1 & 23.9 & $\begin{array}{l}27.34 \\
(2.01)\end{array}$ \\
\hline $\begin{array}{l}\text { Hand length } \\
\text { (cm) }\end{array}$ & 18.5 & 17.5 & 18.4 & 17.3 & 18.1 & 18.8 & 17.7 & 20.5 & 18.3 & 17.8 & 18.4 & 17.7 & $\begin{array}{l}18.25 \\
(0.84)\end{array}$ \\
\hline $\begin{array}{c}\text { Thigh length } \\
\text { (cm) }\end{array}$ & 43 & 39 & 38.16 & 41.7 & 45.3 & 44.7 & 42.5 & 40.4 & 40.7 & 43.8 & 48.4 & 43.9 & $\begin{array}{l}42.63 \\
(2.87) \\
\end{array}$ \\
\hline $\begin{array}{c}\text { Shank length } \\
(\mathrm{cm})\end{array}$ & 43.1 & 37.5 & 41.03 & 41.5 & 44 & 43.5 & 42 & 43.4 & 42.4 & 38.1 & 41 & 37.8 & $\begin{array}{l}41.28 \\
(2.31) \\
\end{array}$ \\
\hline $\begin{array}{l}\text { Foot length } \\
\text { (cm) }\end{array}$ & 26.7 & 27.5 & 26.1 & 26.6 & 28 & 26.1 & 25 & 30.5 & 26.6 & 26 & 26.7 & 26 & $\begin{array}{l}26.82 \\
(1.39)\end{array}$ \\
\hline
\end{tabular}


APPENDIX F: RAW GRF DATA OF RIGHT AND LEFT FOOT 
Table F-1: Raw GRF data for right and left foot in Session 1

\begin{tabular}{|c|c|c|c|c|c|c|c|c|c|c|c|c|c|}
\hline \multirow{2}{*}{$\begin{array}{c}\text { Subject } \\
\text { no. }\end{array}$} & \multirow{2}{*}{ Weight } & \multicolumn{6}{|c|}{ Right Foot } & \multicolumn{6}{|c|}{ Left Foot } \\
\hline & & $\mathbf{F x}$ & Fy & $\mathbf{F z}$ & $\mathbf{M x}$ & My & $\mathbf{M z}$ & $\mathbf{F x}$ & $\mathbf{F y}$ & $\mathbf{F z}$ & $\mathbf{M x}$ & My & Mz \\
\hline 1 & 20 & 65 & 53 & 655 & 79 & 22 & 5 & 70 & 82 & 640 & 36 & 27 & 8 \\
\hline 1 & 30 & 62 & 60 & 654 & 54 & 33 & 2 & 56 & 93 & 650 & 57 & 24 & 8 \\
\hline 1 & 40 & 65 & 77 & 650 & 41 & 38 & 3 & 64 & 107 & 673 & 74 & 6 & 10 \\
\hline 2 & 20 & 56 & 63 & 745 & 81 & 59 & 10 & 58 & 48 & 729 & 31 & 52 & 7 \\
\hline 2 & 30 & 58 & 78 & 735 & 72 & 46 & 10 & 54 & 58 & 720 & 45 & 48 & 6 \\
\hline 2 & 40 & 63 & 88 & 724 & 116 & 35 & 9 & 51 & 73 & 714 & 104 & 52 & 10 \\
\hline 3 & 20 & 50 & 58 & 616 & 65 & 31 & 5 & 58 & 36 & 580 & 45 & 27 & 5 \\
\hline 3 & 30 & 51 & 65 & 610 & 65 & 30 & 5 & 50 & 40 & 605 & 35 & 35 & 4 \\
\hline 3 & 40 & 41 & 68 & 605 & 80 & 42 & 2 & 39 & 42 & 607 & 40 & 40 & 6 \\
\hline 4 & 20 & 59 & 56 & 745 & 99 & 48 & 9 & 60 & 40 & 716 & 33 & 35 & 4 \\
\hline 4 & 30 & 48 & 69 & 739 & 69 & 41 & 7 & 59 & 44 & 712 & 30 & 41 & 4 \\
\hline 4 & 40 & 63 & 87 & 729 & 84 & 47 & 9 & 57 & 50 & 724 & 50 & 43 & 5 \\
\hline 5 & 20 & 51 & 66 & 611 & 68 & 27 & 6 & 54 & 38 & 588 & 33 & 30 & 5 \\
\hline 5 & 30 & 52 & 69 & 606 & 81 & 26 & 4 & 58 & 44 & 587 & 59 & 31 & 6 \\
\hline 5 & 40 & 41 & 78 & 600 & 80 & 44 & 2 & 41 & 52 & 585 & 35 & 40 & 7 \\
\hline 6 & 20 & 39 & 24 & 679 & 41 & 59 & 3 & 43 & 39 & 677 & 41 & 43 & 3 \\
\hline 6 & 30 & 49 & 50 & 670 & 99 & 52 & 8 & 46 & 40 & 673 & 48 & 46 & 5 \\
\hline 6 & 40 & 46 & 68 & 667 & 60 & 56 & 4 & 40 & 56 & 671 & 94 & 43 & 3 \\
\hline 7 & 20 & 76 & 69 & 721 & 101 & 41 & 13 & 73 & 55 & 728 & 36 & 32 & 2 \\
\hline 7 & 30 & 65 & 71 & 711 & 83 & 40 & 10 & 67 & 65 & 696 & 62 & 41 & 2 \\
\hline 7 & 40 & 67 & 87 & 707 & 71 & 25 & 10 & 69 & 66 & 680 & 51 & 36 & 3 \\
\hline 8 & 20 & 60 & 85 & 731 & 117 & 48 & 7 & 70 & 65 & 727 & 39 & 28 & 7 \\
\hline 8 & 30 & 58 & 100 & 724 & 60 & 38 & 3 & 73 & 72 & 717 & 95 & 15 & 7 \\
\hline 8 & 40 & 60 & 119 & 717 & 86 & 44 & 4 & 73 & 97 & 710 & 27 & 25 & 5 \\
\hline 9 & 20 & 55 & 60 & 737 & 78 & 56 & 7 & 55 & 45 & 757 & 28 & 49 & 4 \\
\hline 9 & 30 & 56 & 65 & 722 & 69 & 43 & 7 & 51 & 55 & 726 & 42 & 45 & 3 \\
\hline 9 & 40 & 61 & 77 & 712 & 113 & 32 & 6 & 48 & 80 & 708 & 101 & 49 & 7 \\
\hline 10 & 20 & 55 & 77 & 687 & 36 & 47 & 4 & 49 & 70 & 674 & 55 & 34 & 6 \\
\hline 10 & 30 & 39 & 78 & 680 & 39 & 42 & 5 & 55 & 110 & 669 & 48 & 31 & 7 \\
\hline 10 & 40 & 62 & 106 & 668 & 40 & 50 & 5 & 56 & 133 & 653 & 82 & 24 & 6 \\
\hline
\end{tabular}




\begin{tabular}{|c|c|c|c|c|c|c|c|c|c|c|c|c|c|}
\hline \multirow{2}{*}{$\begin{array}{c}\text { Subject } \\
\text { no. }\end{array}$} & \multirow{2}{*}{ Weight } & \multicolumn{6}{|c|}{ Right Foot } & \multicolumn{6}{|c|}{ Left Foot } \\
\hline & & $\mathbf{F x}$ & Fy & $\mathbf{F z}$ & $\mathbf{M x}$ & My & Mz & $\mathbf{F x}$ & $\mathbf{F y}$ & $\mathbf{F z}$ & Mx & My & $\mathbf{M z}$ \\
\hline 11 & 20 & 41 & 25 & 650 & 42 & 57 & 4 & 44 & 40 & 656 & 42 & 44 & 6 \\
\hline 11 & 30 & 51 & 50 & 662 & 97 & 56 & 5 & 47 & 55 & 670 & 49 & 45 & 5 \\
\hline 11 & 40 & 46 & 64 & 667 & 61 & 57 & 6 & 45 & 56 & 678 & 87 & 45 & 7 \\
\hline 12 & 20 & 75 & 65 & 665 & 84 & 27 & 10 & 75 & 87 & 651 & 41 & 24 & 6 \\
\hline 12 & 30 & 71 & 77 & 662 & 59 & 38 & 7 & 61 & 98 & 645 & 62 & 21 & 4 \\
\hline 12 & 40 & 67 & 88 & 655 & 46 & 43 & 5 & 69 & 110 & 668 & 79 & 3 & 4 \\
\hline
\end{tabular}

Table F-2: Raw GRF data for right and left foot in Session 2

\begin{tabular}{|c|c|c|c|c|c|c|c|c|c|c|c|c|c|c|c|}
\hline \multirow{2}{*}{$\begin{array}{c}\text { Subject } \\
\text { no. }\end{array}$} & \multirow{2}{*}{ Degree } & \multirow{2}{*}{ Weight } & \multirow{2}{*}{ Direction } & \multicolumn{6}{|c|}{ Right Foot } & \multicolumn{6}{|c|}{ Left Foot } \\
\hline & & & & $\mathbf{F x}$ & $\mathbf{F y}$ & $\mathbf{F z}$ & $\mathbf{M x}$ & My & Mz & $\mathbf{F x}$ & Fy & $\mathbf{F z}$ & $\mathbf{M x}$ & My & $\mathbf{M z}$ \\
\hline 1 & 5 & 30 & 1 & 46 & 143 & 669 & 76 & 37 & 1 & 50 & 130 & 663 & 42 & 43 & 11 \\
\hline 1 & 5 & 20 & 2 & 65 & 140 & 729 & 51 & 38 & 11 & 45 & 145 & 695 & 51 & 29 & 1 \\
\hline 1 & 5 & 30 & 2 & 66 & 144 & 721 & 54 & 52 & 12 & 52 & 154 & 706 & 56 & 34 & 0 \\
\hline 1 & 5 & 40 & 2 & 71 & 139 & 695 & 51 & 55 & 13 & 37 & 118 & 712 & 53 & 22 & 6 \\
\hline 1 & 10 & 20 & 1 & 31 & 189 & 643 & 68 & 48 & 14 & 44 & 198 & 619 & 65 & 16 & 10 \\
\hline 1 & 10 & 20 & 2 & 56 & 166 & 711 & 29 & 56 & 13 & 56 & 165 & 657 & 28 & 10 & 5 \\
\hline 1 & 10 & 30 & 2 & 49 & 144 & 676 & 67 & 50 & 11 & 45 & 174 & 660 & 35 & 48 & 12 \\
\hline 1 & 10 & 40 & 2 & 53 & 128 & 659 & 49 & 50 & 14 & 45 & 169 & 668 & 30 & 40 & 11 \\
\hline 2 & 5 & 20 & 1 & 60 & 92 & 638 & 26 & 34 & 8 & 87 & 92 & 714 & 39 & 30 & 5 \\
\hline 2 & 5 & 30 & 1 & 64 & 137 & 625 & 40 & 48 & 18 & 63 & 99 & 637 & 63 & 43 & 13 \\
\hline 2 & 10 & 30 & 1 & 50 & 196 & 618 & 17 & 61 & 24 & 80 & 184 & 689 & 56 & 21 & 14 \\
\hline 2 & 10 & 40 & 1 & 59 & 220 & 746 & 50 & 45 & 19 & 53 & 215 & 713 & 48 & 44 & 20 \\
\hline
\end{tabular}




\begin{tabular}{|c|c|c|c|c|c|c|c|c|c|c|c|c|c|c|c|}
\hline \multirow{2}{*}{$\begin{array}{c}\text { Subject } \\
\text { no. }\end{array}$} & \multirow{2}{*}{ Degree } & \multirow{2}{*}{ Weight } & \multirow{2}{*}{ Direction } & \multicolumn{6}{|c|}{ Right Foot } & \multicolumn{6}{|c|}{ Left Foot } \\
\hline & & & & $\mathbf{F x}$ & $\mathbf{F y}$ & $\mathbf{F z}$ & $\mathbf{M x}$ & My & $\mathbf{M z}$ & $\mathbf{F x}$ & Fy & $\mathbf{F z}$ & $\mathbf{M x}$ & My & Mz \\
\hline 2 & 10 & 20 & 2 & 60 & 150 & 693 & 75 & 35 & 11 & 100 & 229 & 672 & 70 & 53 & 19 \\
\hline 2 & 10 & 30 & 2 & 56 & 147 & 703 & 71 & 50 & 9 & 90 & 234 & 680 & 72 & 50 & 15 \\
\hline 2 & 10 & 40 & 2 & 124 & 228 & 680 & 52 & 39 & 15 & 99 & 256 & 692 & 34 & 42 & 12 \\
\hline 3 & 5 & 20 & 1 & 64 & 143 & 640 & 28 & 36 & 10 & 89 & 101 & 716 & 41 & 32 & 7 \\
\hline 3 & 5 & 30 & 1 & 63 & 169 & 630 & 35 & 43 & 13 & 58 & 87 & 632 & 58 & 38 & 8 \\
\hline 3 & 5 & 40 & 1 & 49 & 178 & 561 & 55 & 37 & 3 & 44 & 109 & 640 & 24 & 33 & 3 \\
\hline 3 & 5 & 20 & 2 & 86 & 128 & 713 & 38 & 29 & 4 & 61 & 133 & 637 & 25 & 33 & 7 \\
\hline 3 & 5 & 30 & 2 & 71 & 134 & 682 & 56 & 45 & 11 & 41 & 149 & 634 & 20 & 37 & 7 \\
\hline 3 & 5 & 40 & 2 & 63 & 125 & 630 & 59 & 42 & 11 & 54 & 130 & 624 & 235 & 33 & 10 \\
\hline 3 & 10 & 20 & 1 & 45 & 183 & 617 & 25 & 36 & 12 & 58 & 172 & 658 & 70 & 48 & 17 \\
\hline 3 & 10 & 30 & 1 & 47 & 192 & 614 & 13 & 57 & 20 & 76 & 155 & 685 & 52 & 17 & 10 \\
\hline 3 & 10 & 40 & 1 & 28 & 202 & 551 & 30 & 38 & 14 & 51 & 191 & 549 & 24 & 11 & 3 \\
\hline 3 & 10 & 20 & 2 & 58 & 170 & 650 & 70 & 48 & 17 & 44 & 180 & 610 & 20 & 36 & 12 \\
\hline 3 & 10 & 30 & 2 & 56 & 178 & 649 & 67 & 39 & 12 & 40 & 190 & 620 & 18 & 37 & 14 \\
\hline 3 & 10 & 40 & 2 & 51 & 180 & 646 & 57 & 27 & 10 & 28 & 206 & 623 & 19 & 53 & 17 \\
\hline 4 & 5 & 20 & 1 & 44 & 114 & 705 & 45 & 53 & 5 & 64 & 111 & 741 & 67 & 44 & 10 \\
\hline 4 & 5 & 30 & 1 & 39 & 126 & 717 & 65 & 66 & 9 & 58 & 116 & 661 & 45 & 36 & 4 \\
\hline 4 & 5 & 40 & 1 & 58 & 142 & 773 & 56 & 58 & 6 & 57 & 132 & 740 & 30 & 40 & 7 \\
\hline 4 & 5 & 20 & 2 & 71 & 157 & 779 & 86 & 50 & 14 & 73 & 178 & 814 & 32 & 41 & 9 \\
\hline 4 & 5 & 30 & 2 & 67 & 120 & 791 & 47 & 59 & 9 & 59 & 114 & 745 & 39 & 37 & 5 \\
\hline 4 & 5 & 40 & 2 & 71 & 148 & 809 & 79 & 33 & 12 & 65 & 159 & 801 & 32 & 47 & 9 \\
\hline 4 & 10 & 20 & 1 & 53 & 209 & 690 & 25 & 51 & 15 & 58 & 207 & 752 & 29 & 37 & 10 \\
\hline 4 & 10 & 30 & 1 & 61 & 220 & 736 & 19 & 52 & 19 & 67 & 195 & 765 & 65 & 30 & 17 \\
\hline 4 & 10 & 40 & 1 & 78 & 230 & 772 & 48 & 44 & 10 & 78 & 212 & 764 & 63 & 18 & 12 \\
\hline 4 & 10 & 20 & 2 & 79 & 205 & 775 & 71 & 50 & 19 & 66 & 230 & 754 & 38 & 28 & 11 \\
\hline 4 & 10 & 30 & 2 & 96 & 223 & 805 & 87 & 53 & 19 & 65 & 229 & 787 & 46 & 35 & 13 \\
\hline 4 & 10 & 40 & 2 & 73 & 257 & 818 & 80 & 59 & 18 & 79 & 205 & 800 & 20 & 34 & 8 \\
\hline 5 & 5 & 20 & 1 & 44 & 140 & 560 & 28 & 28 & 6 & 50 & 91 & 578 & 11 & 27 & 2 \\
\hline 5 & 5 & 30 & 1 & 70 & 138 & 620 & 75 & 22 & 6 & 70 & 129 & 620 & 21 & 29 & 4 \\
\hline 5 & 5 & 40 & 1 & 34 & 205 & 643 & 62 & 52 & 15 & 77 & 175 & 708 & 74 & 49 & 19 \\
\hline 5 & 5 & 20 & 2 & 89 & 131 & 716 & 41 & 32 & 7 & 64 & 136 & 640 & 28 & 36 & 10 \\
\hline 5 & 5 & 30 & 2 & 73 & 136 & 684 & 58 & 47 & 13 & 43 & 151 & 636 & 22 & 39 & 9 \\
\hline 5 & 5 & 40 & 2 & 64 & 155 & 636 & 59 & 42 & 11 & 55 & 135 & 629 & 30 & 34 & 10 \\
\hline
\end{tabular}




\begin{tabular}{|c|c|c|c|c|c|c|c|c|c|c|c|c|c|c|c|}
\hline \multirow{2}{*}{$\begin{array}{c}\text { Subject } \\
\text { no. }\end{array}$} & \multirow{2}{*}{ Degree } & \multirow{2}{*}{ Weight } & \multirow{2}{*}{ Direction } & \multicolumn{6}{|c|}{ Right Foot } & \multicolumn{6}{|c|}{ Left Foot } \\
\hline & & & & $\mathbf{F x}$ & $\mathbf{F y}$ & $\mathbf{F z}$ & Mx & My & $\mathbf{M z}$ & $\mathbf{F x}$ & Fy & $\mathbf{F z}$ & $\mathbf{M x}$ & My & Mz \\
\hline 5 & 10 & 20 & 1 & 63 & 189 & 562 & 64 & 43 & 12 & 39 & 193 & 528 & 25 & 19 & 6 \\
\hline 5 & 10 & 30 & 1 & 44 & 200 & 581 & 71 & 43 & 16 & 46 & 197 & 591 & 25 & 29 & 8 \\
\hline 5 & 10 & 40 & 1 & 19 & 201 & 551 & 23 & 38 & 14 & 51 & 191 & 549 & 35 & 11 & 4 \\
\hline 5 & 10 & 20 & 2 & 58 & 172 & 659 & 70 & 48 & 17 & 45 & 184 & 610 & 25 & 37 & 12 \\
\hline 5 & 10 & 30 & 2 & 76 & 155 & 685 & 52 & 17 & 10 & 47 & 192 & 614 & 12 & 57 & 20 \\
\hline 5 & 10 & 40 & 2 & 52 & 178 & 645 & 57 & 27 & 10 & 30 & 207 & 623 & 25 & 54 & 19 \\
\hline 6 & 5 & 20 & 1 & 51 & 146 & 679 & 97 & 49 & 3 & 51 & 127 & 682 & 30 & 47 & 7 \\
\hline 6 & 5 & 30 & 1 & 49 & 136 & 700 & 85 & 32 & 5 & 57 & 126 & 644 & 57 & 47 & 7 \\
\hline 6 & 5 & 40 & 1 & 46 & 158 & 638 & 77 & 46 & 10 & 48 & 127 & 656 & 57 & 46 & 12 \\
\hline 6 & 5 & 20 & 2 & 51 & 151 & 697 & 56 & 44 & 8 & 56 & 181 & 678 & 73 & 50 & 14 \\
\hline 6 & 5 & 30 & 2 & 58 & 125 & 744 & 92 & 52 & 10 & 50 & 115 & 708 & 47 & 56 & 8 \\
\hline 6 & 5 & 40 & 2 & 56 & 169 & 731 & 91 & 36 & 11 & 60 & 175 & 759 & 91 & 52 & 11 \\
\hline 6 & 10 & 20 & 1 & 40 & 214 & 670 & 64 & 45 & 13 & 70 & 173 & 663 & 33 & 37 & 9 \\
\hline 6 & 10 & 30 & 1 & 32 & 217 & 678 & 60 & 38 & 15 & 56 & 190 & 605 & 22 & 47 & 14 \\
\hline 6 & 10 & 40 & 1 & 48 & 234 & 660 & 75 & 56 & 25 & 66 & 173 & 641 & 61 & 20 & 7 \\
\hline 6 & 10 & 20 & 2 & 51 & 151 & 697 & 56 & 44 & 8 & 56 & 181 & 678 & 72 & 50 & 14 \\
\hline 6 & 10 & 30 & 2 & 54 & 149 & 682 & 72 & 47 & 7 & 50 & 179 & 661 & 40 & 53 & 17 \\
\hline 6 & 10 & 40 & 2 & 58 & 133 & 664 & 54 & 47 & 11 & 50 & 174 & 673 & 32 & 45 & 16 \\
\hline 7 & 5 & 20 & 1 & 64 & 169 & 725 & 98 & 28 & 5 & 62 & 125 & 643 & 35 & 36 & 6 \\
\hline 7 & 5 & 30 & 1 & 61 & 174 & 731 & 85 & 38 & 3 & 59 & 141 & 687 & 22 & 41 & 5 \\
\hline 7 & 5 & 40 & 1 & 62 & 180 & 699 & 85 & 34 & 4 & 52 & 147 & 664 & 24 & 34 & 5 \\
\hline 7 & 5 & 20 & 2 & 67 & 123 & 793 & 65 & 39 & 6 & 98 & 186 & 821 & 48 & 40 & 6 \\
\hline 7 & 5 & 30 & 2 & 71 & 116 & 748 & 67 & 38 & 6 & 86 & 175 & 800 & 68 & 42 & 8 \\
\hline 7 & 5 & 40 & 2 & 79 & 136 & 792 & 72 & 36 & 5 & 91 & 186 & 773 & 40 & 37 & 8 \\
\hline 7 & 10 & 20 & 1 & 50 & 192 & 655 & 88 & 39 & 6 & 71 & 160 & 648 & 37 & 31 & 7 \\
\hline 7 & 10 & 30 & 1 & 54 & 219 & 674 & 77 & 36 & 4 & 68 & 166 & 632 & 44 & 37 & 11 \\
\hline 7 & 10 & 40 & 1 & 53 & 231 & 657 & 57 & 32 & 11 & 67 & 184 & 677 & 85 & 30 & 7 \\
\hline 7 & 10 & 20 & 2 & 57 & 147 & 841 & 72 & 32 & 8 & 104 & 226 & 801 & 67 & 50 & 16 \\
\hline 7 & 10 & 30 & 2 & 53 & 144 & 814 & 68 & 47 & 6 & 93 & 211 & 837 & 69 & 47 & 12 \\
\hline 7 & 10 & 40 & 2 & 65 & 122 & 753 & 50 & 26 & 10 & 78 & 193 & 856 & 105 & 27 & 7 \\
\hline 8 & 5 & 20 & 1 & 51 & 157 & 664 & 41 & 22 & 5 & 63 & 116 & 668 & 74 & 34 & 9 \\
\hline 8 & 5 & 30 & 1 & 57 & 172 & 641 & 62 & 34 & 6 & 77 & 133 & 647 & 32 & 7 & 6 \\
\hline 8 & 5 & 40 & 1 & 75 & 185 & 711 & 21 & 31 & 4 & 77 & 153 & 736 & 25 & 15 & 5 \\
\hline
\end{tabular}




\begin{tabular}{|c|c|c|c|c|c|c|c|c|c|c|c|c|c|c|c|}
\hline \multirow{2}{*}{$\begin{array}{c}\text { Subject } \\
\text { no. }\end{array}$} & \multirow{2}{*}{ Degree } & \multirow{2}{*}{ Weight } & \multirow{2}{*}{ Direction } & \multicolumn{6}{|c|}{ Right Foot } & \multicolumn{6}{|c|}{ Left Foot } \\
\hline & & & & $\mathbf{F x}$ & $\mathbf{F y}$ & $\mathbf{F z}$ & $\mathbf{M x}$ & My & Mz & $\mathbf{F x}$ & $\mathbf{F y}$ & $\mathbf{F z}$ & $\mathbf{M x}$ & My & $\mathbf{M z}$ \\
\hline 8 & 5 & 20 & 2 & 63 & 110 & 719 & 36 & 38 & 6 & 84 & 145 & 726 & 22 & 22 & 7 \\
\hline 8 & 5 & 30 & 2 & 83 & 119 & 717 & 76 & 40 & 12 & 94 & 193 & 771 & 38 & 4 & 8 \\
\hline 8 & 5 & 40 & 2 & 69 & 109 & 743 & 56 & 31 & 7 & 76 & 176 & 776 & 25 & 30 & 10 \\
\hline 8 & 10 & 20 & 1 & 58 & 172 & 636 & 42 & 34 & 19 & 68 & 170 & 702 & 67 & 24 & 7 \\
\hline 8 & 10 & 30 & 1 & 41 & 217 & 658 & 50 & 35 & 19 & 68 & 196 & 645 & 18 & 22 & 5 \\
\hline 8 & 10 & 40 & 1 & 65 & 193 & 753 & 13 & 44 & 21 & 56 & 214 & 703 & 51 & 25 & 6 \\
\hline 8 & 10 & 20 & 2 & 72 & 140 & 733 & 78 & 37 & 5 & 73 & 166 & 753 & 55 & 38 & 25 \\
\hline 8 & 10 & 30 & 2 & 56 & 128 & 682 & 63 & 35 & 5 & 54 & 168 & 703 & 36 & 28 & 17 \\
\hline 8 & 10 & 40 & 2 & 67 & 184 & 763 & 73 & 42 & 7 & 42 & 162 & 662 & 45 & 31 & 15 \\
\hline 9 & 5 & 20 & 1 & 39 & 109 & 700 & 40 & 48 & 1 & 59 & 106 & 736 & 62 & 39 & 5 \\
\hline 9 & 5 & 30 & 1 & 41 & 128 & 719 & 67 & 68 & 11 & 60 & 118 & 663 & 47 & 38 & 6 \\
\hline 9 & 5 & 40 & 1 & 62 & 146 & 777 & 60 & 62 & 10 & 61 & 136 & 744 & 34 & 44 & 11 \\
\hline 9 & 5 & 20 & 2 & 67 & 156 & 725 & 65 & 39 & 6 & 98 & 175 & 734 & 48 & 40 & 6 \\
\hline 9 & 5 & 30 & 2 & 71 & 143 & 719 & 67 & 38 & 6 & 86 & 193 & 700 & 68 & 42 & 8 \\
\hline 9 & 5 & 40 & 2 & 79 & 146 & 680 & 72 & 36 & 5 & 91 & 206 & 689 & 40 & 37 & 8 \\
\hline 9 & 10 & 20 & 1 & 61 & 217 & 698 & 33 & 59 & 23 & 66 & 215 & 760 & 37 & 45 & 18 \\
\hline 9 & 10 & 30 & 1 & 62 & 221 & 737 & 20 & 53 & 20 & 68 & 196 & 766 & 66 & 31 & 18 \\
\hline 9 & 10 & 40 & 1 & 61 & 257 & 743 & 47 & 42 & 16 & 50 & 212 & 710 & 45 & 41 & 17 \\
\hline 9 & 10 & 20 & 2 & 57 & 147 & 690 & 72 & 32 & 8 & 104 & 236 & 689 & 67 & 50 & 16 \\
\hline 9 & 10 & 30 & 2 & 53 & 144 & 700 & 68 & 47 & 6 & 93 & 231 & 677 & 69 & 47 & 12 \\
\hline 9 & 10 & 40 & 2 & 121 & 225 & 677 & 49 & 36 & 12 & 98 & 253 & 669 & 31 & 39 & 9 \\
\hline 10 & 5 & 20 & 1 & 51 & 132 & 631 & 105 & 51 & 3 & 62 & 134 & 668 & 45 & 12 & 4 \\
\hline 10 & 5 & 30 & 1 & 89 & 168 & 663 & 62 & 46 & 9 & 51 & 174 & 670 & 70 & 41 & 13 \\
\hline 10 & 5 & 40 & 1 & 71 & 180 & 670 & 110 & 104 & 24 & 64 & 228 & 738 & 76 & 46 & 12 \\
\hline 10 & 5 & 20 & 2 & 117 & 148 & 850 & 39 & 50 & 4 & 85 & 223 & 742 & 39 & 59 & 15 \\
\hline 10 & 5 & 30 & 2 & 69 & 209 & 825 & 84 & 46 & 16 & 101 & 240 & 770 & 37 & 51 & 22 \\
\hline 10 & 5 & 40 & 2 & 78 & 217 & 884 & 90 & 81 & 24 & 111 & 287 & 790 & 80 & 32 & 12 \\
\hline 10 & 10 & 20 & 1 & 51 & 200 & 682 & 71 & 52 & 17 & 91 & 188 & 766 & 87 & 32 & 13 \\
\hline 10 & 10 & 30 & 1 & 70 & 204 & 633 & 76 & 45 & 10 & 62 & 223 & 724 & 74 & 17 & 14 \\
\hline 10 & 10 & 40 & 1 & 67 & 283 & 704 & 79 & 67 & 23 & 82 & 314 & 700 & 104 & 33 & 14 \\
\hline 10 & 10 & 20 & 2 & 80 & 306 & 647 & 70 & 76 & 16 & 88 & 275 & 675 & 22 & 76 & 34 \\
\hline 10 & 10 & 30 & 2 & 105 & 244 & 645 & 52 & 110 & 17 & 107 & 289 & 678 & 41 & 81 & 31 \\
\hline 10 & 10 & 40 & 2 & 93 & 268 & 678 & 78 & 66 & 8 & 98 & 300 & 661 & 77 & 89 & 45 \\
\hline
\end{tabular}




\begin{tabular}{|c|c|c|c|c|c|c|c|c|c|c|c|c|c|c|c|}
\hline \multirow{2}{*}{$\begin{array}{c}\text { Subject } \\
\text { no. }\end{array}$} & \multirow{2}{*}{ Degree } & \multirow{2}{*}{ Weight } & \multirow{2}{*}{ Direction } & \multicolumn{6}{|c|}{ Right Foot } & \multicolumn{6}{|c|}{ Left Foot } \\
\hline & & & & $\mathbf{F x}$ & Fy & $\mathbf{F z}$ & $\mathbf{M x}$ & My & $\mathbf{M z}$ & $\mathbf{F x}$ & $\mathbf{F y}$ & $\mathbf{F z}$ & $\mathbf{M x}$ & My & Mz \\
\hline 11 & 5 & 20 & 1 & 48 & 116 & 679 & 96 & 58 & 5 & 56 & 128 & 682 & 45 & 48 & 8 \\
\hline 11 & 5 & 30 & 1 & 50 & 136 & 689 & 88 & 54 & 6 & 56 & 127 & 644 & 56 & 49 & 9 \\
\hline 11 & 5 & 40 & 1 & 47 & 158 & 668 & 76 & 45 & 7 & 49 & 130 & 656 & 55 & 47 & 11 \\
\hline 11 & 5 & 20 & 2 & 52 & 152 & 697 & 54 & 45 & 9 & 60 & 181 & 678 & 74 & 51 & 13 \\
\hline 11 & 5 & 30 & 2 & 57 & 89 & 744 & 90 & 51 & 11 & 49 & 115 & 708 & 99 & 54 & 9 \\
\hline 11 & 5 & 40 & 2 & 59 & 167 & 731 & 87 & 38 & 12 & 59 & 175 & 759 & 89 & 50 & 12 \\
\hline 11 & 10 & 20 & 1 & 45 & 214 & 670 & 63 & 55 & 12 & 72 & 171 & 663 & 34 & 39 & 10 \\
\hline 11 & 10 & 30 & 1 & 33 & 217 & 678 & 59 & 48 & 14 & 55 & 187 & 605 & 25 & 46 & 13 \\
\hline 11 & 10 & 40 & 1 & 50 & 234 & 660 & 73 & 55 & 23 & 64 & 175 & 641 & 60 & 23 & 8 \\
\hline 11 & 10 & 20 & 2 & 52 & 154 & 697 & 59 & 45 & 9 & 55 & 161 & 670 & 69 & 49 & 13 \\
\hline 11 & 10 & 30 & 2 & 55 & 156 & 682 & 70 & 49 & 8 & 49 & 179 & 661 & 42 & 53 & 16 \\
\hline 11 & 10 & 40 & 2 & 59 & 137 & 664 & 53 & 46 & 11 & 49 & 174 & 673 & 30 & 46 & 17 \\
\hline 12 & 5 & 20 & 1 & 48 & 156 & 699 & 93 & 42 & 4 & 42 & 104 & 635 & 28 & 45 & 6 \\
\hline 12 & 5 & 30 & 1 & 51 & 148 & 674 & 81 & 42 & 6 & 55 & 135 & 668 & 47 & 40 & 8 \\
\hline 12 & 5 & 40 & 1 & 60 & 181 & 625 & 35 & 42 & 11 & 49 & 146 & 610 & 53 & 25 & 7 \\
\hline 12 & 5 & 20 & 2 & 70 & 155 & 734 & 56 & 35 & 8 & 50 & 160 & 690 & 56 & 34 & 6 \\
\hline 12 & 5 & 30 & 2 & 71 & 149 & 726 & 59 & 49 & 9 & 57 & 159 & 701 & 61 & 39 & 5 \\
\hline 12 & 5 & 40 & 2 & 76 & 122 & 700 & 56 & 52 & 10 & 42 & 123 & 737 & 58 & 27 & 11 \\
\hline 12 & 10 & 20 & 1 & 36 & 194 & 648 & 73 & 53 & 19 & 49 & 203 & 624 & 70 & 13 & 7 \\
\hline 12 & 10 & 30 & 1 & 42 & 209 & 602 & 39 & 55 & 22 & 57 & 209 & 596 & 78 & 8 & 6 \\
\hline 12 & 10 & 40 & 1 & 34 & 236 & 596 & 30 & 44 & 25 & 65 & 193 & 595 & 80 & 9 & 8 \\
\hline 12 & 10 & 20 & 2 & 61 & 181 & 716 & 34 & 53 & 10 & 61 & 160 & 672 & 33 & 15 & 10 \\
\hline 12 & 10 & 30 & 2 & 54 & 149 & 681 & 72 & 47 & 8 & 50 & 179 & 673 & 40 & 53 & 17 \\
\hline 12 & 10 & 40 & 2 & 58 & 133 & 664 & 54 & 47 & 11 & 50 & 174 & 673 & 35 & 45 & 16 \\
\hline
\end{tabular}




\section{APPENDIX G: RAW DATA OF MAJOR JOINT ANGLES}


Table G-1: Raw major joint angle data in Session 1

\begin{tabular}{|c|c|c|c|c|c|c|c|}
\hline \multirow{2}{*}{$\begin{array}{c}\text { Subject } \\
\text { no. }\end{array}$} & \multirow{2}{*}{ Weight } & Trunk & Right - Shoulder & Right - Elbow & Right - Hip & Right - Knee & Right - Ankle \\
\hline & & Flexion/extension & Flexion/extension & Flexion/extension & Flexion/extension & Flexion/extension & Flexion/extension \\
\hline 1 & 20 & 2.5 & 14.2 & 30.7 & -35 & 48.1 & 15 \\
\hline 1 & 30 & 17.1 & 32.2 & 37.1 & 40.6 & 58.3 & 12.1 \\
\hline 1 & 40 & 9.5 & -23.2 & 35.7 & 40.4 & 52.1 & 14.3 \\
\hline 2 & 20 & 2.9 & 19.7 & 58.3 & 23.1 & 62.2 & -13.2 \\
\hline 2 & 30 & 4.5 & 18.1 & 61.1 & 27.4 & 64.1 & 12 \\
\hline 2 & 40 & 6.6 & 19.4 & 55.2 & 27 & 63.9 & 11.9 \\
\hline 3 & 20 & -4.2 & 9 & 43.9 & 29.3 & 44.9 & 9.2 \\
\hline 3 & 30 & -7.3 & 8 & 44.7 & 29.5 & 47.6 & 16.1 \\
\hline 3 & 40 & -5.5 & 13.6 & 44 & 31.5 & 56.8 & 16.3 \\
\hline 4 & 20 & 2.9 & 19.6 & 62 & 23.7 & 62.5 & 9.8 \\
\hline 4 & 30 & 5.1 & 18.1 & 60.9 & 32.6 & 65.4 & 11.7 \\
\hline 4 & 40 & 7 & 24.6 & 58.1 & 29.9 & 65.3 & -14.7 \\
\hline 5 & 20 & 14.7 & 32.8 & 95 & 14.3 & 52 & 13 \\
\hline 5 & 30 & 16.7 & 26 & 116.9 & 11.1 & 53 & 15.1 \\
\hline 5 & 40 & 13.2 & 11.1 & 54.1 & 10.9 & 50 & 11.6 \\
\hline 6 & 20 & 13.4 & 26.1 & 96.2 & 12.7 & 49.6 & 13.3 \\
\hline 6 & 30 & 16.4 & 30.7 & 102.2 & 14.4 & 55.4 & 14.8 \\
\hline 6 & 40 & 19.7 & 35.6 & 108.6 & 16.3 & 61 & 16.9 \\
\hline 7 & 20 & -7.6 & -18 & 91.4 & 21.3 & 45.1 & 19.2 \\
\hline 7 & 30 & 5.4 & -17.6 & 85 & 18.5 & 47.8 & 18.8 \\
\hline 7 & 40 & -10.4 & -16 & 68.3 & 21 & 41.7 & 16.6 \\
\hline 8 & 20 & -8.3 & -18.5 & 80.3 & 29.2 & 39.2 & -15.9 \\
\hline 8 & 30 & -7.1 & -16.2 & 77 & 32.4 & 47.1 & -17.2 \\
\hline 8 & 40 & -8.1 & -8.8 & 73.8 & 33.4 & 49.3 & -18.6 \\
\hline 9 & 20 & 17.7 & 19.6 & 53.6 & 15 & 59.8 & -15.7 \\
\hline 9 & 30 & 18.1 & 14.7 & 58.2 & 16 & 62.7 & 14.6 \\
\hline 9 & 40 & 16.9 & 17 & 54.1 & 18 & 64 & 14.7 \\
\hline 10 & 20 & 5.5 & -6.2 & 99.1 & 22.9 & 46.1 & 18.1 \\
\hline 10 & 30 & -5 & -20.5 & 87.4 & 20 & 50.4 & 15.7 \\
\hline 10 & 40 & 4.2 & -21.5 & 114.4 & 20.8 & 49.7 & 17.1 \\
\hline
\end{tabular}




\begin{tabular}{|c|c|c|c|c|c|c|c|}
\hline \multirow{2}{*}{$\begin{array}{c}\text { Subject } \\
\text { no. }\end{array}$} & \multirow{2}{*}{ Weight } & Trunk & Right - Shoulder & Right - Elbow & Right - Hip & Right - Knee & Right - Ankle \\
\hline & & Flexion/extension & Flexion/extension & Flexion/extension & Flexion/extension & Flexion/extension & Flexion/extension \\
\hline 11 & 20 & 8.1 & 24.7 & 58.2 & 17.5 & 60.7 & 20 \\
\hline 11 & 30 & 8 & 21.9 & 60.6 & 18.1 & 60.4 & 20.4 \\
\hline 11 & 40 & 8 & 19 & 69.7 & 18.6 & 60.2 & 20.6 \\
\hline 12 & 20 & 4 & 32.9 & 39.1 & 21.4 & 63.2 & 18.7 \\
\hline 12 & 30 & 5.7 & 28.2 & 43.7 & 21.6 & 61.9 & 19.6 \\
\hline 12 & 40 & 6.6 & 20.2 & 53.3 & 21.3 & 60.1 & 20.3 \\
\hline
\end{tabular}

Table G-2: Raw major joint angle data in Session 2

\begin{tabular}{|c|c|c|c|c|c|c|c|c|c|}
\hline \multirow{2}{*}{$\begin{array}{c}\text { Subject } \\
\text { no. }\end{array}$} & \multirow[b]{2}{*}{ Degree } & \multirow[b]{2}{*}{ Weight } & \multirow[b]{2}{*}{ Direction } & Trunk & Right -Shoulder & Right - Elbow & Right - Hip & Right - Knee & Right - Ankle \\
\hline & & & & Flexion/extension & Flexion/extension & Flexion/extension & Flexion/extension & Flexion/extension & Flexion/extension \\
\hline 1 & 5 & 20 & 1 & 6 & 21 & 39 & 47.7 & 49.9 & 19.7 \\
\hline 1 & 5 & 30 & 1 & 12.4 & 45.5 & 39 & 51.7 & 51.9 & 23.1 \\
\hline 1 & 5 & 40 & 1 & 6.4 & 29.4 & 47.1 & 53.6 & 56.5 & 24.3 \\
\hline 1 & 5 & 20 & 2 & 12.5 & 17.3 & 45.4 & 29.7 & 64.4 & -19.9 \\
\hline 1 & 5 & 30 & 2 & 21 & -172.3 & 47.7 & 36.6 & 66.2 & 23 \\
\hline 1 & 5 & 40 & 2 & 11 & 15.6 & 42.1 & 32.9 & 55.7 & -24.2 \\
\hline 1 & 10 & 20 & 1 & 6.3 & 36 & 55.4 & 59.6 & 57.3 & 30.3 \\
\hline 1 & 10 & 30 & 1 & 12.8 & 57.9 & 42.7 & 68.8 & 63.2 & -48.5 \\
\hline 1 & 10 & 40 & 1 & 14.3 & 55.9 & 57.7 & 72.2 & 62.4 & 37.9 \\
\hline 1 & 10 & 20 & 2 & 15.6 & 25.2 & 45.7 & 34.2 & 70 & -34.9 \\
\hline 1 & 10 & 30 & 2 & 23 & 36.2 & 49 & 35.9 & 71.5 & -38 \\
\hline 1 & 10 & 40 & 2 & 23.32 & 30.9 & 43.1 & 40.3 & 75.4 & -43.1 \\
\hline 2 & 5 & 20 & 1 & 13 & 35 & 68.2 & 34.6 & 65.8 & 17 \\
\hline 2 & 5 & 30 & 1 & 13.9 & 40.2 & 52.6 & 30 & 68.8 & 24.7 \\
\hline 2 & 5 & 40 & 1 & 12.4 & 40.9 & 64.4 & 31.2 & 63.5 & 21.5 \\
\hline 2 & 5 & 20 & 2 & 14.9 & 22.6 & 55.5 & 17 & 71.1 & 16.2 \\
\hline 2 & 5 & 30 & 2 & 13.8 & 21.5 & 48.3 & 18.7 & 69.6 & 15.9 \\
\hline 2 & 5 & 40 & 2 & 13.1 & 20.7 & 59.1 & 20.3 & 70.4 & 16.6 \\
\hline 2 & 10 & 20 & 1 & 10.5 & 30.3 & 81.1 & 52 & 61.5 & 28.6 \\
\hline 2 & 10 & 30 & 1 & 10.5 & 31.1 & 79.2 & 46.3 & 61 & 30.2 \\
\hline
\end{tabular}




\begin{tabular}{|c|c|c|c|c|c|c|c|c|c|}
\hline \multirow{2}{*}{$\begin{array}{c}\text { Subject } \\
\text { no. }\end{array}$} & \multirow{2}{*}{ Degree } & \multirow{2}{*}{ Weight } & \multirow{2}{*}{ Direction } & Trunk & Right -Shoulder & Right - Elbow & Right - Hip & Right - Knee & Right - Ankle \\
\hline & & & & Flexion/extension & Flexion/extension & Flexion/extension & Flexion/extension & Flexion/extension & Flexion/extension \\
\hline 2 & 10 & 40 & 1 & 9.8 & 19.2 & 89.4 & 45.9 & 62.5 & 28.7 \\
\hline 2 & 10 & 20 & 2 & 13.9 & 24.4 & 39.8 & 17.8 & 71.8 & 14.9 \\
\hline 2 & 10 & 30 & 2 & 17.8 & 22.9 & 40.5 & 23.6 & 76.9 & 18.1 \\
\hline 2 & 10 & 40 & 2 & 17.7 & 26.7 & 51 & 24.1 & 73.5 & 14 \\
\hline 3 & 5 & 20 & 1 & -4.5 & 41.2 & 41.5 & 49.4 & 51.4 & 21.8 \\
\hline 3 & 5 & 30 & 1 & -6.8 & 24.5 & 70.8 & 41.1 & 50.9 & 23.1 \\
\hline 3 & 5 & 40 & 1 & -5.8 & 38.9 & 51.5 & 44.2 & 41.3 & 26.8 \\
\hline 3 & 5 & 20 & 2 & -4.3 & 15.5 & 44.1 & 18.7 & 47.6 & 15.8 \\
\hline 3 & 5 & 30 & 2 & 3.8 & 15.8 & 39.1 & 25.7 & 52.2 & 17.8 \\
\hline 3 & 5 & 40 & 2 & 5.3 & 22 & 37.1 & 30 & 70.8 & 18.5 \\
\hline 3 & 10 & 20 & 1 & 6.7 & 53 & 55 & 51.5 & 45.1 & 35.3 \\
\hline 3 & 10 & 30 & 1 & -8.3 & 46.5 & 50.5 & 48.8 & 50.1 & 35.4 \\
\hline 3 & 10 & 40 & 1 & -5.2 & 23.9 & 88 & 66.8 & 56.9 & 38.3 \\
\hline 3 & 10 & 20 & 2 & 6.4 & 20.1 & 38.2 & 27.8 & 79.9 & 18.5 \\
\hline 3 & 10 & 30 & 2 & 9.6 & 31.4 & 35.9 & 31.9 & 87.4 & 20.7 \\
\hline 3 & 10 & 40 & 2 & 4.5 & 15.5 & 41 & 32.3 & 78.5 & 20.8 \\
\hline 4 & 5 & 20 & 1 & 13 & 35 & 71.3 & 41.1 & 67.7 & 18.4 \\
\hline 4 & 5 & 30 & 1 & 16 & 40.2 & 55.5 & 37.5 & 69.5 & 24.6 \\
\hline 4 & 5 & 40 & 1 & 12.3 & 41.2 & 64.3 & 40.5 & 66.8 & 21.7 \\
\hline 4 & 5 & 20 & 2 & 14.9 & 23.9 & 95.4 & 21.4 & 73.6 & 16.4 \\
\hline 4 & 5 & 30 & 2 & 13.8 & 20.5 & 50 & 20.6 & 71.4 & 16.1 \\
\hline 4 & 5 & 40 & 2 & 14.7 & 21.7 & 56.9 & 20.7 & 70.5 & 16.6 \\
\hline 4 & 10 & 20 & 1 & 16.4 & 41 & 80.8 & 52.7 & 78.5 & 28.5 \\
\hline 4 & 10 & 30 & 1 & 13.6 & 37 & 97.8 & 54.7 & 73.7 & 30.2 \\
\hline 4 & 10 & 40 & 1 & 10.9 & 26 & 89.6 & 54.3 & 66.9 & 29.4 \\
\hline 4 & 10 & 20 & 2 & 13.9 & 24.6 & 44.7 & 18.3 & 74.1 & 15.3 \\
\hline 4 & 10 & 30 & 2 & 21.3 & 27.7 & 40.5 & 24 & 77.4 & 18.2 \\
\hline 4 & 10 & 40 & 2 & 20.9 & 26.7 & 53.1 & 24.6 & 73.9 & 14.1 \\
\hline 5 & 5 & 20 & 1 & 11.1 & 41.2 & 108.4 & 27.4 & 51.5 & 20.6 \\
\hline 5 & 5 & 30 & 1 & 15 & 58.1 & 70.9 & 33.5 & 52.3 & 22.1 \\
\hline 5 & 5 & 40 & 1 & 15.7 & 66.7 & 66 & 40.2 & 58.5 & 27.1 \\
\hline 5 & 5 & 20 & 2 & 8.5 & 10.7 & 67.6 & 11.2 & 63.8 & 18.5 \\
\hline
\end{tabular}




\begin{tabular}{|c|c|c|c|c|c|c|c|c|c|}
\hline \multirow{2}{*}{$\begin{array}{c}\text { Subject } \\
\text { no. }\end{array}$} & \multirow{2}{*}{ Degree } & \multirow{2}{*}{ Weight } & \multirow{2}{*}{ Direction } & Trunk & Right -Shoulder & Right - Elbow & Right - Hip & Right - Knee & Right - Ankle \\
\hline & & & & Flexion/extension & Flexion/extension & Flexion/extension & Flexion/extension & Flexion/extension & Flexion/extension \\
\hline 5 & 5 & 30 & 2 & 9.7 & 15.5 & 73.9 & 9 & 68.2 & 22.1 \\
\hline 5 & 5 & 40 & 2 & 11.2 & 18.9 & 69.3 & -21.3 & 59.2 & 25 \\
\hline 5 & 10 & 20 & 1 & 20 & 60.7 & 64 & 42.1 & 53.1 & 30.3 \\
\hline 5 & 10 & 30 & 1 & 22.6 & 48 & 96.5 & 47.2 & 55.2 & 34.2 \\
\hline 5 & 10 & 40 & 1 & 25.5 & 34.9 & 128.2 & 52.8 & 57.9 & 38.5 \\
\hline 5 & 10 & 20 & 2 & 16.4 & 21.3 & 89.1 & 16.6 & 79.6 & 25.4 \\
\hline 5 & 10 & 30 & 2 & 20.3 & 30.3 & 62.5 & 18 & 67.4 & 27.3 \\
\hline 5 & 10 & 40 & 2 & 14.8 & 28 & 60.1 & 15.2 & 70.7 & 24.5 \\
\hline 6 & 5 & 20 & 1 & 12.2 & 45.3 & 119.2 & 30.1 & 56.7 & 22.7 \\
\hline 6 & 5 & 30 & 1 & 16.3 & 59.4 & 72.2 & 34.8 & 53.6 & 23.4 \\
\hline 6 & 5 & 40 & 1 & 18.1 & 69.1 & 68.4 & 42.6 & 60.9 & 29.5 \\
\hline 6 & 5 & 20 & 2 & 9.7 & 11.9 & 68.8 & 12.4 & 65 & 19.7 \\
\hline 6 & 5 & 30 & 2 & 8.7 & 14.5 & 72.9 & 8 & 67.2 & 21.1 \\
\hline 6 & 5 & 40 & 2 & 6.6 & 14.3 & 64.5 & -29.7 & 66.5 & 22.7 \\
\hline 6 & 10 & 20 & 1 & 22.2 & 62.9 & 66.2 & 44.3 & 55.3 & 32.5 \\
\hline 6 & 10 & 30 & 1 & 23.5 & 48.9 & 97.4 & 48.1 & 56.1 & 35.1 \\
\hline 6 & 10 & 40 & 1 & 28.4 & 37.8 & 131.1 & 55.7 & 60.8 & 41.4 \\
\hline 6 & 10 & 20 & 2 & 16 & 20.9 & 88.7 & 16.2 & 79.2 & 25 \\
\hline 6 & 10 & 30 & 2 & 19.1 & 29.1 & 61.3 & 16.8 & 66.2 & 26.1 \\
\hline 6 & 10 & 40 & 2 & 15.6 & 28.8 & 60.9 & 16 & 71.5 & 25.3 \\
\hline 7 & 5 & 20 & 1 & 9 & -7.2 & 50 & 31 & 58.9 & 22 \\
\hline 7 & 5 & 30 & 1 & 21.6 & 37.3 & 72.2 & 41.1 & 55.2 & 24.1 \\
\hline 7 & 5 & 40 & 1 & 15 & 13.3 & 60.4 & 41.5 & 60.2 & 28.5 \\
\hline 7 & 5 & 20 & 2 & -7 & 11 & 46 & 15 & 19 & 19 \\
\hline 7 & 5 & 30 & 2 & 17.3 & 4.5 & 52.2 & 13.3 & 55.5 & 18.3 \\
\hline 7 & 5 & 40 & 2 & 15.5 & 6.5 & 48.5 & 13.2 & 63.5 & 21.8 \\
\hline 7 & 10 & 20 & 1 & 29.8 & 47 & 77.4 & 38.1 & 55.6 & 32 \\
\hline 7 & 10 & 30 & 1 & 29.7 & 45.8 & 78.1 & 54.7 & 55.8 & 36.7 \\
\hline 7 & 10 & 40 & 1 & 12.7 & 21.6 & 87.5 & 50.2 & 62.3 & 40 \\
\hline 7 & 10 & 20 & 2 & 27 & 9.9 & 60.6 & 15.5 & 58.8 & 19.1 \\
\hline 7 & 10 & 30 & 2 & 16.9 & 8.1 & 56.4 & 16.6 & 69 & 24 \\
\hline 7 & 10 & 40 & 2 & 13 & 4.5 & 48.7 & 16.7 & 61.5 & 15.5 \\
\hline
\end{tabular}




\begin{tabular}{|c|c|c|c|c|c|c|c|c|c|}
\hline \multirow{2}{*}{$\begin{array}{c}\text { Subject } \\
\text { no. }\end{array}$} & \multirow[b]{2}{*}{ Degree } & \multirow[b]{2}{*}{ Weight } & \multirow[b]{2}{*}{ Direction } & Trunk & Right -Shoulder & Right - Elbow & Right - Hip & Right - Knee & Right - Ankle \\
\hline & & & & Flexion/extension & Flexion/extension & Flexion/extension & Flexion/extension & Flexion/extension & Flexion/extension \\
\hline 8 & 5 & 20 & 1 & -2.9 & 8.9 & 69.3 & 43.7 & 53.8 & -24.5 \\
\hline 8 & 5 & 30 & 1 & 13.8 & 33.4 & 65.8 & 50.2 & 55.8 & -21.1 \\
\hline 8 & 5 & 40 & 1 & 6.7 & -5.5 & 82.3 & 53.9 & 60 & -25.1 \\
\hline 8 & 5 & 20 & 2 & -6.7 & 29.8 & 83.5 & 24.8 & 57 & -26.9 \\
\hline 8 & 5 & 30 & 2 & 3.3 & 9.1 & 59.2 & 38.3 & 69.1 & -27.4 \\
\hline 8 & 5 & 40 & 2 & 7.4 & -9.5 & 68.4 & 35.1 & 73.6 & -25.1 \\
\hline 8 & 10 & 20 & 1 & 7.3 & 16.5 & 85.9 & 64.8 & 63.1 & -27.9 \\
\hline 8 & 10 & 30 & 1 & 7.6 & -21.2 & 100 & 63.8 & 60 & -32.3 \\
\hline 8 & 10 & 40 & 1 & 9.6 & -11.9 & 111.9 & 62 & 66.2 & -47.3 \\
\hline 8 & 10 & 20 & 2 & 3.9 & -5.4 & 74.2 & 34 & 69.7 & -35.1 \\
\hline 8 & 10 & 30 & 2 & 8.9 & 22.5 & 70.8 & 38.8 & 75.3 & -33.2 \\
\hline 8 & 10 & 40 & 2 & 11.7 & 13.5 & 84.2 & 41.3 & 78.4 & -38.4 \\
\hline 9 & 5 & 20 & 1 & 30 & 28.8 & 54.3 & 24.2 & 60.6 & -22.2 \\
\hline 9 & 5 & 30 & 1 & 25.1 & 22 & 69.7 & 29 & 59.7 & 21.1 \\
\hline 9 & 5 & 40 & 1 & 40.4 & 32.7 & 60.6 & 40.3 & 59.7 & 25.1 \\
\hline 9 & 5 & 20 & 2 & 27.4 & 29.9 & 48.9 & 11.1 & 66.8 & 17.1 \\
\hline 9 & 5 & 30 & 2 & 38.8 & 34.3 & 46.7 & 18.9 & 79.3 & 23.1 \\
\hline 9 & 5 & 40 & 2 & 55.5 & 51.6 & 38.9 & 29 & 75.2 & 18.2 \\
\hline 9 & 10 & 20 & 1 & 28.6 & 21 & 77 & 35.4 & 64 & 30.7 \\
\hline 9 & 10 & 30 & 1 & 24.2 & 16.3 & 80.9 & 38.5 & 67.3 & 33.5 \\
\hline 9 & 10 & 40 & 1 & 25.4 & 29.5 & 84.3 & 52.5 & 72.2 & 32.4 \\
\hline 9 & 10 & 20 & 2 & 36.7 & 33.6 & 44.9 & 13 & 72.7 & 16.7 \\
\hline 9 & 10 & 30 & 2 & 32.8 & 35.6 & 48.3 & 21.8 & 78.1 & 17 \\
\hline 9 & 10 & 40 & 2 & 36.9 & 36.2 & 49.9 & 23.9 & 77.4 & 16.2 \\
\hline 10 & 5 & 20 & 1 & 9 & -7 & 49.8 & 30.9 & 58.9 & 22.4 \\
\hline 10 & 5 & 30 & 1 & 20.4 & 36.1 & 71 & 39.9 & 54 & 22.9 \\
\hline 10 & 5 & 40 & 1 & 13.8 & 12.1 & 59.2 & 40.3 & 59 & 27.3 \\
\hline 10 & 5 & 20 & 2 & -5.6 & 12.3 & 47.5 & 16.3 & 19.7 & 20.5 \\
\hline 10 & 5 & 30 & 2 & 17.7 & 4.9 & 52.6 & 13.7 & 55.9 & 18.7 \\
\hline 10 & 5 & 40 & 2 & 15.6 & 6.6 & 48.6 & 13.3 & 63.6 & 21.9 \\
\hline 10 & 10 & 20 & 1 & 28.9 & 46.1 & 76.5 & 37.2 & 54.7 & 31.1 \\
\hline 10 & 10 & 30 & 1 & 28.8 & 44.9 & 77.2 & 53.8 & 54.9 & 35.8 \\
\hline
\end{tabular}




\begin{tabular}{|c|c|c|c|c|c|c|c|c|c|}
\hline \multirow{2}{*}{$\begin{array}{c}\text { Subject } \\
\text { no. }\end{array}$} & \multirow[b]{2}{*}{ Degree } & \multirow[b]{2}{*}{ Weight } & \multirow[b]{2}{*}{ Direction } & Trunk & Right -Shoulder & Right - Elbow & Right - Hip & Right - Knee & Right - Ankle \\
\hline & & & & Flexion/extension & Flexion/extension & Flexion/extension & Flexion/extension & Flexion/extension & Flexion/extension \\
\hline 10 & 10 & 40 & 1 & 11.8 & 21.4 & 81.3 & 60.4 & 55.1 & 43 \\
\hline 10 & 10 & 20 & 2 & 26.1 & 9 & 59.7 & 14.6 & 57.9 & 18.2 \\
\hline 10 & 10 & 30 & 2 & 17.4 & 8.4 & 56.9 & 17 & 69.3 & 24.5 \\
\hline 10 & 10 & 40 & 2 & 12.1 & 3.4 & 47.6 & 15.6 & 60.4 & 14.4 \\
\hline 11 & 5 & 20 & 1 & 8.4 & 33.8 & 74.4 & 25.7 & 60 & 24.7 \\
\hline 11 & 5 & 30 & 1 & 9.4 & 37.3 & 73.2 & 27.1 & 59.8 & 26 \\
\hline 11 & 5 & 40 & 1 & 10.5 & 41.8 & 72.3 & 29.2 & 60 & 28.8 \\
\hline 11 & 5 & 20 & 2 & 12.8 & 24.9 & 60.9 & 14.4 & 65.3 & 24.1 \\
\hline 11 & 5 & 30 & 2 & 13.88 & 23.4 & 62.76 & -4.02 & 65.18 & 26.08 \\
\hline 11 & 5 & 40 & 2 & 14.6 & 22.4 & 64 & -16.3 & 65.1 & 27.4 \\
\hline 11 & 10 & 20 & 1 & 9.6 & 27.5 & 76.1 & 36.6 & 62.8 & 33.6 \\
\hline 11 & 10 & 30 & 1 & 8.4 & 24.7 & 80.74 & 42.84 & 63.88 & 36.84 \\
\hline 11 & 10 & 40 & 1 & 6.6 & 20.5 & 87.7 & 52.2 & 65.5 & 41.7 \\
\hline 11 & 10 & 20 & 2 & 14.7 & 21.8 & 56.7 & -14.8 & 69.8 & 26.5 \\
\hline 11 & 10 & 30 & 2 & 13.23 & 20.05 & 57.4 & 6.62 & 70.64 & 23.84 \\
\hline 11 & 10 & 40 & 2 & 12.6 & 19.3 & 57.7 & 15.8 & 71 & 22.7 \\
\hline 12 & 5 & 20 & 1 & 6.8 & 37.4 & 47.1 & 33.9 & 57.8 & 21.8 \\
\hline 12 & 5 & 30 & 1 & 16.16 & 65.48 & 43.268 & 42.22 & 55 & 26.84 \\
\hline 12 & 5 & 40 & 1 & 18.5 & 72.5 & 42.31 & 44.3 & 54.3 & 28.1 \\
\hline 12 & 5 & 20 & 2 & 8.3 & 25 & 55.9 & 19.6 & 67.2 & 19.9 \\
\hline 12 & 5 & 30 & 2 & 9.76 & 22.93 & 47.33 & 20.38 & 68.82 & 18.28 \\
\hline 12 & 5 & 40 & 2 & 10.9 & 21.3 & 40.6 & 21 & 70.1 & 17 \\
\hline 12 & 10 & 20 & 1 & 13.8 & 58 & 65.6 & 48.8 & 60.7 & 26 \\
\hline 12 & 10 & 30 & 1 & 21.41 & 75.88 & 51.50 & 57.64 & 62.52 & 28.02 \\
\hline 12 & 10 & 40 & 1 & 25.5 & 85.5 & 43.9 & 62.4 & 63.5 & 29.1 \\
\hline 12 & 10 & 20 & 2 & 14.2 & 23 & 41.3 & 23.9 & 75.2 & 19.4 \\
\hline 12 & 10 & 30 & 2 & 17.51 & 27.16 & 41.38 & 28.98 & 79.36 & 19.48 \\
\hline 12 & 10 & 40 & 2 & 18.5 & 28.4 & 41.4 & 30.5 & 80.6 & 19.5 \\
\hline
\end{tabular}




\section{APPENDIX H: RAW DATA OF LOW BACK AND SHOULDER JOINT LOADING}


Table H-1: Raw data of low back and shoulder joint loading in Session 1

\begin{tabular}{|c|c|c|c|c|c|c|c|c|c|c|c|c|c|}
\hline $\begin{array}{c}\text { Subject } \\
\text { no. }\end{array}$ & Weight & $\begin{array}{c}\text { L5S1 } \\
\text { ML }\end{array}$ & $\begin{array}{c}\text { L5S1 } \\
\text { PD }\end{array}$ & $\begin{array}{c}\text { L5S1 } \\
\text { AP }\end{array}$ & $\begin{array}{c}\text { RSC } \\
\text { ML }\end{array}$ & $\begin{array}{c}\text { RSC } \\
\text { IS } \\
\end{array}$ & $\begin{array}{c}\text { RSC } \\
\text { AP }\end{array}$ & $\begin{array}{c}\text { RAC } \\
\text { ML }\end{array}$ & $\begin{array}{c}\text { RAC } \\
\text { IS }\end{array}$ & $\begin{array}{c}\text { RAC } \\
\text { AP }\end{array}$ & $\begin{array}{c}\text { RGH } \\
\text { DIS }\end{array}$ & $\begin{array}{c}\text { RGH } \\
\text { IS } \\
\end{array}$ & $\begin{array}{c}\text { RGH } \\
\text { AP }\end{array}$ \\
\hline 1 & 20 & 107 & 889 & 339 & 53 & 86.3 & 41.8 & 245 & 283 & 302 & 522 & 284 & 244 \\
\hline 1 & 30 & 155 & 1300 & 410 & 61.3 & 99.3 & 51 & 348 & 442 & 347 & 748 & 530 & 485 \\
\hline 1 & 40 & 174 & 1730 & 516 & 68 & 118 & 51.9 & 516 & 874 & 400 & 820 & 624 & 558 \\
\hline 2 & 20 & 32.4 & 797 & 156 & 32 & 68.4 & 22.3 & 195 & 217 & 188 & 346 & 148 & 125 \\
\hline 2 & 30 & 46.4 & 998 & 192 & 38.6 & 87.5 & 25.1 & 274 & 298 & 225 & 550 & 136 & 168 \\
\hline 2 & 40 & 48.8 & 1200 & 232 & 44 & 104 & 30.9 & 317 & 368 & 276 & 683 & 178 & 208 \\
\hline 3 & 20 & 32.9 & 811 & 156 & 79.8 & 69 & 22.6 & 199 & 193 & 194 & 363 & 152 & 138 \\
\hline 3 & 30 & 45.6 & 1000 & 193 & 38.5 & 87.4 & 25.5 & 280 & 305 & 230 & 553 & 137 & 167 \\
\hline 3 & 40 & 117 & 1610 & 440 & 79 & 133 & 45 & 390 & 601 & 319 & 852 & 574 & 272 \\
\hline 4 & 20 & 11.6 & 909 & 105 & 92 & 67 & 24.4 & 169 & 421 & 134 & 386 & 307 & 107 \\
\hline 4 & 30 & 56.4 & 1180 & 188 & 135 & 110 & 29.9 & 356 & 501 & 237 & 730 & 466 & 252 \\
\hline 4 & 40 & 40.1 & 1300 & 425 & 148 & 134 & 45 & 420 & 761 & 355 & 985 & 583 & 350 \\
\hline 5 & 20 & 44.4 & 909 & 169 & 44.1 & 108 & 38.2 & 255 & 256 & 169 & 578 & 132 & 177 \\
\hline 5 & 30 & 87.5 & 1245 & 379 & 102 & 197 & 48.9 & 448 & 566 & 303 & 713 & 384 & 323 \\
\hline 5 & 40 & 76.1 & 1468 & 406 & 145 & 224 & 50.5 & 556 & 728 & 408 & 1260 & 645 & 367 \\
\hline 6 & 20 & 37.7 & 653 & 126 & 22.8 & 78 & 23.6 & 188 & 229 & 90.4 & 398 & 111 & 142 \\
\hline 6 & 30 & 44.5 & 727 & 119 & 37.1 & 92.4 & 27.9 & 220 & 257 & 107 & 477 & 167 & 169 \\
\hline 6 & 40 & 55.5 & 1000 & 179 & 58 & 102 & 33.3 & 294 & 503 & 149 & 634 & 340 & 223 \\
\hline 7 & 20 & 34.8 & 821 & 143 & 42.1 & 55.3 & 28.9 & 170 & 243 & 137 & 352 & 142 & 128 \\
\hline 7 & 30 & 52.4 & 1040 & 200 & 52.3 & 93.5 & 31.8 & 300 & 317 & 214 & 713 & 232 & 221 \\
\hline 7 & 40 & 63.9 & 1230 & 237 & 65 & 110 & 40.8 & 366 & 386 & 260 & 873 & 285 & 270 \\
\hline 8 & 20 & 28.6 & 980 & 156 & 71.8 & 80.5 & 30.9 & 267 & 362 & 204 & 573 & 370 & 197 \\
\hline 8 & 30 & 50.2 & 1040 & 160 & 113 & 136 & 36 & 311 & 498 & 204 & 614 & 469 & 215 \\
\hline 8 & 40 & 41.6 & 1300 & 321 & 129 & 91.4 & 49 & 420 & 761 & 457 & 809 & 514 & 380 \\
\hline 9 & 20 & 43.1 & 904 & 166 & 44.7 & 108 & 35.1 & 249 & 328 & 143 & 540 & 174 & 196 \\
\hline 9 & 30 & 26.7 & 1300 & 259 & 59.9 & 129 & 31.6 & 344 & 409 & 241 & 862 & 280 & 283 \\
\hline 9 & 40 & 84.6 & 1810 & 379 & 87 & 170 & 45.2 & 513 & 627 & 388 & 1290 & 503 & 378 \\
\hline 10 & 20 & 39.9 & 931 & 203 & 33.1 & 74.7 & 29.2 & 268 & 269 & 185 & 479 & 110 & 152 \\
\hline 10 & 30 & 55.1 & 907 & 182 & 47.7 & 137 & 49.8 & 340 & 454 & 217 & 802 & 274 & 262 \\
\hline 10 & 40 & 45.8 & 1770 & 347 & 67 & 156 & 55.7 & 456 & 636 & 353 & 880 & 516 & 249 \\
\hline
\end{tabular}




\begin{tabular}{|c|c|c|c|c|c|c|c|c|c|c|c|c|c|}
\hline $\begin{array}{c}\text { Subject } \\
\text { no. }\end{array}$ & Weight & $\begin{array}{c}\text { L5S1 } \\
\text { ML }\end{array}$ & $\begin{array}{c}\text { L5S1 } \\
\text { PD }\end{array}$ & $\begin{array}{c}\text { L5S1 } \\
\text { AP }\end{array}$ & $\begin{array}{c}\text { RSC } \\
\text { ML }\end{array}$ & $\begin{array}{c}\text { RSC } \\
\text { IS }\end{array}$ & $\begin{array}{c}\text { RSC } \\
\text { AP }\end{array}$ & $\begin{array}{c}\text { RAC } \\
\text { ML }\end{array}$ & $\begin{array}{c}\text { RAC } \\
\text { IS }\end{array}$ & $\begin{array}{c}\text { RAC } \\
\text { AP }\end{array}$ & $\begin{array}{c}\text { RGH } \\
\text { DIS }\end{array}$ & $\begin{array}{c}\text { RGH } \\
\text { IS }\end{array}$ & $\begin{array}{c}\text { RGH } \\
\text { AP }\end{array}$ \\
\hline 11 & 20 & 53 & 773 & 156 & 43 & 96 & 43 & 224 & 246 & 113 & 487 & 200 & 231 \\
\hline 11 & 30 & 65 & 927 & 164 & 75 & 130 & 66 & 258 & 295 & 145 & 515 & 205 & 207 \\
\hline 11 & 40 & 94.5 & 1350 & 224 & 95 & 159 & 80.3 & 321 & 550 & 196 & 681 & 387 & 270 \\
\hline 12 & 20 & 57.8 & 979 & 168 & 61.1 & 68.3 & 45.9 & 199 & 274 & 156 & 400 & 177 & 157 \\
\hline 12 & 30 & 71.4 & 1130 & 223 & 66.3 & 127.5 & 55.8 & 399 & 340 & 238 & 727 & 255 & 240 \\
\hline 12 & 40 & 92.9 & 1420 & 272 & 108 & 129 & 64.8 & 422 & 431 & 284 & 892 & 306 & 319 \\
\hline
\end{tabular}

Table H-2: Raw data of low back and shoulder joint loading in Session 2

\begin{tabular}{|c|c|c|c|c|c|c|c|c|c|c|c|c|c|c|c|}
\hline $\begin{array}{c}\text { Subject } \\
\text { no. }\end{array}$ & Degree & Weight & Direction & $\begin{array}{c}\text { L5S1 } \\
\text { ML }\end{array}$ & $\begin{array}{c}\text { L5S1 } \\
\text { PD }\end{array}$ & $\begin{array}{c}\text { L5S1 } \\
\text { AP }\end{array}$ & $\begin{array}{c}\text { RSC } \\
\text { ML }\end{array}$ & $\begin{array}{c}\text { RSC } \\
\text { IS }\end{array}$ & $\begin{array}{c}\text { RSC } \\
\text { AP }\end{array}$ & $\begin{array}{c}\text { RAC } \\
\text { ML }\end{array}$ & $\begin{array}{c}\text { RAC } \\
\text { IS }\end{array}$ & $\begin{array}{c}\text { RAC } \\
\text { AP }\end{array}$ & $\begin{array}{c}\text { RGH } \\
\text { DIS }\end{array}$ & $\begin{array}{c}\text { RGH } \\
\text { IS }\end{array}$ & $\begin{array}{c}\text { RGH } \\
\text { AP }\end{array}$ \\
\hline 1 & 5 & 20 & 1 & 79 & 1090 & 255 & 55 & 67 & 24 & 256 & 276 & 152 & 573 & 177 & 209 \\
\hline 1 & 5 & 30 & 1 & 135 & 1935 & 438 & 65 & 74 & 39 & 225 & 263 & 196 & 697 & 279 & 339 \\
\hline 1 & 5 & 40 & 1 & 171 & 2610 & 588 & 65 & 133 & 47 & 474 & 473 & 424 & 873 & 456 & 373 \\
\hline 1 & 5 & 20 & 2 & 81 & 847 & 174 & 64 & 105 & 35 & 281 & 300 & 144 & 507 & 210 & 213 \\
\hline 1 & 5 & 30 & 2 & 100 & 1210 & 266 & 58 & 101 & 40 & 399 & 468 & 269 & 962 & 335 & 335 \\
\hline 1 & 5 & 40 & 2 & 144 & 1355 & 186 & 123 & 130 & 59 & 390 & 461 & 197 & 879 & 402 & 374 \\
\hline 1 & 10 & 20 & 1 & 87 & 1080 & 264 & 38 & 69 & 45 & 321 & 334 & 161 & 741 & 281 & 323 \\
\hline 1 & 10 & 30 & 1 & 133 & 2619 & 661 & 134 & 158 & 56 & 411 & 447 & 543 & 998 & 619 & 529 \\
\hline 1 & 10 & 40 & 1 & 176 & 3890 & 953 & 172 & 242 & 70 & 856 & 748 & 671 & 1310 & 978 & 673 \\
\hline 1 & 10 & 20 & 2 & 74 & 620 & 127 & 39 & 79 & 24 & 205 & 315 & 100 & 527 & 88 & 169 \\
\hline 1 & 10 & 30 & 2 & 84 & 924 & 179 & 60 & 90 & 39 & 244 & 322 & 132 & 623 & 167 & 164 \\
\hline 1 & 10 & 40 & 2 & 90 & 819 & 178 & 42 & 70 & 38 & 243 & 269 & 124 & 508 & 142 & 422 \\
\hline 2 & 5 & 20 & 1 & 51 & 872 & 186 & 60 & 63 & 21 & 244 & 237 & 183 & 388 & 116 & 163 \\
\hline 2 & 5 & 30 & 1 & 98 & 1680 & 391 & 73 & 82 & 29 & 488 & 447 & 316 & 861 & 185 & 383 \\
\hline 2 & 5 & 40 & 1 & 125 & 1870 & 428 & 81 & 102 & 32 & 576 & 531 & 374 & 1030 & 220 & 461 \\
\hline 2 & 5 & 20 & 2 & 36 & 785 & 156 & 43 & 71 & 24 & 188 & 184 & 108 & 337 & 130 & 145 \\
\hline 2 & 5 & 30 & 2 & 70 & 1030 & 194 & 78 & 59 & 28 & 290 & 327 & 230 & 569 & 200 & 180 \\
\hline 2 & 5 & 40 & 2 & 58 & 1060 & 201 & 139 & 113 & 53 & 300 & 301 & 241 & 590 & 132 & 233 \\
\hline 2 & 10 & 20 & 1 & 86 & 1610 & 355 & 69 & 79 & 38 & 378 & 545 & 333 & 738 & 437 & 250 \\
\hline 2 & 10 & 30 & 1 & 86 & 2330 & 535 & 184 & 152 & 49 & 714 & 769 & 560 & 1060 & 649 & 461 \\
\hline 2 & 10 & 40 & 1 & 163 & 2900 & 673 & 229 & 194 & 66 & 911 & 985 & 703 & 1350 & 837 & 601 \\
\hline
\end{tabular}




\begin{tabular}{|c|c|c|c|c|c|c|c|c|c|c|c|c|c|c|c|}
\hline $\begin{array}{c}\text { Subject } \\
\text { no. }\end{array}$ & Degree & Weight & Direction & $\begin{array}{c}\text { L5S1 } \\
\text { ML }\end{array}$ & $\begin{array}{c}\text { L5S1 } \\
\text { PD }\end{array}$ & $\begin{array}{c}\text { L5S1 } \\
\text { AP }\end{array}$ & $\begin{array}{c}\text { RSC } \\
\text { ML }\end{array}$ & $\begin{array}{c}\text { RSC } \\
\text { IS }\end{array}$ & $\begin{array}{c}\text { RSC } \\
\text { AP }\end{array}$ & $\begin{array}{c}\text { RAC } \\
\text { ML }\end{array}$ & $\begin{array}{c}\text { RAC } \\
\text { IS }\end{array}$ & $\begin{array}{c}\text { RAC } \\
\text { AP }\end{array}$ & $\begin{array}{c}\text { RGH } \\
\text { DIS }\end{array}$ & $\begin{array}{c}\text { RGH } \\
\text { IS }\end{array}$ & $\begin{array}{c}\text { RGH } \\
\text { AP }\end{array}$ \\
\hline 2 & 10 & 20 & 2 & 39 & 938 & 182 & 61 & 67 & 26 & 241 & 254 & 201 & 404 & 178 & 182 \\
\hline 2 & 10 & 30 & 2 & 48 & 936 & 206 & 62 & 74 & 32 & 327 & 297 & 198 & 529 & 158 & 236 \\
\hline 2 & 10 & 40 & 2 & 52 & 954 & 161 & 35 & 56 & 42 & 284 & 267 & 161 & 512 & 135 & 225 \\
\hline 3 & 5 & 20 & 1 & 50 & 863 & 188 & 60 & 63 & 22 & 248 & 241 & 185 & 404 & 133 & 162 \\
\hline 3 & 5 & 30 & 1 & 95 & 1670 & 390 & 72 & 80 & 30 & 492 & 450 & 316 & 688 & 307 & 248 \\
\hline 3 & 5 & 40 & 1 & 116 & 1820 & 482 & 58 & 113 & 44 & 590 & 655 & 465 & 639 & 369 & 444 \\
\hline 3 & 5 & 20 & 2 & 34 & 738 & 153 & 42 & 72 & 24 & 189 & 186 & 107 & 335 & 130 & 146 \\
\hline 3 & 5 & 30 & 2 & 70 & 1030 & 194 & 77 & 58 & 28 & 295 & 332 & 232 & 569 & 202 & 180 \\
\hline 3 & 5 & 40 & 2 & 58 & 1070 & 202 & 133 & 75 & 53 & 328 & 331 & 242 & 651 & 131 & 237 \\
\hline 3 & 10 & 20 & 1 & 83 & 1525 & 367 & 65 & 59 & 36 & 367 & 595 & 215 & 589 & 538 & 214 \\
\hline 3 & 10 & 30 & 1 & 88 & 2290 & 525 & 184 & 153 & 51 & 502 & 772 & 552 & 887 & 609 & 332 \\
\hline 3 & 10 & 40 & 1 & 156 & 3294 & 836 & 191 & 171 & 50 & 633 & 1138 & 689 & 1289 & 674 & 489 \\
\hline 3 & 10 & 20 & 2 & 39 & 936 & 181 & 61 & 67 & 27 & 245 & 256 & 201 & 405 & 179 & 183 \\
\hline 3 & 10 & 30 & 2 & 49 & 933 & 205 & 60 & 74 & 33 & 332 & 302 & 200 & 530 & 158 & 237 \\
\hline 3 & 10 & 40 & 2 & 53 & 954 & 161 & 34 & 54 & 42 & 290 & 274 & 162 & 519 & 137 & 227 \\
\hline 4 & 5 & 20 & 1 & 46 & 1070 & 229 & 72 & 49 & 23 & 137 & 240 & 150 & 279 & 227 & 134 \\
\hline 4 & 5 & 30 & 1 & 52 & 1270 & 250 & 125 & 90 & 40 & 244 & 449 & 217 & 577 & 485 & 225 \\
\hline 4 & 5 & 40 & 1 & 118 & 1830 & 347 & 124 & 153 & 42 & 454 & 820 & 348 & 932 & 817 & 268 \\
\hline 4 & 5 & 20 & 2 & 54 & 1050 & 185 & 96 & 83 & 21 & 279 & 306 & 256 & 497 & 248 & 127 \\
\hline 4 & 5 & 30 & 2 & 54 & 1440 & 272 & 150 & 82 & 31 & 345 & 370 & 300 & 569 & 513 & 161 \\
\hline 4 & 5 & 40 & 2 & 81 & 3320 & 378 & 197 & 171 & 65 & 466 & 468 & 384 & 763 & 537 & 356 \\
\hline 4 & 10 & 20 & 1 & 53 & 1470 & 289 & 149 & 114 & 49 & 305 & 556 & 270 & 730 & 614 & 281 \\
\hline 4 & 10 & 30 & 1 & 48 & 2440 & 508 & 252 & 133 & 27 & 438 & 851 & 354 & 1220 & 768 & 471 \\
\hline 4 & 10 & 40 & 1 & 99 & 2920 & 671 & 262 & 180 & 42 & 590 & 1140 & 827 & 1281 & 956 & 636 \\
\hline 4 & 10 & 20 & 2 & 45 & 984 & 179 & 73 & 68 & 23 & 228 & 222 & 177 & 384 & 160 & 192 \\
\hline 4 & 10 & 30 & 2 & 60 & 987 & 228 & 65 & 70 & 30 & 292 & 250 & 180 & 499 & 143 & 226 \\
\hline 4 & 10 & 40 & 2 & 63 & 1070 & 229 & 75 & 81 & 38 & 348 & 305 & 231 & 595 & 177 & 256 \\
\hline 5 & 5 & 20 & 1 & 65 & 1460 & 361 & 100 & 101 & 21 & 289 & 375 & 284 & 420 & 341 & 216 \\
\hline 5 & 5 & 30 & 1 & 73 & 1370 & 327 & 55 & 89 & 33 & 200 & 407 & 133 & 495 & 363 & 270 \\
\hline 5 & 5 & 40 & 1 & 125 & 1310 & 366 & 49 & 131 & 32 & 206 & 455 & 310 & 903 & 422 & 337 \\
\hline 5 & 5 & 20 & 2 & 93 & 1100 & 192 & 47 & 72 & 30 & 238 & 218 & 130 & 475 & 201 & 206 \\
\hline 5 & 5 & 30 & 2 & 97 & 1630 & 322 & 80 & 59 & 37 & 201 & 229 & 135 & 501 & 203 & 208 \\
\hline 5 & 5 & 40 & 2 & 87 & 1990 & 395 & 126 & 131 & 47 & 314 & 307 & 341 & 730 & 183 & 232 \\
\hline
\end{tabular}




\begin{tabular}{|c|c|c|c|c|c|c|c|c|c|c|c|c|c|c|c|}
\hline $\begin{array}{c}\text { Subject } \\
\text { no. }\end{array}$ & Degree & Weight & Direction & $\begin{array}{c}\text { L5S1 } \\
\text { ML }\end{array}$ & $\begin{array}{c}\text { L5S1 } \\
\text { PD }\end{array}$ & $\begin{array}{c}\text { L5S1 } \\
\text { AP }\end{array}$ & $\begin{array}{c}\text { RSC } \\
\text { ML }\end{array}$ & $\begin{array}{c}\text { RSC } \\
\text { IS }\end{array}$ & $\begin{array}{c}\text { RSC } \\
\text { AP }\end{array}$ & $\begin{array}{c}\text { RAC } \\
\text { ML }\end{array}$ & $\begin{array}{c}\text { RAC } \\
\text { IS }\end{array}$ & $\begin{array}{c}\text { RAC } \\
\text { AP }\end{array}$ & $\begin{array}{c}\text { RGH } \\
\text { DIS }\end{array}$ & $\begin{array}{c}\text { RGH } \\
\text { IS }\end{array}$ & $\begin{array}{c}\text { RGH } \\
\text { AP }\end{array}$ \\
\hline 5 & 10 & 20 & 1 & 77 & 1220 & 306 & 79 & 110 & 53 & 436 & 438 & 350 & 805 & 595 & 292 \\
\hline 5 & 10 & 30 & 1 & 110 & 1520 & 379 & 125 & 107 & 41 & 631 & 523 & 475 & 951 & 653 & 390 \\
\hline 5 & 10 & 40 & 1 & 143 & 3700 & 921 & 196 & 214 & 60 & 1030 & 1017 & 708 & 1457 & 867 & 538 \\
\hline 5 & 10 & 20 & 2 & 70 & 854 & 155 & 77 & 73 & 27 & 328 & 308 & 207 & 675 & 223 & 242 \\
\hline 5 & 10 & 30 & 2 & 56 & 1040 & 193 & 109 & 130 & 42 & 342 & 403 & 209 & 758 & 275 & 354 \\
\hline 5 & 10 & 40 & 2 & 77 & 1050 & 226 & 70 & 106 & 41 & 380 & 355 & 235 & 869 & 245 & 381 \\
\hline 6 & 5 & 20 & 1 & 72 & 1240 & 264 & 60 & 115 & 37 & 322 & 375 & 230 & 584 & 294 & 230 \\
\hline 6 & 5 & 30 & 1 & 57 & 938 & 252 & 34 & 56 & 45 & 232 & 324 & 183 & 374 & 209 & 288 \\
\hline 6 & 5 & 40 & 1 & 113 & 2200 & 519 & 57 & 139 & 62 & 229 & 878 & 193 & 532 & 677 & 374 \\
\hline 6 & 5 & 20 & 2 & 57 & 1030 & 231 & 54 & 93 & 41 & 338 & 331 & 214 & 678 & 352 & 233 \\
\hline 6 & 5 & 30 & 2 & 93 & 1230 & 265 & 88 & 105 & 53 & 433 & 425 & 271 & 879 & 453 & 302 \\
\hline 6 & 5 & 40 & 2 & 125 & 2140 & 488 & 198 & 143 & 81 & 514 & 816 & 344 & 549 & 450 & 411 \\
\hline 6 & 10 & 20 & 1 & 72 & 1240 & 264 & 60 & 115 & 54 & 322 & 645 & 230 & 680 & 364 & 246 \\
\hline 6 & 10 & 30 & 1 & 111 & 2460 & 623 & 171 & 145 & 55 & 359 & 702 & 430 & 784 & 555 & 450 \\
\hline 6 & 10 & 40 & 1 & 140 & 3332 & 942 & 159 & 199 & 62 & 831 & 1127 & 609 & 1054 & 633 & 598 \\
\hline 6 & 10 & 20 & 2 & 71 & 1030 & 241 & 37 & 61 & 46 & 388 & 353 & 242 & 669 & 207 & 242 \\
\hline 6 & 10 & 30 & 2 & 66 & 1010 & 240 & 47 & 88 & 56 & 382 & 350 & 234 & 692 & 259 & 255 \\
\hline 6 & 10 & 40 & 2 & 83 & 995 & 228 & 105 & 122 & 46 & 381 & 413 & 227 & 734 & 289 & 298 \\
\hline 7 & 5 & 20 & 1 & 108 & 1370 & 362 & 94 & 66 & 31 & 198 & 290 & 142 & 448 & 262 & 179 \\
\hline 7 & 5 & 30 & 1 & 82 & 1250 & 338 & 59 & 79 & 26 & 251 & 398 & 206 & 423 & 362 & 155 \\
\hline 7 & 5 & 40 & 1 & 134 & 1420 & 254 & 61 & 169 & 32 & 443 & 253 & 354 & 563 & 208 & 199 \\
\hline 7 & 5 & 20 & 2 & 49 & 819 & 145 & 30 & 67 & 27 & 185 & 184 & 112 & 375 & 190 & 153 \\
\hline 7 & 5 & 30 & 2 & 108 & 1530 & 274 & 133 & 116 & 38 & 417 & 433 & 336 & 771 & 394 & 220 \\
\hline 7 & 5 & 40 & 2 & 132 & 3000 & 617 & 187 & 160 & 55 & 641 & 1070 & 508 & 963 & 468 & 369 \\
\hline 7 & 10 & 20 & 1 & 65 & 1460 & 362 & 79 & 131 & 51 & 428 & 430 & 347 & 787 & 272 & 223 \\
\hline 7 & 10 & 30 & 1 & 130 & 3120 & 780 & 175 & 181 & 47 & 587 & 1060 & 505 & 1126 & 732 & 425 \\
\hline 7 & 10 & 40 & 1 & 145 & 3710 & 922 & 203 & 245 & 58 & 1010 & 1290 & 874 & 1468 & 984 & 530 \\
\hline 7 & 10 & 20 & 2 & 71 & 1090 & 200 & 79 & 95 & 32 & 279 & 294 & 223 & 708 & 152 & 199 \\
\hline 7 & 10 & 30 & 2 & 98 & 1210 & 247 & 88 & 106 & 36 & 304 & 300 & 196 & 794 & 278 & 242 \\
\hline 7 & 10 & 40 & 2 & 107 & 1160 & 227 & 50 & 107 & 45 & 390 & 378 & 242 & 852 & 161 & 353 \\
\hline 8 & 5 & 20 & 1 & 46 & 1070 & 230 & 72 & 48 & 23 & 139 & 240 & 151 & 281 & 227 & 135 \\
\hline 8 & 5 & 30 & 1 & 55 & 1470 & 290 & 150 & 113 & 49 & 308 & 555 & 273 & 734 & 615 & 282 \\
\hline 8 & 5 & 40 & 1 & 118 & 1830 & 348 & 124 & 153 & 41 & 460 & 817 & 352 & 939 & 818 & 267 \\
\hline
\end{tabular}




\begin{tabular}{|c|c|c|c|c|c|c|c|c|c|c|c|c|c|c|c|}
\hline $\begin{array}{c}\text { Subject } \\
\text { no. }\end{array}$ & Degree & Weight & Direction & $\begin{array}{c}\text { L5S1 } \\
\text { ML }\end{array}$ & $\begin{array}{c}\text { L5S1 } \\
\text { PD }\end{array}$ & $\begin{array}{c}\text { L5S1 } \\
\text { AP }\end{array}$ & $\begin{array}{c}\text { RSC } \\
\text { ML }\end{array}$ & $\begin{array}{c}\text { RSC } \\
\text { IS }\end{array}$ & $\begin{array}{c}\text { RSC } \\
\text { AP }\end{array}$ & $\begin{array}{c}\text { RAC } \\
\text { ML }\end{array}$ & $\begin{array}{c}\text { RAC } \\
\text { IS }\end{array}$ & $\begin{array}{c}\text { RAC } \\
\text { AP }\end{array}$ & $\begin{array}{c}\text { RGH } \\
\text { DIS }\end{array}$ & $\begin{array}{c}\text { RGH } \\
\text { IS }\end{array}$ & $\begin{array}{c}\text { RGH } \\
\text { AP }\end{array}$ \\
\hline 8 & 5 & 20 & 2 & 54 & 1050 & 184 & 97 & 82 & 21 & 282 & 308 & 257 & 493 & 250 & 129 \\
\hline 8 & 5 & 30 & 2 & 54 & 1430 & 271 & 150 & 83 & 30 & 430 & 474 & 367 & 747 & 416 & 255 \\
\hline 8 & 5 & 40 & 2 & 107 & 2546 & 373 & 218 & 156 & 54 & 587 & 769 & 394 & 904 & 565 & 443 \\
\hline 8 & 10 & 20 & 1 & 36 & 1160 & 230 & 72 & 75 & 39 & 273 & 261 & 138 & 530 & 489 & 191 \\
\hline 8 & 10 & 30 & 1 & 49 & 2440 & 507 & 251 & 132 & 27 & 440 & 849 & 357 & 1220 & 724 & 473 \\
\hline 8 & 10 & 40 & 1 & 101 & 2900 & 668 & 260 & 205 & 43 & 1130 & 1140 & 829 & 1504 & 984 & 630 \\
\hline 8 & 10 & 20 & 2 & 45 & 986 & 178 & 73 & 68 & 24 & 231 & 222 & 178 & 384 & 160 & 190 \\
\hline 8 & 10 & 30 & 2 & 60 & 988 & 230 & 64 & 69 & 30 & 294 & 252 & 181 & 498 & 142 & 225 \\
\hline 8 & 10 & 40 & 2 & 63 & 1070 & 229 & 75 & 81 & 38 & 348 & 305 & 231 & 595 & 177 & 256 \\
\hline 9 & 5 & 20 & 1 & 40 & 1390 & 315 & 88 & 72 & 29 & 261 & 300 & 230 & 607 & 235 & 192 \\
\hline 9 & 5 & 30 & 1 & 62 & 1550 & 383 & 52 & 60 & 30 & 277 & 286 & 206 & 635 & 159 & 185 \\
\hline 9 & 5 & 40 & 1 & 100 & 2138 & 470 & 97 & 136 & 43 & 280 & 600 & 271 & 874 & 692 & 447 \\
\hline 9 & 5 & 20 & 2 & 47 & 809 & 143 & 73 & 49 & 30 & 269 & 286 & 212 & 739 & 157 & 214 \\
\hline 9 & 5 & 30 & 2 & 91 & 1530 & 277 & 165 & 99 & 34 & 277 & 283 & 186 & 830 & 277 & 248 \\
\hline 9 & 5 & 40 & 2 & 115 & 2830 & 543 & 169 & 102 & 59 & 357 & 357 & 228 & 855 & 169 & 344 \\
\hline 9 & 10 & 20 & 1 & 102 & 1390 & 352 & 55 & 109 & 49 & 442 & 500 & 195 & 594 & 339 & 233 \\
\hline 9 & 10 & 30 & 1 & 63 & 2448 & 600 & 156 & 134 & 44 & 334 & 516 & 420 & 778 & 563 & 407 \\
\hline 9 & 10 & 40 & 1 & 147 & 3333 & 816 & 184 & 161 & 54 & 767 & 927 & 601 & 1554 & 716 & 573 \\
\hline 9 & 10 & 20 & 2 & 66 & 1100 & 204 & 31 & 62 & 27 & 202 & 198 & 129 & 420 & 149 & 138 \\
\hline 9 & 10 & 30 & 2 & 94 & 1210 & 243 & 95 & 100 & 36 & 389 & 410 & 153 & 716 & 217 & 310 \\
\hline 9 & 10 & 40 & 2 & 103 & 1170 & 230 & 109 & 86 & 52 & 375 & 412 & 258 & 1024 & 332 & 323 \\
\hline 10 & 5 & 20 & 1 & 67 & 1472 & 286 & 59 & 67 & 30 & 330 & 486 & 350 & 665 & 233 & 276 \\
\hline 10 & 5 & 30 & 1 & 108 & 1860 & 388 & 40 & 113 & 47 & 564 & 531 & 387 & 858 & 300 & 319 \\
\hline 10 & 5 & 40 & 1 & 118 & 2020 & 456 & 43 & 166 & 47 & 660 & 634 & 470 & 1130 & 653 & 260 \\
\hline 10 & 5 & 20 & 2 & 80 & 1380 & 298 & 116 & 112 & 36 & 435 & 450 & 367 & 785 & 272 & 232 \\
\hline 10 & 5 & 30 & 2 & 63 & 2400 & 486 & 127 & 143 & 63 & 821 & 995 & 454 & 968 & 606 & 332 \\
\hline 10 & 5 & 40 & 2 & 93 & 3043 & 499 & 193 & 192 & 71 & 957 & 1105 & 512 & 1110 & 662 & 656 \\
\hline 10 & 10 & 20 & 1 & 93 & 2180 & 483 & 61 & 123 & 52 & 821 & 743 & 558 & 860 & 542 & 359 \\
\hline 10 & 10 & 30 & 1 & 59 & 2520 & 444 & 180 & 178 & 59 & 1080 & 1250 & 595 & 1050 & 666 & 676 \\
\hline 10 & 10 & 40 & 1 & 121 & 2820 & 899 & 150 & 201 & 85 & 1330 & 1520 & 889 & 1480 & 930 & 923 \\
\hline 10 & 10 & 20 & 2 & 56 & 1730 & 341 & 105 & 122 & 43 & 517 & 600 & 377 & 919 & 407 & 294 \\
\hline 10 & 10 & 30 & 2 & 40 & 1130 & 209 & 98 & 161 & 45 & 646 & 491 & 294 & 843 & 359 & 246 \\
\hline 10 & 10 & 40 & 2 & 75 & 2900 & 579 & 133 & 139 & 61 & 706 & 904 & 434 & 1073 & 513 & 650 \\
\hline
\end{tabular}




\begin{tabular}{|c|c|c|c|c|c|c|c|c|c|c|c|c|c|c|c|}
\hline $\begin{array}{c}\text { Subject } \\
\text { no. }\end{array}$ & Degree & Weight & Direction & $\begin{array}{c}\text { L5S1 } \\
\text { ML }\end{array}$ & $\begin{array}{c}\text { L5S1 } \\
\text { PD }\end{array}$ & $\begin{array}{c}\text { L5S1 } \\
\text { AP }\end{array}$ & $\begin{array}{c}\text { RSC } \\
\text { ML }\end{array}$ & $\begin{array}{c}\text { RSC } \\
\text { IS }\end{array}$ & $\begin{array}{c}\text { RSC } \\
\text { AP }\end{array}$ & $\begin{array}{c}\text { RAC } \\
\text { ML }\end{array}$ & $\begin{array}{c}\text { RAC } \\
\text { IS }\end{array}$ & $\begin{array}{c}\text { RAC } \\
\text { AP }\end{array}$ & $\begin{array}{c}\text { RGH } \\
\text { DIS }\end{array}$ & $\begin{array}{c}\text { RGH } \\
\text { IS }\end{array}$ & $\begin{array}{c}\text { RGH } \\
\text { AP }\end{array}$ \\
\hline 11 & 5 & 20 & 1 & 57 & 1081 & 240 & 81 & 58 & 32 & 146 & 254 & 164 & 302 & 250 & 157 \\
\hline 11 & 5 & 30 & 1 & 66 & 1304 & 264 & 129 & 94 & 44 & 248 & 467 & 235 & 591 & 499 & 239 \\
\hline 11 & 5 & 40 & 1 & 130 & 1841 & 359 & 126 & 155 & 44 & 476 & 842 & 370 & 957 & 785 & 280 \\
\hline 11 & 5 & 20 & 2 & 67 & 1053 & 198 & 109 & 87 & 25 & 283 & 309 & 259 & 510 & 261 & 140 \\
\hline 11 & 5 & 30 & 2 & 65 & 1451 & 283 & 161 & 93 & 41 & 356 & 381 & 311 & 590 & 524 & 172 \\
\hline 11 & 5 & 40 & 2 & 95 & 2981 & 393 & 205 & 133 & 70 & 476 & 478 & 394 & 748 & 552 & 371 \\
\hline 11 & 10 & 20 & 1 & 71 & 1488 & 307 & 167 & 132 & 48 & 323 & 574 & 288 & 701 & 585 & 252 \\
\hline 11 & 10 & 30 & 1 & 57 & 2469 & 517 & 261 & 138 & 52 & 447 & 860 & 363 & 1165 & 683 & 416 \\
\hline 11 & 10 & 40 & 1 & 109 & 2930 & 690 & 281 & 187 & 61 & 910 & 920 & 607 & 1190 & 893 & 616 \\
\hline 11 & 10 & 20 & 2 & 41 & 980 & 175 & 69 & 64 & 19 & 224 & 218 & 173 & 380 & 156 & 188 \\
\hline 11 & 10 & 30 & 2 & 54 & 981 & 222 & 129 & 64 & 37 & 308 & 266 & 196 & 515 & 159 & 242 \\
\hline 11 & 10 & 40 & 2 & 68 & 1125 & 234 & 80 & 86 & 43 & 353 & 318 & 244 & 608 & 190 & 269 \\
\hline 12 & 5 & 20 & 1 & 65 & 937 & 199 & 69 & 76 & 27 & 261 & 266 & 204 & 417 & 136 & 184 \\
\hline 12 & 5 & 30 & 1 & 117 & 1686 & 405 & 87 & 97 & 41 & 500 & 463 & 316 & 869 & 194 & 402 \\
\hline 12 & 5 & 40 & 1 & 144 & 1893 & 446 & 99 & 115 & 44 & 594 & 549 & 388 & 1047 & 238 & 473 \\
\hline 12 & 5 & 20 & 2 & 59 & 808 & 179 & 66 & 88 & 40 & 217 & 213 & 137 & 366 & 150 & 165 \\
\hline 12 & 5 & 30 & 2 & 107 & 1077 & 231 & 115 & 76 & 45 & 307 & 348 & 251 & 590 & 229 & 209 \\
\hline 12 & 5 & 40 & 2 & 79 & 1111 & 222 & 158 & 86 & 58 & 321 & 322 & 265 & 614 & 156 & 257 \\
\hline 12 & 10 & 20 & 1 & 105 & 1800 & 374 & 98 & 92 & 57 & 396 & 563 & 351 & 756 & 456 & 269 \\
\hline 12 & 10 & 30 & 1 & 116 & 2384 & 559 & 218 & 135 & 61 & 748 & 803 & 594 & 1064 & 658 & 470 \\
\hline 12 & 10 & 40 & 1 & 172 & 2937 & 684 & 240 & 205 & 77 & 922 & 986 & 714 & 1350 & 848 & 612 \\
\hline 12 & 10 & 20 & 2 & 48 & 977 & 191 & 70 & 76 & 35 & 250 & 263 & 210 & 433 & 187 & 199 \\
\hline 12 & 10 & 30 & 2 & 55 & 973 & 213 & 69 & 91 & 41 & 342 & 312 & 213 & 544 & 173 & 251 \\
\hline 12 & 10 & 40 & 2 & 63 & 995 & 172 & 46 & 67 & 53 & 295 & 278 & 172 & 535 & 158 & 248 \\
\hline
\end{tabular}


APPENDIX I: ANOVA TABLES FOR MODEL 1 
Table I-1: ANOVA tables for peak reaction forces acting at the L5S1 joint in three anatomical directions as a function of load weight

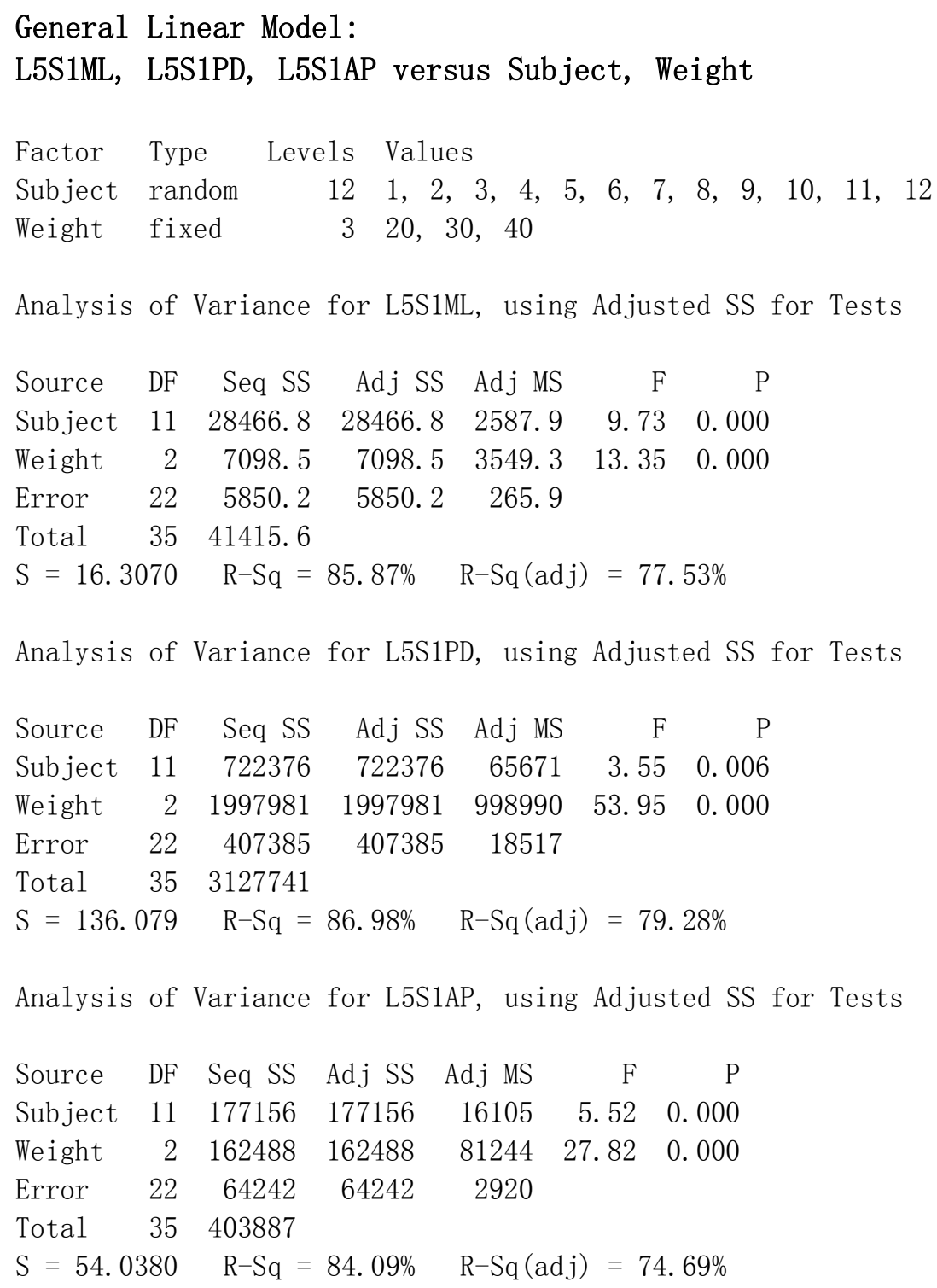

Table I-2: ANOVA tables for peak reaction forces acting at the sternoclavicular (SC) joint in three anatomical directions as a function of load weight

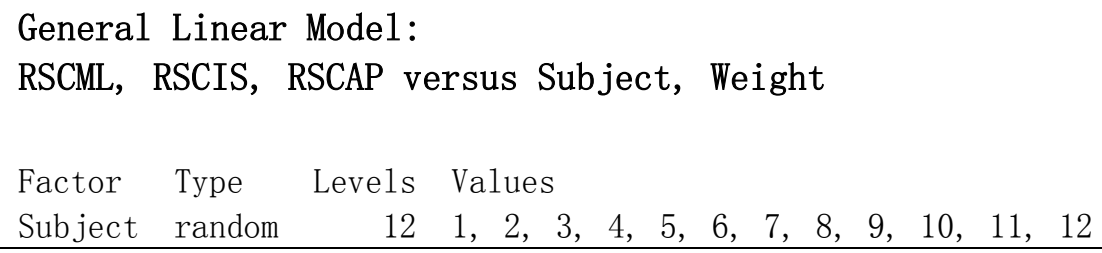




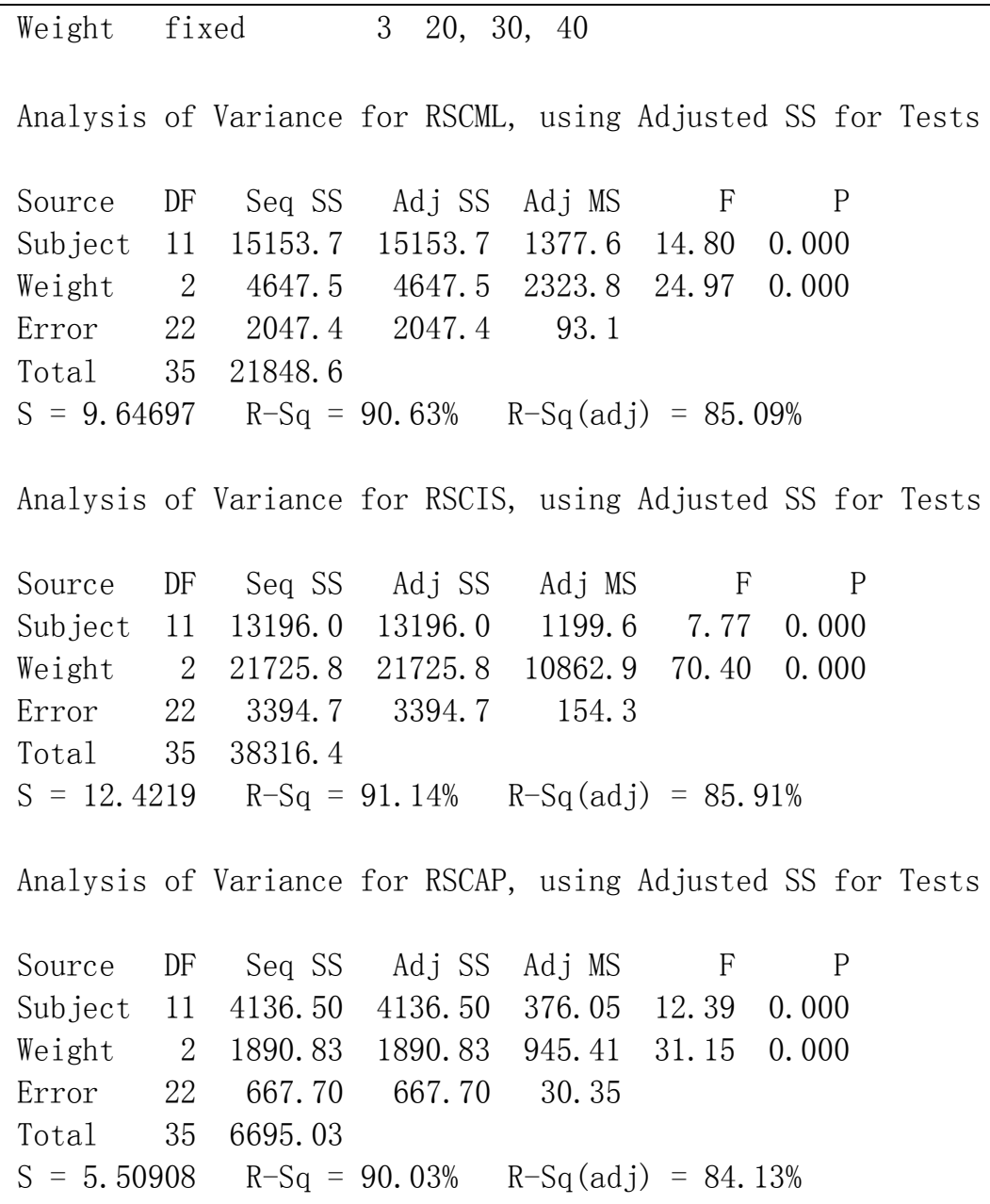

Table I-3: ANOVA tables for peak reaction forces acting at the acromioclavicular (AC) joint in three anatomical directions as a function of load weight

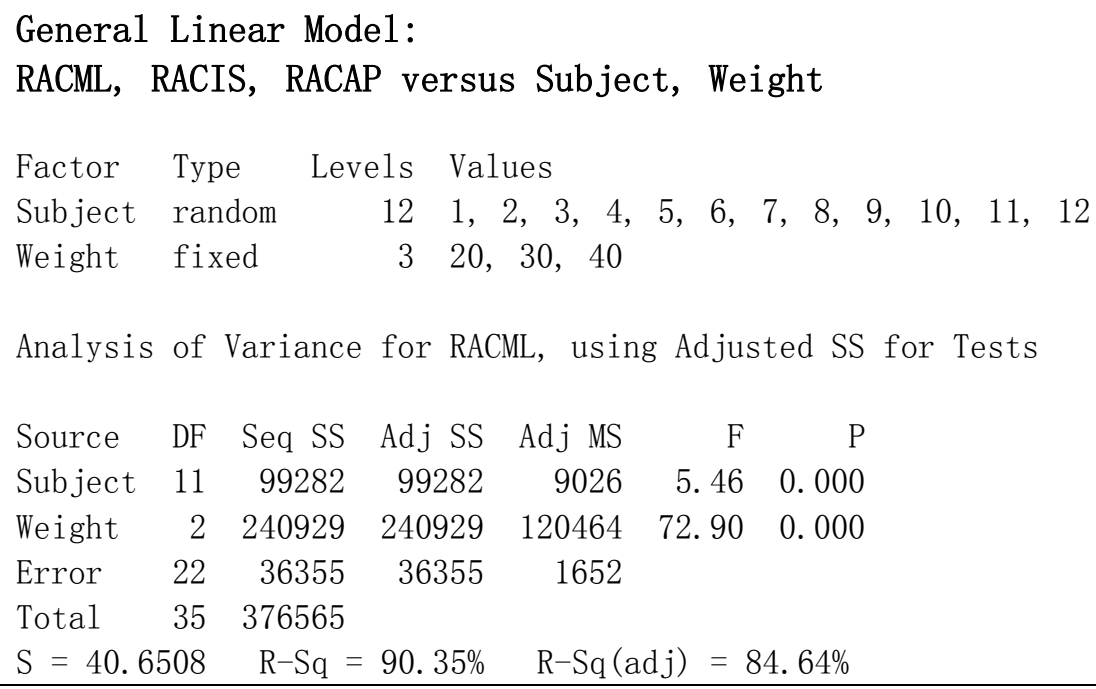




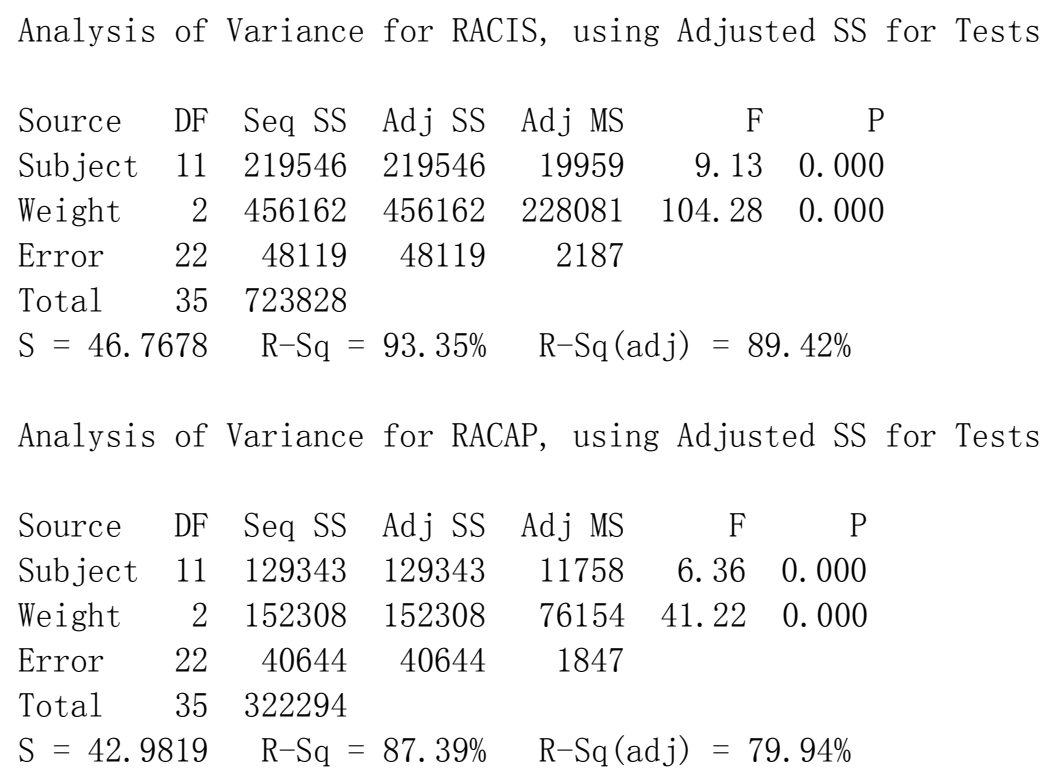

Table I-4: ANOVA tables for peak reaction forces acting at the glenohumeral (GH) joint in three anatomical directions as a function of load weight

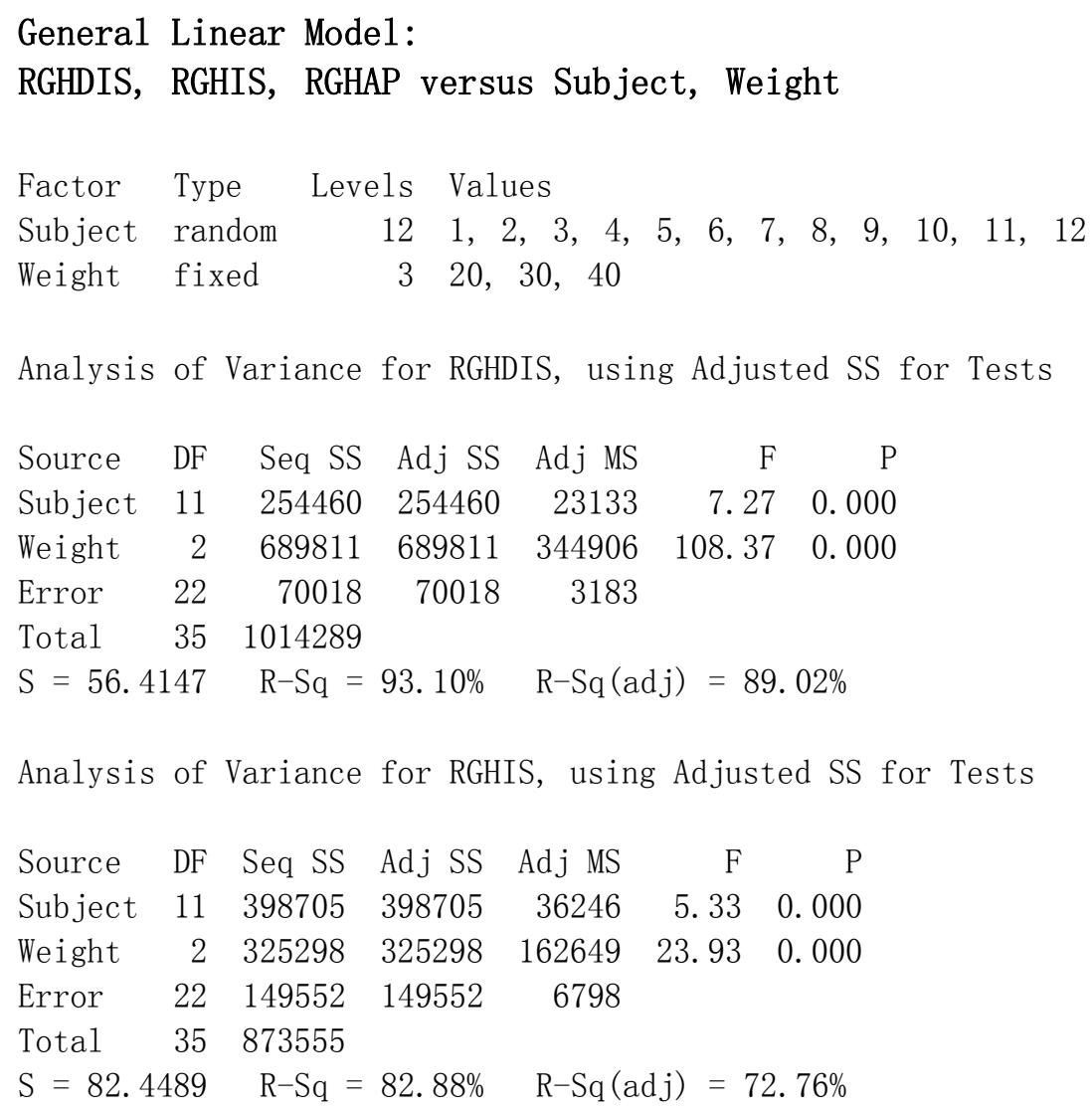




\begin{tabular}{|lrrrrrr|}
\hline \multicolumn{1}{|l}{ Analysis of Variance for RGHAP, using Adjusted SS for Tests } \\
Source & DF & Seq SS & Adj SS & Adj MS & F & P \\
Subject & 11 & 155865 & 155865 & 14170 & 6.89 & 0.000 \\
Weight & 2 & 135534 & 135534 & 67767 & 32.94 & 0.000 \\
Error & 22 & 45263 & 45263 & 2057 & & \\
Total & 35 & 336662 & & & \\
S $=45.3587$ & R-Sq $=86.56 \%$ & R-Sq (adj $)=78.61 \%$ \\
\end{tabular}


APPENDIX J: ANOVA TABLES FOR MODEL 2 
Table J-1: ANOVA tables for peak reaction forces acting at the L5S1 joint in three anatomical directions as a function of walkway gradient, load weight, and direction of force application

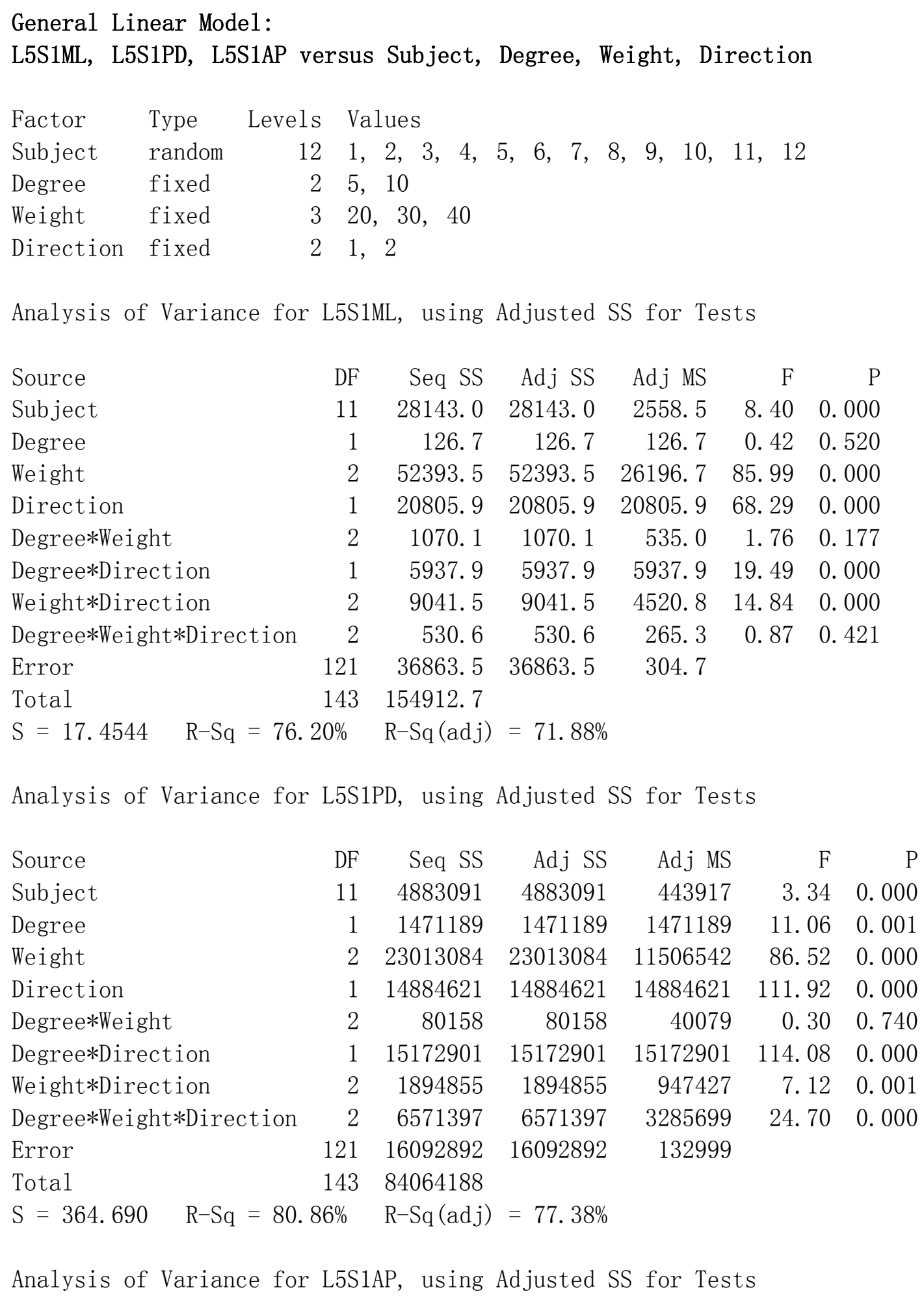




\begin{tabular}{|lrrrrrr|}
\hline Source & DF & Seq SS & Adj SS & Adj MS & F & P \\
Subject & 11 & 242687 & 242687 & 22062 & 3.53 & 0.000 \\
Degree & 1 & 227602 & 227602 & 227602 & 36.44 & 0.000 \\
Weight & 2 & 1133253 & 1133253 & 566626 & 90.72 & 0.000 \\
Direction & 1 & 1493834 & 1493834 & 1493834 & 239.18 & 0.000 \\
Degree*Weight & 2 & 42633 & 42633 & 21317 & 3.41 & 0.036 \\
Degree*Direction & 1 & 725152 & 725152 & 725152 & 116.10 & 0.000 \\
Weight*Direction & 2 & 251423 & 251423 & 125711 & 20.13 & 0.000 \\
Degree*Weight*Direction & 2 & 319680 & 319680 & 159840 & 25.59 & 0.000 \\
Error & 121 & 755738 & 755738 & 6246 & & \\
Total & 143 & 5192002 & & & & \\
S = 79.0302 & $\mathrm{R}-\mathrm{Sq}=85.44 \%$ & $\mathrm{R}-$ Sq (adj) $=82.80 \%$ & & & \\
& & & & & & \\
\hline
\end{tabular}

Table J-2: ANOVA tables for peak reaction forces acting at the sternoclavicular (SC) joint in three anatomical directions as a function of walkway gradient, load weight, and direction of force application

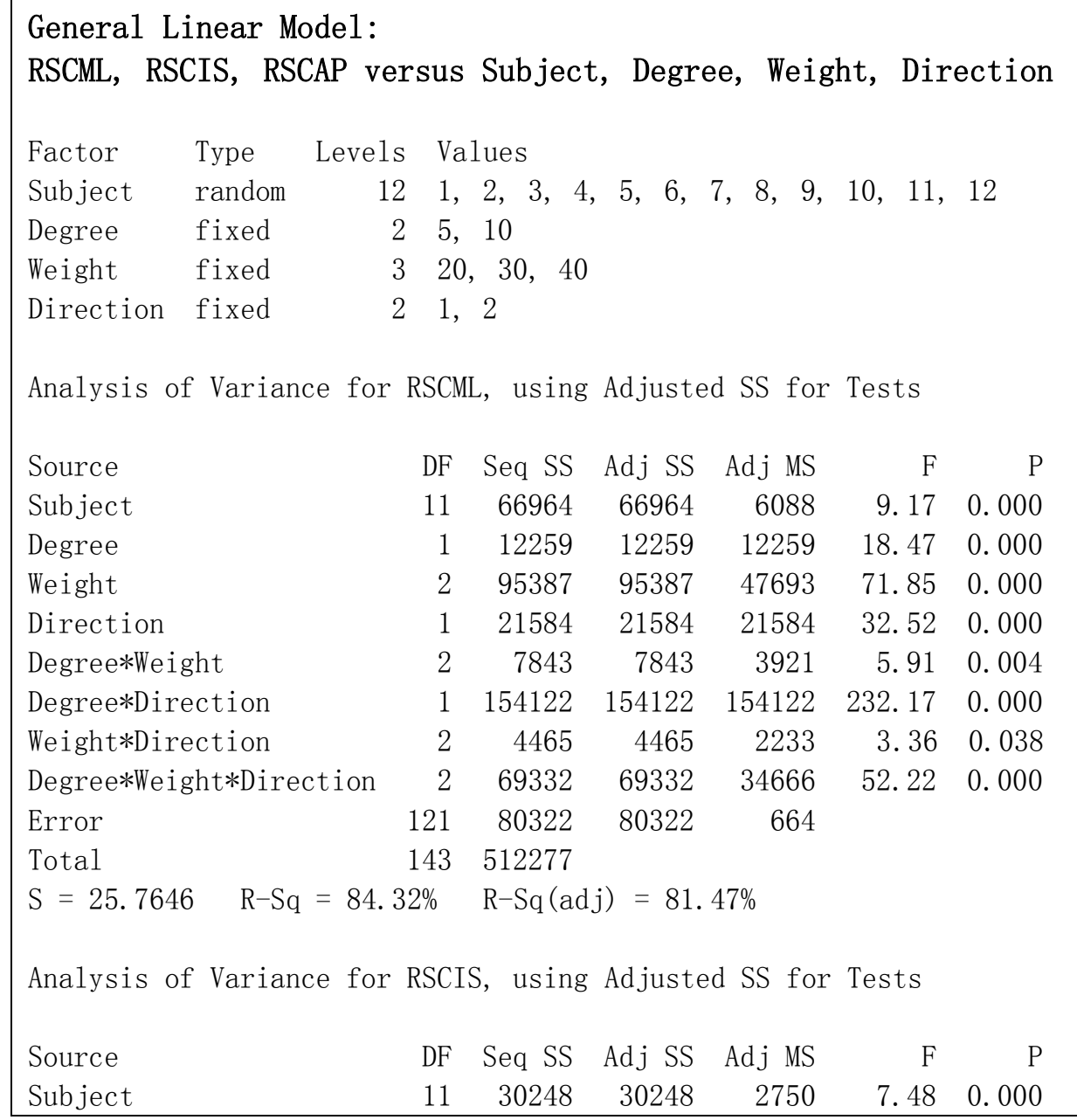




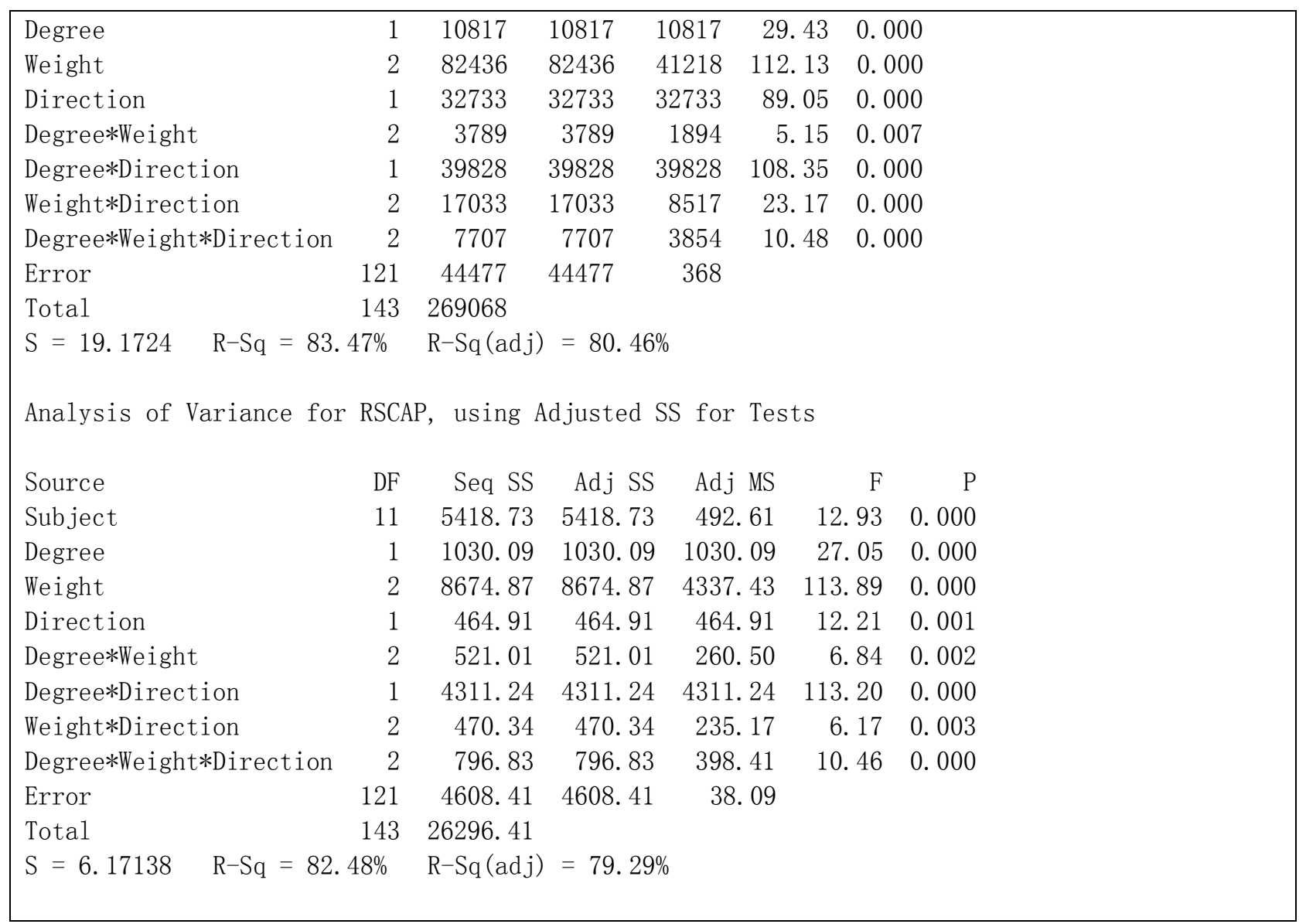

Table J-3: ANOVA tables for peak reaction forces acting at the acromioclavicular (AC) joint in three anatomical directions as a function of walkway gradient, load weight, and direction of force application

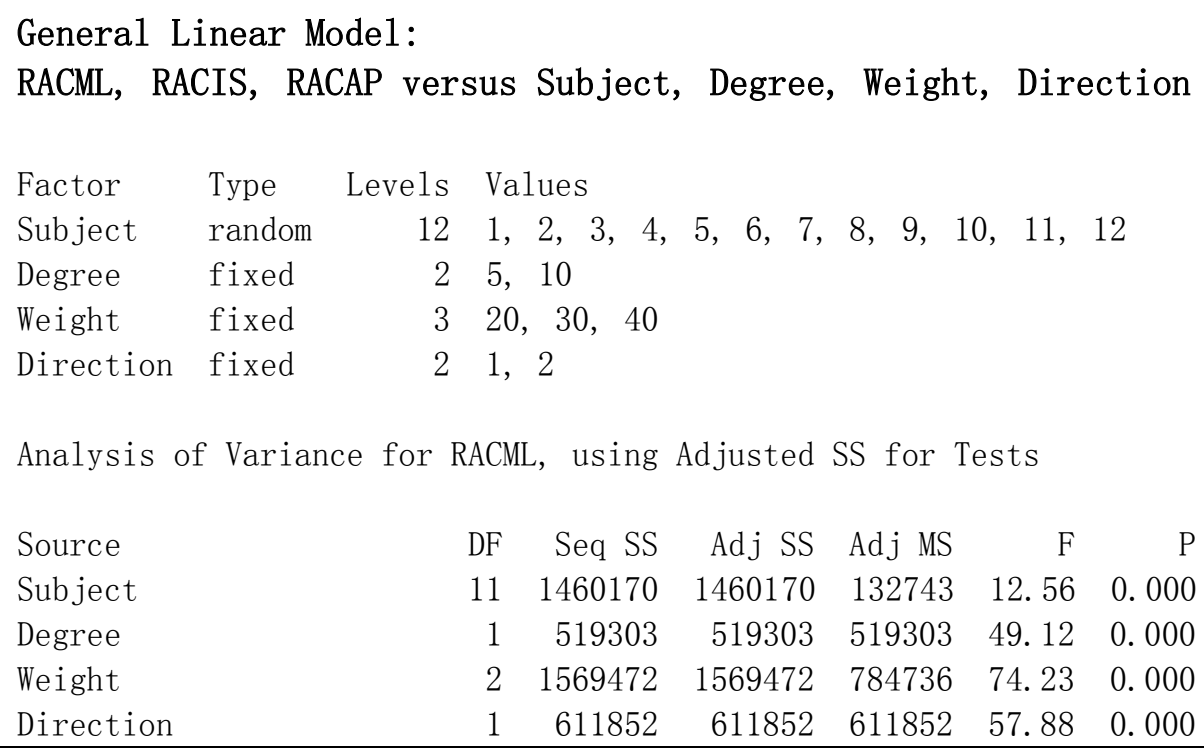




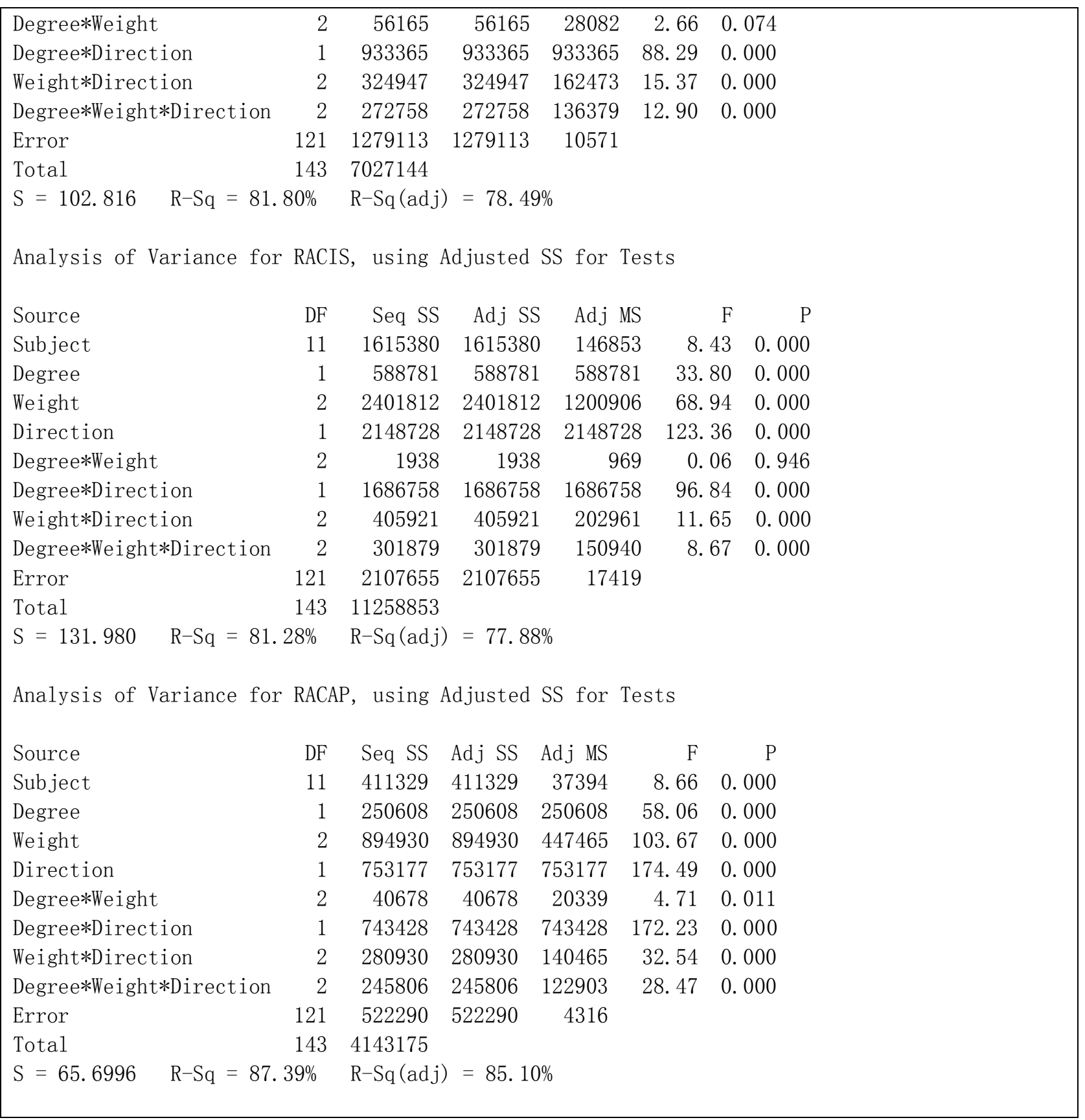

Table J-4: ANOVA tables for peak reaction forces acting at the glenohumeral $(\mathrm{GH})$ joint in three anatomical directions as a function of walkway gradient, load weight, and direction of force application

\section{General Linear Mode1: \\ RGHDIS, RGHIS, RGHAP versus Subject, Degree, Weight, Direction}

Factor Type Levels Values

Subject random $121,2,3,4,5,6,7,8,9,10,11,12$ 


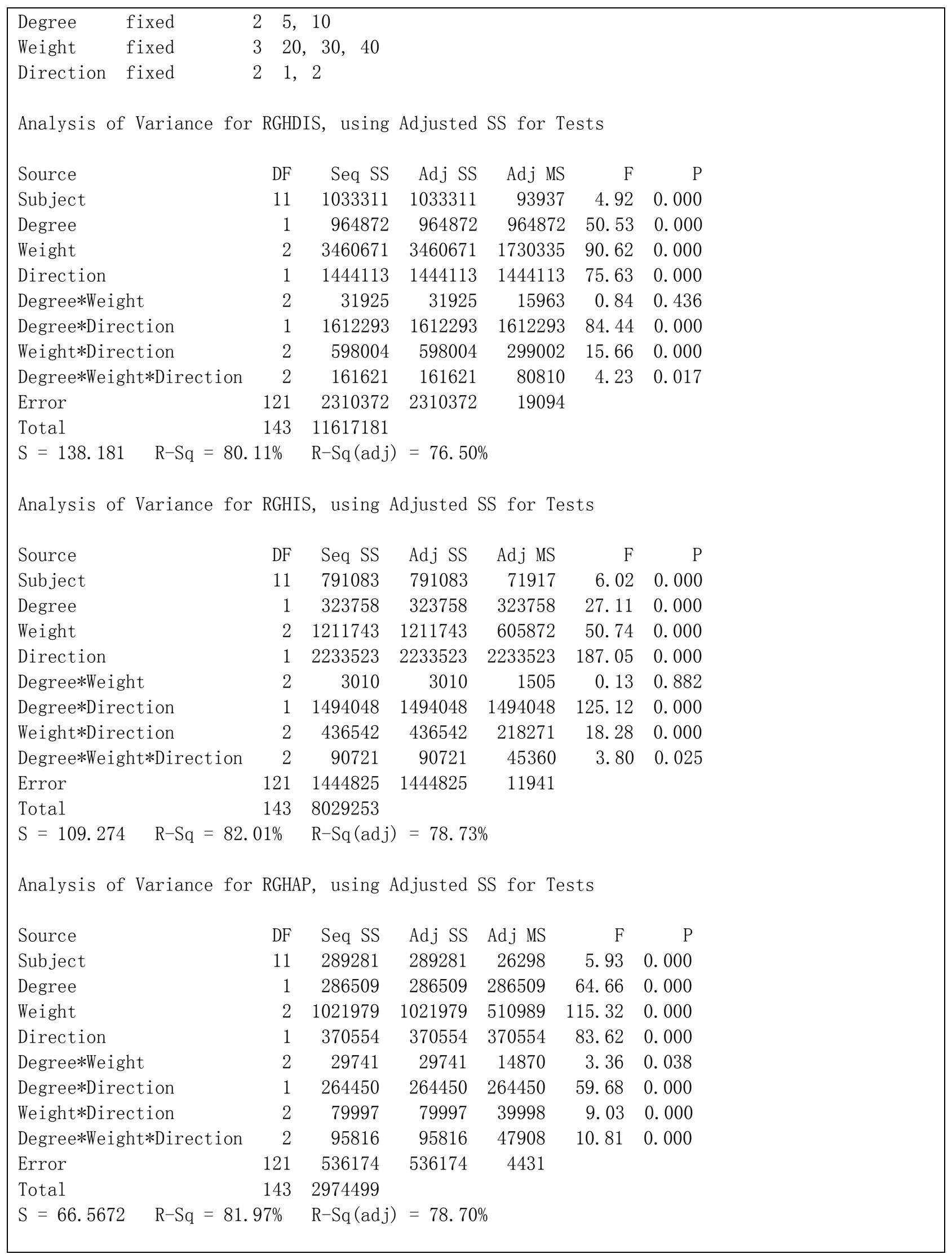


APPENDIX K: ALL-PAIRWISE COMPARISON TEST TABLES FOR MODEL 2 
Table K-1: Tukey HSD All-pairwise comparisons test for averaged peak L5S1-ML forces for different walkway gradient, load weight and direction of force application along ramps

An asterisk $(*)$ represents a significant difference

\begin{tabular}{|c|c|c|c|c|c|c|c|c|c|c|c|c|c|c|}
\hline Degree & Weight & Direction & Mean & $5,20,1$ & $5,20,2$ & $5,30,1$ & $5,30,2$ & $5,40,1$ & $5,40,2$ & $10,20,1$ & $10,20,2$ & $10,30,1$ & $10,30,2$ & $10,40,1$ \\
\hline $5^{\circ}$ & $20 \mathrm{~kg}$ & 1-up & 62.1 & & & & & & & & & & & \\
\hline $5^{\circ}$ & $20 \mathrm{~kg}$ & 2-down & 59.2 & -2.9 & & & & & & & & & & \\
\hline $5^{\circ}$ & $30 \mathrm{~kg}$ & 1-up & 83.3 & 21.2 & $24.1^{*}$ & & & & & & & & & \\
\hline $5^{\circ}$ & $30 \mathrm{~kg}$ & 2-down & 80.8 & 18.7 & 21.6 & -2.5 & & & & & & & & \\
\hline $5^{\circ}$ & $40 \mathrm{~kg}$ & 1-up & 126.0 & $63.9 *$ & $66.8^{*}$ & $42.7 *$ & $45.2 *$ & & & & & & & \\
\hline $5^{\circ}$ & $40 \mathrm{~kg}$ & 2-down & 97.8 & $35.7 *$ & $38.6^{*}$ & 14.5 & 17.0 & $-28.2^{*}$ & & & & & & \\
\hline $10^{\circ}$ & $20 \mathrm{~kg}$ & 1-up & 77.6 & 15.5 & 18.4 & -5.7 & -3.2 & $-48.4 *$ & -20.2 & & & & & \\
\hline $10^{\circ}$ & $20 \mathrm{~kg}$ & 2-down & 55.4 & -6.7 & -3.8 & $-27.9^{*}$ & $-25.4^{*}$ & $-70.6^{*}$ & $-42.4 *$ & -22.2 & & & & \\
\hline $10^{\circ}$ & $30 \mathrm{~kg}$ & 1-up & 87.5 & $25.4^{*}$ & $28.3^{*}$ & 4.2 & 6.7 & $-38.5^{*}$ & -10.3 & 9.9 & $32.1 *$ & & & \\
\hline $10^{\circ}$ & $30 \mathrm{~kg}$ & 2-down & 63.7 & 1.6 & 4.5 & -19.6 & -17.1 & $-62.3 *$ & $-34.1 *$ & -13.9 & 8.3 & $-23.8^{*}$ & & \\
\hline $10^{\circ}$ & $40 \mathrm{~kg}$ & 1-up & 139.3 & $77.2^{*}$ & $80.1^{*}$ & $56.0 *$ & $58.5^{*}$ & 13.3 & $41.5^{*}$ & $61.7 *$ & $83.9 *$ & $51.8^{*}$ & $75.6^{*}$ & \\
\hline $10^{\circ}$ & $40 \mathrm{~kg}$ & 2-down & 74.6 & 12.5 & 15.4 & -8.7 & -6.2 & $-51.4^{*}$ & -23.2 & -3.0 & 19.2 & -12.9 & 10.9 & $-64.7 *$ \\
\hline
\end{tabular}

Table K-2: Tukey HSD All-pairwise comparisons test for averaged peak L5S1-PD forces for different walkway gradient, load weight and direction of force application along ramps

\begin{tabular}{|c|c|c|c|c|c|c|c|c|c|c|c|c|c|c|}
\hline Degree & Weight & Direction & Mean & $5,20,1$ & $5,20,2$ & $5,30,1$ & $5,30,2$ & $5,40,1$ & $5,40,2$ & $10,20,1$ & $10,20,2$ & $10,30,1$ & $10,30,2$ & $10,40,1$ \\
\hline $5^{\circ}$ & $20 \mathrm{~kg}$ & 1-up & 1159.6 & & & & & & & & & & & \\
\hline $5^{\circ}$ & $20 \mathrm{~kg}$ & 2-down & 955.8 & -204 & & & & & & & & & & \\
\hline $5^{\circ}$ & $30 \mathrm{~kg}$ & 1-up & 1498.6 & 339 & $543 *$ & & & & & & & & & \\
\hline $5^{\circ}$ & $30 \mathrm{~kg}$ & 2-down & 1415.7 & 256 & 460 & -83 & & & & & & & & \\
\hline $5^{\circ}$ & $40 \mathrm{~kg}$ & 1-up & 1898.5 & $739 *$ & $943 *$ & 400 & 483 & & & & & & & \\
\hline $5^{\circ}$ & $40 \mathrm{~kg}$ & 2-down & 2203.8 & $1044^{*}$ & $1248^{*}$ & $705^{*}$ & $788^{*}$ & 305 & & & & & & \\
\hline $10^{\circ}$ & $20 \mathrm{~kg}$ & 1-up & 1468.6 & 309 & $513 *$ & -30 & 53 & -430 & $-735 *$ & & & & & \\
\hline $10^{\circ}$ & $20 \mathrm{~kg}$ & 2-down & 1018.8 & -141 & 63 & -480 & -397 & $-879 *$ & $-1185^{*}$ & -450 & & & & \\
\hline $10^{\circ}$ & $30 \mathrm{~kg}$ & 1-up & 2420.0 & $1260 *$ & $1464 *$ & $921 *$ & $1004 *$ & $521.5 *$ & 216 & $951 *$ & $1401 *$ & & & \\
\hline $10^{\circ}$ & $30 \mathrm{~kg}$ & 2-down & 1026.8 & -133 & 71 & -472 & -389 & $-871 *$ & $-1177 *$ & -442 & 8 & $-1393 *$ & & \\
\hline $10^{\circ}$ & $40 \mathrm{~kg}$ & 1-up & 3222.1 & $2062 *$ & $2266^{*}$ & $1723^{*}$ & 1806* & $1323 *$ & $1018 *$ & $1753^{*}$ & $2203^{*}$ & $802 *$ & $2195^{*}$ & \\
\hline $10^{\circ}$ & $40 \mathrm{~kg}$ & 2-down & 1188.5 & 29 & 233 & -310 & -227 & $-710 *$ & $-1015^{*}$ & -280 & 170 & $-1231 *$ & 162 & $-2033 *$ \\
\hline
\end{tabular}


Table K-3: Tukey HSD All-pairwise comparisons test for averaged peak L5S1-AP forces for different walkway gradient, load weight and direction of force application along ramps

\begin{tabular}{|c|c|c|c|c|c|c|c|c|c|c|c|c|c|c|}
\hline Degree & Weight & Direction & Mean & $5,20,1$ & $5,20,2$ & $5,30,1$ & $5,30,2$ & $5,40,1$ & $5,40,2$ & $10,20,1$ & $10,20,2$ & $10,30,1$ & $10,30,2$ & $10,40,1$ \\
\hline $5^{\circ}$ & $20 \mathrm{~kg}$ & 1-up & 259.6 & & & & & & & & & & & \\
\hline $5^{\circ}$ & $20 \mathrm{~kg}$ & 2-down & 186.5 & -73.1 & & & & & & & & & & \\
\hline $5^{\circ}$ & $30 \mathrm{~kg}$ & 1-up & 343.0 & 83.4 & $156.5 *$ & & & & & & & & & \\
\hline $5^{\circ}$ & $30 \mathrm{~kg}$ & 2-down & 277.9 & 18.3 & 91.4 & -65.1 & & & & & & & & \\
\hline $5^{\circ}$ & $40 \mathrm{~kg}$ & 1-up & 421.9 & $162.3^{*}$ & $235.4 *$ & 78.9 & $144.0 *$ & & & & & & & \\
\hline $5^{\circ}$ & $40 \mathrm{~kg}$ & 2-down & 374.7 & $115.1^{*}$ & $188.2 *$ & 31.7 & 96.8 & -47.2 & & & & & & \\
\hline $10^{\circ}$ & $20 \mathrm{~kg}$ & 1-up & 329.4 & 69.8 & $142.9 *$ & -13.6 & 51.5 & -92.5 & -45.3 & & & & & \\
\hline $10^{\circ}$ & $20 \mathrm{~kg}$ & 2-down & 196.2 & -63.4 & 9.7 & $-146.8 *$ & -81.7 & $-225.7 *$ & $-178.5^{*}$ & $-133.2 *$ & & & & \\
\hline $10^{\circ}$ & $30 \mathrm{~kg}$ & 1-up & 553.2 & $293.6 *$ & $366.7 *$ & $210.2 *$ & $275.3 *$ & $131.3^{*}$ & $178.5^{*}$ & $223.8 *$ & $357.0^{*}$ & & & \\
\hline $10^{\circ}$ & $30 \mathrm{~kg}$ & 2-down & 217.9 & -41.7 & 31.4 & $-125.1 *$ & -60.0 & $-204.0 *$ & $-156.8 *$ & $-111.5^{*}$ & 21.7 & $-335.3^{*}$ & & \\
\hline $10^{\circ}$ & $40 \mathrm{~kg}$ & 1-up & 806.2 & $546.6^{*}$ & 619.7* & $463.2 *$ & $528.3 *$ & $384.3 *$ & $431.5^{*}$ & $476.8 *$ & $610.0 *$ & $253.0 *$ & $588.3 *$ & \\
\hline $10^{\circ}$ & $40 \mathrm{~kg}$ & 2-down & 237.8 & -21.8 & 51.3 & -105.2 & -40.1 & $-184.1 *$ & $-136.9 *$ & -91.6 & 41.6 & $-315.4 *$ & 19.9 & $-568.4^{*}$ \\
\hline
\end{tabular}

Table K-4: Tukey HSD All-pairwise comparisons test for averaged peak RSC-ML forces for different walkway gradient, load weight and direction of force application along ramps

\begin{tabular}{|c|c|c|c|c|c|c|c|c|c|c|c|c|c|c|}
\hline Degree & Weight & Direction & Mean & $5,20,1$ & $5,20,2$ & $5,30,1$ & $5,30,2$ & $5,40,1$ & $5,40,2$ & $10,20,1$ & $10,20,2$ & $10,30,1$ & $10,30,2$ & $10,40,1$ \\
\hline $5^{\circ}$ & $20 \mathrm{~kg}$ & 1-up & 72.4 & & & & & & & & & & & \\
\hline $5^{\circ}$ & $20 \mathrm{~kg}$ & 2-down & 69.7 & -2.7 & & & & & & & & & & \\
\hline $5^{\circ}$ & $30 \mathrm{~kg}$ & 1-up & 78.2 & 5.8 & 8.5 & & & & & & & & & \\
\hline $5^{\circ}$ & $30 \mathrm{~kg}$ & 2-down & 115.2 & $42.8 *$ & $45.5^{*}$ & $37.0 *$ & & & & & & & & \\
\hline $5^{\circ}$ & $40 \mathrm{~kg}$ & 1-up & 81.9 & 9.5 & 12.2 & 3.7 & -33.3 & & & & & & & \\
\hline $5^{\circ}$ & $40 \mathrm{~kg}$ & 2-down & 170.5 & $98.1 *$ & $100.8^{*}$ & $92.3 *$ & $55.3 *$ & $88.6^{*}$ & & & & & & \\
\hline $10^{\circ}$ & $20 \mathrm{~kg}$ & 1-up & 82.6 & 10.2 & 12.9 & 4.4 & -32.6 & 0.7 & $-87.9 *$ & & & & & \\
\hline $10^{\circ}$ & $20 \mathrm{~kg}$ & 2-down & 64.5 & -7.9 & -5.2 & -13.7 & $-50.7 *$ & -17.4 & $-106 *$ & -18.1 & & & & \\
\hline $10^{\circ}$ & $30 \mathrm{~kg}$ & 1-up & 190.9 & $118.5^{*}$ & $121.2 *$ & $112.7 *$ & $75.7 *$ & $109.0 *$ & 20.4 & $108.3^{*}$ & $126.4 *$ & & & \\
\hline $10^{\circ}$ & $30 \mathrm{~kg}$ & 2-down & 78.7 & 6.3 & 9.0 & 0.5 & $-36.5 *$ & -3.2 & $-91.8 *$ & -3.9 & 14.2 & $-112.2 *$ & & \\
\hline $10^{\circ}$ & $40 \mathrm{~kg}$ & 1-up & 210.6 & $138.2 *$ & $140.9 *$ & $132.4 *$ & $95.4 *$ & $128.7 *$ & $40.1^{*}$ & $128.0 *$ & $146.1^{*}$ & 19.7 & $131.9 *$ & \\
\hline $10^{\circ}$ & $40 \mathrm{~kg}$ & 2-down & 71.2 & -1.2 & 1.5 & -7.0 & $-44.0 *$ & -10.7 & $-99.3 *$ & -11.4 & 6.7 & $-119.7 *$ & -7.5 & $-139.4^{*}$ \\
\hline
\end{tabular}


Table K-5: Tukey HSD All-pairwise comparisons test for averaged peak RSC-IS forces for different walkway gradient, load weight and direction of force application along ramps

\begin{tabular}{|c|c|c|c|c|c|c|c|c|c|c|c|c|c|c|}
\hline Degree & Weight & Direction & Mean & $5,20,1$ & $5,20,2$ & $5,30,1$ & $5,30,2$ & $5,40,1$ & $5,40,2$ & $10,20,1$ & $10,20,2$ & $10,30,1$ & $10,30,2$ & $10,40,1$ \\
\hline $5^{\circ}$ & $20 \mathrm{~kg}$ & 1-up & 70.3 & & & & & & & & & & & \\
\hline $5^{\circ}$ & $20 \mathrm{~kg}$ & 2-down & 81.8 & 11.5 & & & & & & & & & & \\
\hline $5^{\circ}$ & $30 \mathrm{~kg}$ & 1-up & 85.6 & 15.3 & 3.8 & & & & & & & & & \\
\hline $5^{\circ}$ & $30 \mathrm{~kg}$ & 2-down & 89.5 & 19.2 & 7.7 & 3.9 & & & & & & & & \\
\hline $5^{\circ}$ & $40 \mathrm{~kg}$ & 1-up & 138.8 & $68.5 *$ & $57.0 *$ & $53.2 *$ & $49.3 *$ & & & & & & & \\
\hline $5^{\circ}$ & $40 \mathrm{~kg}$ & 2-down & 132.7 & $62.4 *$ & $50.9 *$ & $47.1 *$ & $43.2 *$ & -6.1 & & & & & & \\
\hline $10^{\circ}$ & $20 \mathrm{~kg}$ & 1-up & 100.6 & $30.3 *$ & 18.8 & 15.0 & 11.1 & $-38.2^{*}$ & $-32.1 *$ & & & & & \\
\hline $10^{\circ}$ & $20 \mathrm{~kg}$ & 2-down & 75.3 & 5.0 & -6.5 & -10.3 & -14.2 & $-63.5^{*}$ & $-57.4^{*}$ & -25.3 & & & & \\
\hline $10^{\circ}$ & $30 \mathrm{~kg}$ & 1-up & 145.5 & $75.2 *$ & $63.7 *$ & $59.9 *$ & $56.0 *$ & 6.7 & 12.8 & $44.9 *$ & $70.2 *$ & & & \\
\hline $10^{\circ}$ & $30 \mathrm{~kg}$ & 2-down & 93.0 & 22.7 & 11.2 & 7.4 & 3.5 & $-45.8 *$ & $-39.7^{*}$ & -7.6 & 17.7 & $-52.5 *$ & & \\
\hline $10^{\circ}$ & $40 \mathrm{~kg}$ & 1-up & 200.3 & $130 *$ & $118.5^{*}$ & $114.7 *$ & $110.8 *$ & $61.5 *$ & $67.6^{*}$ & $99.7 *$ & $125.0 *$ & $54.8 *$ & $107.3 *$ & \\
\hline $10^{\circ}$ & $40 \mathrm{~kg}$ & 2-down & 87.9 & 17.6 & 6.1 & 2.3 & -1.6 & $-50.9 *$ & $-44.8 *$ & -12.7 & 12.6 & $-57.6^{*}$ & -5.1 & $-112.4^{*}$ \\
\hline
\end{tabular}

Table K-6: Tukey HSD All-pairwise comparisons test for averaged peak RSC-AP forces for different walkway gradient, load weight and direction of force application along ramps

\begin{tabular}{|c|c|c|c|c|c|c|c|c|c|c|c|c|c|c|}
\hline Degree & Weight & Direction & Mean & $5,20,1$ & $5,20,2$ & $5,30,1$ & $5,30,2$ & $5,40,1$ & $5,40,2$ & $10,20,1$ & $10,20,2$ & $10,30,1$ & $10,30,2$ & $10,40,1$ \\
\hline $5^{\circ}$ & $20 \mathrm{~kg}$ & 1-up & 26.6 & & & & & & & & & & & \\
\hline $5^{\circ}$ & $20 \mathrm{~kg}$ & 2-down & 29.4 & 2.8 & & & & & & & & & & \\
\hline $5^{\circ}$ & $30 \mathrm{~kg}$ & 1-up & 37.7 & $11.1 *$ & 8.3 & & & & & & & & & \\
\hline $5^{\circ}$ & $30 \mathrm{~kg}$ & 2-down & 38.9 & $12.3 *$ & $9.5 *$ & 1.2 & & & & & & & & \\
\hline $5^{\circ}$ & $40 \mathrm{~kg}$ & 1-up & 42.4 & $15.8 *$ & $13.0 *$ & 4.7 & 3.5 & & & & & & & \\
\hline $5^{\circ}$ & $40 \mathrm{~kg}$ & 2-down & 60.5 & $33.9 *$ & $31.1 *$ & $22.8 *$ & $21.6^{*}$ & $18.1^{*}$ & & & & & & \\
\hline $10^{\circ}$ & $20 \mathrm{~kg}$ & 1-up & 47.5 & $20.9 *$ & $18.1^{*}$ & $9.8 *$ & $8.6^{*}$ & 5.1 & $-13.0 *$ & & & & & \\
\hline $10^{\circ}$ & $20 \mathrm{~kg}$ & 2-down & 29.3 & 2.7 & -0.1 & -8.4 & $-9.6^{*}$ & $-13.1 *$ & $-31.2 *$ & $-18.2 *$ & & & & \\
\hline $10^{\circ}$ & $30 \mathrm{~kg}$ & 1-up & 47.5 & $20.9 *$ & $18.1 *$ & $9.8 *$ & $8.6^{*}$ & 5.1 & $-13.0 *$ & 0.0 & $18.2 *$ & & & \\
\hline $10^{\circ}$ & $30 \mathrm{~kg}$ & 2-down & 37.9 & $11.3 *$ & $8.5 *$ & 0.2 & -1.0 & -4.5 & $-22.6^{*}$ & $-9.6^{*}$ & $8.6^{*}$ & $-9.6 *$ & & \\
\hline $10^{\circ}$ & $40 \mathrm{~kg}$ & 1-up & 60.6 & $34.0 *$ & $31.2 *$ & $22.9 *$ & $21.7 *$ & $18.2 *$ & 0.1 & $13.1 *$ & $31.3 *$ & $13.1 *$ & $22.7 *$ & \\
\hline $10^{\circ}$ & $40 \mathrm{~kg}$ & 2-down & 44.8 & $18.2 *$ & $15.4 *$ & 7.1 & 5.9 & 2.4 & $-15.7 *$ & -2.7 & $15.5^{*}$ & -2.7 & 6.9 & $-15.8 *$ \\
\hline
\end{tabular}


Table K-7: Tukey HSD All-pairwise comparisons test for averaged peak RAC-ML forces for different walkway gradient, load weight and direction of force application along ramps

\begin{tabular}{|c|c|c|c|c|c|c|c|c|c|c|c|c|c|c|}
\hline Degree & Weight & Direction & Mean & $5,20,1$ & $5,20,2$ & $5,30,1$ & $5,30,2$ & $5,40,1$ & $5,40,2$ & $10,20,1$ & $10,20,2$ & $10,30,1$ & $10,30,2$ & $10,40,1$ \\
\hline $5^{\circ}$ & $20 \mathrm{~kg}$ & 1-up & 235.9 & & & & & & & & & & & \\
\hline $5^{\circ}$ & $20 \mathrm{~kg}$ & 2-down & 265.3 & 29.4 & & & & & & & & & & \\
\hline $5^{\circ}$ & $30 \mathrm{~kg}$ & 1-up & 335.8 & 99.9 & 70.5 & & & & & & & & & \\
\hline $5^{\circ}$ & $30 \mathrm{~kg}$ & 2-down & 380.9 & $145.0^{*}$ & 115.6 & 45.1 & & & & & & & & \\
\hline $5^{\circ}$ & $40 \mathrm{~kg}$ & 1-up & 453.5 & $217.6^{*}$ & $188.2 *$ & 117.7 & 72.6 & & & & & & & \\
\hline $5^{\circ}$ & $40 \mathrm{~kg}$ & 2-down & 470.9 & $235.0^{*}$ & $205.6^{*}$ & 135.1 & 90.0 & 17.4 & & & & & & \\
\hline $10^{\circ}$ & $20 \mathrm{~kg}$ & 1-up & 401.0 & $165.1^{*}$ & 135.7 & 65.2 & 20.1 & -52.5 & -69.9 & & & & & \\
\hline $10^{\circ}$ & $20 \mathrm{~kg}$ & 2-down & 278.2 & 42.3 & 12.9 & -57.6 & -102.7 & $-175^{*}$ & $-193^{*}$ & -122.8 & & & & \\
\hline $10^{\circ}$ & $30 \mathrm{~kg}$ & 1-up & 557.6 & $321.7 *$ & $292.3^{*}$ & $221.8^{*}$ & $176.7 *$ & 104.1 & 86.7 & $156.6^{*}$ & $279.4 *$ & & & \\
\hline $10^{\circ}$ & $30 \mathrm{~kg}$ & 2-down & 350.2 & 114.3 & 84.9 & 14.4 & -30.7 & -103.3 & -120.7 & -50.8 & 72.0 & $-207.4 *$ & & \\
\hline $10^{\circ}$ & $40 \mathrm{~kg}$ & 1-up & 910.0 & $674.1^{*}$ & $644.7 *$ & $574.2^{*}$ & $529.1^{*}$ & $456.5^{*}$ & $439.1^{*}$ & $509.0^{*}$ & $631.8^{*}$ & $352.4^{*}$ & $559.8 *$ & \\
\hline $10^{\circ}$ & $40 \mathrm{~kg}$ & 2-down & 366.1 & 130.2 & 100.8 & 30.3 & -14.8 & -87.4 & -104.8 & -34.9 & 87.9 & $-191.5^{*}$ & 15.9 & $-543.9^{*}$ \\
\hline
\end{tabular}

Table K-8: Tukey HSD All-pairwise comparisons test for averaged peak RAC-IS forces for different walkway gradient, load weight and direction of force application along ramps

\begin{tabular}{|c|c|c|c|c|c|c|c|c|c|c|c|c|c|c|}
\hline Degree & Weight & Direction & Mean & $5,20,1$ & $5,20,2$ & $5,30,1$ & $5,30,2$ & $5,40,1$ & $5,40,2$ & $10,20,1$ & $10,20,2$ & $10,30,1$ & $10,30,2$ & $10,40,1$ \\
\hline $5^{\circ}$ & $20 \mathrm{~kg}$ & 1-up & 298.4 & & & & & & & & & & & \\
\hline $5^{\circ}$ & $30 \mathrm{~kg}$ & 1-up & 420.0 & 121.6 & 147.1 & & & & & & & & & \\
\hline $5^{\circ}$ & $40 \mathrm{~kg}$ & 1-up & 625.6 & $327.2^{*}$ & $352.7 *$ & $205.6^{*}$ & $203.5^{*}$ & & & & & & & \\
\hline $5^{\circ}$ & $40 \mathrm{~kg}$ & 2-down & 565.4 & $267.0^{*}$ & $292.5^{*}$ & 145.4 & 143.3 & -60.2 & & & & & & \\
\hline $10^{\circ}$ & $20 \mathrm{~kg}$ & 1-up & 515.3 & $216.9^{*}$ & $242.4^{*}$ & 95.3 & 93.2 & -110.3 & -50.1 & & & & & \\
\hline $10^{\circ}$ & $30 \mathrm{~kg}$ & 2-down & 329.6 & 31.2 & 56.7 & -90.4 & -92.5 & $-296.0^{*}$ & $-235.8^{*}$ & $-185.7 *$ & 37.7 & $-453.9^{*}$ & & \\
\hline $10^{\circ}$ & $40 \mathrm{~kg}$ & 1-up & 1078 & 779.7* & $805.2^{*}$ & $658.1^{*}$ & $656.0^{*}$ & $452.5^{*}$ & $512.7 *$ & $562.8^{*}$ & $786.2^{*}$ & $294.6^{*}$ & $748.5^{*}$ & \\
\hline $10^{\circ}$ & $40 \mathrm{~kg}$ & 2-down & 373.2 & 74.8 & 100.3 & -46.8 & -48.9 & $-252.4^{*}$ & $-192.2^{*}$ & -142.1 & 81.3 & $-410.3^{*}$ & 43.6 & $-704.9 *$ \\
\hline
\end{tabular}


Table K-9: Tukey HSD All-pairwise comparisons test for averaged peak RAC-AP forces for different walkway gradient, load weight and direction of force application along ramps

\begin{tabular}{|c|c|c|c|c|c|c|c|c|c|c|c|c|c|c|}
\hline Degree & Weight & Direction & Mean & $5,20,1$ & $5,20,2$ & $5,30,1$ & $5,30,2$ & $5,40,1$ & $5,40,2$ & $10,20,1$ & $10,20,2$ & $10,30,1$ & $10,30,2$ & $10,40,1$ \\
\hline $5^{\circ}$ & $20 \mathrm{~kg}$ & 1-up & 202.1 & & & & & & & & & & & \\
\hline $5^{\circ}$ & $20 \mathrm{~kg}$ & 2-down & 191.9 & -10.2 & & & & & & & & & & \\
\hline $5^{\circ}$ & $30 \mathrm{~kg}$ & 1-up & 248.7 & 46.6 & 56.8 & & & & & & & & & \\
\hline $5^{\circ}$ & $30 \mathrm{~kg}$ & 2-down & 278.5 & 76.4 & 86.6 & 29.8 & & & & & & & & \\
\hline $5^{\circ}$ & $40 \mathrm{~kg}$ & 1-up & 360.0 & $157.9 *$ & $168.1^{*}$ & $111.3^{*}$ & 81.5 & & & & & & & \\
\hline $5^{\circ}$ & $40 \mathrm{~kg}$ & 2-down & 337.5 & $135.4^{*}$ & $145.6^{*}$ & 88.8 & 59.0 & -22.5 & & & & & & \\
\hline $10^{\circ}$ & $20 \mathrm{~kg}$ & 1-up & 286.3 & 84.2 & $94.4^{*}$ & 37.6 & 7.8 & -73.7 & -51.2 & & & & & \\
\hline $10^{\circ}$ & $20 \mathrm{~kg}$ & 2-down & 201.5 & -0.6 & 9.6 & -47.2 & -77.0 & $-158.5^{*}$ & $-136.0 *$ & -84.8 & & & & \\
\hline $10^{\circ}$ & $30 \mathrm{~kg}$ & 1-up & 479.0 & $276.9 *$ & $287.1^{*}$ & $230.3^{*}$ & $200.5^{*}$ & $119.0^{*}$ & $141.5^{*}$ & $192.7^{*}$ & $277.5^{*}$ & & & \\
\hline $10^{\circ}$ & $30 \mathrm{~kg}$ & 2-down & 198.8 & -3.3 & 6.9 & -49.9 & -79.7 & $-161.2 *$ & $-138.7 *$ & -87.5 & -2.7 & $-280.2 *$ & & \\
\hline $10^{\circ}$ & $40 \mathrm{~kg}$ & 1-up & 726.8 & $524.7 *$ & $534.9 *$ & $478.1^{*}$ & $448.3^{*}$ & $366.8 *$ & $389.3 *$ & $440.5^{*}$ & $525.3 *$ & $247.8 *$ & $528.0 *$ & \\
\hline $10^{\circ}$ & $40 \mathrm{~kg}$ & 2-down & 226.8 & 24.7 & 34.9 & -21.9 & -51.7 & $-133.2 *$ & $-110.7 *$ & -59.5 & 25.3 & $-252.2 *$ & 28.0 & $-500.0 *$ \\
\hline
\end{tabular}

Table K-10: Tukey HSD All-pairwise comparisons test for averaged peak RGH-DIS forces for different walkway gradient, load weight and direction of force application along ramps

\begin{tabular}{|c|c|c|c|c|c|c|c|c|c|c|c|c|c|c|}
\hline Degree & Weight & Direction & Mean & $5,20,1$ & $5,20,2$ & $5,30,1$ & $5,30,2$ & $5,40,1$ & $5,40,2$ & $10,20,1$ & $10,20,2$ & $10,30,1$ & $10,30,2$ & $10,40,1$ \\
\hline $5^{\circ}$ & $20 \mathrm{~kg}$ & 1-up & 447.4 & & & & & & & & & & & \\
\hline $5^{\circ}$ & $20 \mathrm{~kg}$ & 2-down & 508.1 & 60.7 & & & & & & & & & & \\
\hline $5^{\circ}$ & $30 \mathrm{~kg}$ & 1-up & 650.2 & $202.8^{*}$ & 142.1 & & & & & & & & & \\
\hline $5^{\circ}$ & $30 \mathrm{~kg}$ & 2-down & 712.1 & $264.7 *$ & $204.0 *$ & 61.9 & & & & & & & & \\
\hline $5^{\circ}$ & $40 \mathrm{~kg}$ & 1-up & 868.3 & $420.9 *$ & $360.2 *$ & $218.1 *$ & 156.2 & & & & & & & \\
\hline $5^{\circ}$ & $40 \mathrm{~kg}$ & 2-down & 779.7 & $332.3^{*}$ & $271.6^{*}$ & 129.5 & 67.6 & -88.6 & & & & & & \\
\hline $10^{\circ}$ & $20 \mathrm{~kg}$ & 1-up & 709.2 & $261.8 *$ & $201.1 *$ & 59.0 & -2.9 & -159.1 & -70.5 & & & & & \\
\hline $10^{\circ}$ & $20 \mathrm{~kg}$ & 2-down & 525.7 & 78.3 & 17.6 & -124.5 & -186.4 & $-343 *$ & $-254 *$ & -183.5 & & & & \\
\hline $10^{\circ}$ & $30 \mathrm{~kg}$ & 1-up & 1025.2 & $577.8 *$ & $517.1^{*}$ & $375.0 *$ & $313.1^{*}$ & 156.9 & $245.5^{*}$ & $316.0 *$ & $499.5^{*}$ & & & \\
\hline $10^{\circ}$ & $30 \mathrm{~kg}$ & 2-down & 628.4 & 181.0 & 120.3 & -21.8 & -83.7 & $-240 *$ & -151.3 & -80.8 & 102.7 & $-396.8 *$ & & \\
\hline $10^{\circ}$ & $40 \mathrm{~kg}$ & 1-up & 1357.3 & $909.9 *$ & $849.2 *$ & $707.1 *$ & $645.2 *$ & $489.0 *$ & $577.6^{*}$ & $648.1 *$ & $831.6^{*}$ & $332.1 *$ & $728.9 *$ & \\
\hline $10^{\circ}$ & $40 \mathrm{~kg}$ & 2-down & 702.0 & $254.6^{*}$ & 193.9* & 51.8 & -10.1 & -166.3 & -77.7 & -7.2 & 176.3 & $-323.2 *$ & 73.6 & $-655.3 *$ \\
\hline
\end{tabular}


Table K-11: Tukey HSD All-pairwise comparisons test for averaged peak RGH-IS forces for different walkway gradient, load weight and direction of force application along ramps

\begin{tabular}{|c|c|c|c|c|c|c|c|c|c|c|c|c|c|c|}
\hline Degree & Weight & Direction & Mean & $5,20,1$ & $5,20,2$ & $5,30,1$ & $5,30,2$ & $5,40,1$ & $5,40,2$ & $10,20,1$ & $10,20,2$ & $10,30,1$ & $10,30,2$ & $10,40,1$ \\
\hline $5^{\circ}$ & $30 \mathrm{~kg}$ & 1-up & 329.8 & 110.6 & 117.2 & & & & & & & & & \\
\hline $5^{\circ}$ & $40 \mathrm{~kg}$ & 1-up & 529.6 & $310.4^{*}$ & $317.0^{*}$ & 199.8* & $166.9^{*}$ & & & & & & & \\
\hline $5^{\circ}$ & $40 \mathrm{~kg}$ & 2-down & 367.2 & 148.0 & $154.6^{*}$ & 37.4 & 4.5 & $-162.4 *$ & & & & & & \\
\hline $10^{\circ}$ & $20 \mathrm{~kg}$ & 1-up & 459.3 & $240.1^{*}$ & $246.7 *$ & 129.5 & 96.6 & -70.3 & 92.1 & & & & & \\
\hline $10^{\circ}$ & $20 \mathrm{~kg}$ & 2-down & 187.2 & -32.0 & -25.4 & -142.6 & $-175.5^{*}$ & $-342.4^{*}$ & $-180.0^{*}$ & $-272.1 *$ & & & & \\
\hline $10^{\circ}$ & $30 \mathrm{~kg}$ & 2-down & 207.3 & -11.9 & -5.3 & -122.5 & $-155.4^{*}$ & $-322.3^{*}$ & $-159.9^{*}$ & $-252.0^{*}$ & 20.1 & $-449.3^{*}$ & & \\
\hline $10^{\circ}$ & $40 \mathrm{~kg}$ & 1-up & 858.3 & $639.1^{*}$ & $645.7 *$ & $528.5^{*}$ & $495.6^{*}$ & $328.7 *$ & $491.1 *$ & $399.0 *$ & $671.1^{*}$ & $201.7 *$ & $651.0 *$ & \\
\hline $10^{\circ}$ & $40 \mathrm{~kg}$ & 2-down & 221.3 & 2.1 & 8.7 & -108.5 & -141.4 & $-308.3^{*}$ & -145.9 & $-238.0 *$ & 34.1 & $-435.3^{*}$ & 14.0 & $-637.0^{*}$ \\
\hline
\end{tabular}

Table K-12: Tukey HSD All-pairwise comparisons test for averaged peak RGH-AP forces for different walkway gradient, load weight and direction of force application along ramps

\begin{tabular}{|c|c|c|c|c|c|c|c|c|c|c|c|c|c|c|}
\hline Degree & Weight & Direction & Mean & $5,20,1$ & $5,20,2$ & $5,30,1$ & $5,30,2$ & $5,40,1$ & $5,40,2$ & $10,20,1$ & $10,20,2$ & $10,30,1$ & $10,30,2$ & $10,40,1$ \\
\hline $5^{\circ}$ & $20 \mathrm{~kg}$ & 1-up & 186.4 & & & & & & & & & & & \\
\hline $5^{\circ}$ & $20 \mathrm{~kg}$ & 2-down & 175.3 & -11.1 & & & & & & & & & & \\
\hline $5^{\circ}$ & $30 \mathrm{~kg}$ & 1-up & 277.9 & $91.5^{*}$ & $102.6 *$ & & & & & & & & & \\
\hline $5^{\circ}$ & $30 \mathrm{~kg}$ & 2-down & 233.5 & 47.1 & 58.2 & -44.4 & & & & & & & & \\
\hline $5^{\circ}$ & $40 \mathrm{~kg}$ & 1-up & 348.6 & $162.2^{*}$ & $173.3^{*}$ & 70.7 & $115.1 *$ & & & & & & & \\
\hline $5^{\circ}$ & $40 \mathrm{~kg}$ & 2-down & 356.9 & $170.5^{*}$ & $181.6^{*}$ & 79.0 & $123.4^{*}$ & 8.3 & & & & & & \\
\hline $10^{\circ}$ & $20 \mathrm{~kg}$ & 1-up & 261.1 & 74.7 & 85.8 & -16.8 & 27.6 & -87.5 & $-95.8 *$ & & & & & \\
\hline $10^{\circ}$ & $20 \mathrm{~kg}$ & 2-down & 201.5 & 15.1 & 26.2 & -76.4 & -32.0 & $-147.1 *$ & $-155.4 *$ & -59.6 & & & & \\
\hline $10^{\circ}$ & $30 \mathrm{~kg}$ & 1-up & 458.3 & $271.9 *$ & $283.0 *$ & $180.4 *$ & $224.8 *$ & $109.7 *$ & $101.4^{*}$ & $197.2 *$ & $256.8 *$ & & & \\
\hline $10^{\circ}$ & $30 \mathrm{~kg}$ & 2-down & 249.0 & 62.6 & 73.7 & -28.9 & 15.5 & $-99.6^{*}$ & $-107.9 *$ & -12.1 & 47.5 & $-209.3 *$ & & \\
\hline $10^{\circ}$ & $40 \mathrm{~kg}$ & 1-up & 618.2 & $431.8 *$ & $442.9 *$ & $340.3^{*}$ & $384.7 *$ & $269.6^{*}$ & $261.3 *$ & $357.1 *$ & $416.7 *$ & $159.9 *$ & $369.2 *$ & \\
\hline $10^{\circ}$ & $40 \mathrm{~kg}$ & 2-down & 325.7 & $139.3 *$ & $150.4 *$ & 47.8 & $92.2 *$ & -22.9 & -31.2 & 64.6 & $124.2 *$ & $-132.6 *$ & 76.7 & $-292.5^{*}$ \\
\hline
\end{tabular}


APPENDIX L: REGRESSION ANALYSIS FOR PROBLEMATIC LOADING 
Table L-1: Regression analysis for peak reaction forces acting at the L5S1 joint in anteriorposterior (AP) directions during uphill pushing depending on walkway gradient and load weight

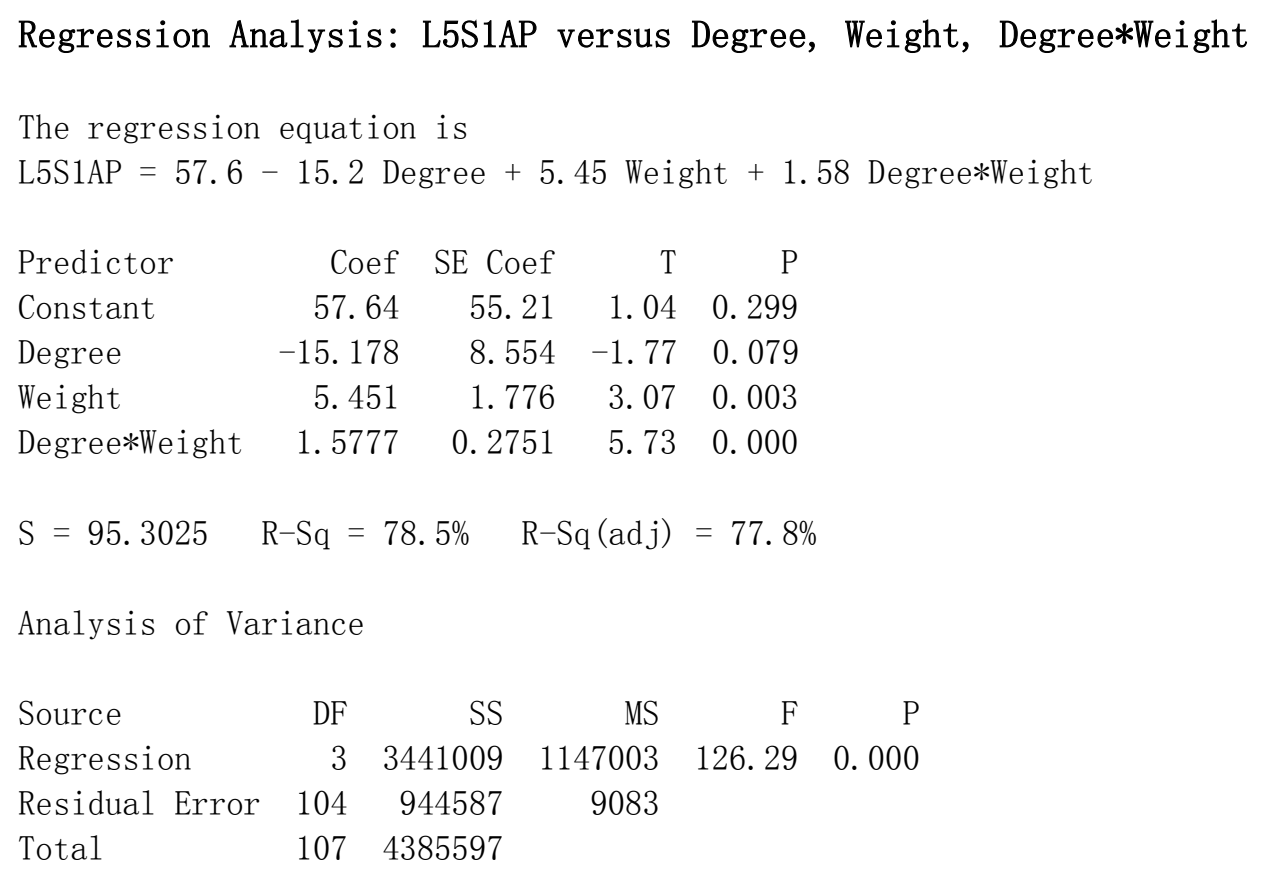

Table L-2: Regression analysis for peak reaction forces acting at the acromioclavicular (AC) joint in inferior-superior (IS) direction during uphill pushing depending on walkway gradient and load weight

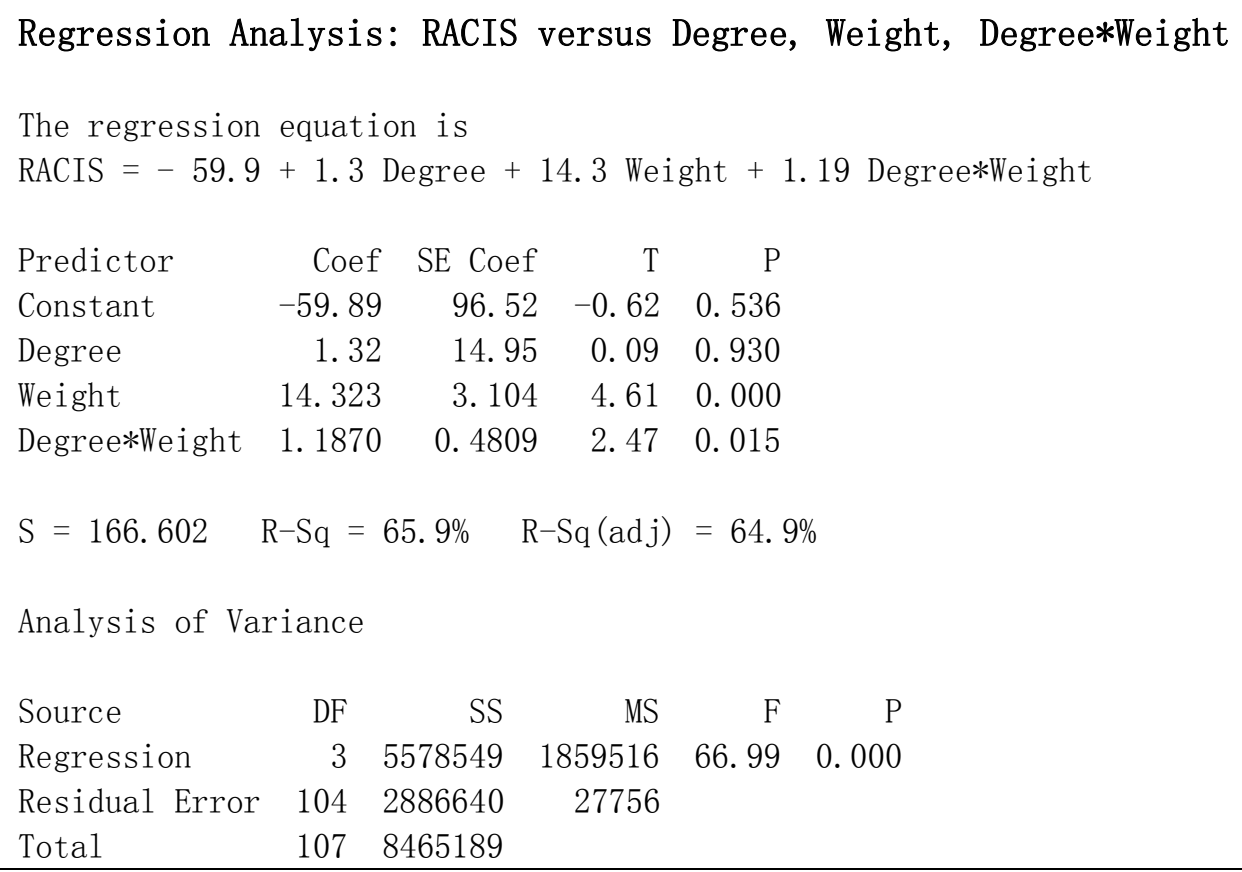


Table L-3: Regression analysis for peak reaction forces acting at the glenohumeral joint (GH) in distraction (DIS) direction during uphill pushing depending on walkway gradient and load weight

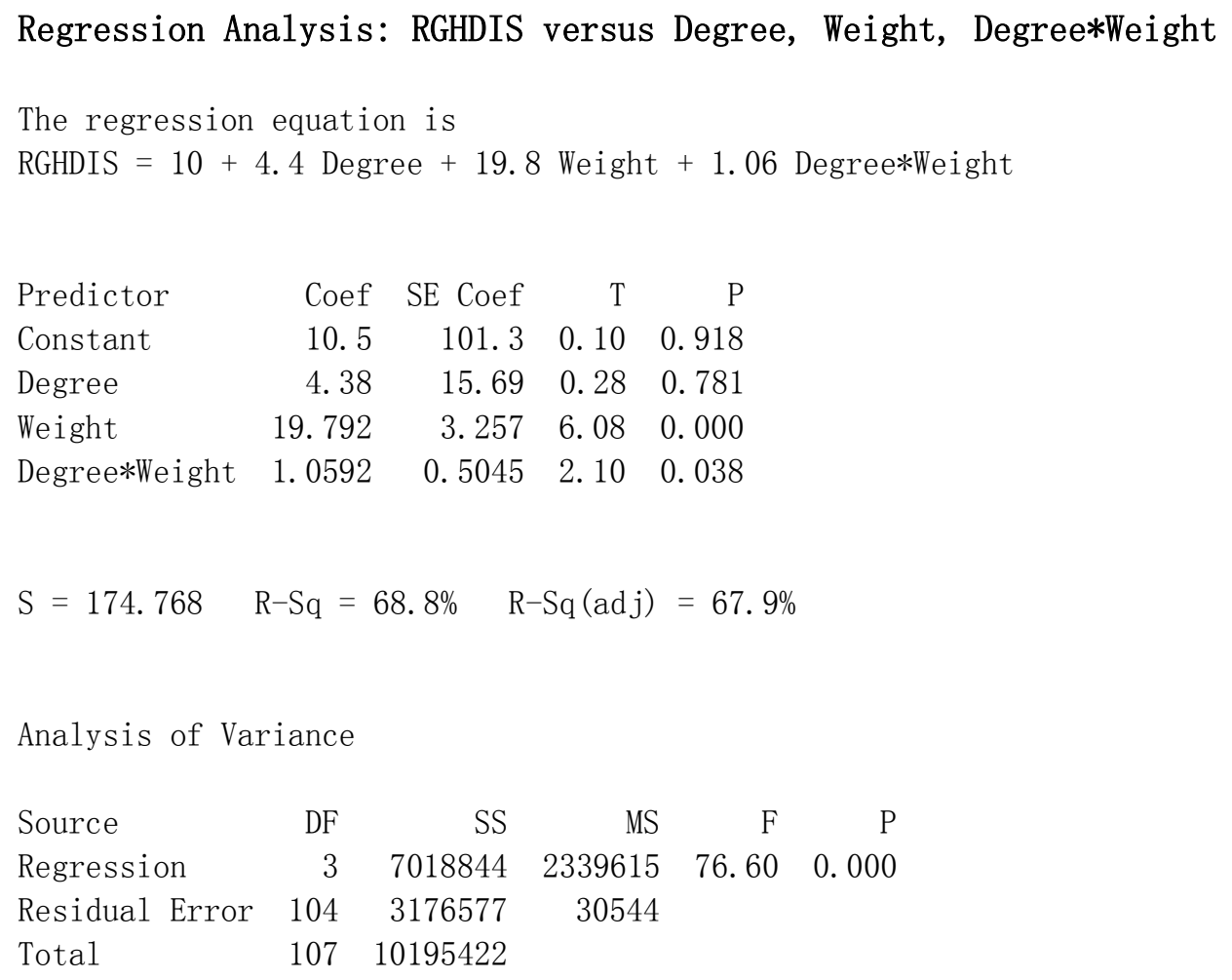

